\title{
Calcineurin-based immunosuppression in renal transplantation : focus on efficacy, the role of steroids, and the cardiovascular risk
}

Citation for published version (APA):

Boots, J. M. M. (2006). Calcineurin-based immunosuppression in renal transplantation : focus on efficacy, the role of steroids, and the cardiovascular risk. [Doctoral Thesis, Maastricht University]. Datawyse / Universitaire Pers Maastricht. https://doi.org/10.26481/dis.20060929jb

Document status and date:

Published: 01/01/2006

DOI:

10.26481/dis.20060929jb

Document Version:

Publisher's PDF, also known as Version of record

Please check the document version of this publication:

- A submitted manuscript is the version of the article upon submission and before peer-review. There can be important differences between the submitted version and the official published version of record.

People interested in the research are advised to contact the author for the final version of the publication, or visit the DOI to the publisher's website.

- The final author version and the galley proof are versions of the publication after peer review.

- The final published version features the final layout of the paper including the volume, issue and page numbers.

Link to publication

\footnotetext{
General rights rights.

- You may freely distribute the URL identifying the publication in the public portal. please follow below link for the End User Agreement:

www.umlib.nl/taverne-license

Take down policy

If you believe that this document breaches copyright please contact us at:

repository@maastrichtuniversity.nl

providing details and we will investigate your claim.
}

Copyright and moral rights for the publications made accessible in the public portal are retained by the authors and/or other copyright owners and it is a condition of accessing publications that users recognise and abide by the legal requirements associated with these

- Users may download and print one copy of any publication from the public portal for the purpose of private study or research.

- You may not further distribute the material or use it for any profit-making activity or commercial gain

If the publication is distributed under the terms of Article $25 \mathrm{fa}$ of the Dutch Copyright Act, indicated by the "Taverne" license above, 


\section{Calcineurin-based immunosuppression in}

renal transplantation: Focus on efficacy, the role of steroids, and the cardiovascular risk 
(C) Johannes Martinus Maria Boots

ISBN-10 90-5278-553-8

ISBN-13 97890-5278-553-0

Cover design: Arie van Vliet Illustraties, Tiel

Layout: Tiny Wouters

Production: Datawyse | Universitaire Pers Maastricht

Deze uitgifte werd financieel ondersteund door de volgende sponsoren, waarvoor natuurlijk hartelijke dank.

Astellas, Baxter, Abbott, Genzyme, Novartis, Amgen, Bristol-Myers-Squibb, Gambro-Hospal, Wyeth, Boeringer-Ingelheim, Pfizer. 


\title{
Calcineurin-based immunosuppression in renal transplantation: Focus on efficacy, the role of steroids, and the cardiovascular risk.
}

\author{
PROEFSCHRIFT \\ Ter verkrijging van de graad van doctor \\ aan de Universiteit Maastricht, \\ op gezag van de Rector Magnificus, Prof. mr. G.P.M.F. Mols, \\ volgens het besluit van het College van Decanen, \\ in het openbaar te verdedigen \\ op vrijdag 29 september 2006 om 14.00 uur \\ door \\ Johannes Martinus Maria Boots
}

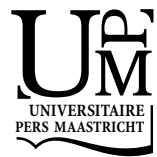


Promotor:

Prof. dr. J.P. van Hooff

\section{Copromotor:}

Dr. M.H.L. Christiaans

Beoordelingscommissie:

Prof. dr. M.J.A.P. Daemen (voorzitter)

Prof. dr. J. Floege, RWTH, Aachen

Dr. T. Goto, Astellas Pharma Inc. Ibaraki, Japan

Prof. dr. P.J.E.H.M. Kitslaar

Prof. dr. C.D.A. Stehouwer 
Voor Sabine 


\section{Contents}

Chapter 1 General introduction 9

$\begin{array}{lll}\text { Chapter } 2 & \text { Single center experience with tacrolimus versus } & 37\end{array}$

cyclosporin-Neoral in renal transplant recipients

Transplant International 2001;14(6): 370-383

Chapter 3 Suppression of the hypothalamic-pituitary-adrenal axis in renal transplantation with steroid-included triple therapy

Transplantation Proceedings 2002;34(5): 1696-1697

Chapter 4 Increase in tacrolimus trough levels after steroid withdrawal Transplant International 2003;16(10):721-725

Chapter 5 Early steroid withdrawal in renal transplantation with tacrolimus dual therapy: A pilot study Transplantation 2002;74(12):1703-1709

Chapter 6 Glucose metabolism in renal transplant recipients on tacrolimus: the effect of steroid withdrawal and tacrolimus trough level reduction Journal of the American Society of Nephrology 2002;13(1):221-227

Chapter 7 Improved cardiovascular risk profile and renal function in renal transplant patients after randomised conversion from cyclosporin to tacrolimus Journal of the American Society of Nephrology 2003;14(7):1880-1888

Chapter 8 Conversion from cyclosporin to tacrolimus improves quality-of-life indices, renal graft function and cardiovascular risk profile American Journal of Transplantation 2004;4(6):937-945

Chapter 9 Effect of immunosuppressive agents on long-term survival of renal transplant recipients: focus on the cardiovascular risk Drugs 2004;64(18):2047-2073

Chapter 10 General discussion

Abbreviations 



\section{Chapter 1}

\section{General introduction}




\section{Introduction}

For patients suffering from renal failure, renal transplantation offers several advantages compared to dialysis, the most important one being that it improves survival and quality of life. This has long been a matter of discussion since renal transplantation is usually only performed on the best candidates. By the end of the last century, it became clear that, despite a small increase in mortality around the transplantation procedure itself, renal transplant recipients have a better survival as early as one year post-transplantation ${ }^{1,2}$. Survival is even improved in recipients of kidneys from marginal donors ${ }^{3}$. Renal transplant recipients experience a better quality of life than patients on either haemodialysis or peritoneal dialysis ${ }^{4}$. Moreover, the costs of a renal transplantation are significantly lower than those associated with maintaining a patient on dialysis 5 .

\section{Immunosuppression}

Unless a transplant recipient receives an organ from an identical twin, a graft will always be recognized as 'foreign' and, consequently, rejected. To prevent this, graft recipients need to be treated with immunosuppressive agents, at least during the initial post-transplantation period. A delicate balance exists between efficacy (prevention of rejection) and toxicity (infections, malignancies, agent-specific side effects). Several immunosuppressive agents are currently registered for use in renal transplantation. In this thesis, we restrict our focus to the efficacy and side effects of steroids, cyclosporin A (CsA), and tacrolimus (TAC), as these are the cornerstone agents.

\section{Steroids}

Steroids were developed in the 1950s, after the discovery of cortisol in 1949 by Hench et al. ${ }^{6}$. The most commonly used preparations are prednisone, prednisolone (the active metabolite of prednisone), and methylprednisolone. The relative potency of methylprednisolone is $25 \%$ higher than that of prednisone or prednisolone ${ }^{7}$. Steroids are used as initial therapy at induction just before surgery, as maintenance therapy, and in high doses as anti-rejection treatment (usually methylprednisolone). The principal target is the intracellular glucocorticoid receptor, which regulates the transcription of many genes and interferes with the stability of certain pro-inflammatory messenger RNAs ${ }^{8}$. The glucocorticoid receptor has two isoforms: type $\alpha$ binds steroids and is responsible for the antiinflammatory effects and type $\beta$, which differs in the carboxy terminus, inhibits the effects of the steroid-activated type $\alpha^{9}$. Steroids have various effects on the immune system, but these are not very specific. Steroids suppress the number of 
circulating lymphocytes by influencing the survival of T-cells and by promoting T-cell apoptosis ${ }^{10}$. They reduce the number of antigen-presenting dendritic cells, the expression of adhesion molecules like intracellular adhesion molecule-1 (ICAM-1), endothelial leukocyte adhesion molecule-1 (ELAM-1), and Eselektine ${ }^{11}$, and the permeability of the capillaries and several chemokines necessary for a proper chemotaxis (prevention of inflammatory cells reaching the tissues to be targeted). They increase the synthesis of several anti-inflammatory proteins, such as lipocortin-1 and interleukin (IL)-1 receptor antagonist. In addition, steroids reduce the level of inflammatory mediators by preventing induction of nitric oxide synthase (NOS) ${ }^{12,13}$ and cyclo-oxygenase-2 (COX-2), and they reduce the number of mast cells. They interfere with the production of a number of T-cell and macrophage-derived cytokines, including IL-1 to IL-6, IL-11 to IL-13, tumour necrosis factor-alpha (TNF- $\alpha$ ), granulocyte macrophage-colonystimulating factor (GM-CSF), and stem cell factor ${ }^{8}$. Steroids also enhance the production of transforming growth factor- $\beta 1$ (TGF- $\beta 1$ ) in human T-cells ${ }^{14}$. TGF$\beta 1$ is a potent inhibitor of the proliferation of T-cells.

Steroids are metabolized by the cytochrome P450 system in the liver, subtype 3A4 (CYP3A4). They appear to be inducers of this enzyme system ${ }^{15-17}$, although less potent than rifampicin ${ }^{16}$, which is commonly known to be a potent inducer. The induction of CYP3A4 may lead to interactions with other drugs metabolized by this enzyme system. Also, steroid exposure increases with the concomitant use of inhibitors of CYP3A4 ${ }^{18,19}$.

Steroids interfere with physiologic steroid production and suppress the hypothalamic-pituitary-adrenal (HPA) axis. Suppression of this HPA axis occurs as early as after one to four weeks of highly dosed steroids ${ }^{20}$. Based on crosssectional data, it has been concluded that the suppressive effect of a given steroid depends on how high the dosage is ${ }^{21-23}$, the duration of the therapy ${ }^{23}$, and the time of administration ${ }^{24}$. Abrupt cessation of steroids, especially in conditions of stress, may result in life-threatening adrenal insufficiency. This dramatic clinical syndrome was first presented in the 1950s by Fraser et al. ${ }^{25}$. Since the second case was reported by Lewis et al. ${ }^{26}$, stress doses of steroids have been recommended for stressful episodes, such as surgery. Discontinuation of steroids should be performed gradually.

Steroids have serious side effects. Aside from those on the cardiovascular system (hypertension, hyperlipidemia, diabetes mellitus), they induce osteoporosis and avascular bone necrosis, gastrointestinal disorders, psychiatric disorders, obesity, cataract, glaucoma, myopathy, tendon ruptures, acne, and disturbance of collagen synthesis, with subsequent atrophy of the skin and vessel wall27-29. 


\section{Cyclosporin A}

CsA is a lipophilic cyclic undecapeptide isolated from the fungus Hypocladium inflatum gams. Its immunosuppressive property was first described in 1976 by Borel et $a .^{30}$. In plasma, CsA dissolves mainly in the lipophilic portion of lipoprotein particles $^{31}$. It has been postulated that the cellular uptake of CsA is mediated by the low-density lipoprotein (LDL) receptor ${ }^{32}$. In 1995, CsA (Sandimmune) was replaced by a micro-emulsion formulation (Neoral) that had a better and more consistent bioavailability ${ }^{33}$.

Remarkably enough, CsA was already being used extensively before its mechanism of action was unravelled. CsA binds to a cytoplasmic receptor, cyclophilin, and this complex blocks the phosphatase activity of calcineurin ${ }^{34}$. Calcineurin consists of two sub-units, a catalytic sub-unit A and a regulatory sub-unit B. After T-cell activation by the T-cell receptor, intracellular calcium is raised, with subsequent activation of calmodulin. Activated calmodulin splices the auto-inhibitory domain from subunit A and activates calcineurin. This activation of calcineurin is blocked by the CsA-cyclophilin complex. This blocking is dependent upon the blood concentration, but CsA blocks calcineurin activity only partially ${ }^{35}$. Calcineurin plays a prominent role in the activation of $\mathrm{T}$-cells ${ }^{36,37}$. It activates them by dephosphorylation of a group of so-called cytosolic nuclear factors of activated T-cells (NF-AT), which are involved in the regulation of gene transcription after moving to the nucleus. NF-AT types 1,2 , and 4 are involved in the transcription of many cytokines, the most important one being IL-2, but also including IL-3, IL-4, TNF- $\alpha$, interferon- $\gamma$, GM-CSF, and the ligand of CD 40 38,39. IL-2 plays a pivotal role in the activation of T-cells. Inhibition of calcineurin also antagonizes the interaction of another transcription factor, cyclic adenosine-monophosphate (c-AMP) response element-binding protein (CREB), with its DNA-binding site CRE, inhibiting c-AMP-directed transcriptional events ${ }^{40,41}$. In addition to the calcineurin/NF-AT pathway, the CsA-cyclophilin complex has recently been recognized as blocking the calcineurin-independent jun $\mathrm{N}$-terminal kinase (JNK) and p38 signaling pathways in the activation of T-cells ${ }^{42,43}$. JNK and p38 are subgroups of protein kinases belonging to a super-family of mitogen-activated protein kinases (MAPK). A cascade of MAPK kinases is activated after activation of the T-cell receptor in the presence of the CD28 co-stimulatory receptor. Cooperative activation of JNK and p38 in conjunction with ERK, a third subgroup of MAPK, leads to activation of transcription factors like activatorprotein-1 (AP-1) ${ }^{38}$. Another mechanism among the immunosuppressive properties of CsA is mediated by its augmentation of the production of TGF- $\beta^{44-46}$. CsA also lowers the production of the inflammation mediator nitric oxide $(\mathrm{NO})^{13,47}$.

Irrespective of the immunosuppressive effects, because non-immunosuppressive derivatives of CsA show similar results, chronic, but not incidental, use of CsA in therapeutic concentrations induces expression of P-glycoprotein (P-gp) ${ }^{48,49}$. P-gp 
is a trans-membrane protein that is responsible for the phenotype of multi-drug resistance (MDR). In humans, two subtypes - MDR-1 and MDR-2 - are recognized, of which the first is responsible for the MDR phenotype. CsA is transported outside the cell by $\mathrm{P}-\mathrm{g} \mathrm{p}^{50}$. After induction of $\mathrm{P}$-gp, increased elimination of CsA from the cell may limit the immunosuppressive efficacy of $\mathrm{CsA}^{49,51}$. Indeed, enhanced expression of P-gp has been observed to result in an increased incidence of acute rejection ${ }^{52}$. Incidental administration of CsA just inhibits the function of $\mathrm{P}^{-g \mathrm{p}^{48}}$. This inhibition is important for the disruption of resistance against cytostatic cancer drugs ${ }^{53}$.

The absorption of CsA shows a large intra- and inter-individual variable bioavailability ${ }^{54}$. Because there is a narrow window between efficacy and toxicity, monitoring of blood levels of the drug is essential. CsA is also metabolized by the Cytochrome P-450 system, subtype $3 \mathrm{~A}^{55}$. Differences in this metabolism account for a substantial part of the inter-individual variability observed with CsA ${ }^{56}$. Since steroids are also metabolized by the same enzyme system, interactions may occur. Indeed, it was shown recently that the area under the curve (AUC) of an equal dose of prednisolone was higher in CsA-treated renal transplant recipients than in healthy controls ${ }^{57}$.

An important side effect of CsA is its nephrotoxicity. This consists of two parts: a reversible, dose-dependent renal vasoconstriction ${ }^{58}$ and a chronic tubulointerstitial fibrosis and vasculopathy. The vasoconstriction is mediated by endothelin-1 (ET-1) in the afferent arteriole ${ }^{59,60}$. CsA augments the action of ETconverting enzyme and the production of ET- 1 by endothelial cells mediated by the enhanced production of TGF- $\beta$ 61. ET-1 also plays a role in the development of the vasculopathy ${ }^{62-64}$. In addition to its immunosuppressive properties, TGF- $\beta$ induces fibrosis formation. CsA upregulates not only TGF- $\beta 1$ expression, but also its receptors in rat mesangial cells ${ }^{65}$. The chronic tubulo-interstitial fibrosis induced by CsA is thought to be mediated by TGF- $\beta 44,66,67$ and by inducing apoptosis in renal tubular cells ${ }^{68}$. However, recently, no toxic effect of CsA on tubular cells could be demonstrated in vitro, and the in vivo results observed may be induced by renal vasoconstriction ${ }^{69}$. CsA may accumulate in the kidney ${ }^{35}$, contributing to its nephrotoxicity.

Hypertrichosis and gingival hyperplasia are other side effects. Both may also be mediated by TGF- $\beta^{70,71}$. TGF- $\beta$ may also directly promote the progression of cancer ${ }^{72}$.

\section{Tacrolimus}

Goto et al, described the isolation of TAC in 1984 from the soil bacterium Streptomyces tsukubaensis, named after the place Tsukuba, in Japan, where the 
bacterium was discovered ${ }^{73}$. TAC, formerly also known as FK-506, has a macrolide structure and appears to be about 100 times more powerful on a molecular basis than $\mathrm{CsA}^{73}$.

Instead of cyclophilin, TAC binds to another cytoplasmic receptor: FK506binding protein (FKBP). FKBPs and cyclophilin are collectively termed immunophilins. The predominant FKBP is FKBP-12 ${ }^{74}$. The normal function of FKBP-12 is probably to anchor calcineurin to cellular $\mathrm{Ca}^{2+}$ channels. In this way, calcineurin modulates cellular $\mathrm{Ca}^{2+} \mathrm{flux}^{75}$. In a similar way, FKBP-12 binds to the type 1 receptors of the TGF- $\beta$ family ${ }^{76,77}$. The complex TAC-FKBP blocks calcineurin, in a manner similar to the CsA-cyclophilin complex, and prevents activation of the T-cell via the NF-AT pathway 34,36,37 and the c-AMP-mediated CREB pathway ${ }^{40}$. Also in a manner similar to cyclosporin, the TAC-FKBP complex blocks T-cell activation via the JNK and p38 activation pathway ${ }^{43}$, and TAC induces the production of TGF- $\beta^{78}$. By blocking cytokine production by T-cells, the proliferation of B-cells and the production of antibodies are also inhibited ${ }^{79}$. Antibody production is reduced more by TAC than by CsA ${ }^{80}$. Another immunophilin, FKBP-52, is associated with the glucocorticoid receptor. Usually, the glucocorticoid receptor is released from this complex by the binding of steroids. The released receptor then migrates to the nucleus, where it binds to glucocorticoid response elements (GRE) in the regulatory regions of genes or modulates the binding of other transcription factors, such as AP-1, and the signal transducers and activators of transcription (STAT) factors. Also, the released glucocorticoid receptor inhibits other transcription factors, like nuclear factor

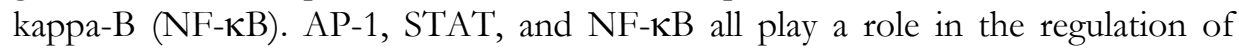
genes involved in the immune response. Binding of TAC to FKBP-52 may alter the affinity of the glucocorticoid receptor for steroids or may release the receptor from the complex and create a steroid mimetic effect. This may explain the steroid sparing effect of TAC compared to $\mathrm{CsA}^{81}$.

TAC also has an effect on cytokine formation induced by IL-2. It suppresses, independently of the calcineurin-NF-AT pathway, the production of IL-5 induced by IL-2 stimulation of the IL-2 receptor $^{82}$. In contrast to CsA, TAC was found to inhibit both native $T$-cells and primed T-cells in clinically relevant concentrations ${ }^{83}$. TAC was also able to enhance apoptosis (programmed cell death) in antigen-stimulated T-cells, whereas CsA had no effect. This effect is mediated by the downregulation of bcl- $\mathrm{x}_{\mathrm{L}}$, a member of the bcl-2 family ${ }^{84}$. Bcl-2 is an important regulator of apoptosis, preventing a cell from going into apoptosis. TAC potentiates T-cell apoptosis induced by steroids ${ }^{10}$. TAC also inhibits the migration of activated lymphocytes and the production of chemotactic factors by these cells ${ }^{85}$. In rats, TAC was able to reverse acute rejection, whereas CsA had no effect in equipotent dosages. In contrast to CsA, TAC appears to suppress intragraft IL-10 production and subsequent local infiltration of cytotoxic T-cells and natural killer cells by decreasing the release of such cytotoxic factors as 
granzyme B and perforin- ${ }^{86}$. The combination of the inhibition of primed T-cells, the induction of apoptosis, the inhibition of the migration of activated lymphocytes, and the suppression of IL-10 may explain the observation that TAC can reverse steroid-resistant rejections in patients receiving $\mathrm{CsA}^{87,88}$.

Another difference in efficacy may involve the effect on P-gp. Like CsA, TAC is eliminated from the cell by this protein ${ }^{50}$, but in contrast to CsA, TAC has not shown an inducible effect on P-gp in therapeutic concentrations ${ }^{49}$. In addition, the affinity of TAC for P-gp is less than that of CsA ${ }^{53}$. This may result in enhanced intracellular availability of TAC compared to CsA and, as a result, the efficacy may be increased. This effect may be a factor contributing to the reversal of acute rejections by $\mathrm{TAC}^{49}$. In supraphysiologic concentrations, however, TAC has also induced P-gp expression ${ }^{49}$.

In contrast to CsA, TAC inhibits inducible NO-synthase (NOS) gene expression during stimulation of the transcription regulator NF- $\mathrm{KB}$ in a calcineurinindependent way ${ }^{89}$, and TAC-treated patients have been shown to have lower levels of $\mathrm{NO}$ degradation products than CsA-treated patients ${ }^{13}$. However, in another cell line model, TAC appeared to have less of an effect on NO production than $\mathrm{CsA}^{47}$.

Like CsA and steroids, TAC is metabolized by the Cytochrome P-450 system, predominantly subtype $3 \mathrm{~A} 490,91$. Since both steroids and TAC are metabolized by CYP3A4, one cannot exclude possible interactions between the two drugs. Indeed, in dexamethasone-treated rats, the metabolism of TAC was found to be increased and CYP3A proteins were induced ${ }^{90,92}$. Also, the bioavailability of TAC shows a large intra- and inter-individual variability ${ }^{93}$; therefore, blood monitoring is needed to guide dosing between efficacy and toxicity ${ }^{94}$. Polymorphism of intestinal CYP3A proteins is a major factor contributing to the inter-individual variability ${ }^{95}$. In contrast to $\mathrm{CsA}$, which is highly bound to lipoproteins, TAC is predominantly bound to plasma proteins ${ }^{96}$.

Both CsA and TAC are nephrotoxic to the same extent histopathologically ${ }^{97}$. As mentioned before, FKBP-12 binds to subtype 1 of the TGF- $\beta$ receptor ${ }^{76,77}$. It acts as an anchor of another cytoplasmic protein to that receptor, probably calcineurin. The complex of FKBP-12 and calcineurin prevents the receptor from being activated $^{77}$. Macrolide derivatives of TAC, which can bind to FKBP-12 but have no effect on calcineurin, can release it from the TGF- $\beta$ type 1 receptor and enhance its activation ${ }^{77}$. However, TAC, which binds to FKBP-12 and inactivates calcineurin, has appeared to exhibit growth inhibition mediated by TGF- $\beta^{77}$. The downstream blockade of calcineurin and subsequent gene transcription is, therefore, essential for interruption of the effects of TGF- $\beta$. This may be beneficial with regard to the development of renal fibrosis. In contrast, CsA induces the expression of type 1 TGF- $\beta$ receptors ${ }^{65}$. In serum, TAC, like CsA, 
enhances the production of TGF- $\beta^{78}$. CsA and TAC may also have different effects on ET-1 secretion. Whereas CsA has been found to enhance the production, TAC appeared to have no effect on a cultured renal cell line ${ }^{98}$. However, another study did not find any difference in endothelin messenger RNA expression in renal biopsies ${ }^{99}$.

In contrast to $\mathrm{CsA}$, which induces hypertrichosis in a majority of patients, TAC is associated with hair loss in a minority of patients. It also does not cause gingival hyperplasia. Other side effects of TAC include gastro-intestinal disturbances, neurotoxicity, tremor, and pruritus ${ }^{100}$.

\section{Renal transplantation and cardiovascular disease}

Death with a functioning graft is still a major cause of graft loss after a successful renal transplantation. After the first year of transplantation, it is more common than graft rejection ${ }^{101}$. The main causes of mortality after renal transplantation are infections, malignancies, and cardiovascular disease, the latter being the most important ${ }^{101-103}$.

Nowadays, several independent risk factors for the development of cardiovascular disease have been established in the general population. Evaluation in this field started with the Framingham Heart Study ${ }^{104}$. To estimate the risk of cardiovascular disease with a combination of several risk factors, the Framingham risk score was developed. This score includes gender, age, smoking, diabetes mellitus, hypertension, and total and low-density lipoprotein cholesterol (LDL-c), and it correlates well with the incidence of cardiovascular disease and mortality ${ }^{105}$.

Thus far, prospective trials estimating the cardiovascular risk of the known population-based risk factors in the transplant population have not been performed; yet, it is unlikely that these risk factors have a more favourable impact after transplantation. On the contrary, the incidence of cardiovascular disease in the renal transplant population is more than four times greater than in the general population $^{106}$, and extrapolation of the risk of diabetes, smoking, and age established in the general population will underestimate the risk in the renal transplant population ${ }^{107}$. In addition, a definite risk factor for cardiovascular disease after transplantation is pre-existing disease ${ }^{106,108,109}$. Compared to the general population, patients with end-stage renal failure have a 10 to 20 times higher cardiovascular mortality ${ }^{110}$ and a very high cardiovascular morbidity. Renal transplant recipients have gone through this stage.

Moreover, several of the commonly known cardiovascular risk factors, such as hyperlipidemia, hypertension, diabetes mellitus, and renal function, are influenced by immunosuppressive agents. Prospective studies evaluating the effect of these 
drugs on firm cardiovascular endpoints, such as cardiovascular mortality, ischemic heart disease, and stroke, are, however, scarce. Hollander et al. ${ }^{111}$ reported an 8\% higher incidence of cardiovascular death eight years after transplantation in patients treated with CsA and steroids than in patients who were converted to AZA and steroids three months after transplantation. In the initial period after transplantation, TAC offered an increased risk of angina compared to CsA, but the overall incidence of cardiovascular disorders did not differ between the two drugs ${ }^{12,113}$. Since firm data are lacking, the effect of immunosuppressive drugs on population-based risk factors will be the target for optimization of immunosuppressive therapy in the renal transplant population.

\section{Post-transplant diabetes mellitus}

Traditionally, two main types of diabetes mellitus are distinguished: type 1 and, occurring approximately ten times more often, type 2 diabetes mellitus. Type 1 is mainly seen in young patients and is auto-immune-mediated, while type 2 is associated with obesity, increasing age, and several (partly known) genetic factors. Diabetes can also be related to the use of certain drugs, e.g., corticosteroidinduced diabetes mellitus, and may occur after organ transplantation (posttransplant diabetes mellitus, PTDM).

There is no single, clear definition of PTDM; consequently, many different ones have been used. Depending on the definition, incidences of approximately $5-40 \%$ have been reported in patients after renal transplantation ${ }^{114-116}$. Some investigators only include those patients using insulin treatment for a period of at least 30 days ${ }^{112}$, while others also include patients taking oral glucose-lowering drugs (need for drug treatment). Still others adhere to more stringent criteria, as proposed by the World Health Organization (WHO) or the American Diabetes Association $(\mathrm{ADA})^{117}$. In our clinical studies, we have defined PTDM as the need for treatment with either oral blood glucose-lowering drugs or insulin because this most closely reflects daily clinical practice.

A more subtle, subclinical, impairment of glucose metabolism can be detected with more sophisticated methods of investigation, such as the intravenous glucose tolerance test (iv-GTT) ${ }^{118,119}$ or euglycemic clamping. The latter technique is much more complicated and has higher costs. Therefore, the iv-GTT is the one we have used in our studies of glucose metabolism. It has enabled us to study impairment of insulin secretion and increases in insulin sensitivity, parameters of the development of diabetes mellitus. 
Several risk factors for the occurrence of PTDM have been described. These include genetic factors, race, family history of diabetes, older age, obesity, and the use of calcineurin inhibitors and steroids ${ }^{112-114,120-122}$.

Steroids disturb glucose tolerance by enhancing insulin resistance ${ }^{123}$. They play a significant role in the development of PTDM ${ }^{27,124}$. Withdrawal of steroids in CsAtreated patients has been shown to reduce the incidence of PTDM and to improve glycemic control ${ }^{125}$.

CsA also has an effect on glucose metabolism. Renal transplant patients using CsA and steroids were seen to have a higher incidence of PTDM than patients treated with azathioprine (AZA) and even higher dosages of steroids ${ }^{126}$. Reducing the dose of CsA reversed the diabetes in the majority of affected patients ${ }^{126}$. Glucose tolerance also improved after conversion from CsA to AZA ${ }^{127}$. The mechanism by which calcineurin inhibitors such as CsA and TAC may cause impaired glucose metabolism has not been completely elucidated. In vitro experiments have shown that CsA dose-dependently inhibited insulin secretion ${ }^{128,129}$.

In the initial report comparing CsA and TAC, it was mentioned that the incidence of PTDM was comparable ${ }^{130}$. The pivotal trials, however, showed significantly higher incidences with TAC ${ }^{112,113}$. The incidence of PTDM, as defined by the use of insulin for at least 30 days, was $20 \%$ with TAC versus $4 \%$ with CsA in the American trial ${ }^{112}$ and $11.6 \%$ versus $2.1 \%$, respectively, in the European trial ${ }^{113}$. The difference in diabetes incidence between the American and European studies may be explained by differences in the ethnic backgrounds of the groups studied. The higher risk of developing diabetes found in the American trial was due to the fact that the incidence in black and Hispanic patients is 3.3 times higher than that observed in Caucasian patients. In a majority of patients, PTDM was reversible after reducing the dose of TAC and withdrawing the steroids ${ }^{131}$. Especially those patients who already had sub-clinical impairment of glucose tolerance were at risk of developing PTDM with TAC ${ }^{132}$.

The mechanism responsible for the development of PTDM with TAC has been examined both in animals and in humans. An impaired insulin secretion during TAC administration was found in the former. Tamura et al. ${ }^{133}$ found a defect in insulin synthesis as a result of an mRNA transcriptional defect dependent on duration of exposure to TAC in rats. They hypothesized that the defect in mRNA transcription induced by TAC was mediated by binding to FKBP-12 and that the subsequent inhibition of calcineurin in $\beta$-cells caused the diminished insulin secretion. Others have described reduced insulin secretion in human pancreatic $\beta$-cells transplanted into animals 134,135 . Overexpression of P-gp induced by CsA compared to TAC, resulting in lower intracellular calcineurin inhibitor levels, could result in fewer abnormalities in glucose metabolism in CsA-treated patients ${ }^{136}$. Theoretically, from the binding of TAC to FKBP-52 in the glucocorticoid-receptor complex, an increased insulin resistance might be suspected due to liberation of glucocorticoid 
receptors, which could result in a steroid-mimetic effect ${ }^{81}$. In clinical studies, an increase in insulin resistance was suggested as an additional mechanism underlying the development of PTDM ${ }^{114}$. However, the results may be influenced by the concomitant use of steroids, which increase insulin resistance.

Of course, in addition to the effects of immunosuppressive agents, a decline in pancreatic function may also occur in transplant patients and contribute to the development of diabetes mellitus.

\section{Graft function}

The most important immunological reason for graft failure in the initial period after transplantation is acute rejection. Symptoms of acute rejection may be minimal, e.g., only an unexplained rise or insufficient decrease in serum creatinine, or may be accompanied by such symptoms as fever, tenderness of the graft, or hypertension. Decreased renal perfusion on a nuclear scan may reveal the acute rejection. Acute rejections are confirmed histologically by performing a renal biopsy. In the last decennium, consensus was reached to grade the severity of a rejection on histological grounds. The latest revision of these criteria was published in 1999, according to the 1997 consensus meeting in Banff, Canada ${ }^{137}$. In this latest version, a clear distinction was made between tubulo-interstitial rejections and vascular rejections (Table 1.1). The 1997 Banff classification correlates well with clinical outcome ${ }^{138-140}$. Tubulo-interstitial rejections have a better prognosis than vascular rejections ${ }^{138-141}$. Borderline rejections may improve spontaneously but progress, if untreated, in $28 \%$ to histological acute rejection. Progression of borderline rejection should especially be suspected if serum creatinine increases or is persistently elevated ${ }^{142}$.

The most important risk factors for acute rejections are mismatches in the human leukocyte antigen (HLA) system, especially mismatches in the HLA-DR locus ${ }^{143-145}$. Race, in this case being black, and age, in this case being young, are also associated with an increased risk of rejection ${ }^{144,146-150}$. Sensitized patients with antibodies to HLA antigens, as reflected in the percentage of panel-reactive antibodies (PRA), also have an enhanced risk of rejection ${ }^{14,151-153}$. Patients may become sensitized by former blood transfusions, pregnancies, or previous organ transplantations.

Acute rejections seldom occur after the initial six months post-transplantation, but when they do, these late acute rejections are associated with an impaired graft outcome ${ }^{154,155}$. Acute rejections that occur after the first two months posttransplantation are associated with an increased risk of chronic rejection and subsequent graft loss ${ }^{156}$. Disruption of immunosuppression may also provoke late acute rejections. 
Table 1.1 Distinction between tubulo-interstitial rejections and vascular rejections.

\begin{tabular}{|c|c|}
\hline Banff-criteria of acute rejection & Histopathological findings \\
\hline \multicolumn{2}{|l|}{ Borderline } \\
\hline \multirow[t]{3}{*}{ (suspicious of acute rejection) } & no intimal arteritis \\
\hline & $\begin{array}{l}\text { foci of mild tubulitis ( } 1-4 \text { mononuclear cells/tubular cross } \\
\text { section) }\end{array}$ \\
\hline & $10-25 \%$ of parenchyma inflamed \\
\hline \multicolumn{2}{|l|}{ Type I } \\
\hline \multirow[t]{8}{*}{ (tubulo-interstitial rejection) } & I A \\
\hline & $>25 \%$ of parenchyma inflamed \\
\hline & foci of moderate tubulitis ( $>4$ mononuclear cells/tubular cross \\
\hline & section or group of 10 tubular cells) \\
\hline & I B \\
\hline & $>25 \%$ of parenchyma inflamed \\
\hline & foci of severe tubulitis ( $>10$ mononuclear cells/tubular cross \\
\hline & section or group of 10 tubular cells) \\
\hline \multicolumn{2}{|l|}{ Type II } \\
\hline \multirow[t]{6}{*}{ (vascular rejection) } & II A \\
\hline & mild-to-moderate intimal arteritis in at least 1 arterial cross \\
\hline & section \\
\hline & II B \\
\hline & severe intimal arteritis with at least $25 \%$ luminal area loss in at \\
\hline & least 1 arterial cross section \\
\hline \multirow{5}{*}{$\begin{array}{l}\text { Type III } \\
\text { (severe transmural arterial changes) }\end{array}$} & \\
\hline & transmural arteritis \\
\hline & arterial fibrinoid change \\
\hline & necrosis of medial smooth muscle cells with lymphocytic \\
\hline & infiltrate in vessel \\
\hline
\end{tabular}

After the initial six months, a major cause of graft dysfunction and, ultimately, of graft loss, besides death with a functioning graft, is chronic transplant nephropathy, also called chronic allograft nephropathy (CAN). CAN is characterized by a gradual decrease in transplant function and the development of hypertension and proteinuria 157,158 . Histology shows vasculopathy: intimal thickening, smooth muscle cell proliferation, and multi-layering of peri-tubular capillary basement membranes, glomerulopathy, tubular atrophy, and interstitial fibrosis 159 . The cause of CAN cannot be determined by histology because histology is almost similar, irrespective of the predisposing factors. Renal function and proteinuria one year after transplantation are important surrogate markers for the development of CAN and graft survival160-164. Formerly, "chronic rejection" was the term most often used to denote this syndrome. Nowadays, this term is restricted to chronic graft dysfunction caused by immunological factors. Both 
cellular immunity and humoral immunity are involved in the development of chronic rejection ${ }^{165}$. Despite the involvement of immunological factors, enhancing immunosuppression does not resolve the syndrome.

In the last decennium, it became clear that several factors are involved in the development of $\mathrm{CAN}^{166}$. In Figure 1.1 the most important of these immunologic and non-immunologic factors are summarized. Acute rejections were already known to be a risk factor for $\mathrm{CAN}^{144,150,156,167}$. It has now become clear that especially recurrent acute rejections ${ }^{168}$ and vascular rejections are risk factors for $\mathrm{CAN}^{141}$ and that complete reversal of tubulo-interstitial rejection has little or no influence on graft survival ${ }^{169}$. Moreover, donor and transplant characteristics predispose to CAN. Graft survival is related to the number of nephrons transplanted ${ }^{170,171}$. Thus, kidneys derived from older, sub-optimal, or marginal donors perform less well than kidneys from optimal living donors ${ }^{172,173}$. Also, kidneys from male donors do better than those from female donors ${ }^{170}$. Ischemiareperfusion damage is also reflected in graft survival. The longer the period of ischemia, the more impaired graft survival will be ${ }^{172,174,175}$. Hyperfiltration, relative overflow in remaining nephrons, is an important factor in the development of $\mathrm{CAN}^{162}$. Cardiovascular risk factors such as hyper-tension ${ }^{176,177}$, hyperlipidemia ${ }^{178-}$ ${ }^{180}$, and diabetes mellitus ${ }^{172,181}$ all contribute to the development of CAN. It has been shown by Kooman et al that especially in patients with proteinuria (a hallmark of CAN), hypertension is often missed ${ }^{182}$. Nephrotoxicity by both calcineurin inhibitors, CsA and TAC, contribute to CAN as well.

\section{Chronic allograft nephropathy}

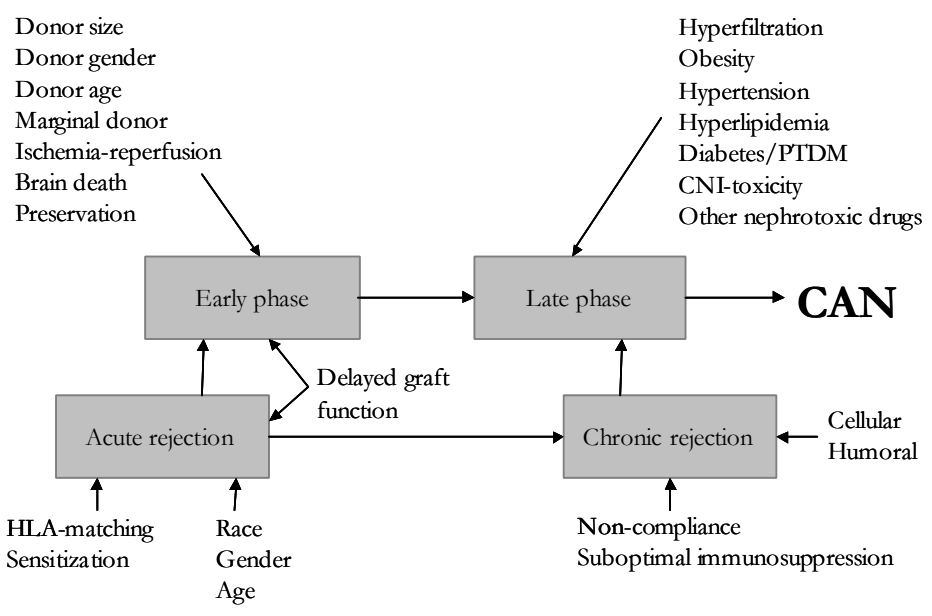

Figure 1.1 Schematic overview of factors involved in the development of Chronic Allograft Nephropathy (CAN). (Adapted from Pascual M et al. ${ }^{166}$ ) 
Since TAC has shown fewer acute rejections than CsA, it was suggested that graft survival would improve with TAC compared to CsA. However, this was not reflected in the comparable renal function one year post-transplantation in the main prospective trials ${ }^{112,113}$. Still, Gjertson et al. showed retrospectively in their analysis of the United States Organ Sharing (UNOS) database that graft survival might improve with TAC-based immunosuppression ${ }^{183}$. 


\section{Outline and aim of the thesis}

TAC had been proven to be a more potent immunosuppressive drug than CsASandimmune ${ }^{112,113}$. Certain cardiovascular risk factors have also tended to be less frequent in TAC-treated patients ${ }^{184}$. Yet, in 1995, a new micro-emulsion formulation of CsA was introduced into clinical practice (Neoral) ${ }^{33}$. This formulation resulted in a better and more consistent absorption of the drug; consequently, there was a lower incidence of acute rejections than with CsASandimmune ${ }^{185-187}$. The question then arose as to whether TAC would also be more potent than this micro-emulsion formulation.

At the start of this thesis, data comparing both drugs were almost absent. In an interim report, Morris-Stiff et al. ${ }^{188}$ showed that the results obtained with TAC and Neoral did not differ. However, their results may have been influenced by relatively low target levels of TAC. The efficacy of TAC is well correlated to its drug exposure, and low trough levels in the initial phase after transplantation are a risk factor for acute rejection ${ }^{189}$.

In the cyclosporin era, several attempts were made to withdraw steroids and to reduce the toxicity of these drugs. However, steroid withdrawal implied a risk of acute rejection and impaired graft survival ${ }^{125,190,191}$. Only Shapiro et al. ${ }^{131,192}$ attempted the withdrawal of steroids with TAC. They reported to have successfully achieved this in 50\% of their patients. Again, a 5\% risk of acute rejection was reported after withdrawal of the steroids, but these rejections responded to a re-institution of the steroids. Data about the effect of steroid withdrawal on graft survival with TAC have not been published.

The purpose of this thesis is to study the following:

TAC versus CsA-Neoral

1. Is there a difference in efficacy between TAC and CsA-Neoral in renal transplant recipients? (Chapter 2)

2. Is there a difference in transplant function and cardiovascular risk profile between TAC and CsA-Neoral in these patients? (Chapter 2)

Steroid withdrawal

1. If TAC appears to be more potent than CsA-Neoral, will it be possible to safely withdraw steroids? (Chapter 2)

2. Can steroids be safely withdrawn without increasing the risk of adrenal insufficiency in patients treated with TAC, mycophenolate mofetil, and steroids? (Chapter 3) 
3. Since both TAC and steroids are metabolized by the same cytochrome system in the liver, are TAC drug levels influenced by the concomitant use or withdrawal of steroids? (Chapter 4)

4. Is it feasible to withdraw steroids early after transplantation? (Chapter 5)

Cardiovascular risk factors

1. What is the contribution of both steroids and TAC to disturbed glucose metabolism after transplantation? (Chapter 6)

2. Is conversion from CsA to TAC safe in patients more than one year after transplantation? (Chapter 7)

3. Is it beneficial to CsA-treated patients to be converted to TAC more than one year after transplantation in terms of cardiovascular risk profile, renal function, and quality of life? (Chapters 7 and 8 )

4. What can we find in literature about the effects of the immunosuppressive agents currently used on cardiovascular risk factors? (Chapter 9) 


\section{References}

1. Wolfe RA, Ashby VB, Milford EL, Ojo AO, Ettenger RE, Agodoa LYC, Held PJ and Port FK. Comparison of mortality in all patients on dialysis, patients on dialysis awaiting transplantation, and recipients of a first cadaveric transplant. N Engl J Med 1999;341:1725-30.

2. Schnuelle P, Lorenz D, Trede M and Woude FJ van der. Impact of renal cadaveric transplantation on survival in end-stage renal failure: evidence for reduced mortality risk compared with hemodialysis during long-term follow-up. J Am Soc Nephrol 1998;9:2135-41.

3. Ojo AO, Hanson JA, Meier-Kriesche H-U, Okechukwu CN, Wolfe RA, Leichtman AB, Agodoa LY, Kaplan B and Port FK. Survival in recipients of marginal cadaveric donor kidneys compared with other recipients and wait-listed transplant candidates. J Am Soc Nephrol 2001; 12:589-97.

4. Waiser J, Budde K, Schreiber M, Peibst O, Koch U, Bohler T, Hoffken B, Hauser I and Neumayer HH. The quality of life in end stage renal disease care. Transpl Int 1998;11(S1): S42-5.

5. Karlberg I and Nyberg G. Cost-effectiveness studies of renal transplantation. Int J Technol Assess Health Care 1995;11:611-22.

6. Hench PS, Kendall EC, Slocumb CH and Polley HF. The effect of a hormone of the adrenal cortex (17-hydroxy-11-dehydrocortisone, compound 8E) and of pituitary adrenocorticotropic hormone on rheumatoid arthritis. Proc Staff Meet Mayo Clin 1949; 4:181-97.

7. Walsh JP and Dayan CM. Role of biochemical assessment in management of corticosteroid withdrawal. Ann Clin Biochem 2000;37:279-88.

8. Barnes PJ. Anti-inflammatory actions of glucocorticoids: molecular mechanisms. Clin Sci 1998; 94:557-72.

9. Bamberger CM, Bamberger A-M, Castro M de and Chrousos GP. Glucocorticoid receptor $B$, a potential endogenous inhibitor of glucocorticoid action in humans. J Clin Invest 1995;95: 2435-41.

10. Migita K, Eguchi K, Kawabe Y, Origuchi T, Tominaga M and Nagataki S. FK506 potentiates steroid-induced T-cell apoptosis. Transplantation 1997;64:1365-9.

11. Cronstein BN, Kimmel SC, Levin RI, Martiniuk F and Weissmann G. A machanism for the antiinflammatory effects of corticosteroids: The glucocorticoid receptor regulates leukocyte adhesion to endothelial cells and expression of endothelial-leukocyte adhesion molecule 1 and intercellular adhesion molecule 1. Proc Natl Acad Sci U S A 1992;89:9991-5.

12. Radomski MW, Palmer RMJ and Moncada S. Glucocorticoids inhibit the expression of an inducible, but not the constitutive, nitric oxide synthase in vascular endothelial cells. Proc Natl Acad Sci U S A 1990;87:10043-7.

13. Devlin J, Palmer RMJ, Gonde CE, O'Grady J, Heaton N, Tan KC, Martin JF, Moncada S and Williams R. Nitric oxide generation. A predicitve parameter of acute allograft rejection. Transplantation 1994;58:592-5.

14. AyanlarBatuman O, Ferrero AP, Diaz A and Jimenez SA. Regulation of transforming growth factor- $\$ 1$ gene expression by glucocorticoids in normal human $\mathrm{T}$ lymphocytes. J Clin Invest 1991;88:1574-80. 
15. Watkins PB, Murray SA, Winkelman LG, Heuman DM, Wrighton SA and Guzelian PS. Erythromycin breath test as an assay of glucocorticoid-inducible liver cytochromes P-450. Studies in rats and patients. J Clin Invest 1989;83:688-97.

16. Villikka K, Varis T, Backman JT, Neuvonen PJ and Kivistö KT. Effect of methylprednisolone on CYP3A4-mediated drug metabolism in vivo. Eur J Clin Pharmacol 2001;57:457-60.

17. McCune JS, Hawke RL, LeCluyse EL, Gillenwater HH, Hamilton G, Ritchie J and Lindley C. In vivo and in vitro induction of human cytochrome P4503A4 by dexamethasone. Clin Pharmacol Ther 2000;68:356-66.

18. Varis T, Backman JT, Kivistö KT and Neuvonen PJ. Diltiazem and mibefradil increase the plasma concentrations and greatly enhance the adrenal-suppressant effect of oral methylprednisolone. Clin Pharmacol Ther 2000;67:215-21.

19. Varis T, Kaukonen K-M, Kivistö KT and Neuvonen PJ. Plasma concentrations and effects of oral methylprednisolone are considerably increased by itraconazole. Clin Pharmacol Ther 1998; 64:363-8.

20. Spiegel RJ, Vigersky RA, Oliff AI, Echelberger CK, Bruton J and Poplack DG. Adrenal suppression after short-term corticosteroid therapy. Lancet 1979;1:630-3.

21. Wood JB, Frankland AW, James VHT and Landon J. A rapid test of adrenocortical function. Lancet 1965;1:243-5.

22. LaRochelle GE jr, LaRochelle AG, Ratner RE and Borenstein DG. Recovery of the hypothalamic-pituitary-adrenal (HPA) axis in patients with rheumatic diseases receiving lowdose prednisone. Am J Med 1993;95:258-64.

23. Kane KF, Emery P, Sheppard MC and Stewart PM. Assessing the hypothalamo-pituitaryadrenal axis in patients on long-term glucocorticoid therapy: the short synacthen versus the insulin tolerance test. Q J Med 1995;88:263-7.

24. Nichols T, Nugent CA and Tyler FH. Diurnal variation in suppression of adrenal function by glucocorticoids. J Clin Endocrinol Metab 1965;25:343-9.

25. Fraser CG, Preuss FS and Bigford WD. Adrenal atrophy and irreversible shock associated with cortisone therapy. J Am Med Assoc 1952;149:1542-3.

26. Lewis L, Robinson RF, Yee J, Hacker LA and Eisen G. Fatal adrenal cortical insufficiency precipitated by surgery during prolonged continuous cortisone infusion. Ann Intern Med 1953; 39:116-25.

27. Veenstra DL, Best JH, Hornberger J, Sullivan SD and Hricik DE. Incidence and long-term cost of steroid-related side effects after renal transplantation. Am J Kidney Dis 1999;33:829-39.

28. Bertoni E, Zanazzi M, Maria L di, Moscarelli L, Piperno R, Conti P, Dedola G, Bandini S, Tosi $\mathrm{P}$ and Salvadori M. Long-term steroid side effects in renal transplantation need a safe steroid withdrawal: a single-center experience. Transplant Proc 1998;30:1303-4.

29. Tarantino A, Montagnino G and Ponticelli C. Corticosteroids in kidney transplant recipients. Safety issues and timing of discontinuation. Drug Saf 1995;13:145-56.

30. Borel JF, Feurer C, Gubler HU and Stähelin H. Biological effects of cyclosporin A: a new antilymphocytic agent. Agents Actions 1976;6:468-75.

31. Sgoutas D, Macmahon W, Love A and Jerkunica I. Interaction of cyclosporin A with human lipoproteins. J Pharm Pharmacol 1986;38:583-8.

32. Groen PC de. Cyclosporine, low-density lipoprotein, and cholesterol. Mayo Clin Proc 1988;63: 1012-21.

33. Friman $\mathrm{S}$ and Bäckman L. A new microemulsion formulation of cyclosporin. Pharmacokinetic and clinical features. Clin Pharmacokinet 1996;30:81-93. 
34. Liu J, Farmer JD jr., Lane WS, Friedman J, Weissman I and Schreiber SL. Calcineurin is a common target of cyclophilin-cyclosporin A and FKBP-FK506 complexes. Cell 1991;66: $807-15$.

35. Halloran PF, Helms LMH, Kung L and Noujaim J. The temporal profile of calcineurin inhibition by cyclosporine in vivo. Transplantation 1999;68:1356-61.

36. O'Keefe SJ, Tamura J, Kincaid RL, Tocci MJ and O'Neill EA. FK-506- and CsA-sensitive activation of the interleukin-2 promotor by calcineurin. Nature 1992;357:692-4.

37. Clipstone NA and Crabtree GR. Identification of calcineurin as a key signalling enzyme in Tlymphocyte activation. Nature 1992;357:695-7.

38. Matsuda S and Koyasu S. Mechanisms of action of cyclosporine. Immunopharmacology 2000; 47:119-25.

39. Lang $\mathrm{Ph}$ and Baron C. Molecular mechanisms of immunosuppressive chemical agents recently introduced in clinical transplantation protocols. Nephrol Dial Transplant 1997;12:2050-4.

40. Schwaninger M, Blume R, Krüger M, Lux G, Oetjen E and Knepel W. Involvement of the $\mathrm{Ca} 2+$-dependent phosphatase calcineurin in gene transcription that is stimulated by cAMP through cAMP response elements. J Biol Chem 1995;270:8860-6.

41. Almawi WY and Melemedjian OK. Clinical and mechanistic differences between FK506 (tacrolimus) and cyclopsorin A. Nephrol Dial Transplant 2000;15:1916-8.

42. Matsuda S, Moriguchi T, Koyasu S and Nishida E. T lymphocyte activation signals for interleukin-2 production involve activation of MKK6-p38 and MKK7-SAPK/JNK signaling pathways sensitive to cyclosporin A. J Biol Chem 1998;273:12378-82.

43. Matsuda S, Shibasaki F, Takehana K, Mori H, Nishida E and Koyasu S. Two distinct action mechanisms of immunophilin-ligand complexes for the blockade of T-cell activation. Eur Mol Biol Org Reports 2000;1:428-34.

44. Li B, Sehajpal PK, Khanna A, Vlassara H, Cerami A, Stenzel KH and Suthanthiran M. Differential regulation of transforming growth factor $B$ and interleukin 2 genes in human $T$ cells: Demonstration by usage of novel competitor DNA constructs in the quantitative polymerase chain reaction. J Exp Med 1991;174:1259-62.

45. Khanna A, Li B, Stenzel KH and Suthanthiran M. Regulation of new DNA synthesis in mammalian cells by cyclosporine. Transplantation 1994;57:577-82.

46. Shin GT, Khanna A, Ding R, Sharma VK, Lagman M, Li B and Suthanthiran M. In vivo expression of transforming growth factor- $\beta 1$ in humans. Transplantation 1998;65:313-8.

47. Dusting GJ, Akita K, Hickey H, Smith M and Gurevich V. Cyclosporin A and tacrolimus (FK506) suppress expression of inducible nitric oxide synthase in vitro by different mechanisms. Br J Pharmacol 1999;128:337-44.

48. Jetté L, Beaulieu E, Leclerc J-M and Béliveau R. Cyclosporin A treatment induces overexpression of P-glycoprotein in the kidney and other tissues. Am J Physiol 1996;270: F756-65.

49. Hauser IA, Koziolek M, Hopfer U and Thévenod F. Therapeutic concentrations of cyclosporine A, but not FK506, increase P-glycoprotein expression in endothelial and renal tubule cells. Kidney Int 1998;54:1139-49.

50. Saeki T, Ueda K, Tanigawara Y, Hori R and Komano T. Human P-glycoprotein transports cyclosporin A and FK506. J Biol Chem 1993;268:6077-80.

51. Lo A and Burckart GJ. P-glycoprotein and drug therapy in organ transplantation. J Clin Pharmacol 1999;39:995-1005. 
52. Kemnitz J, Uysal A, Haverich A, Heublein B, Cohnert TR, Stangel W and Georgii A. Multidrug resistance in heart transplant patients: a preliminary communication on a possible mechanism of therapy-resistant rejection. J Heart Lung Transplant 1991;10:201-10.

53. Tanaka K, Hirai M, Tanigawara Y, Yasuhara M, Hori R, Ueda K and Inui K. Effect of cyclosporin analogues and FK506 on transcellular transport of daunorubicin and vinblastine via P-glycoprotein. Pharm Res 1996;13:1073-7.

54. Kahan BD, Welsh M, Schoenberg L, Rutzky LP, Katz SM, Urbauer DL and Buren CT van. Variable oral absorption of cyclosporine. A biopharmaceutical risk factor for chronic renal allograft rejection. Transplantation 1996;62:599-606.

55. Watkins PB. The role of cytochrome P-450 in cyclosporine metabolism. J Am Acad Dermatol 1990;23:1301-11.

56. Watkins PB, Hamilton TA, Annesley TM, Ellis CN, Kolars JC and Voorhees JJ. The erythromycin breath test as a predictor of cyclosporine blood levels. Clin Pharmacol Ther 1990;48:120-9.

57. Jeng S, Chanchairujira T, Jusko W and Steiner R. Prednisone metabolism in recipients of kidney or liver transplants and in lung recipients receiving ketoconazol. Transplantation 2003; 75:792-5.

58. Curtis JJ, Luke RG, Dubovsky E, Diethelm AG, Whelchel JD and Jones P. Cyclosporin in therapeutic doses increases renal allograft vascular resistance. Lancet 1986;2:477-9.

59. Ishikawa A, Suzuki K and Fujita K. Mechanisms of Cyclosporine-induced nephrotoxicity. Transplant Proc 1999;31:1127-8.

60. Lanese DM and Conger JD. Effects of endothelin receptor antagonist on cyclosporine-induced vasoconstriction in isolated rat renal arterioles. J Clin Invest 1993;91:2144-9.

61. Kurihara H, Yoshizumi M, Sugiyama T, Takaku F, Yanagisawa M, Masaki T, Hamaoki M, Kato $\mathrm{H}$ and Yazaki Y. Transforming growth factor- $\beta$ stimulates the expression of endothelin mRNA by vascular endothelial cells. Biochem Biophys Res Com 1989;159:1435-40.

62. Chareandee C, Herman WH, Hricik DE and Simonson MS. Elevated endothelin-1 in tubular epithelium is associated with renal allograft rejection. Am J Kidney Dis 2000;36:541-9.

63. Simonson MS, Emancipator SN, Knauss T and Hricik DE. Elevated neointimal endothelin-1 in transplantation-associated arteriosclerosis of renal allograft recipients. Kidney Int 1998;54: 960-71.

64. Simonson MS, Herman WH, Robinson A, Schulak J and Hricik DE. Inhibition of endothelinconverting enzyme attenuates transplant vasculopathy and rejection in rat cardiac allografts. Transplantation 1999;67:1542-7.

65. Waiser J, Dell K, Böhler T, Dogu E, Gaedeke J, Budde K and Neumayer H-H. Cyclosporine A up-regulates the expression of TGF- $\beta 1$ and its receptors type I and type II in rat mesangial cells. Nephrol Dial Transplant 2002;17:1568-77.

66. Yamamoto T, Noble NA, Miller DE and Border WA. Sustained expression of TGF-B1 underlies development of progressive kidney fibrosis. Kidney Int 1994;45:916-27.

67. Sharma VK, Bologa RM, Xu G-P, Li B, Mouradian J, Wang J, Serur D, Rao V and Suthanthiran M. Intragraft TGF-B1 mRNA: A correlate of interstitial fibrosis and chronic allograft nephropathy. Kidney Int 1996;49:1297-303.

68. Thomas SE, Andoh TF, Pichler RH, Shankland SJ, Couser WG, Bennett WM and Johnson RJ. Accelerated apoptosis characterizes cyclosporine-associated interstitial fibrosis. Kidney Int 1998;53:897-908. 
69. Bakker RC, Kooten C van, Lagemaat-Paape ME van de, Daha MR and Paul LC. Renal tubular epithelial cell death and cyclosporin A. Nephrol Dial Transplant 2002;17:1181-8.

70. Paus R, Foitzik K, Welker P, Bulfone-Paus S and Eichmüller S. Transforming growth factor- $\beta$ receptor type I and type II expression during murine hair follicle development and cycling. J Invest Dermatol 1997;109:518-26.

71. Stabellini G, Carinci F, Bedani PL, Calastrini C, Mattei M de, Scapoli L, Caruso A, Calvitti M and Locci P. Cyclosporin A and transforming growth factor $B$ modify the pattern of extracellular glycosaminoglycans without causing cytoskeletal changes in human gingival fibroblasts. Transplantation 2002;73:1676-9.

72. Hojo M, Morimoto T, Maluccio M, Asano T, Morimoto K, Lagman M, Shimbo T and Suthanthiran M. Cyclosporine induces cancer progression by a cell-autonomous mechanism. Nature 1999;397:530-4.

73. Goto T, Kino T, Hatanaka H, Nishiyama M, Okuhara M, Kohsaka M, Aoki H and Imanaka H. Discovery of FK-506, a novel immunosuppressant isolated from Streptomyces tsukubaensis. Transplant Proc 1987;19:4-8.

74. Xu X, Su B, Barndt RJ, Chen H, Xin H, Yan G, Chen L, Cheng D, Heitman J, Zhuang Y, Fleischer S and Shou W. FKBP12 is the only FK506 binding protein mediating T-cell inhibition by the immunosuppressant FK506. Transplantation 2002;73:1835-8.

75. Cameron AM, Steiner JP, Roskams AJ, Ali SM, Ronnett GV and Snyder SH. Calcineurin associated with the inositol 1,4,5-triphosphate receptor-FKBP12 complex modulates Ca2+ flux. Cell 1995;83:463-72.

76. Wang T, Donahoe PK and Zervos AS. Specific interaction of type I receptors of the TGF- $B$ family with the immunophilin FKBP-12. Science 1994;265:674-6.

77. Wang T, Li B-Y, Danielson PD, Shah PC, Rockwell S, Lechleider RJ, Martin J, Manganaro T and Donahoe PK. The immunophilin FKBP12 functions as a common inhibitor of the TGF- $\beta$ family type 1 receptors. Cell 1996;86:435-44.

78. Khanna A, Cairns V and Hosenpud JD. Tacrolimus induces increased expression of transforming growth factor- $\beta 1$ in mammalian lymphoid as well as nonlymphoid cells. Transplantation 1999;67:614-9.

79. Suzuki N, Sakane T and Tsunematsu T. Effects of a novel immunosuppressive agent, FK506, on human B cell activation. Clin Exp Immunol 1990;79:240-5.

80. Jurcevic S, Dunn MJ, Crisp S, Busing K, Rinaldi M, Pellegrini C, Yacoub MH, Vigano M, Banner NL and Rose ML. A new enzyme-linked immunosorbent assay to measure antiendothelial antibodies after cardiac transplantation demonstrates greater inhibition of antibody formation by tacrolimus compared with cyclosporine. Transplantation 1998;65:1197-202.

81. Hutchinson IV, Bagnall W, Bryce P, Pufong B, Geraghty P and Brogan I. Differences in the mode of action of cyclosporine and FK 506. Transplant Proc 1998;30:959-60.

82. Mori A, Suko M, Kaminuma O, Inoue S, Ohmura T, Hoshino A, Asakura Y, Terada E, Miyazawa K, Nosaka C, Okumura Y, Ito K and Okudaira H. IL-2-induced IL-5 synthesis, but not proliferation, of human CD4+ T cells is suppressed by FK506. J Immunol 1997;158: 3659-65.

83. Roelen DL, Bree FPMJ van, Schanz U, Rood JJ van and Claas FHJ. Differential inhibition of primed alloreactive CTLs in vitro by clinically used concentrations of cyclosporine and FK506. Transplantation 1993;56:190-5. 
84. Migita K, Origuchi T, Kawabe Y, Tominaga M, Ida H, Kawakami A and Eguchi K. FK506 markedly enhances apoptosis of antigen-stimulated peripheral T-cells by down-regulation of Bcl-xL. Transplantation 1999;68:1018-23.

85. Adams DH and Liu Q. FK506 inhibits human lymphocyte migration and the production of lymphocyte chemotactic factors in liver allograft recipients. Hepatology 1996;23:1476-83.

86. Jiang H, Wynn C, Pan F, Ebbs A, Erickson LM and Kobayashi M. Tacrolimus and cyclosporine differ in their capacity to overcome ongoing allograft rejection as a result of their differential abilities to inhibit interleukin-10 production. Transplantation 2002;73:1808-17.

87. Woodle ES, Thistlethwaite JR, Gordon JH, Laskow D, Deierhoi MH, Burdick J, Pirsch JD, Sollinger H, Vincenti F, Burrows L, Schwartz B, Danovitch GM, Wilkinson AH, Shaffer D, Simpson MA, Freeman RB, Rohrer RJ, Mendez R, Aswad S, Munn SR, Wiesner RH, Delmonico FL, Neylan J and Whelchel J. A multicenter trial of FK506 (tacrolimus) therapy in refractory acute renal allograft rejection. A report of the Tacrolimus Kidney Transplantation Rescue Study Group. Transplantation 1996;62:594-9.

88. Jordan ML, Naraghi R, Shapiro R, Smith D, Vivas CA, Scantlebury VP, Gritsch HA, McCauley J, Randhawa P, Demetris AJ, McMichael J, Fung JJ and Starzl TE. Tacrolimus rescue therapy for renal allograft rejection - five-year experience. Transplantation 1997;63:223-8.

89. Kaibori M, Sakitani K, Oda M, Kamiyama Y, Masu Y, Nishizawa M, Ito S and Okumura T. Immunosuppressant FK506 inhibits inducible nitric oxide synthase gene expression at a step of NK-kB activation in rat hepatocytes. J Hepatol 1999;30:1138-45.

90. Sattler M, Guengerich FP, Yun CH, Christians U and Sewing KF. Cytochrome P-450 3A enzymes are responsible for biotransformation of FK506 and rapamycin in man and rat. Drug Metab Dispos 1992;20:753-61.

91. Shiraga T, Matsuda H, Nagase K, Iwasaki K, Noda K, Yamazaki H, Shimada T and Funae Y. Metabolism of FK506, a potent immunosuppressive agent, by cytochrome P450 3A enzymes in rat, dog and human liver microsomes. Biochem Pharmacol 1994;47:727-35.

92. Shimada T, Terada A, Yokogawa K, Kaneko H, Nomura M, Kaji K, Kaneko S, Kobayashi K and Miyamoto K. Lowered blood concentration of tacrolimus and its recovery with changes in expression of CYP3A and P-glycoprotein after high-dose steroid therapy. Transplantation 2002;74:1419-24.

93. Venkataramanan R, Jain A, Warty VS, Abu-Elmagd K, Alessiani M, Lever J, Krajak A, Flowers J, Mehta S, Zuckerman S, Fung J, Todo S and Starzl TE. Pharmacokinetics of FK 506 in transplant patients. Transplant Proc 1991;23:2736-40.

94. Undre NA, Stevenson P and Schäfer A. Pharmacokinetics of tacrolimus: Clinically relevant aspects. Transplant Proc 1999;31(S7A): 1S-4.

95. Macphee IA, Fredericks S, Tai T, Syrris P, Carter ND, Johnston A, Goldberg L and Holt DW. Tacrolimus pharmacogenetics: polymorphisms associated with expression of cytochrome p4503A5 and P-glycoprotein correlate with dose requirment. Transplantation 2002;74:1486-9.

96. Warty V, Venkataramanan R, Zendehrouh P, McKaveney T, Chao J, Todo S and Starzl T. Distribution of FK 506 in plasma lipoproteins in transplant patients. Transplant Proc 1991;23: 954-5.

97. Mihatsch MJ, Kyo M, Morozumi K, Yamaguchi Y, Nickeleit V and Ryffel B. The side effects of ciclosporin-A and tacrolimus. Clin Nephrol 1998;49:356-63.

98. Nakahama H, Fukunaga M, Kakihara M, Horio M, Fujiwara Y, Fukuhara Y, Ueda N, Orita Y and Kamada T. Comparative effect of cyclosporine A and FK-506 on endothelin secretion by a cultured renal cell line, LLC-PK1. J Cardiovasc Pharmacol 1991;17(S7):S172-3. 
99. Kirk AD, Jacobson LM, Heisey DM, Fass NA, Sollinger HW and Pirsch JD. Posttransplant diastolic hypertension. Associations with intragraft transforming growth factor- $\beta$, endothelin and renin transcription. Transplantation 1997;64:1716-20.

100. Plosker GL and Foster RH. Tacrolimus. A further update of its pharmacology and therapeutic use in the management of organ transplantation. Drugs 2000;59:323-89.

101. Lindholm A, Albrechtsen D, Frödin L, Tufveson G, Persson NH and Lundgren G. Ischemic heart disease - Major cause of death and graft loss after renal transplantation in Scandinavia. Transplantation 1995;60:451-7.

102. Howard RJ, Patton PR, Reed AI, Hemming AW, Werf WJ van der, Pfaff WW, Srinivas TR and Scornik JC. The changing causes of graft loss and death after kidney transplantation. Transplantation 2002;73:1923-8.

103. Ojo AO, Hanson JA, Wolfe RA, Leichtman AB, Agodoa LY and Port FK. Long-term survival in renal transplant recipients with graft function. Kidney Int 2000;57:307-13.

104. Dawber TR and Kannel WB. The Framingham study. An epidemiological approach to coronary heart disease. Circulation 1966;34:553-5.

105. Wilson PWF, D'Agostino RB, Levy D, Belanger AM, Silbershatz H and Kannel WB. Prediction of coronary heart disease using risk factor categories. Circulation 1998;97:1837-47.

106. Kasiske BL. Risk factors for accelerated atherosclerosis in renal transplant recipients. Am J Med 1988;84:985-92.

107. Kasiske BL, Chakkera HA and Roel J. Explained and unexplained ischemic heart disease risk after renal transplantation. J Am Soc Nephrol 2000;11:1735-43.

108. Manske CL, Wang Y, Rector T, Wilson RF and White CW. Coronary revascularisation in insulin-dependent diabetic patients with chronic renal failure. Lancet 1992;340:998-1002.

109. Kasiske BL, Guijarro C and Massy ZA. Cardiovascular disease after renal transplantation. J Am Soc Nephrol 1996;7:158-65.

110. Foley RN, Parfrey PS and Sarnak MJ. Clinical epidemiology of cardiovascular disease in chronic renal disease. Am J Kidney Dis 1998;32(5S3):S112-9.

111. Hollander AA, Saase JL van, Kootte AM, Dorp WT van, Bockel HJ van, Es LA van and Woude FJ van der. Beneficial effects of conversion from cyclosporin to azathioprine after kidney transplantation. Lancet 1995;345:610-4.

112. Pirsch JD, Miller J, Deierhoi MH, Vincenti F and Filo RS for the FK506 Kidney Transplant Study Group. A comparison of tacrolimus (FK506) and cyclosporine for immunosuppression after cadaveric renal transplantation. Transplantation 1997;63:977-83.

113. Mayer AD, Dmitrewski J, Squifflet J-P, Besse T, Grabensee B, Klein B, Eigler FW, Heemann U, Pichlmayr R, Behrend M, Vanrenterghem Y, Donck J, Hooff J van, Christiaans M, Morales JM, Andres A, Johnson RWG, Short C, Buchholz B, Rehmert N, Land W, Schleibner S, Forsythe JLR, Talbot D, Neumayer H-H, Hauser I, Ericzon B-G, Brattström C, Claesson K, Mühlbacher F and Pohanka E. Multicenter randomized trial comparing tacrolimus (FK 506) and cyclosporin in the prevention of renal allograft rejection. A report of the European Tacrolimus Multicenter Renal Study Group. Transplantation 1997;64:436-43.

114. Weir MR and Fink JC. Risk for posttransplant diabetes mellitus with current immunosuppressive medications. Am J Kidney Dis 1999;34:1-13.

115. Krentz AJ, Dmitrewski J, Mayer D and Natrass M. Effects of immunosuppressive agents on glucose metabolism. Clin Immunother 1995;4:103-23.

116. Jindal RM, Sidner RA and Milgrom ML. Post-transplant diabetes mellitus. The role of immunosuppression. Drug Saf 1997;16:242-57. 
117. Expert Committee on the Diagnosis and Classification of Diabetes Mellitus. American Diabetes Association: clinical practice recommendations 2002. Diabetes Care 2002;25(S1):S1-147.

118. Bergman RN, Finegood DT and Ader M. Assessment of insulin sensitivity in vivo. Endocrine Rev 1985;6:45-86.

119. Elmer DS, Hathaway DK and Gaber AO. Monitoring of pancreas allograft function with glucose disappearance rate $(\mathrm{KG})$ : calculation and interpretation. J Transplant Coordination 1994;4:7-11.

120. Sumrani NB, Delaney V, Ding ZK, Davis R, Daskalakis P, Friedman EA, Butt KM and Hong JH. Diabetes mellitus after renal transplantation in the cyclosporine era - an analysis of risk factors. Transplantation 1991;51:343-7.

121. Hjelmesaeth J, Hartman A, Kofstad J, Stenstrom J, Leivestad T, Egeland T and Fauchald P. Glucose intolerance after renal transplantation depends upon prednisolone dose and recipient age. Transplantation 1997;64:979-83.

122. Cosio FG, Pesavento TE, Osei K, Henry ML and Ferguson RM. Post-transplant diabetes mellitus. Increasing incidence in renal allograft recipients transplanted in recent years. Kidney Int 2001;59:732-7.

123. Pagano G, Bruno A, Cavallo-Perin P, Cesco L and Imbimbo B. Glucose intolerance after short-term administration of corticosteroids in healthy subjects. Prednisone, deflazacort, and betamethasone. Arch Intern Med 1989;149:1098-101.

124. Hricik DE, Bartucci MR, Moir EJ, Mayes JT and Schulak JA. Effects of steroid withdrawal on posttransplant diabetes mellitus in cyclosporine-treated renal transplant recipients. Transplantation 1991;51:374-7.

125. Hollander AAMJ, Hené RJ, Hermans J, Es LA van and Woude FJ van der. Late prednisone withdrawal in cyclosporine-treated kidney transplant patients: a randomized study. J Am Soc Nephrol 1997;8:294-301.

126. Yamamoto H, Akazawa S, Yamaguchi Y, Yokota A, Yamasaki H, Nakanishi T, Tahara D, Matsuya F, Saito Y and Nagataki S. Effects of cyclosporin A and low dosages of steroid on posttransplantation diabetes in kidney transplant recipients. Diabetes Care 1991;14:867-70.

127. Harris KP, Russell GI, Parvin SD, Veitch PS and Walls J. Alterations in lipid and carbohydrate metabolism attributable to cyclosporin A in renal transplant recipients. Br Med J 1986;292: 16-9.

128. Robertson RP. Cyclosporin-induced inhibition of insulin secretion in isolated rat islets and HIT cells. Diabetes 1986;35:1016-9.

129. Gillison SL, Bartlett ST and Curry DL. Inhibition by cyclosporine of insulin secretion--a beta cell-specific alteration of islet tissue function. Transplantation 1991;52:890-5.

130. Shapiro R, Jordan M, Scantlebury V, Fung J, Jensen C, Tzakis A, McCauley J, Carroll P, Ricordi C, Demetris AJ, Mitchell S, Jain A, Iwaki Y, Kobayashi M, Reyes J, Todo S, Hakala TR, Simmons RL and Starzl TE. FK 506 in clinical kidney transplantation. Transplant Proc 1991;23:3065-7.

131. Shapiro R, Jordan ML, Scantlebury VP, Vivas C, Fung JJ, McCauley J, Randhawa P, Demetris AJ, Irish W, Mitchell S, Hakala TR, Simmons RL and Starzl TE. A prospective randomized trial of FK506-based immunosuppression after renal transplantation. Transplantation 1995;59: 485-90.

132. Duijnhoven EM van, Boots JMM, Christiaans MHL, Wolffenbuttel BHR and Hooff JP van. Influence of tacrolimus on glucose metabolism before and after renal transplantation: a prospective study. J Am Soc Nephrol 2001;12:583-8. 
133. Tamura K, Fujimura T, Tsutsumi T, Nakamura K, Ogawa T, Atumaru C, Hirano Y, Ohara K, Ohtsuka K and Shimomura K. Transcriptional inhibition of insulin by FK506 and positive involvement of FK506 binding protein-12 in pancreatic beta-cell. Transplantation 1995;59: 1606-13.

134. Ricordi C, Zeng Y, Alejandro R, Tzakis A, Venkataramanan R, Fung J, Bereiter D, Mintz D and Starzl T. In vivo effect of FK506 on human pancreatic islets. Transplantation 1991;52: 519-22.

135. Rilo H, Zeng Y, Alejandro R, Carroll P, Bereiter D, Venkataramanan R, Tzakis A, Starzl T and Ricordi C. Effect of FK506 on function of human islets of Langerhans. Transplant Proc 1991; 23:3164-5.

136. Zanker B, Barth C, Stachowski J, Baldamus CA and Land W. Multidrug resistance gene MDR1 expression: a gene transfection in vitro model and clinical analysis in cyclosporine-treated patients rejecting their renal grafts. Transplant Proc 1997;29:1507-8.

137. Racusen LC, Solez K, Colvin RB, Bonsib SM, Castro MC, Cavallo T, Croker BP, Demetris AJ, Drachenberg CB, Fogo AB, Furness P, Gaber LW, Gibson IW, Glotz D, Goldberg JC, Grande JG, Halloran PF, Hansen HE, Hartley B, Hayry PJ, Hill CM, Hoffman EO, Hunsicker LG, Lindblad AS, Marcussen N, Mihatsch MJ, Nadasdy T, Nickerson P, Olsen TS, Papadimitriou JC, Randhawa PS, Rayner DC, Roberts I, Rose S, Rush D, Salinas-Madrigal L, Salomon DR, Sund S, Taskinen E, Trpkov K and Yamaguchi Y. The Banff 97 working classification of renal allograft pathology. Kidney Int 1999;55:713-23.

138. Macdonald FI, Ashraf S, Picton M, Dyer PA, Parrott NR, Short CD and Roberts ISD. Banff criteria as predictors of outcome following acute renal allograft rejection. Nephrol Dial Transplant 1999;14:1692-7.

139. Bates WD, Davies DR, Welsh K, GRay DWR, Fuggle SV and Morris PJ. An evaluation of the Banff classification of early renal allograft biopsies and correlation with outcome. Nephrol Dial Transplant 1999;14:2364-9.

140. Mueller A, Schnuelle P, Waldherr R and Woude FJ van der. Impact of the Banff ' 97 classification for histological diagnosis of rejection on clinical outcome and renal function parameters after kidney transplantation. Transplantation 2000;69:1123-7.

141. Saase JLCM, Woude FJ van der, Thorogood J, Hollander AAMJ, Es LA van, Weening JJ, Bockel JH van and Bruijn JA. The relation between acute vascular and interstitial renal allograft rejection and subsequent chronic rejection. Transplantation 1995;59:1280-5.

142. Meehan SM, Siegel CT, Aronson AJ, Bartosh SM, Thistlethwaite JR, Woodle ES and Haas M. The relationship of untreated borderline infiltrates by the Banff criteria to acute rejection in renal allograft biopsies. J Am Soc Nephrol 1999;10:1806-14.

143. Pirsch JD, Ploeg RJ, Gange S, D'Alessandro AM, Knechtle SJ, Sollinger HW, Kalayoglu M and Belzer FO. Determinants of graft survival after renal transplantation. Transplantation 1996;61: 1581-6.

144. Lindholm A, Ohlman S, Albrechtsen D, Tufveson G, Persson H and Persson NH. The impact of acute rejection episodes on long-term graft function and outcome in 1347 primary renal transplants treated by 3 cyclosporine regimens. Transplantation 1993;56:307-15.

145. Richards E, Schleibner S, Talbot D and the European Tacrolimus Multicentre Renal Study Group. An exploratory analysis of prognostic factors for patient outcome during the first year following renal transplantation. Transplant Proc 1998;30:1386-8. 
146. Ishikawa A, Flechner SM, Goldfarb DA, Myles JL, Modlin CS, Boparai N, Papajcik D, Mastroianni B and Novick AC. Quantitative assessment of the first acute rejection as a predictor of renal transplant outcome. Transplantation 1999;68:1318-24.

147. Isaacs RB, Nock SL, Spencer CE, Connors AF jr, Wang X-Q, Sawyer R and Lobo PI. Racial disparities in renal transplant outcomes. Am J Kidney Dis 1999;34:706-12.

148. Neylan JF and for the US Renal Transplant Mycophenolate Mofetil Study Group. Immunosuppressive therapy in high-risk transplant patients. Dose-dependent efficacy of mycophenolate mofetil in African-American renal allograft recipients. Transplantation 1997; 64:1277-82.

149. Roodnat JI, Zietse R, Mulder PGH, Rischen-Vos J, Gelder T van, IJzermans JNM and Weimar W. The vanishing importance of age in renal transplantation. Transplantation 1999;67:576-80.

150. Humar A, Hassoun A, Kandaswamy R, Payne WD, Sutherland DER and Matas AJ. Immunologic factors: the major risk for decreased long-term renal allograft survival. Transplantation 1999;68:1842-6.

151. Chapman JR, Taylor CJ, Ting A and Morris PJ. Immunoglobulin class and specificity of antibodies causing positive $\mathrm{T}$ cell crossmatches. Relationship to renal transplant outcome. Transplantation 1986;42:608-13.

152. Taylor CJ, Chapman JR, Ting A and Morris PJ. Characterization of lymphocytotoxic antibodies causing a positive crossmatch in renal transplantation. Transplantation 1989;48: 953-8.

153. Sanfilippo F, Vaughn WK, LeFor WM and Spees EK. Multivariate analysis of risk factors in cadaver donor kidney transplantation. Transplantation 1986;42:28-34.

154. Humar A, Kerr S, Gillingham KJ and Matas AJ. Features of acute rejection that increase risk for chronic rejection. Transplantation 1999;68:1200-3.

155. Sijpkens Y, Doxiadis I, Mallat M, Fijter H de, Bruijn JA, Claas F and Paul L. Early versus late acute rejection episodes in renal transplantation. Transplantation 2003;75:204-8.

156. Basadonna GP, Matas AJ, Gillingham KJ, Payne WD, Dunn DL, Sutherland DER, Gores PF, Gruessner RWG and Najarian JS. Early versus late acute renal allograft rejection: impact on chronic rejection. Transplantation 1993;55:993-5.

157. Halloran PF, Melk A and Barth C. Rethinking chronic allograft nephropathy: the concept of accelerated senescence. J Am Soc Nephrol 1999;10:167-81.

158. Monaco AP, Burke JF jr, Ferguson RM, Halloran PF, Kahan BD, Light JA, Matas AJ and Solez K. Current thinking on chronic renal allograft rejection: issues, concerns, and recommendations from a 1997 roundtable discussion. Am J Kidney Dis 1999;33:150-60.

159. Kasiske BL, Kalil RS, Lee HS and Rao KV. Histopathologic findings associated with a chronic, progressive decline in renal allograft function. Kidney Int 1991;40:514-24.

160. Hariharan S, McBride MA, Cherikh WS, Tolleris CB, Bresnahan BA and Johnson CP. Posttransplant renal function in the first year predicts long-term kidney transplant survival. Kidney Int 2002;62:311-8.

161. Sund S, Reisæter AV, Fauchald P, Bentdal $\varnothing$, Sundby Hall K and Hovig T. Living donor kidney transplants: a biopsy study 1 year after transplantation, compared with baseline changes and correlation to kidney function at 1 and 3 years. Nephrol Dial Transplant 1999;14:2445-54.

162. Terasaki PI, Koyama H, Cecka JM and Gjertson DW. The hyperfiltration hypothesis in human renal transplantation. Transplantation 1994;57:1450-4. 
163. Alcalde G, Escallada R, Cotorruelo JG, Zubimendi JA, Francisco ALM de and Arias M. Disproportion between kidney graft and recipient size is the main predictor of long-term proteinuria. Transplant Proc 1997;29:127-8.

164. Nankivell BJ, Fenton-Lee CA, Kuypers DRJ, Cheung E, Allen RDM, O'Connell PJ and Chapman JR. Effect of histological damage on long-term kidney transplant outcome. Transplantation 2001;71:515-23.

165. Joosten SA, Kooten C van and Paul LC. Pathogenesis of chronic allograft rejection. Transpl Int 2003;16:137-45.

166. Pascual M, Theruvath T, Kawai T, Tolkoff-Rubin N and Cosimi AB. Strategies to improve long-term outcomes after renal transplantation. N Engl J Med 2002;346:580-90.

167. Matas A. Chronic rejection in renal transplant recipients - risk factors and correlates. Clin Transplant 1994;8:332-5.

168. Humar A, Payne WD, Sutherland DER and Matas AJ. Clinical determinants of multiple acute rejection episodes in kidney transplant recipients. Transplantation 2000;69:2357-60.

169. Madden RL, Mulhern JG, Benedetto BJ, O'Shea MH, Germain MJ, Braden GL, O'Shaughnessy J and Lipkowitz GS. Completely reversed acute rejection is not a significant risk factor for the development of chronic rejection in renal allograft recipients. Transpl Int 2000;13:344-50.

170. Brenner BM, Cohen RA and Milford EL. In renal transplantation, one size may not fit all. J Am Soc Nephrol 1992;3:162-9.

171. Kasiske BL, Snyder JJ and Gilbertson D. Inadequate donor size in cadaver kidney transplantation. J Am Soc Nephrol 2002;13:2152-9.

172. Morris PJ, Johnson RJ, Fuggle SV, Belger MA and Briggs JD on behalf of the HLA Task Force of the Kidney Advisory Group of the United Kingdom Transplant Support Service Authority (UKTSSA). Analysis of factors that affect outcome of primary cadaveric renal transplantation in the UK. Lancet 1999;354:1147-52.

173. Nickerson P, Jeffery J, Gough J, McKenna R, Grimm P, Cheang M and Rush D. Identification of clinical and histopathologic risk factors for diminished renal function 2 years posttransplant. J Am Soc Nephrol 1998;9:482-7.

174. Mange KC, Cherikh WS, Maghirang J and Bloom RD. A comparison of the survival of shipped and locally transplanted cadaveric renal allografts. N Engl J Med 2001;345:1237-42.

175. Asderakis A, Dyer P, Augustine T, Worthington J, Campbell B and Johnson RWG. Effect of cold ischemia time and HLA matching in kidneys coming from "young" and "old" donors. Do not leave for tomorrow what you can do tonight. Transplantation 2001;72:674-8.

176. Opelz G, Wujciak T, Ritz E and for the Collaborative Transplant Study. Association of chronic kidney graft failure with recipient blood pressure. Kidney Int 1998;53:217-22.

177. Mange KC, Cizman B, Joffe M and Feldman HI. Arterial hypertension and renal allograft survival. J Am Med Assoc 2000;283:633-8.

178. Peschke B, Scheuermann, Geiger H, Bölscher S, Kachel H-G and Lenz T. Hypertension is associated with hyperlipidemia, coronary heart disease and chronic graft failure in kidney transplant recipients. Clin Nephrol 1999;51:290-5.

179. Dimény E, Wahlberg J, Lithell H and Fellström B. Hyperlipidaemia in renal transplantation risk factor for long-term graft outcome. Eur J Clin Invest 1995;25:574-83.

180. Roodnat JI, Mulder PGH, Zietse R, Rischen-Vos J, Riemsdijk IC van, IJzermans JNM and Weimar W. Cholesterol as an independent predictor of outcome after renal transplantation. Transplantation 2000;69:1704-10. 
181. Miles AMV, Sumrani N, Horowitz R, Homel P, Maursky V, Markell MS, Distant DA, Hong JH, Sommer BG and Friedman EA. Diabetes mellitus after renal transplantation. As deleterious as non-transplant-associated diabetes? Transplantation 1998;65:380-4.

182. Kooman JP, Christiaans MHL, Boots JMM, van der Sande FM, Leunissen KML, van Hooff JP. A comparison between office and ambulatory blood pressure measurements in renal transplant patients with chronic transplant nephropathy. Am J Kidney Dis 2001;37:1170-6.

183. Gjertson DW, Cecka JM and Terasaki PI. The relative effects of FK506 and cyclosporine on short- and long-term kidney graft survival. Transplantation 1995;60:1384-8.

184. Schleibner S, Krauss M, Wagner K, Erhard J, Christiaans M, Hooff J van, Buist L and Mayer D. FK 506 versus cyclosporin in the prevention of renal allograft rejection - European pilot study: six week results. Transpl Int 1995;8:86-90.

185. Niese D and on behalf of the International Sandimmun Neoral Study Group. A double-blind randomized study of Sandimmun Neoral versus Sandimmun in new renal transplant recipients: results after twelve months. Transplant Proc 1995;27:1849-56.

186. Keown P and Niese D on behalf of the International Sandimmun Neoral Study Group. Cyclosporine microemulsion increases drug exposure and reduces acute rejection without incremental toxicity in de novo renal transplantation. Kidney Int 1998;54:938-44.

187. Pollard SG, Lear PA, Ready AR, Moore RH and Johnson RWG on behalf of the UK Neoral Renal Study Group. Comparison of microemulsion and conventional formulations of cyclosporin A in preventing acute rejection in de novo kidney transplant patients. Transplantation 1999;68:1325-31.

188. Morris-Stiff G, Ostrowski K, Balaji V, Moore R, Darby C, Lord R and Jurewicz WA. Prospective randomized study comparing tacrolimus (Prograf) and cyclosporine (Neoral) as primary immunosuppression in cadaveric renal transplants at a single institution: interim report of the first 80 cases. Transpl Int 1998;11(S1):S334-6.

189. Undre NA, Hooff J van, Christiaans M, Vanrenterghem Y, Donck J, Heeman U, Kohnle M, Zanker W, Morales JM, Andrés A, Schäfer A and Stevenson P. Low systemic exposure to tacrolimus correlates with acute rejection. Transplant Proc 1999;31:296-8.

190. Ratcliffe PJ, Dudley CRK, Higgins RM, Firth JD, Smith B and Morris PJ. Randomised controlled trial of steroid withdrawal in renal transplant recipients receiving triple immunosuppression. Lancet 1996;348:643-8.

191. Schulak JA, Mayes JT, Moritz CE and Hricik DE. A prospective randomized trial of prednisone versus no prednisone maintenance therapy in cyclosporine-treated and azathioprine-treated renal transplant patients. Transplantation 1990;49:327-32.

192. Shapiro R, Jordan ML, Scantlebury VP, Vivas C, Fung JJ, McCauley J, Randhawa P, Demetris AJ, Irish W, Jain A, Mitchell S, Hakala TR, Simmons RL and Starzl TE. A prospective, randomized trial of FK 506/prednisone vs FK 506/azathioprine/prednisone in renal transplant patients. Transplant Proc 1995;27:814-7. 


\section{Chapter 2}

\section{Single center experience with tacrolimus versus cyclosporin-Neoral in renal transplant recipients}

JMM Boots, EM van Duijnhoven, MHL Christiaans, FHM Nieman, R-J van Suylen, JP van Hooff

Transplant International 2001;14(6):370-383 


\section{Abstract}

Tacrolimus has proven to be superior to cyclosporin-Sandimmune with regard to the prevention of acute rejections, but data comparing tacrolimus with Neoral are scarce.

Studied were 128 consecutive renal transplant recipients. They were treated with Neoral-based $(n=74)$ or tacrolimus-based $(n=54)$ immunosuppressive regimens. Survival analyses (Cox regression analysis) were performed on an intention-totreat basis. Renal function and cardiovascular risk profile were analyzed with a repeated measures ANOVA up to 12 months post-transplantation.

Immunological features were less favourable in the tacrolimus group. Two-year patient and graft survival were comparable. Acute rejection-free survival was $82 \%$ in the tacrolimus group versus $40 \%$ in the Neoral group $(P<0.0001)$. The severity of the rejections (1997 Banff classification) was comparable $(P=0.43)$. Immunological graft loss $(3.7 \%$ versus $12.2 \%, P=0.02)$ and conversion because of rejection $(0 \%$ versus $28.4 \%, P<0.001)$ were less in the tacrolimus group. A higher proportion $(68.5 \%$ versus $14.9 \%, P<0.001)$ was successfully put on monotherapy. Creatinine clearance, proteinuria, and fractional uric acid clearance were similar. In the tacrolimus group mean blood pressure was comparable, but patients needed less anti-hypertensive drugs $(P<0.001)$ and, even with fewer patients on lipidlowering drugs, total cholesterol was lower (5.2 versus $6.0 \mathrm{mmol} / 1, P=0.003$ ). Treatment for post-transplant diabetes mellitus was $18.5 \%$ versus $10.8 \%(P=0.22)$. In both groups, most patients could be withdrawn from anti-diabetic medication. This study indicates that tacrolimus is superior to cyclosporin-Neoral in preventing acute rejection with comparable patient and graft survival rates. Because of a lower need for treatment of hypertension and hypercholesterolemia, the cardiovascular risk profile is more favourable. A considerable proportion of patients can be successfully weaned from co-medication and treated with tacrolimus monotherapy. 


\section{Introduction}

Cyclosporin A (Sandimmune ${ }^{\circledR}$, Sandoz/Novartis, Switzerland) was introduced in renal transplantation in the $1970 \mathrm{~s}^{1}$. The major advantage was a reduction in the number of acute rejections and improvement in the 1 -year graft survival ${ }^{2-4}$. Its impact on medium- and long-term graft survival has not been firmly established ${ }^{5}$. Chronic allograft rejection is a major cause of graft loss. Prior acute rejections, especially steroid-resistant and recurrent rejections, appear to be an important contributory factor ${ }^{6-14}$. In 1995, in the Netherlands, cyclosporin-Sandimmune ${ }^{\circledR}$ was replaced by cyclosporin-Neoral ${ }^{\circledR}$ (Novartis, Switzerland), a new microemulsion formulation. This formulation has a more rapid and consistent absorption, leading to lower intra-patient variability ${ }^{15}$. In controlled trials, Neoral has proven to be superior to Sandimmune in the prevention of acute rejection ${ }^{16-18}$. Randomized multi-center trials have shown the superiority of tacrolimus (Prograf ${ }^{\circledR}$, Fujisawa, Japan)-based immunosuppressive regimens to cyclosporin (Sandimmune ${ }^{\circledR}$ ) with regard to the prevention of acute and steroid-resistant rejection ${ }^{19-23}$. Recently, a meta-analysis confirmed the results of the separate trials $^{24}$. Data comparing tacrolimus with Neoral in renal transplantation are scarce. One retrospective study showed a lower incidence of acute rejections with tacrolimus versus Neoral ${ }^{25}$. Another group published two interim reports of a prospective trial 26,27 . In the first report, with relatively low initial tacrolimus target levels, a similar incidence of acute rejection with tacrolimus versus Neoral was reported $^{27}$. In the second report, tacrolimus was superior to Neoral26.

With regard to side effects, tacrolimus administration resulted in advantages in cardiovascular risk profile. The incidence of hyperlipidemia was lower $19,21,26,28,29$. Although the incidence of hypertension in the main prospective trials was comparable ${ }^{20,21}$, there are now also indications, that less anti-hypertensive drugs are needed with tacrolimus ${ }^{19,26,30-32}$. Another advantage was a steroid-sparing effect ${ }^{19,32}$. Furthermore, the incidence of gingival hyperplasia and hirsutism, seen with cyclosporin, was remarkably lower in tacrolimus treated patients ${ }^{20,21}$. An important disadvantage of tacrolimus was a higher incidence of post-transplant diabetes mellitus ${ }^{20,21,23}$. Also tremor, pruritus, and alopecia were more frequently observed $^{20,21}$.

Because data comparing tacrolimus with Neoral are limited and prospective data have only been published by one group, we analyzed all of our patients treated with standard immunosuppressive therapy based on cyclosporin-Neoral and compared them with patients treated with tacrolimus-based immunosuppressive regimens in the same time period. 


\section{Materials and methods}

All patients receiving consecutive renal transplants between July 1995 and October 1997 were included in the study. Combined kidney and pancreas transplant recipients were excluded. Follow-up data were collected until May 1 1999, death, or graft failure.

\section{Immunosuppression}

\section{Neoral}

Standard initial immunosuppression from July 1995 until mid-September 1997 consisted of Neoral and steroids (prednisolone 10-20 mg/day).

Neoral was initially administered intravenously before surgery in a starting dose of $4 \mathrm{mg} / \mathrm{kg}$ per 24 hours and switched to oral intake ( $4 \mathrm{mg} / \mathrm{kg}$ b.i.d.) within 48 hours after surgery. In the case of an uneventful course, prednisolone was tapered to 7.5 $\mathrm{mg}$ at month 1 and to $5 \mathrm{mg}$ at month 3 . In recipients without rejection, prednisolone was further tapered to $0 \mathrm{mg}$ in the months that followed.

Recipients with panel-reactive antibodies (PRA) greater than 85\% in either peak or current serum and recipients with immunological failure of a previous graft received also azathioprine, $1 \mathrm{mg} / \mathrm{kg}(\mathrm{n}=4)$. Tapering prednisolone in these patients was restricted to $5 \mathrm{mg}$. All except one recipient with living related grafts also received azathioprine $(\mathrm{n}=11)$.

Whole blood trough cyclosporin target levels (radioimmunoassay (RIA), Syva, Dade-Behring, USA) were initially $0.15-0.20 \mathrm{mg} / 1$ in recipients receiving Neoral and steroids and $0.10-0.15 \mathrm{mg} / \mathrm{l}$ in recipients receiving Neoral, steroids, and azathioprine. The dose was gradually adjusted to target levels after 3 months of $0.10-0.15 \mathrm{mg} / \mathrm{l}$ in recipients receiving Neoral and steroids, and $0.05-0.10 \mathrm{mg} / \mathrm{l}$ in recipients receiving triple therapy with the addition of azathioprine.

Forty of the Neoral-treated patients $(54.1 \%)$ participated in a placebo-controlled Dutch multicenter trial, in which was investigated, whether the addition of isradipine (Lomir ${ }^{\circledR}$, Novartis, Switzerland) to standard immunosuppressive therapy would result in improvement in renal transplantation.

\section{Tacrolimus}

During the same period, indications for tacrolimus were: participation in a multicenter trial (FG-220) ( $n=25)$, compassionate use $(n=23)$, or standard therapy $(n=6)$, after the introduction of tacrolimus in the Netherlands in mid-September 1997.

Tacrolimus was only administered orally, with starting doses of $0.10-0.15 \mathrm{mg} / \mathrm{kg}$ twice daily, within 12 hours after transplantation. Whole blood tacrolimus trough target levels (micro-particle immunoassay, IMx, Abbot Laboratories, Abbott Park, 
IL, USA) were $10-20 \mathrm{ng} / \mathrm{ml}$ in the first 3 months post-transplantation. The dose of tacrolimus was gradually adjusted to target levels of $5-7 \mathrm{ng} / \mathrm{ml}$ after 6 months. All patients received steroids (10-20 mg prednisolone). In the case of an uneventful course, prednisolone was tapered to $7.5 \mathrm{mg}$ at month 1 and further to $5 \mathrm{mg}$ at month 3. In recipients without rejection, prednisolone was gradually withdrawn in the months that followed.

The FG-220 multi-center trial compared the value of adding mycophenolatemofetil (MMF, Cellcept ${ }^{\circledR}$, Roche, Switzerland) in doses of 1 and $2 \mathrm{~g} /$ day. All adults were eligible for this trial, with the exception of those with present or previous malignancies, liver disease, or recent infection. Twenty-five recipients participated in this trial. Seven recipients received $2 \mathrm{~g} \mathrm{MMF} /$ day, eight $1 \mathrm{~g}$, and ten received only tacrolimus and steroids.

Twenty-three recipients received tacrolimus on a compassionate use basis for the following indications:

1. highly immunized patients with contra-indications to azathioprine or previous use of recombinant anti-thymocyte globulin (r-ATG) $(n=7)$;

2. early graft loss of a previous transplant because of acute rejection or primary non-function with cyclosporin $(\mathrm{n}=2)$;

3. intolerance to, or severe side effects from, cyclosporin hemolytic uremic syndrome (HUS) ( $\mathrm{n}=3$ );

4. high-risk transplants due to living unrelated donors $(n=7)$, high urgency, or poor HLA-match $(\mathrm{n}=2)$;

5. prior use of tacrolimus due to participation in a previous trial $(n=2)$.

Two patients of subgroup 4 received also MMF $1 \mathrm{~g} /$ day: one living unrelated graft and one high urgency patient with a poor HLA-match.

Within the six patients who received tacrolimus as standard therapy, one patient with a living related donor received MMF $0.5 \mathrm{~g} /$ day.

Azathioprine $1 \mathrm{mg} / \mathrm{kg}$ was added to nine patients: five with a living unrelated graft, one with a living related graft, two re-transplants (HUS), and one highly immunized recipient.

\section{Conversion}

In the case of severe side effects, persistent or steroid-resistant rejections, patients could be converted to the opposite drug.

\section{Clinical outcome parameters}

Primary endpoints were patient survival, graft survival, acute rejection-free survival, and the severity of the rejection episodes.

Graft loss was defined as return to dialysis, independent of its cause, or death with a functioning graft. 
Acute rejection was defined on clinical criteria in the first six months posttransplantation and confirmed with an ultrasound-guided needle core biopsy. Episodes, in which treatment for an acute rejection was given and no biopsy could be performed due to contra-indications, were considered to be an acute rejection. There was clinical suspicion of rejection in the case of an unexplained rise or insufficient decrease in serum creatinine, with or without other signs, such as fever, tenderness of the graft, decreased renal perfusion on nuclear scan, or hypertension. Contra-indications for biopsy were bleeding disorders, uncontrolled severe hypertension, or neonatal kidneys. In the case of delayed graft function (need for dialysis in the first week after transplantation), a protocol biopsy was performed at week 1 to rule out rejection as the cause of non-functioning of the graft. Rejection treatment consisted of a course of three doses of 500-1000 mg methylprednisolone. In the case of steroid-resistant or vascular rejection, a ten-day course of r-ATG (RIVM, Bilthoven, the Netherlands) was used.

A pathologist classified the biopsies in a blinded fashion according to the 1997 revised Banff classification ${ }^{33}$.

Secondary endpoints were the course of the renal function and the cardiovascular risk profile, as they may be drug related.

Renal function parameters were creatinine clearance, calculated with the Cockcroft Gault formula and expressed per $1.73 \mathrm{~m}^{2}$ body surface area ${ }^{34}$, proteinuria, expressed as g protein $/ \mathrm{mol}$ creatinine excretion ${ }^{35}$, and fractional uric acid clearance $=($ urinary uric acid $\mathrm{x}$ serum creatinine $/$ urinary creatinine $\mathrm{x}$ serum uric acid) $\times 100 \%$ ).

The cardiovascular risk profile was assessed by measuring systolic and diastolic blood pressure, total cholesterol, and incidence of diabetes. Blood pressure was measured in the sitting position during outpatient clinic visits, according to the method of Riva Rocci. The mean blood pressure (defined as diastolic blood pressure + (systolic - diastolic blood pressure)/3) was calculated. The use of drugs for hypertension or hypercholesterolemia was established from the medical record. Diuretics were not included because they could have been used for reasons other than hypertension (e.g., edema or congestive heart failure). We classified patients for post-transplant diabetes if oral anti-diabetic drugs or insulin was used at any time after transplantation and if no diagnosis of diabetes had been established before the transplantation.

\section{Statistical analysis}

For comparison of baseline characteristics we used Student's t-test or, in the case of a non-parametrical distribution, the Mann-Whitney test. For comparison of categorical variables, Pearson's chi-square test was used.

To estimate patient survival, graft survival and acute rejection-free survival we used Cox's proportional hazards regression analysis. For the estimation of patient survival and graft survival, we included the following variables: use of tacrolimus 
or Neoral, use of azathioprine or MMF, HLA-A -, HLA-B - and HLA-DR mismatch $(0$ versus $\geq 1)$, transplant type (postmortem versus living), transplant number (1 versus >1), preservation solution [University of Wisconsin (UW) versus histidine-tryptophan-ketoglutarate (HTK) versus machine preservation], both warm (minutes) - and cold (hours) ischemia times, peak PRA level (\%), donor age (years), donor gender (male versus female), recipient age (years), recipient gender (male versus female), original renal disease [glomerulonephritis versus pyelonephritis versus nephrosclerosis versus diabetes mellitus type 1 (DM) versus DM type 2 versus other], and the presence of a rejection in the first 6 months (yes versus no). For the estimation of the acute rejection-free survival donor gender, both warm and cold ischemia times, transplant type, preservation solution, and the original renal disease were not suspected as being risk factors and were, thus, not analyzed. A basic model was tested for, which had to include only direct odds ratio effects that were statistically significant. When two factors were highly associated, the one that was more strongly related to the dependent variable was chosen. The possibility of interactions between factors in the basic model (different relative effects within different subgroups) was not taken into account, since too many additional terms would have had to have been included to test for such effects. These analyses were made on an intention-to-treat basis.

Changes in clinical outcome variables were assessed by a repeated measures analysis of variance (ANOVA). In the case of a non-parametrical distribution, a $\log$ transformation was performed. Recipients were included until graft loss, conversion to the opposite drug, or the moment they were lost to follow-up. Measurements were made at 3, 6, 9, and 12 months post-transplantation. The orthogonal polynomial contrasts were used to calculate linear, quadratic, etc. effects separately here within. The other factor was between patients: tacrolimus versus Neoral. The time-by-group interaction indicates differences in polynomial time trends. Overall effects are calculated in $\mathrm{F}$ ratios with degrees of freedom (df) and $\mathrm{p}$-values for non-significance. When Mauchly's W-test was lower than $P<0.05$, sphericity of the variance-covariance matrix of repeated measures was assumed to be violated. Univariate tests of $\mathrm{F}$ ratios were then conservatively corrected using an epsilon value suggested by Greenhouse and Geisser to correct degrees of freedom. Specific contrasts were only interrupted when overall effects compromising them showed statistical significance.

A $P$-value below 0.05 was considered to be statistically significant.

\section{Results}

In the study period 128 consecutive renal transplantations were performed. Seventy-four recipients received primarily Neoral and 54 received tacrolimus. 
Immunological features were less favourable in the tacrolimus group (Table 2.1). More patients underwent a re-transplantation $(31.5 \%$ versus $10.8 \%, P=0.004$, chisquare test). More patients were immunized or highly immunized (PRA 5-85\%: $27.8 \%$ versus $12.2 \%$ and PRA $\geq 85 \%$ : $9.3 \%$ versus $4.1 \%, P=0.03$, chi-square test). In addition, more patients received a kidney from living-unrelated donors $(13 \%$ versus $0 \%$ ). Other donor and transplant characteristics were comparable between the two groups (Table 2.1). Causes of death of the donors and type of preservation did not differ significantly either between the two groups. Differences did exist in the diagnosis of the renal insufficiency $(P=0.006$, chisquare test): more patients with chronic glomerulonephritis (35.2\% versus $14.9 \%)$ and nephrosclerosis (18.5\% versus $8.1 \%$ ) were included in the tacrolimus group; more patients with polycystic kidney disease $(21.6 \%$ versus $9.3 \%)$ and miscellaneous causes $(36.5 \%$ versus $16.7 \%$ ) were included in the Neoral group. Diabetic nephropathy was the diagnosis in $3.7 \%$ of the tacrolimus group versus $6.8 \%$ of the Neoral group.

MMF was included in the primary immunosuppression in 18 patients: Fifteen participated in a tacrolimus-based trial; three received MMF on a compassionate use basis. The use of azathioprine was not significantly different in the two groups $(16.7 \%$ in the tacrolimus group versus $20.3 \%$ in the Neoral group, $P=0.61$, chisquare test).

\section{Primary endpoints}

\section{Patient survival}

In the tacrolimus group, four patients died with a functioning graft $(7.4 \%)$ versus three $(4.1 \%)$ in the Neoral group $(P=0.39$, chi-square test). In both groups two patients died of infectious complications and one patient of severe hemorrhage. The other patient in the tacrolimus group died of a myocardial infarction.

In the Cox-regression analysis, recipient age, donor age, and peak PRA level were significant risk factors contributing to patient survival (Table 2.2). The type of immunosuppression (Neoral-based versus tacrolimus-based) did not contribute significantly to patient survival. The odds ratio for Neoral versus tacrolimus was 0.85 (95\% confidence interval: $0.2-4.5 ; P=0.85)$. The predicted two-year survival was $98 \%$ in the tacrolimus group and $99 \%$ in the Neoral group. 
Table 2.1: Base-line characteristics

\begin{tabular}{|c|c|c|c|c|}
\hline & & $\begin{array}{c}\text { CsA-Neoral } \\
\mathrm{n}=74\end{array}$ & $\begin{array}{c}\text { Tacrolimus } \\
\mathrm{n}=54\end{array}$ & $P$-value \\
\hline \multicolumn{2}{|l|}{ Age (yr) } & $48.5(17.5-73.4)$ & $51.2(20.6-67.5)$ & $0.25^{\mathrm{a}}$ \\
\hline \multicolumn{2}{|c|}{ Gender male/female (nr) } & $47(63.5) / 27(36.5)$ & $36(66.7) / 18(33.3)$ & $0.71^{\mathrm{b}}$ \\
\hline \multicolumn{2}{|c|}{ Mean arterial pressure $(\mathrm{mmHg})$} & $103.5(70-140)$ & $104.7(73-137)$ & $0.62^{\mathrm{a}}$ \\
\hline \multicolumn{2}{|l|}{ Hypertension (nr) } & $66(89.2)$ & $48(88.9)$ & $0.96^{\mathrm{b}}$ \\
\hline \multicolumn{2}{|l|}{ Anti-hypertensive drugs } & $1.46(0-4)$ & $1.48(0-5)$ & $0.32^{\mathrm{b}}$ \\
\hline \multicolumn{2}{|l|}{ Cholesterol (mmol/l) } & $5.5(3.1-8.9)$ & $5.3(2.5-7.1)$ & $0.54^{a}$ \\
\hline \multicolumn{2}{|c|}{ On lipid-lowering drugs (nr) } & $9(12.2)$ & $6(11.1)$ & $0.86^{\mathrm{b}}$ \\
\hline \multicolumn{2}{|c|}{ Diabetes mellitus (nr) } & $5(6.8)$ & $2(3.7)$ & $0.58^{\mathrm{b}}$ \\
\hline \multicolumn{2}{|c|}{ HLA-A $\quad(\geq 1$ mismatch) (nr) } & $49(66.2)$ & $40(74.1)$ & $0.34^{b}$ \\
\hline \multicolumn{2}{|c|}{ HLA-B $\quad(\geq 1$ mismatch) (nr) } & $49(66.2)$ & $43(79.6)$ & $0.10^{\mathrm{b}}$ \\
\hline \multicolumn{2}{|c|}{ HLA-DR ( $\geq 1$ mismatch) (nr) } & $41(55.4)$ & $36(66.7)$ & $0.20^{\mathrm{b}}$ \\
\hline \multicolumn{2}{|c|}{ Transplant number (nr) 1} & $66(89.2)$ & $37(68.5)$ & $0.004^{\mathrm{b}}$ \\
\hline \multirow{5}{*}{ PRA peak serum (nr) } & $>1$ & $8(10.8)$ & $17(31.5)$ & \\
\hline & $\leq 5 \%$ & $60(81.6)$ & $33(61.1)$ & $0.03^{\mathrm{b}}$ \\
\hline & $5-85 \%$ & $9(12.2)$ & $15(27.8)$ & \\
\hline & $\geq 85 \%$ & $3(4.1)$ & $5(9.3)$ & \\
\hline & missing & $2(2.7)$ & $1(1.9)$ & \\
\hline \multicolumn{2}{|l|}{ Donor age (yr) } & $44.3(4.4-72.5)$ & $46.0(4.8-69.6)$ & $0.58^{a}$ \\
\hline \multicolumn{2}{|c|}{ Donor gender male/female (nr) } & $42(56.8) / 32(43.2)$ & $27(50.0) / 27(50.0)$ & $0.45^{\mathrm{b}}$ \\
\hline \multicolumn{2}{|c|}{ Donor type (nr) } & & & $0.004^{b}$ \\
\hline \multicolumn{2}{|c|}{ Post mortal HB } & $46(62.2)$ & $30(55.6)$ & \\
\hline \multicolumn{2}{|c|}{ Post mortal non-HB } & $16(21.6)$ & $14(25.9)$ & \\
\hline \multicolumn{2}{|c|}{ Living related } & $12(16.2)$ & $3(5.6)$ & \\
\hline \multicolumn{2}{|l|}{ Living unrelated } & $0(0.0)$ & $7(13.0)$ & \\
\hline \multicolumn{2}{|c|}{ Cold ischemia time $(\mathrm{hr})^{*}$} & $27.6(16-43)$ & $25.3(8-50)$ & $0.12^{\mathrm{a}}$ \\
\hline \multicolumn{2}{|c|}{ Warm ischemia time 1 (min) } & $14.8(0-192)$ & $17.3(0-148)$ & $0.44^{c}$ \\
\hline \multicolumn{2}{|c|}{ Warm ischemia time 2 (min) } & $34.3(18-65)$ & $35.6(23-80)$ & $0.47 \mathrm{c}$ \\
\hline
\end{tabular}

Values are expressed as mean (range) or as numbers $(\%) .{ }^{*}=$ living donor excluded; PRA=panel reactive HLA-antibodies; HB=heart-beating; ${ }^{a}=$ Student's t-test; b=Pearson's $\chi^{2}$-test; c=MannWhitney test

\section{Graft survival}

Graft loss and graft loss, censored for death, were comparable between both groups (tacrolimus versus Neoral: $24.1 \%$ versus $20.2 \%, P=0.61$, and $16.7 \%$ versus $16.2 \%, P=0.95$, respectively, chi-square test). When focusing on graft failure due to immunological causes, significantly fewer failures were observed in the tacrolimus group $(3.7 \%$ versus $12.2 \%, P=0.02$, chi-square test). 
In the Cox-regression analysis, the presence of an HLA-DR mismatch, donor age, cold ischemia time, and second warm ischemia time were significant risk factors contributing to graft survival (Table 2.2). The type of immunosuppression (Neoral-based versus tacrolimus-based) did not contribute significantly to graft survival. The odds ratio for Neoral versus tacrolimus was 0.82 (95\% confidence interval: $0.4-1.8 ; P=0.61)$. The predicted two-year graft survival was $84 \%$ in the tacrolimus group and $87 \%$ in the Neoral group (Figure 2.1).

In the Cox-regression analysis of graft survival, censored for death, only the presence of an HLA-DR mismatch contributed significantly to graft failure: odds ratio 5.1 (95\% confidence interval: $1.5-17.4 ; P=0.002)$. Again, the type of immunosuppression did not contribute significantly to the graft failure. The odds ratio for Neoral versus tacrolimus was $0.95(0.4-2.2 ; P=0.90)$. The predicted twoyear graft survival, censored for death, was $87 \%$ in both groups.

Table 2.2: Cox's proportional hazards regression analysis

\begin{tabular}{lcccc}
\hline Risk factor & OR & $\mathbf{9 5} \% \mathbf{C I}$ & df & $\boldsymbol{P}$-value \\
\hline Patient Survival & & & & \\
Age recipient (/year) & 1.14 & $1.01-1.29$ & 1 & 0.006 \\
Donor age (/year) & 1.06 & $0.99-1.13$ & 1 & 0.05 \\
Peak PRA (/\%) & 1.03 & $1.01-1.06$ & 1 & 0.006 \\
Neoral vs. tacrolimus & 0.85 & $0.16-4.5$ & 1 & 0.85 \\
Graft survival & & & & \\
HLA-DR $(\geq 1$ mismatch vs. 0) & 2.9 & $1.1-7.4$ & 1 & 0.01 \\
Donor age (/year) & 1.03 & $1.00-1.06$ & 1 & 0.01 \\
WIT-2 (/min) & 1.04 & $1.01-1.08$ & 1 & 0.03 \\
CIT (/hour) & 1.05 & $1.01-1.10$ & 1 & 0.01 \\
Neoral vs. tacrolimus & 0.8 & $0.4-1.8$ & 1 & 0.61 \\
HLA-DR $(\geq 1$ mismatch vs. 0) & 2.5 & & & \\
Neoral v.s. tacrolimus & 4.6 & $2.4-4.5$ & 1 & 0.002 \\
Rejection-free survival & & & 1 & $<0.0001$ \\
\hline
\end{tabular}

PRA=panel reactive HLA-antibodies; OR=Odds ratio; WIT-2=second warm ischemia time; $\mathrm{CI}=$ confidence interval; $\mathrm{CIT}=$ cold ischemia time; $\mathrm{df}=$ degrees of freedom 


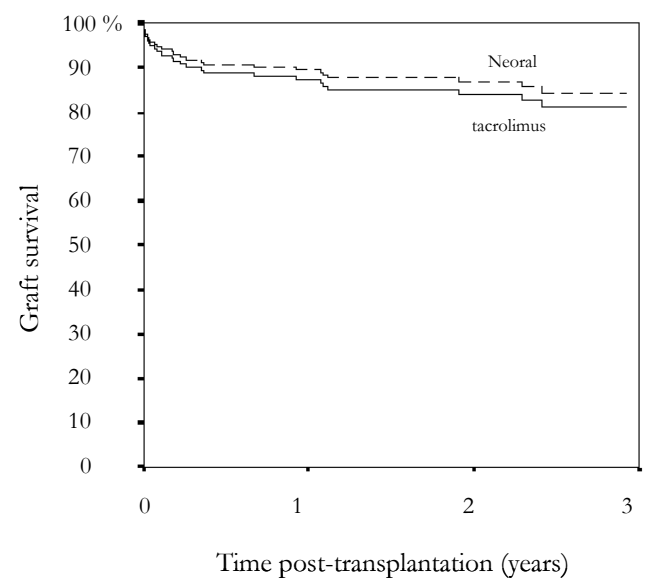

Figure 2.1 Predicted graft survival

Significant risk factors using Cox's proportional hazards regression analysis were the presence of a mismatch in HLA-DR, donor age, cold - and second warm ischemia time. The Odds ratio for Neoral versus tacrolimus was 0.8 ( $95 \%$ confidence interval: $0.4-1.8), P=0.61$.

\section{Acute rejection}

Fifty-four patients were treated for acute rejection. All but four of these episodes were biopsy confirmed. Three patients had no biopsy performed due to contraindications and in one patient there was insufficient biopsy material. Nevertheless, at a second episode of acute rejection, the diagnosis could be biopsy confirmed in two of these patients. The remaining two patients had a good clinical response on anti-rejection treatment with return of serum creatinine to baseline.

In the tacrolimus group, significantly fewer acute rejections were observed: $18.5 \%$ versus $59.5 \%$ for a primary acute rejection within the first six months $(P<0.001$, chi-square test) and $7.4 \%$ versus $33.8 \%$ for a second acute rejection $(P<0.001$, chisquare test). In the tacrolimus group, fewer patients were treated with r-ATG: $5.6 \%$ versus $13.5 \%$, but this did not reach statistical significance $(P=0.14$, chisquare test).

In the Cox-regression analysis, the use of Neoral and the presence of an HLA-DR mismatch were significant risk factors contributing to acute rejection-free survival (Table 2.2). The odds ratio for Neoral versus tacrolimus was 4.6 (95\% confidence interval: $2.3-9.2 ; P<0.0001)$. The predicted acute rejection-free survival was $82 \%$ in the tacrolimus group versus $40 \%$ in the Neoral group (Figure 2.2). 


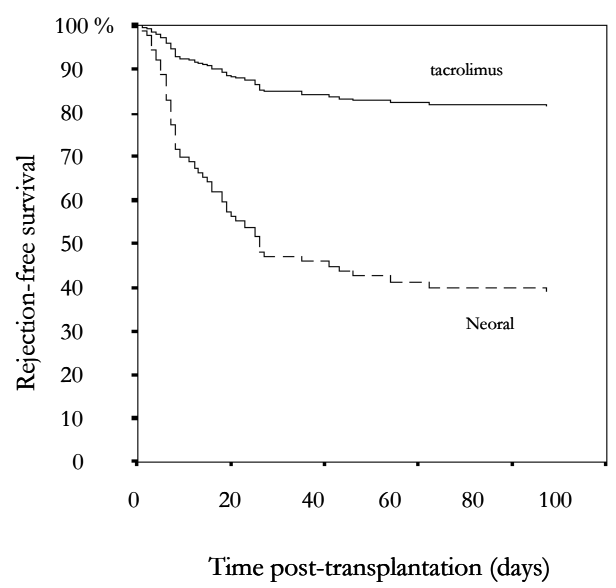

Figure 2.2 Predicted acute rejection-free survival

Significant risk factors using Cox's proportional hazards regression analysis were the presence of a mismatch in HLA-DR and the use of Neoral versus tacrolimus: Odds ratio 4.6 (95\% confidence interval: $2.3-9.2), P<0.0001$.

To test the possibility that the observed difference was due to the use of MMF in a subgroup of tacrolimus-treated patients, we analyzed the acute rejection-free survival in two ways: 1$)$ for patients with tacrolimus without MMF ( $\mathrm{n}=36)$ versus patients with tacrolimus and MMF ( $\mathrm{n}=18$ ), and 2) for patients with tacrolimus without MMF ( $\mathrm{n}=36)$ versus the Neoral group $(\mathrm{n}=74)$. The first analysis showed that the relative risk for rejection was not significantly higher in the patients without MMF (odds ratio 2.0, 95\% confidence interval: 0.4-9.7; $P=0.37$ ). The estimated acute rejection-free survival was $77 \%$ in the patients, treated without MMF versus $88 \%$ for the patients treated with tacrolimus and MMF (Figure 2.3). The second analysis showed that the increased risk for rejection in the Neoral group remained highly significant (odds ratio for Neoral 3.8, 95\% confidence interval: $1.8-8.1 ; P=0.0006)$. The predicted rejection-free survival was $78 \%$ in the tacrolimus group versus $40 \%$ in the Neoral group.

The relative distribution of the severity of the rejection episodes was not significantly different between the patients in the tacrolimus and the Neoral groups ( $P=0.43$ for the Banff score of the first rejection and $P=0.31$ for the maximum Banff score under the initial drug, chi-square test; Table 2.3). 


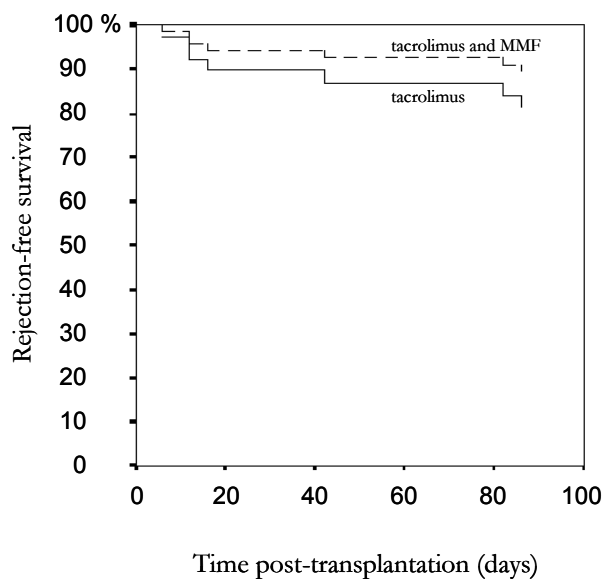

Figure 2.3 Predicted acute rejection-free survival

The predicted acute rejection-free survival using Cox's proportional hazards regression analysis between patients treated with tacrolimus without MMF ( $\mathrm{n}=36$ ) versus tacrolimus with MMF ( $\mathrm{n}=18)$ : Odds ratio 2.0 ( $95 \%$ confidence interval: 0.4-9.7), $P=0.37$

Table 2.3: Severity of the rejections after the 1997 Banff classification

\begin{tabular}{lccc}
\hline & $\begin{array}{c}\text { CsA-Neoral } \\
\mathbf{n}=\mathbf{4 4}\end{array}$ & $\begin{array}{c}\text { Tacrolimus } \\
\mathbf{n}=\mathbf{1 0}\end{array}$ & $\begin{array}{c}\boldsymbol{P} \text {-value } \\
\boldsymbol{\chi}^{2} \text {-test }\end{array}$ \\
\hline Banff score first rejection & & & 0.43 \\
Borderline & $3(6.8)$ & $3(30.0)$ & \\
1 A & $17(38.6)$ & $3(30.0)$ & \\
1 B & $13(29.5)$ & $2(20.0)$ & \\
2 A & $5(11.4)$ & $2(20.0)$ & \\
2 B & $1(2.3)$ & $0(0.0)$ & \\
3 & $0(0.0)$ & $0(0.0)$ & \\
Hyperacute & $1(2.3)$ & $0(0.0)$ & \\
No histology & $4(9.1)$ & $0(0.0)$ & \\
Maximum Banff score under initial drug & & & \\
Borderline & $2(4.5)$ & $2(20.0)$ & \\
1 A & $14(31.8)$ & $2(20.0)$ & \\
1 B & $16(36.4)$ & $3(30.0)$ & \\
2 A & $5(11.4)$ & $3(30.0)$ & \\
2 B & $4(9.1)$ & $0(0.0)$ & \\
3 & $0(0.0)$ & $0(0.0)$ & \\
Hyperacute & $1(2.3)$ & $0(0.0)$ & \\
No histology & $2(4.5)$ & $0(0.0)$ & \\
\hline
\end{tabular}


Significantly more patients were converted from Neoral to tacrolimus than from tacrolimus to Neoral $(31.1 \%$ versus $3.7 \%, P<0.001$, chi-square test). The main reason for conversion from Neoral to tacrolimus was an episode of acute rejection (21 out of 23 patients). No patients were converted from tacrolimus to Neoral because of rejection. Three patients were converted after the first episode of acute rejection, 14 after the second, and four after the third. Sixteen patients $(76.2 \%)$ were spared from further acute rejections. In the remaining five patients, a renewed acute rejection occurred 12, 29, 34, 180, and 1139 days after conversion. In two of these five patients, the rejection occurred after steroid withdrawal. In three of the five patients, the grafts were lost due to ongoing rejections.

In the tacrolimus group, significantly more patients could be withdrawn from steroids $(79.6 \%$ versus $41.9 \%, P<0.001$, chi-square test). At the time of steroid withdrawal, only four patients still used MMF $(9.3 \%$ of the patients in which the steroids were stopped). After stopping steroids three patients had an acute rejection 34, 126 and 482 days after steroid withdrawal. None were in the initial tacrolimus group.

At the end of the follow-up period, $70.4 \%$ of the patients in the tacrolimus group versus $27.0 \%$ in the Neoral group were actually on monotherapy $(P<0.001$, chisquare test). Censored for conversion, $68.5 \%$ in the tacrolimus group versus $14.9 \%$ in the Neoral group were on monotherapy $(P<0.001$, chi-square test).

\section{Secondary outcome parameters}

Because a considerable proportion of the patients in the Neoral group were converted to tacrolimus, only those patients, who were still on the primary drug at 12 months post-transplantation, and who had a complete follow-up were considered for the analysis of possibly drug-related clinical outcome parameters. In the tacrolimus group, one patient was converted, four patients had died, five grafts had failed, and two patients had an incomplete follow-up. In the Neoral group, 15 patients were converted, three patients had died, eight grafts had failed, and two patients had an incomplete follow-up. So, 88 patients were eligible, 42 in the tacrolimus group and 46 in the Neoral group.

\section{Renal function}

Creatinine clearance increased during the first year in both groups and stabilized between 9 and 12 months (Figure 2.4). At none of the time points were the differences between the two groups statistically significant $(P>0.30)$. 


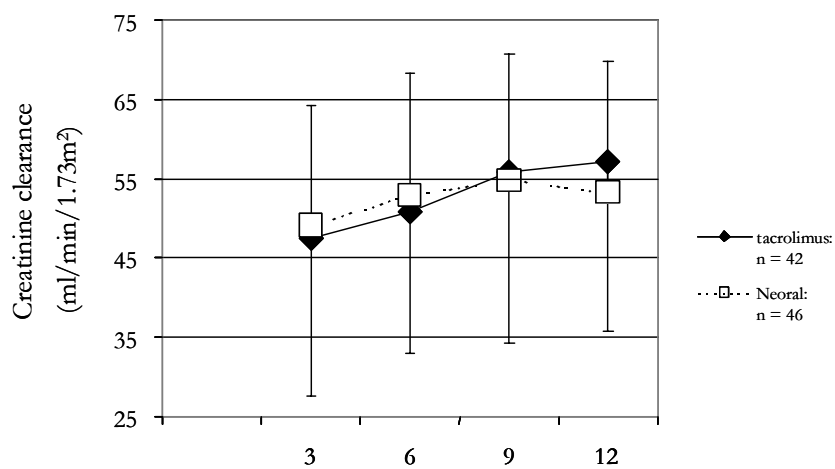

Time post-transplantation (months)

Figure 2.4 Creatinine clearance

In both groups creatinine clearance increased during the first year and stabilized between 9 and 12 months post-transplantation (ANOVA). Differences at each time point were not significant $(P>0.30$, Student's t-test).

Proteinuria was log-transformed because of a non-parametrical distribution. Logtransformed proteinuria showed a decrease in both groups up to nine months and an increase afterwards (Figure 2.5). Differences in proteinuria were not statistically significant between the two groups at any time point $(P>0.20)$.

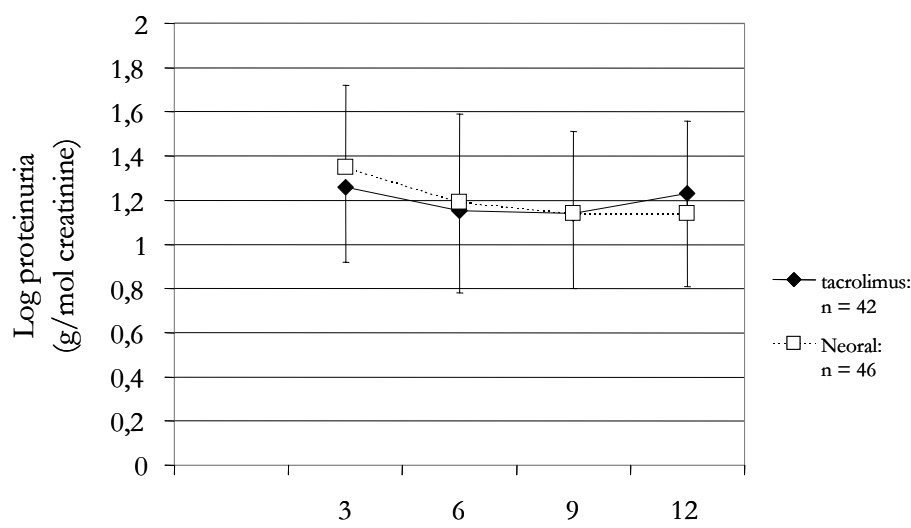

Time post-transplantation (months)

Figure 2.5 Proteinuria

Proteinuria was log transformed, because of a non-parametrical distribution. In both groups proteinuria decreased up to nine months post-transplantation and increased thereafter (ANOVA). Differences at each time point were not significant $(P>0.20$, Student's t-test). 
Fractional uric acid clearance decreased in a similar way in both groups (Greenhouse-Geisser corrected $\mathrm{F}$ ratio $=10.85$ by 2 and $206 \mathrm{df}, P<0.001$ ). There were no statistically significant differences between the two groups at any time point $(P>0.25)$.

\section{Cardiovascular risk profile}

Mean blood pressure showed no significant trend with time ( $F$ ratio $=1.06$ by 3 and $258 \mathrm{df}, P=0.37$; Figure 2.6). Differences between the two groups were not significant at any time point $(P>0.10)$.

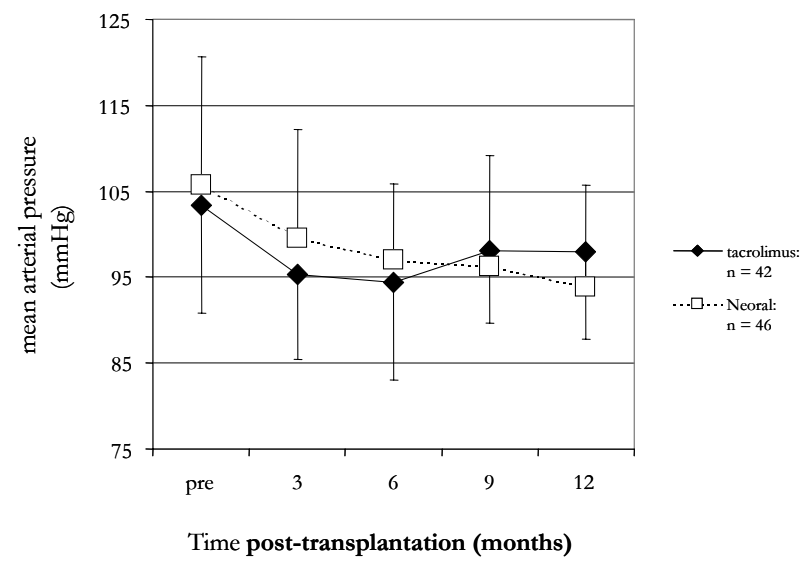

Figure 2.6 Mean arterial pressure

The mean arterial pressure showed no significant trend in time (ANOVA). Differences at each time point were not significant ( $\mathrm{P}>0.10$, Student's t-test).

Although blood pressure was comparable for both groups, patients in the Neoral group used significantly more anti-hypertensive drugs at 6,9 , and 12 months ( $P=0.09, P=0.008, P=0.001$, and $P<0.001$ at $3,6,9$, and 12 months, respectively, chi-square test; Figure 2.7). At 12 months post-transplantation, $21.4 \%$ of patients in the tacrolimus group versus $4.3 \%$ in the Neoral group were free of the use of anti-hypertensive drugs. Three drugs or more were used by $7.1 \%$ in the tacrolimus group versus $52.2 \%$ in the Neoral group.

Total cholesterol was significantly higher at all time points in the Neoral group $(P=0.04,0.03,<0.001$, and 0.003 at $3,6,9$, and 12 months after transplantation, respectively, Student's t-test; Figure 2.8). Notwithstanding the higher total cholesterol in the Neoral group, more patients in this group used lipid-lowering drugs, statistically significant up to six months $(P=0.004$ and $P=0.004$ at three and six months after transplantation, respectively, chi-square test; Figure 2.9). 


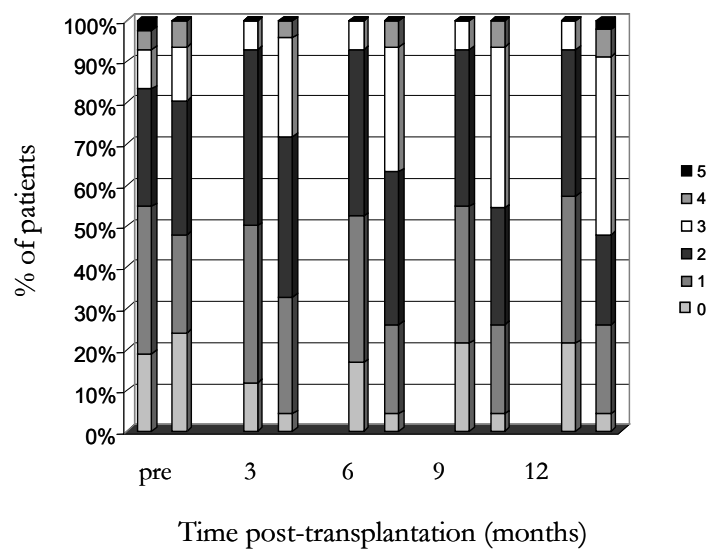

Figure 2.7 Anti-hypertensive medication

The right bar at each time point concerns the Neoral group ( $n=46)$; the left bar the tacrolimus group $(\mathrm{n}=42)$. The number of anti-hypertensive drugs, the patients are using is expressed in the bars. $P=0.09,0.008,0.001$, and $<0.001$ at $3,6,9$, and 12 months post-transplantation, respectively $\left(\chi^{2}\right.$-test). Diuretics are not included.

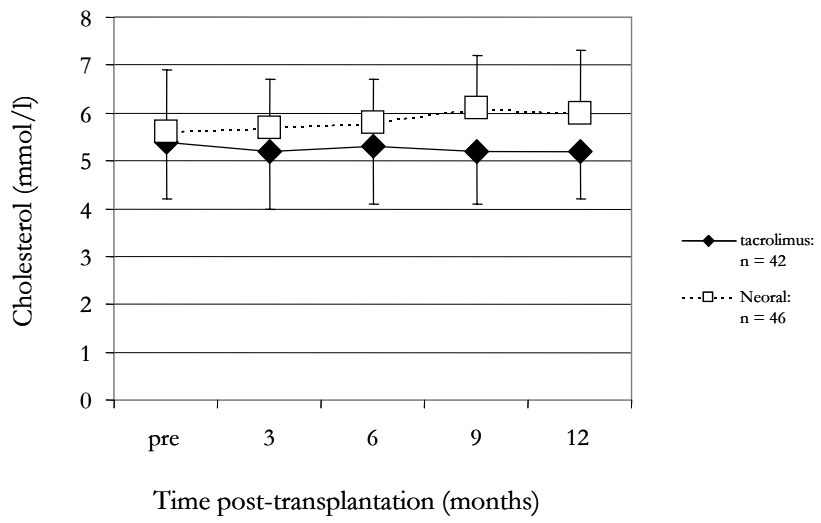

Figure 2.8 Total cholesterol

Total cholesterol showed no significant trend in time (ANOVA). Differences at each time point were significant: $P=0.04,0.03,<0.001$, and 0.003 at $3,6,9$, and 12 months post-transplantation, respectively (Student's t-test).

The overall incidence of post-transplant diabetes mellitus on an intention-to-treat analysis was higher in the tacrolimus group, but the difference was not statistically significant $(18.5 \%$ in the tacrolimus group versus $10.8 \%$ in the Neoral group, $P=0.22$, chi-square test). The need for treatment with insulin at any time after 
transplantation was nearly identical $(7.4 \%$ in the tacrolimus group versus $6.8 \%$ in the Neoral group, $P=0.89$, chi-square test). Of the patients needing treatment for diabetes, $70 \%$ in the tacrolimus group versus $50.0 \%$ in the Neoral group could be withdrawn from all anti-diabetic medication ( $P=0.51$, chi-square test).

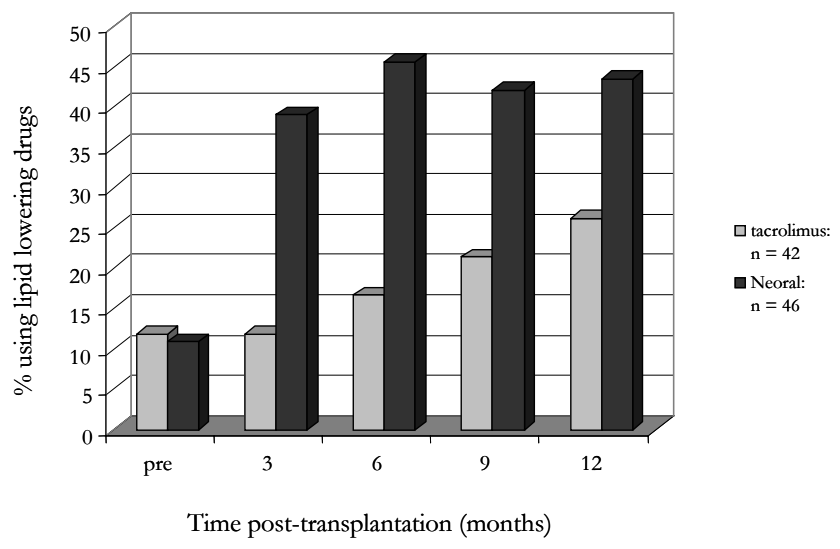

Figure 2.9 Use of lipid-lowering drugs

Differences between the tacrolimus and Neoral group were significant at 3 and 6 months post-transplantation: $P=0.004,0.004,0.09$, and 0.18 at $3,6,9$, and 12 months, respectively $\left(\chi^{2}\right.$-test).

\section{Conversion for side effects}

Besides conversion for acute rejection, two other patients were converted from Neoral to tacrolimus 1076 and 628 days after transplantation. For one patient it was because of gingival hyperplasia, and for the other it was because of osteoporosis and the need for steroid withdrawal despite a prior acute rejection. The further course of these two patients was uneventful.

Two patients were converted from tacrolimus to Neoral 505 and 25 days after transplantation because of side effects possibly related to tacrolimus: GuillainBarré syndrome and thrombotic thrombocytopenic purpura (TTP), respectively. After conversion no acute rejections were observed, but in the latter patient MMF was added, due to prior rejections. This graft failed due to recurrence of focal segmental glomerulosclerosis.

\section{Discussion}

In former trials, tacrolimus-based immunosuppression had been shown to be superior to cyclosporin (Sandimmune) with regard to the prevention of acute and steroid-resistant rejection ${ }^{19-23}$. Neoral, the new formulation of cyclosporin has 
proven to be superior to Sandimmune in the prevention of acute rejection ${ }^{16-18}$. Criticism arose, that it had not yet been proven, that tacrolimus was superior to Neoral. One group from Cardiff had published two interim reports of a prospective trial. In the first report tacrolimus and Neoral yielded similar results ${ }^{27}$. In the second report, tacrolimus was superior to Neoral ${ }^{26}$. In our single center study, we compared all consecutive renal transplant recipients treated with Neoral or tacrolimus-based immunosuppression during the same time period. Because tacrolimus was not yet registered in the Netherlands, for much of the period studied, a substantial number of the recipients received this drug on a compassionate use basis. This is reflected in a less favourable immunological profile in the tacrolimus group. More patients underwent a re-transplantation and had positive PRA-levels. Within the living donors, more grafts were received from living-unrelated donors. On the other hand a proportion of tacrolimus-treated patients received MMF. Patient and graft survival were comparable, but we observed a substantial lower rate of first and relapsing acute rejections and graft loss due to immunological causes in the tacrolimus group.

The predicted two-year patient survival rate was $98 \%$ in the tacrolimus group. This is comparable to the findings of other studies with tacrolimus ${ }^{19-21,23,25,32,36,37}$. In this study, a similar patient survival rate was observed between tacrolimus and Neoral-based immunosuppression. This is in concordance with the similar patient survival in studies comparing tacrolimus with cyclosporin ${ }^{19-21,23,32}$ and in a retrospective study comparing tacrolimus with $\mathrm{Neoral}^{25}$.

The two-year predicted graft survival rate with tacrolimus was $84 \%$. This is comparable to what has previously been reported with tacrolimus $5,19-21,23,26,32,36,37$. In previous studies comparing tacrolimus with cyclosporin, graft survival was comparable19-21,23,36. In our study, comparing tacrolimus with Neoral, graft survival was the same as that reported by Ghasemian et al. ${ }^{25}$. In contrast, in his interim report, Jurewicz reported a better graft survival with tacrolimus than with $\mathrm{Neoral}^{26}$.

Acute rejection-free survival was halved in the tacrolimus group. The rate of acute rejection in the tacrolimus group was $18.5 \%$, comparable to the $19.4 \% 22$ and $25 . \%^{20}$ in former European multi-center trials. By participating in those trials, our center acquired a lot of experience with tacrolimus. Such experience is important since, in a European multi-center trial ${ }^{38}$, the rate of acute rejection was lower in centers with experience with this drug 38,39 . In American and Japanese studies, the rate of rejection was higher $(30 \%-55 \%)^{21,32,36,37,40}$. Race (in this case, being black) and age (being young) are risk factors for rejection ${ }^{10,41-43}$. Differences in the patient groups in these factors could be an explanation for the higher rate of acute rejection with tacrolimus. 
In the tacrolimus group, 25 patients $(46.3 \%)$ participated in a trial, which could have led to bias in selection and management. In the trial-period nearly all consecutive transplant patients participated in that study. Five well-matched living related transplants and only one patient, who met the exclusion-criteria, were treated with Neoral. Besides, four living unrelated transplants in that period were treated with tacrolimus outside the trial. If any selection-bias had occurred, it would have been in favour of the Neoral group. In addition, an even larger proportion (54.1\%) participated in a trial in the Neoral group.

Also, outside the trial-period, the majority of the tacrolimus-treated patients belonged to a high-risk category. Thus, for the whole population studied, selection-bias was in favour of the Neoral group. This is reflected in a significantly higher proportion of re-transplants and higher PRA-levels in the tacrolimus group. Also, HLA-matching was better in the Neoral group.

Throughout the study period, all patients were treated by the same physicians. Pre- and post-transplant management and diagnosis and treatment of rejection were unchanged and were the same for Neoral-treated and tacrolimus-treated patients. Thus, better management of the patients, who participated in the trial, seems unlikely as an explanation for the lower incidence of acute rejection in the tacrolimus group.

The addition of MMF to the immunosuppressive regimen of a certain number of patients in the tacrolimus group does not explain fully the high rejection-free survival in this study because the rejection-free survival in the patients treated with tacrolimus without MMF was $77 \%$, compared to $88 \%$ in the patients treated with tacrolimus and MMF. Besides, after exclusion of the patients who received MMF, the difference with the Neoral group (78\% versus $40 \%)$ remained highly significant. Moreover the duration of the use of MMF was relatively short. Only one patient used MMF throughout the study period. In the remaining patients MMF was stopped after a mean of 106 days (range 6-254).

The rate of rejection in the Neoral group in this study resembles the rate found earlier in Sandimmune-treated recipients ${ }^{10,16,17,32}$. In recent studies, the rate of rejection with Neoral is around 40\%16-18,25-27. In some of these studies, the upper target trough level of cyclosporin was higher, with values up to $0.3-0.4 \mathrm{mg} / \mathrm{l}^{18,25}$. Higher dosing results in a lower rate of acute rejection ${ }^{10,44}$, but poses the risk of dose-dependant nephrotoxicity 45 and may even have a negative impact on graft survival $^{10}$. Also, in most studies, more patients received triple therapy with the addition of azathioprine or $\mathrm{MMF}^{16,17,25-27}$ and, when mentioned, the steroid dose was higher ${ }^{26,27}$. In one study, high-risk recipients were excluded ${ }^{18}$.

In our study, nearly all rejections were biopsy-proven and classification as borderline was scarce. Moreover, renal function improved after rejection treatment in the patients without biopsy. Thus, the high incidence of acute rejection cannot be the result of overtreatment of clinical suspicion of acute 
rejection. To test the possibility of underdosing of Neoral, blood levels at the time of the first rejection were analyzed. The mean cyclosporin trough level was $0.22 \mathrm{mg} / 1$ (SD 0.11). Only nine patients with a rejection (20.5\%) had a level below the lower target level. Thus, only in a minority might underdosing be the explanation for the rejection; it cannot account for the relatively high incidence of rejection in the Neoral-treated patients.

In the studies comparing the rejection rate in patients treated with tacrolimus versus Neoral, Ghasemian et al. ${ }^{25}$ and Jurewicz, in the second interim report of the Cardiff study group ${ }^{26}$, reported a comparable rate of acute rejection with tacrolimus and a twice as high incidence in the Neoral group. Yet, in the initial interim report of the Cardiff study group, Morris-Stiff et al. reported a substantially higher rate of acute rejection of $40 \%$ with tacrolimus ${ }^{27}$. This latter observation can probably be explained by their relatively low initial tacrolimus target levels of $5-15 \mathrm{ng} / \mathrm{ml}$. It is well proven that the risk of acute rejection is enhanced when initial target levels are below $10 \mathrm{ng} / \mathrm{ml}^{46}$.

An important risk factor for acute rejection is the degree of HLA-matching, especially for HLA-DR ${ }^{10,47}$. When corrected for this risk factor, the rate of acute rejection for the tacrolimus-treated patients, compared to the Neoral-treated patients, was still highly significant. From the reported data, differences in HLADR mismatches between this study and other studies could not be

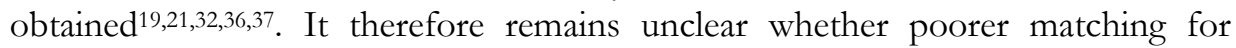
HLA-DR is an additional explanation for the higher rate of acute rejection with tacrolimus in those studies.

Acute rejection, and especially steroid-resistant rejection, is a risk factor for the development of chronic allograft rejection, a major cause of graft loss after one year ${ }^{6-14}$. In our study, no improvement in graft survival was found, but the lower incidence of acute rejections might have had an impact on improvement in longterm graft survival 5 .

When a rejection occurred, the relative severity was comparable between tacrolimus and Neoral. This means that tacrolimus equally reduces all kind of rejections and not only borderline and grade 1 . Our study is, to our knowledge, the first one that demonstrates the lower incidence of all grades of acute rejection with tacrolimus in relation to Neoral. In a previous multi-center study, the same result was found with Sandimmune ${ }^{20}$.

A unique property of tacrolimus is that, if irreversible rejections occur after conversion from cyclosporin to tacrolimus, the graft can be saved ${ }^{21,48-51}$. Similar observations after conversion from Neoral to tacrolimus are scarce 25,27 . In our 
study, $76.2 \%$ of the patients converted to tacrolimus for acute rejection with Neoral experienced no further rejections. Thus, the results obtained with Sandimmune could be confirmed.

Steroid-related side effects are a major cause of morbidity in renal transplant recipients ${ }^{52}$. Nearly $80 \%$ of the patients in the tacrolimus group could be withdrawn from steroids. None had a rejection after withdrawal, and only $9.3 \%$ of these patients used MMF at the time of steroid withdrawal. Other groups have also reported a reasonable number of patients without steroids, ranging from $35 \%$ to $55 \% 0^{32,36,40}$. Only one study reported a comparison with Sandimmune-treated patients, in which all Sandimmune-treated patients received some dose of steroids $^{32}$. We were able to withdraw steroids in $42 \%$ of the Neoral-treated patients, but that was significantly less than in the tacrolimus group. It was recently demonstrated that it is probably safe to withdraw steroids in cyclosporintreated, non-black patients when MMF is added ${ }^{53}$. In our study, $68.5 \%$ of the patients in the tacrolimus group were successfully put on monotherapy versus only $14.9 \%$ in the Neoral group. In our opinion, the safety of steroid withdrawal in patients without an acute rejection is another advantage of tacrolimus.

It has been reported that the need for treatment with r-ATG for steroid-resistant rejection was significantly lower with tacrolimus than with either Sandimmune ${ }^{20,21}$ or Neoral ${ }^{25}$. Although we also observed a lower need for r-ATG, the difference was not statistically significant, as in the study of Jurewicz ${ }^{26}$.

Both tacrolimus and cyclosporin are nephrotoxic drugs ${ }^{45}$. In our study, renal function in patients with a functioning graft 12 months after transplantation was comparable with tacrolimus and Neoral. This is in accordance with previous reports in which tacrolimus was compared to Sandimmune ${ }^{19-23,32}$ or Neoral25,27. In contrast, Jurewicz reported a lower serum creatinine in the tacrolimus group than in the Neoral group ${ }^{26}$. Data on differences in proteinuria between tacrolimus and Sandimmune or Neoral have not been published. We found a similar rate of proteinuria between tacrolimus and Neoral. Also, fractional uric acid clearance was comparable in our study. In another study, serum uric acid increased more over time with Neoral, but the difference with tacrolimus was not significant and could have been influenced by the higher serum creatinine in the Neoral group in that study ${ }^{26}$.

Both hypertension and hyperlipidemia have been associated with long-term graft outcome 54,55 . The targets for blood pressure and cholesterol were the same for both groups, whether or not the patients participated in a trial. Differences in management of these parameters cannot account for differences between the groups. The cardiovascular risk profile in our study was more favourable with 
tacrolimus than with Neoral. Although the mean arterial pressure was comparable, the need for anti-hypertensive drugs was lower in the tacrolimus group and more patients were without anti-hypertensive treatment. This is in accordance with previous reports in which tacrolimus was compared to both Sandimmune ${ }^{19,21,22,32}$ and Neoral ${ }^{26}$.

Total cholesterol was also lower in the tacrolimus group, even with fewer patients taking lipid-lowering drugs. Previous studies have shown similar results when comparing tacrolimus with Sandimmune ${ }^{19,21,32}$ or Neoral26,27. After steroid withdrawal, both hypertension and the lipid profile improve ${ }^{53}$. In our study, a considerable proportion of the tacrolimus-treated patients were not on steroids. This could have influenced the more favourable cardiovascular risk profile. However, the more favourable lipid profile persists ${ }^{21,28,29}$, and there are indications that less anti-hypertensive treatment is necessary with tacrolimus in studies with equal doses of steroids 30,31 . Thus, the results in our study can probably not be totally explained by the lower use of steroids.

In our study a considerable proportion of the patients in the Neoral group (25.4\% of the patients with one year follow-up) were converted to tacrolimus, in all cases because of acute rejection. Because we wanted to evaluate differences in drugrelated side effects between the two drugs and because we wanted to evaluate the development of the parameters in the time post-transplantation, analyses were made after censoring for conversion. This could have influenced the composition of the study groups. We re-analyzed the parameters on an intention-to-treat basis. After one year post-transplantation, the following parameters analyzed by Student's t-test were still comparable between the tacrolimus group $(\mathrm{n}=44)$ and the Neoral group ( $\mathrm{n}=61$ ): creatinine clearance: $56.0 \mathrm{ml} / \mathrm{min}$ versus $50.0 \mathrm{ml} / \mathrm{min}$ $(P=0.12), \log$ transformed proteinuria: $1.28 \mathrm{~g} / \mathrm{mol}$ creatinine versus $1.20 \mathrm{~g} / \mathrm{mol}$ creatinine $(P=0.39)$, fractional uric acid clearance: $9.5 \%$ versus $9.7 \%(P=0.87)$, and mean blood pressure: $97.7 \mathrm{mmHg}$ versus $95.0 \mathrm{mmHg}(P=0.25)$. In the tacrolimus group still less patients used anti-hypertensive drugs (mean 1.3 versus 2.3, $P<0.001)$. The differences in cholesterol $(5.4 \mathrm{mmol} / 1$ versus $5.8 \mathrm{mmol} / 1, P=0.10)$ and the percentage of patients on lipid-lowering drugs $(27.3 \%$ versus $38.7 \%$, $P=0.37$ ) were attenuated, but still less favourable in the Neoral group. We also analyzed the possible influence of MMF on the secondary outcome. All clinical outcome parameters were well comparable between the patients treated with tacrolimus with MMF and the patients treated with tacrolimus without MMF. Thus, MMF seems to play no role on these parameters.

In previous studies comparing tacrolimus with Sandimmune, the incidence of post-transplant diabetes was higher in patients taking tacrolimus and ranged from $15 \%$ to $20 \% 19-21,23,36$. We also found a comparable incidence of $18.5 \%$ in the tacrolimus group, with a similar need for treatment with insulin, as in the Neoral group at any time after transplantation. After tapering the dose of tacrolimus and withdrawing steroids, $70 \%$ of the patients with post-transplant diabetes could stop 
taking all medication. The reversibility of post-transplant diabetes is in accordance with other studies ${ }^{19,20,36,37}$. The percentage of patients who persisted on antidiabetic medication was similar with tacrolimus and Neoral.

In the first interim report of the ongoing prospective study, Morris-Stiff et al. reported a similar incidence of diabetes with tacrolimus and $\mathrm{Neoral}^{27}$, but in that study tacrolimus was initially relatively low-dosed.

In summary, this study compared tacrolimus-based and Neoral-based immunosuppression. A substantial lower rate of first and relapsing acute rejections and fewer failures due to immunological causes were observed with tacrolimus. Furthermore, a higher proportion of patients could safely have steroids withdrawn and be put on monotherapy. We hereby extend the findings that tacrolimus is more effective than either Sandimmune or Neoral. Renal function was comparable, but the cardiovascular risk profile was more favourable, with lower total cholesterol and fewer patients needing drugs for the treatment of hypertension and hypercholesterolemia. In the initial phase after transplantation, the incidence of post-transplant diabetes mellitus was higher, but after lowering the dose of tacrolimus and withdrawing from steroids, the need to treat posttransplant diabetes was identical. 


\section{References}

1. Calne RY, Rolles K, White DJG, Thiru S, Evans DB, McMaster P, Dunn DC, Craddock GN, Henderson RG, Aziz S and Lewis P. Cyclosporin initially as the only immunosuppressant in 34 recipients of cadaveric organs: 32 kidneys, 2 pancreases, and 2 livers. Lancet 1979;2:1033-6.

2. European Multicentre Trial Group. Cyclosporine in cadaveric renal transplantation: one-year follow-up of a multicentre trial. Lancet 1983;2:986-9.

3. Hall BM, Tiller DJ, Hardie I, Mahony J, Mathew T, Thatcher G, Miach P, Thomson N and Sheil AG. Comparison of three immunosuppressive regimens in cadaver renal transplantation: long-term cyclosporine, short-term cyclosporine followed by azathioprine and prednisolone, and azathioprine and prednisolone without cyclosporine. N Engl J Med 1988;318:1499-507.

4. The Canadian Multicentre Transplant Study Group. A randomized clinical trial of cyclosporine in cadaveric renal transplantation. N Engl J Med 1983;309:809-15.

5. Gjertson DW, Cecka JM and Terasaki PI. The relative effects of FK506 and cyclosporine on short- and long-term kidney graft survival. Transplantation 1995;60:1384-8.

6. Basadonna GP, Matas AJ, Gillingham KJ, Payne WD, Dunn DL, Sutherland DER, Gores PF, Gruessner RWG and Najarian JS. Early versus late acute renal allograft rejection: impact on chronic rejection. Transplantation 1993;55:993-5.

7. Basadonna GP, Matas AJ, Gillingham KJ, Payne WD, Dunn DL, Sutherland DER, Gores PF, Gruessner RWG, Arrazola L and Najarian JS. Relationship between early vs late acute rejection and onset of chronic rejection in kidney transplantation. Transplant Proc 1993;25: 910-1.

8. Ishikawa A, Flechner SM, Goldfarb DA, Myles JL, Modlin CS, Boparai N, Papajcik D, Mastroianni B and Novick AC. Quantitative assessment of the first acute rejection as a predictor of renal transplant outcome. Transplantation 1999;68:1318-24.

9. Land W, Schneeberger H, Schleibner S, Illner W-D, Abendroth D, Hillebrand G, Gokel JM, Albert E and Fornara P. Long-term results in cadaveric renal transplanttation under cyclosporin therapy. Transplant Proc 1991;23:1244-6.

10. Lindholm A, Ohlman S, Albrechtsen D, Tufveson G, Persson H and Persson NH. The impact of acute rejection episodes on long-term graft function and outcome in 1347 primary renal transplants treated by 3 cyclosporine regimens. Transplantation 1993;56:307-15.

11. Marcén R, Pascual J, Orofino L, Cal MA de la, Teruel JL, Villafruela JJ, Rivera ME, Burgos FJ, Mampaso F and Ortuño J. The effect of delayed graft function and early graft rejection on renal transplant outcome. Transplant Proc 1998;30:1776-7.

12. Matas A. Chronic rejection in renal transplant recipients - risk factors and correlates. Clin Transplant 1994;8:332-5.

13. Solez K, Vincenti F and Filo RS. Histopathologic findings from 2-year protocol biopsies from a U.S. multicenter kidney transplant trial comparing tacrolimus versus cyclosporine. A report of the FK506 Kidney Transplant Study Group. Transplantation 1998;66:1736-40.

14. Vanrenterghem YFC. Acute rejection and renal allograft outcome. Nephrol Dial Transplant 1995;10(S1):29-31.

15. Friman $\mathrm{S}$ and Bäckman L. A new microemulsion formulation of cyclosporin. Pharmacokinetic and clinical features. Clin Pharmacokinet 1996;30:181-93. 
16. Keown P and Niese D on behalf of the International Sandimmun Neoral Study Group. Cyclosporine microemulsion increases drug exposure and reduces acute rejection without incremental toxicity in de novo renal transplantation. Kidney Int 1998;54:938-44.

17. Niese D on behalf of the International Sandimmun Neoral Study Group. A double-blind randomized study of Sandimmun Neoral versus Sandimmun in new renal transplant recipients: results after twelve months. Transplant Proc 1995;27:1849-56.

18. Pollard SG, Lear PA, Ready AR, Moore RH and Johnson RWG on behalf of the UK Neoral Renal Study Group. Comparison of microemulsion and conventional formulations of cyclosporin A in preventing acute rejection in de novo kidney transplant patients. Transplantation 1999;68: 1325-31.

19. Jensik SC and the FK506 Kidney Transplant Study Group. Tacrolimus (FK 506) in kidney transplantation: Three-year survival results of the US multicenter, randomized, comparative trial. Transplant Proc 1998;30:1216-8.

20. Mayer AD, Dmitrewski J, Squifflet J-P, Besse T, Grabensee B, Klein B, Eigler FW, Heemann U, Pichlmayr R, Behrend M, Vanrenterghem Y, Donck J, Hooff J van, Christiaans M, Morales JM, Andres A, Johnson RWG, Short C, Buchholz B, Rehmert N, Land W, Schleibner S, Forsythe JLR, Talbot D, Neumayer H-H, Hauser I, Ericzon B-G, Brattström C, Claesson K, Mühlbacher F and Pohanka E. Multicenter randomized trial comparing tacrolimus (FK 506) and cyclosporin in the prevention of renal allograft rejection. A report of the European Tacrolimus Multicenter Renal Study Group. Transplantation 1997;64:436-43.

21. Pirsch JD, Miller J, Deierhoi MH, Vincenti F and Filo RS for the FK506 Kidney Transplant Study Group. A comparison of tacrolimus (FK506) and cyclosporine for immunosuppression after cadaveric renal transplantation. Transplantation 1997;63:977-83.

22. Schleibner S, Krauss M, Wagner K, Erhard J, Christiaans M, Hooff J van, Buist L and Mayer D. FK 506 versus cyclosporin in the prevention of renal allograft rejection - European pilot study: six week results. Transpl Int 1995;8:86-90.

23. Vincenti F, Laskow DA, Neylan JF, Mendez R and Matas AJ. One-year follow up of an openlabel trial of FK506 for primary kidney transplantation. Transplantation 1996;61:1576-81.

24. Knoll GA and Bell RC. Tacrolimus versus cyclosporin for immunosuppression in renal transplantation: Meta-analysis of randomised trials. Br Med J 1999;318:1104-7.

25. Ghasemian SR, Light JA, Currier C, Sasaki TM and Aquino A. Tacrolimus vs Neoral in renal and renal/pancreas transplantation. Clin Transplant 1999;13:123-5.

26. Jurewicz WA. Immunological and non-immunological risk factors with tacrolimus and Neoral in renal transplant recipients: An interim report. Transplant Proc 1999;31(S7A):64S-6.

27. Morris-Stiff G, Ostrowski K, Balaji V, Moore R, Darby C, Lord R and Jurewicz WA. Prospective randomised study comparing tacrolimus (Prograf) and cyclosporine (Neoral) as primary immunosuppression in cadaveric renal transplants at a single institution: interim report of the first 80 cases. Transpl Int 1998;11(S1):S334-6.

28. Claesson K, Mayer AD, Squifflet J-P, Grabensee B, Eigler FW, Behrend M, Vanrenterghem Y, Hooff J van, Morales JM, Johnson RWG, Buchholz B, Land W, Forsythe JLR, Neumayer H$H$, Ericzon B-G and Mühlbacher F. Lipoprotein patterns in renal transplant patients: A comparison between FK 506 and cyclosporin A patients. Transplant Proc 1998;30:1292-4.

29. Hohage H, Arlt M, Brückner D, Dietl KH, Zidek W and Spieker S. Effects of cyclosporin A and FK 506 on lipid metabolism and fibrinogen in kidney transplant recipients. Clin Transplant 1997;11:225-30. 
30. Hohage H, Brückner D, Arlt M, Buchholz B, Zidek W and Spieker C. Influence of cyclosporin $\mathrm{A}$ and FK506 on $24 \mathrm{~h}$ blood pressure monitoring in kidney transplant recipients. Clin Nephrol 1996;45:342-4.

31. Radermacher J, Meiners M, Bramlage C, Kliem V, Behrend M, Schlitt HJ, Pichlmayr R, Koch $\mathrm{KM}$ and Brunkhorst R. Pronounced renal vasoconstriction and systemic hypertension in renal transplant recipients treated with cyclosporin A versus FK 506. Transpl Int 1998;11:3-10.

32. Shapiro R, Jordan M, Scantlebury V, Fung J, Jensen C, Tzakis A, McCauley J, Carroll P, Ricordi C, Demetris AJ, Mitchell S, Jain A, Iwaki Y, Kobayashi M, Reyes J, Todo S, Hakala TR, Simmons RL and Starzl TE. FK 506 in clinical kidney transplantation. Transplant Proc 1991;23:3065-7.

33. Racusen LC, Solez K, Colvin RB, Bonsib SM, Castro MC, Cavallo T, Croker BP, Demetris AJ, Drachenberg CB, Fogo AB, Furness P, Gaber LW, Gibson IW, Glotz D, Goldberg JC, Grande JG, Halloran PF, Hansen HE, Hartley B, Hayry PJ, Hill CM, Hoffman EO, Hunsicker LG, Lindblad AS, Marcussen N, Mihatsch MJ, Nadasdy T, Nickerson P, Olsen TS, Papadimitriou JC, Randhawa PS, Rayner DC, Roberts I, Rose S, Rush D, Salinas-Madrigal L, Salomon DR, Sund S, Taskinen E, Trpkov K and Yamaguchi Y. The Banff 97 working classification of renal allograft pathology. Kidney Int 1999;55:713-23.

34. Cockcroft DW and Gault MH. Prediction of creatinine clearance from serum creatinine. Nephron 1976;16:31-41.

35. Ruggenenti P, Gaspari F, Perna A and Remuzzi G. Cross sectional longitudinal study of spot morning urine protein:creatinine ratio, 24 hour urine protein excretion rate, glomerular filtration rate, and end stage renal failure in chronic renal disease in patients without diabetes. Br Med J 1998;316:504-9.

36. Shapiro R, Jordan ML, Scantlebury VP, Vivas C, Fung JJ, McCauley J, Randhawa P, Demetris AJ, Irish W, Jain A, Mitchell S, Hakala TR, Simmons RL and Starzl TE. A prospective, randomized trial of FK 506/prednisone vs FK 506/azathioprine/prednisone in renal transplant patients. Transplant Proc 1995;27:814-7.

37. Yokoyama I, Uchida K, Fukao K, Ochiai T, Takahashi K, Endo T, Oshima S, Ishibashi M, Takahara S, Iwasaki Y, Ota K, Takagi H and Sonoda T for the Japanese FK 506 Study Group. FK 506: Long-term study in kidney transplantation. Transplant Proc 1995;27:818-21.

38. Vanrenterghem Y, Squifflet JP, Forsythe J, Heeman U, Backman L, Taube D, Morales JM, Ekberg H, Hooff J van, Zanker B, Dietl KH, Talbot D, Hauser I, Tyden G, Claesson K and Mühlbacher F. Co-administration of tacrolimus and mycophenolate mofetil in cadaveric renal transplant recipients. Transplant Proc 1998;30: 1290-1.

39. Squifflet JP, Hooff JP van and Vanrenterghem Y. The Benelux experience with the combination of tacrolimus and mycophenolate mofetil. Transplant Proc 1999;31(S7A):72S-4.

40. Shapiro R, Jordan ML, Scantlebury VP, Vivas C, Marsh JW, McCauley J, Johnston J, Randhawa P, Irish W, Gritsch HA, Naraghi R, Hakala TR, Fung JJ and Starzl TE. A prospective, randomized trial of tacrolimus/prednisone versus tacrolimus/prednisone/ mycophenolate mofetil in renal transplant recipients. Transplantation 1999;67:411-5.

41. Isaacs RB, Nock SL, Spencer CE, Connors AF jr, Wang X-Q, Sawyer R and Lobo PI. Racial disparities in renal transplant outcomes. Am J Kidney Dis 1999;34:706-12.

42. Neylan JF for the US Renal Transplant Mycophenolate Mofetil Study Group. Immunosuppressive therapy in high-risk transplant patients. Dose-dependent efficacy of mycophenolate mofetil in African-American renal allograft recipients. Transplantation 1997; 64:1277-82. 
43. Roodnat JI, Zietse R, Mulder PGH, Rischen-Vos J, Gelder T van, IJzermans JNM and Weimar W. The vanishing importance of age in renal transplantation. Transplantation 1999;67:576-80.

44. Senel MF, Buren CT van, Welsh M and Kahan BD. Impact of early cyclosporin average blood concentration on early kidney transplant failure. Transpl Int 1998;11:46-52.

45. Mihatsch MJ, Kyo M, Morozumi K, Yamaguchi Y, Nickeleit V and Ryffel B. The side effects of ciclosporin-A and tacrolimus. Clin Nephrol 1998;49:356-63.

46. Undre NA, Hooff J van, Christiaans M, Vanrenterghem Y, Donck J, Heeman U, Kohnle M, Zanker W, Morales JM, Andrés A, Schäfer A and Stevenson P. Low systemic exposure to tacrolimus correlates with acute rejection. Transplant Proc 1999;31:296-8.

47. Richards E, Schleibner S, Talbot D and the European Tacrolimus Multicentre Renal Study Group. An exploratory analysis of prognostic factors for patient outcome during the first year following renal transplantation. Transplant Proc 1998;30:1386-8.

48. Jordan ML, Naraghi R, Shapiro R, Smith D, Vivas CA, Scantlebury VP, Gritsch HA, McCauley J, Randhawa P, Demetris AJ, McMichael J, Fung JJ and Starzl TE. Tacrolimus rescue therapy for renal allograft rejection - five-year experience. Transplantation 1997;63:223-8.

49. Morrissey PE, Gohh R, Shaffer D, Crosson A, Madras PN, Sahyoun AI and Monaco AP. Correlation of clinical outcomes after tacrolimus conversion for resistant kidney rejection or cyclosporin toxicity with pathologic staging by the Banff criteria. Transplantation 1997;63: 845-8.

50. Woodle ES, Cronin D, Newell KA, Millis JM, Bruce DS, Piper JB, Haas M, Josephson MA and Thistlethwaite JR. Tacrolimus therapy for refractory acute renal allograft rejection. Definition of the histologic response by protocol biopsies. Transplantation 1996;62: 906-10.

51. Woodle ES, Thistlethwaite JR, Gordon JH, Laskow D, Deierhoi MH, Burdick J, Pirsch JD, Sollinger H, Vincenti F, Burrows L, Schwartz B, Danovitch GM, Wilkinson AH, Shaffer D, Simpson MA, Freeman RB, Rohrer RJ, Mendez R, Aswad S, Munn SR, Wiesner RH, Delmonico FL, Neylan J and Whelchel J. A multicenter trial of FK506 (tacrolimus) therapy in refractory acute renal allograft rejection. A report of the Tacrolimus Kidney Transplantation Rescue Study Group. Transplantation 1996;62:594-9.

52. Bertoni E, Zanazzi M, Maria L di, Moscarelli L, Piperno R, Conti P, Dedola G, Bandini S, Tosi $\mathrm{P}$ and Salvadori M. Long-term steroid side effects in renal transplantation need a safe steroid withdrawal: a single-center experience. Transplant Proc 1998;30:1303-4.

53. Ahsan N, Hricik D, Matas A, Rose S, Tomlanovich S, Wilkinson A, Ewell M, McIntosh M, Stablein D and Hodge E for the Steroid Withdrawal Group Prednisone withdrawal in kidney transplant recipients on cyclosporin and mycophenolate mofetil - a prospective randomized study. Transplantation 1999;68:1865-74.

54. Dimény E, Wahlberg J, Lithell $\mathrm{H}$ and Fellström B. Hyperlipidaemia in renal transplantation risk factor for long-term graft outcome. Eur J Clin Invest 1995;25:574-83.

55. Peschke B, Scheuermann, Geiger H, Bölscher S, Kachel H-G and Lenz T. Hypertension is associated with hyperlipidemia, coronary heart disease and chronic graft failure in kidney transplant recipients. Clin Nephrol 1999;51:290-5. 


\section{Chapter 3}

\section{Suppression of the hypothalamic- pituitary-adrenal axis in renal transplantation with steroid-included triple therapy}

JMM Boots, ECH van den Ham, MHL Christiaans, JP van Hooff 


\section{Abstract}

\section{Introduction}

There is more tendency nowadays to withdraw steroids three to six months posttransplantation. The risk of adrenal insufficiency and the degree of suppression of the hypothalamic-pituitary-adrenal axis (HPA-axis), however, have never been clearly defined. Secondly, it was tried to identify a tool to detect patients at risk of symptoms after rapid steroid withdrawal.

\section{Methods}

In this prospective cohort study, the functionality of the HPA-axis was determined three $(n=42)$ and six $(n=25)$ months post-transplantation with the $0.25 \mathrm{mg}$ short synacthen test (SST). Steroids were tapered after transplantation to $10 \mathrm{mg}$ prednisolone from week 5 onwards. Immunosuppression consisted further of tacrolimus and mycophenolate mofetil. Rapid steroid withdrawal in two weeks was performed in 14 patients after three months and in ten patients after six months post-transplantation. Normal lower level for ACTH was $20 \mathrm{ng} / \mathrm{l}$ and for cortisol $200 \mathrm{nmol} / \mathrm{l}$. The response on the SST was considered appropriate if the stimulated cortisol after 30 minutes was $\geq 550 \mathrm{nmol} / 1$. Three and six months data were compared by the paired samples statistics.

\section{Results}

Baseline values at month 3: ACTH was $<20 \mathrm{ng} / 1 \mathrm{in} 51 \%$ of the patients; cortisol was $<200 \mathrm{nmol} / 1$ in $31 \%$ and $<100 \mathrm{nmol} / 1$ in $7 \%$; at month 6 , ACTH was $<20 \mathrm{ng} / \mathrm{l}$ in 46\%; cortisol was $<200 \mathrm{nmol} / 1$ in $36 \%$ and $<100 \mathrm{nmol} / 1$ in $12 \%$. Both mean baseline cortisol: 325 versus $272 \mathrm{nmol} / \mathrm{l}(P=0.05)$ and stimulated cortisol: 542 versus $491 \mathrm{nmol} / 1(P=0.05)$ were lower after six months, whereas mean ACTH was comparable. The SST was inappropriate in 55\% at month 3 and in $52 \%$ at month 6 . Only after six months, rapid steroid withdrawal resulted in mild symptoms which might be attributed to adrenal insufficiency in three of the ten patients: orthostasis $(n=3)$ and weakness $(n=1)$. None of the patients came in a stressful situation. A baseline cortisol above $400 \mathrm{nmol} / \mathrm{l}$ or a stimulated cortisol above $550 \mathrm{nmol} / 1$ indicates patients, in which rapid steroid withdrawal is reasonably safe.

\section{Conclusions}

Suppression of the HPA-axis is common with $10 \mathrm{mg}$ prednisolone maintenance therapy and increases with the duration of therapy. Rapid steroid withdrawal seems to be safe after three months. Afterwards, a two-step cortisol analysis can rule out patients at risk of developing clinical symptoms. 


\section{Introduction}

Steroids have made a major contribution towards the success of renal transplantation and therefore they have been part of almost all induction and maintenance immunosuppressive regimens. Because of their serious long-term side effects and the availability of more effective immunosuppressants, there is more and more tendency nowadays to withdraw steroids after the first three to six months post-transplantation. In the case of severe side effects like infections, new-onset or poorly controlled diabetes mellitus, it may even be desirable to withdraw steroids more rapidly. However, steroids suppress the hypothalamicpituitary-adrenal axis (HPA-axis) and rapid steroid withdrawal may lead to adrenal insufficiency and life-threatening conditions. Since the early publications, stress doses of steroids are recommended for stressful episodes like surgery. Suppression of the HPA-axis already results from one to four weeks' highly dosed steroid schedules ${ }^{1}$. Based on cross-sectional data, it has been concluded that the suppressive effect of a certain steroid depends on the height of the dose, the duration of the therapy, and the time of administration during the day ${ }^{2}$.

So far, it is unknown which strategy of steroid withdrawal is still a safe procedure with regard to the risk of adrenal insufficiency. After searching the Medline database and specific literature, prospective studies evaluating the risk of adrenal insufficiency with the common dose of steroids used nowadays from transplantation onwards (10 $\mathrm{mg}$ predniso(lo)ne ) have not been published.

Thus, the magnitude of suppression of the HPA-axis with the common steroid doses and the risk of rapid steroid withdrawal three to six months posttransplantation remain unclear. In this prospective study, we evaluated the functionality of the HPA-axis in a cohort of renal transplant recipients scheduled for rapid discontinuation of their maintenance therapy of $10 \mathrm{mg}$ prednisolone three or six months post-transplantation, at first to evaluate how many patients have suppression of the HPA-axis, and secondly to identify a tool to detect patients who may especially be at risk.

\section{Patients and methods}

\section{Patients}

Between September 1998 and November 1999, 53 patients participated in a multicentre trial, consisting of triple therapy with tacrolimus, mycophenolate-mofetil (MMF) and steroids. In this trial, patients were randomized at the time of transplantation to continue triple therapy or to have either MMF or steroids withdrawn three months after transplantation (Figure 3.1). The patients were then 
followed for another three months. All patients gave separate informed consent for the present study.

\section{Immunosuppressive protocol}

Steroids were started with an intravenous bolus of $125 \mathrm{mg}$ methylprednisolone at the initiation of surgery. This dose was repeated at day 1 post-transplantation. From day 2 until day 15, patients received $20 \mathrm{mg}$ prednisolone orally once daily. After week 2, the dose was reduced to $15 \mathrm{mg}$ and from week 5 onwards, the patients used $10 \mathrm{mg}$. The prednisolone was taken at 8.00 A.M.

Tacrolimus was administered orally $0.1 \mathrm{mg} / \mathrm{kg}$ b.i.d. and started within 12 hours prior to surgery. After transplantation the dose was adjusted to 12-hours trough levels, measured by a whole blood micro-particle immunoassay (IMx, Abbot Laboratories, Abbott Park, IL, USA). The target levels were around $10 \mathrm{ng} / \mathrm{ml}$ after three months and 5-7 $\mathrm{ng} / \mathrm{ml}$ after six months.

MMF was administered orally $500 \mathrm{mg}$ b.i.d., starting at day one or two posttransplantation.

Acute rejections were treated with a course of three doses of $500 \mathrm{mg}$ intravenous methylprednisolone on alternate days. Steroid resistant or vascular rejections were treated with a ten-day course of rabbit-anti-thymocyte globulin (r-ATG). Prednisolone reduction in these patients was restricted to $5 \mathrm{mg}$, unless the rejection was mild (small rise in serum creatinine or 1997 Banff-classification $^{3}$ as borderline or $1 \mathrm{~A})$.

In the patients, who were randomized for steroid withdrawal, prednisolone was withdrawn in two weeks after month three. In the first week, the dose of prednisolone was reduced to $5 \mathrm{mg}$ and in the second week to $2.5 \mathrm{mg}$. In ten patients, the steroids were withdrawn in a similar way after six months. After discontinuation of the steroids, patients were followed on the outpatient clinic for symptoms of adrenal insufficiency: weakness, fatigue, weight loss, anorexia, gastro-intestinal disturbances, salt craving, dizziness, and orthostasis.

The cumulative prednisolone equivalence dose was calculated by considering the prednisolone equivalent strength of methylprednisolone as 1.25 and of hydrocortisone as 0.25 . 


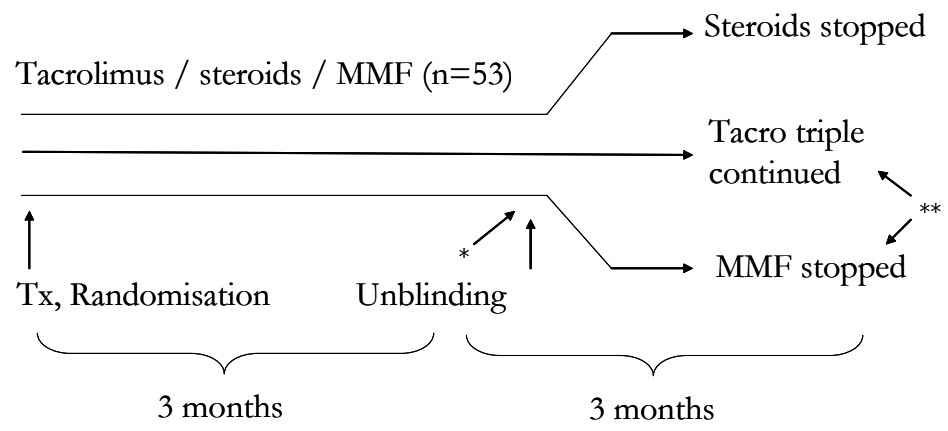

Figure 3.1 Study design. The patients were randomized at the time of transplantation. Just before unblinding the randomisation at month 3 , the first synacthen test was performed. In the patients who were randomized to continue steroids, the second synacthen test was performed at month 6 .

* Synacthen $1(\mathrm{n}=42) ; * *$ Synacthen $2(\mathrm{n}=25)$

\section{Suppression of the HPA-axis}

The short Synacthen-test (SST) was used to evaluate the HPA-axis. The tests were performed in the morning between 8.30 and 9.00 at month 3 post-transplantation prior to the unblinding of the randomisation and just before month 6 in the patients who had their steroids continued (Figure 3.1). Blood samples were taken after cannulation of an antecubital vein with an intravenous catheter just prior to and 30 minutes after intravenous administration of $0.25 \mathrm{mg}$ Synacthen (Novartis, Basel, Switzerland). The patients were in the sitting position in a comfortable chair. Adreno-corticotrophic hormone (ACTH) was measured with an immunoradiometric assay (Nichols Institute Diagnostics, Zwanenburg, The Netherlands). The reference values in our laboratory for 9.00 AM are 20-60 ng/l. The lowest level of detection is $5 \mathrm{ng} / \mathrm{l}$. Plasma cortisol was measured with a lumino-enzymeimmunoassay (Immulite ${ }^{\circledR}$ 2000, Diagnostics Product Corporation, Breda, The Netherlands). The reference values for $9.00 \mathrm{AM}$ in our laboratory are 200-700 nmol/1. A cortisol level below $100 \mathrm{nmol} / 1$ is consistent with obvious adrenal suppression ${ }^{4,5}$. An appropriate function of the HPA-axis was defined as an increase in plasma cortisol in the SST above $550 \mathrm{nmol} / 1$ as advized by Oelkers ${ }^{6}$ and May et al. ${ }^{7}$.

\section{Statistical analysis}

Statistical analyses were performed with the SPSS software package for Windows, version 10.0 (SPSS, Inc, Chicago, IL.). The data of the patients who were eligible 
at the time points of the first and second test were compared with either the independent samples t-test for continuous data, the Mann-Whitney test in the case of a non-parametrical distribution or a small subgroup, or the $\chi^{2}$-test for categorical variables. Comparisons between month 3 and month 6 were performed with the paired samples t-test for continuous data or the McNemar test for categorical variables. Pearson's correlations were used to evaluate possible correlations for continuous data and Spearman's rho correlations were used otherwise.

A multivariate analysis was performed with potential factors influencing the test results as depending variables: age (year), body mass index $\left(\mathrm{kg} / \mathrm{m}^{2}\right)$, serum creatinine at month 3 or month $6(\mu \mathrm{mol} / \mathrm{l})$, gender (male versus female), and acute rejection (yes versus no). A linear regression model was used to evaluate the effect of these parameters on the baseline cortisol level at both month 3 and month 6 . A logistic regression model was used to evaluate the effect of these parameters on the response of the SST. Both a forward selection and a backward elimination technique of the factors were applied.

Data are expressed as mean $\pm \mathrm{SD}$ unless otherwise specified. $P$-values less than 0.05 were considered significant.

\section{Results}

\section{Patients}

Fifty-three patients participated in this cohort study. Their mean age was $55.1 \pm 12.1$ years and their mean body mass index was $25.9 \pm 4.5 \mathrm{~kg} / \mathrm{m}^{2}$. Thirtyeight patients were male $(72 \%)$. Fifty-one patients $(96 \%)$ belonged to the Caucasian race and two patients $(4 \%)$ were black.

Forty-two patients were tested at month 3 and 25 patients were tested at month 6 . Eleven patients were not eligible for the first SST at month 3. In seven patients, the graft had failed. The indications of the other four patients were as follows: withdrawal of informed consent $(n=1)$, lost to follow-up $(n=1)$, and premature prednisolone reduction because of severe infection $(n=2)$. After unblinding the randomisation at month 3,15 patients had been randomized to prednisolone withdrawal in two weeks, 14 to continuation of triple therapy and 13 to stop MMF. In both latter groups, one patient was not eligible for the second test: graft failure due to ongoing rejection (triple group), and prednisolone withdrawal because of infection (MMF stop group). Both after three and six months, there were no statistical differences in the baseline demographics between the tested patients and the patients that could not be tested. The results after six months between the triple group and the MMF stop group were comparable (cortisol: $P>0.2$, t-test and ACTH: $P>0.1$, Mann-Whitney test). 
The mean cumulative prednisolone equivalence dose was $1526 \pm 480 \mathrm{mg}$ after three months and $2310 \pm 20 \mathrm{mg}$ after six months in the tested patients.

\section{Synacthen results at month 3}

The first test was performed at a median of 77 days post-transplantation (range: 59-87 days).

ACTH was not determined in one patient.

The mean baseline ACTH level was $22 \pm 13 \mathrm{ng} / \mathrm{l}$. ACTH was below $20 \mathrm{ng} / \mathrm{l}$ in 21 patients (51\%) and below the level of detection (5 ng/l) in one patient (2\%).

The mean baseline cortisol was $317 \pm 175 \mathrm{nmol} / \mathrm{l}$. Cortisol was below $200 \mathrm{nmol} / 1$ in 13 patients (31\%) and below $100 \mathrm{nmol} / \mathrm{l}$ in three patients $(7 \%)$.

The response on the synacthen test was insufficient (stimulated cortisol $<550 \mathrm{nmol} / \mathrm{l})$ in 23 patients (55\%).

\section{Synacthen results at month 6}

The second test was performed at a median of 171 days post-transplantation (range: 161-198 days). Also after six months, ACTH was not determined in one patient.

The mean baseline ACTH level was $24 \pm 16 \mathrm{ng} / \mathrm{l}$. ACTH was below $20 \mathrm{ng} / \mathrm{l}$ in 11 patients (46\%) and below $5 \mathrm{ng} / \mathrm{l}$ in two patients $(8 \%)$.

The mean baseline cortisol was $272 \pm 153 \mathrm{nmol} / \mathrm{l}$. Cortisol was below $200 \mathrm{nmol} / \mathrm{l}$ in nine patients $(36 \%)$ and below $100 \mathrm{nmol} / \mathrm{l}$ in three patients $(12 \%)$.

The response on the synacthen test was insufficient (stimulated cortisol $<550 \mathrm{nmol} / \mathrm{l}$ ) in 13 patients (52\%).

\section{Comparison between month 3 and month 6}

Twenty-five patients were tested both after three and after six months. Their results are presented in Table 3.1. In these patients, both the baseline and the stimulated cortisol were lower after six months, whereas ACTH was similar. Both the number of patients with suppression of ACTH below $20 \mathrm{ng} / \mathrm{l}$ and the number of patients with suppression of the baseline cortisol below $200 \mathrm{nmol} / \mathrm{l}$ were comparable. Although the number of patients with a suppressed ACTH level below $5 \mathrm{ng} / \mathrm{l}$ or a suppressed cortisol level below $100 \mathrm{nmol} / \mathrm{l}$ were higher after six months, this difference did not reach statistical significance. The number of patients with an inappropriate response on synacthen at three and six months post-transplantation was identical. 
Table 3.1 The data of the patients who were tested twice

Twenty-five patients were tested both after three and after six months. Both baseline and stimulated cortisol were significantly lower after six months $(P=0.05$, paired samples t-test).

\begin{tabular}{lccc}
\hline & Month 3 & Month 6 & $\boldsymbol{P}$-value \\
\hline ACTH $(\mathrm{ng} / \mathrm{l})$ & $23 \pm 11$ & $24 \pm 16$ & $0.58^{1}$ \\
ACTH $<20 \mathrm{ng} / \mathrm{l}$ & $12(50 \%)$ & $11(46 \%)$ & $0.63^{2}$ \\
ACTH $<5 \mathrm{ng} / \mathrm{l}$ & $0(0 \%)$ & $2(8 \%)$ & $0.50^{2}$ \\
Baseline cortisol $(\mathrm{nmol} / \mathrm{l})$ & $325 \pm 153$ & $272 \pm 153$ & $0.05^{1}$ \\
Stimulated cortisol $(\mathrm{nmol})$ & $542 \pm 175$ & $491 \pm 196$ & $0.05^{1}$ \\
Baseline cortisol $<200 \mathrm{nmol} / 1$ & $7(28 \%)$ & $9(36 \%)$ & $0.73^{2}$ \\
Baseline cortisol $<100 \mathrm{nmol} / \mathrm{l}$ & $1(4 \%)$ & $3(12 \%)$ & $0.63^{2}$ \\
Inappropriate response SST & $13(52 \%)$ & $13(52 \%)$ & $1.00^{2}$ \\
\hline
\end{tabular}

${ }^{1}$ Paired samples t-test; ${ }^{2} \mathrm{McNemar}$ test

\section{Rejectors}

Seven patients $(13 \%)$ experienced one or more episodes of acute rejection for which they were treated with methylprednisolone. Five of them participated in the synacthen test at month 3 and two in the test at month 6 . At three months the baseline ACTH: $14.5 \pm 9.3 \mathrm{ng} / \mathrm{l}$ versus $22.5 \pm 12.8 \mathrm{ng} / \mathrm{l}(P=0.21$, Mann-Whitney test) and the baseline cortisol: $219 \pm 152 \mathrm{nmol} / 1$ versus $330 \pm 176 \mathrm{nmol} / 1(P=0.19$, Mann-Whitney test) were lower in the rejectors versus the non-rejectors, but the differences were not statistically significant. At month 3, $80 \%$ of the rejectors had an inappropriate response on the synacthen test versus $51 \%$ of the non-rejectors $\left(P=0.23, \chi^{2}\right.$-test). At month 6 , the results between the rejectors and the nonrejectors were similar.

\section{Correlations}

The correlation coefficients and the statistical significance are presented in Table 3.2. The ACTH level at month 3 was weakly correlated with the level at month 6 . At month 3, the ACTH level did not correlate with the baseline cortisol level. This was in contrast to month six, when there was a fair correlation. There was no correlation between an ACTH level below $20 \mathrm{ng} / \mathrm{l}$ and the response on the synacthen test both after three and after six months. All patients with an ACTH level $<5 \mathrm{ng} / \mathrm{l}$ had an inappropriate response on the SST, both after three and after six months. 
Table 3.2 Correlations. SST implies the short synacthen test. Pearson's correlations are used except for the correlations of the response on the SST (Spearman's rho correlations).

\begin{tabular}{llcc}
\hline Variable 1 & Variable 2 & Correlation Coefficient & $\boldsymbol{P}$-value \\
\hline ACTH month 3 & ACTH month 6 & 0.48 & 0.02 \\
ACTH month 3 & baseline cortisol month 3 & 0.30 & 0.06 \\
ACTH month 6 & baseline cortisol month 6 & 0.66 & $<0.001$ \\
Baseline cortisol month 3 & baseline cortisol month 6 & 0.67 & $<0.001$ \\
Stimulated cortisol month 3 & stimulated cortisol month 6 & 0.78 & $<0.001$ \\
Baseline cortisol month 3 & stimulated cortisol month 3 & 0.90 & $<0.001$ \\
Baseline cortisol month 6 & stimulated cortisol month 6 & 0.85 & $<0.001$ \\
Cortisol <200 nmol/1 month 3 & inappropriate SST month 3 & 0.61 & $<0.001$ \\
Cortisol <200 nmol/1 month 6 & inappropriate SST month 6 & 0.55 & 0.004 \\
\hline
\end{tabular}

Both baseline and stimulated cortisol at month 3 were fairly correlated with the results at month 6 . The baseline cortisol levels correlated well with the stimulated values. All patients with a baseline cortisol below $100 \mathrm{nmol} / 1 \mathrm{had}$ an inappropriate response on the synacthen test (Figure 3.2). A suppressed baseline cortisol below $200 \mathrm{nmol} / \mathrm{l}$ correlated fairly with an inappropriate response on the synacthen test. The variation in cumulative prednisolone equivalence dose resulted from the administration of methylprednisolone in the rejectors. Since the variation in the other patients was marginal, a reliable estimation of the correlations between the cumulative prednisolone equivalence dose and the results of the tests could not be performed.

\section{Multivariate analysis of risk factors}

At month 6 , but not at month 3 , both body mass index $(\beta=34.5 \mathrm{nmol} / 1$ per $\mathrm{kg} / \mathrm{m}^{2} ; 95 \%$ confidence interval: $\left.6.7-62.2 \mathrm{nmol} / 1, P=0.02\right)$ and age $(\beta=-6.8 \mathrm{nmol} / 1$ per year; $95 \%$ confidence interval: $-1.6--12.1 \mathrm{nmol} / \mathrm{l}, P=0.01$ ) were related to the baseline cortisol level. None of the included factors was significantly related to an inappropriate response on the synacthen test, both after three and after six months. 

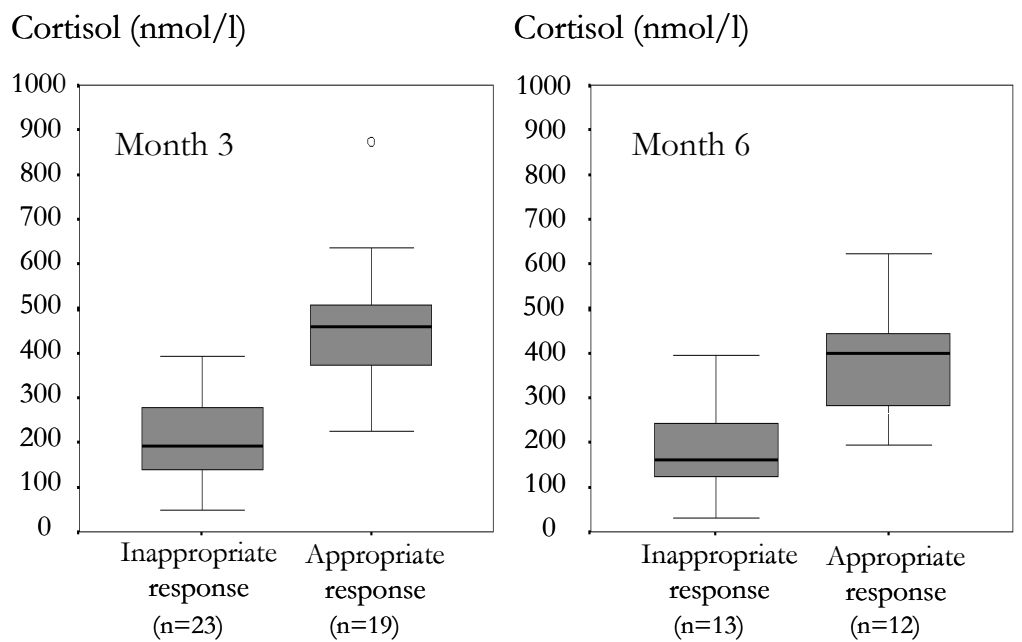

Figure 3.2 Baseline cortisol. The results after three months are presented in the left figure and the results after six months are presented in the right figure. The median and interquartile ranges are expressed in the boxes. The outliers are expressed in the T-bars. Extreme outliers are expressed separately $\left({ }^{\circ}\right)$.

\section{Symptoms of adrenal insufficiency}

Fourteen of the 15 patients who were randomized at month 3 to steroid withdrawal had their steroids withdrawn in two weeks. In the other patient prednisolone was reduced to $5 \mathrm{mg}$ due to prior severe rejection. None of these patients experienced clinical symptoms of adrenal insufficiency.

After six months, ten patients had their steroids withdrawn in a similar way. Five of them had an inappropriate response on the synacthen test. Three of these ten patients experienced symptoms that could be related to adrenal insufficiency: orthostatic hypotension $(\mathrm{n}=3)$ and weakness $(\mathrm{n}=1)$. These symptoms resolved after lowering anti-hypertensive drugs, increasing sodium-chloride intake, or spontaneously, respectively. No steroids were restarted. None of these patients experienced a stressful event in this period. The results of the SST of the symptomatic patients are presented in Table 3.3. The sensitivity of the SST at six months post-transplantation for symptomatic adrenal insufficiency after rapid steroid withdrawal was $67 \%$ and the specificity $57 \%$. The positive predictive value was $40 \%$ and the negative predictive value $80 \%$. 
Table 3.3. Test results of the symptomatic patients. The individual test results of the patients with symptoms that may result of adrenal insufficiency after six months of therapy. The response in patient 1 is appropriate in contrast to the response in patient 2 and $3\left({ }^{*}\right)$.

\begin{tabular}{lcccc}
\hline & \multicolumn{2}{c}{ Cortisol month $\mathbf{3}(\mathrm{nmol} / \mathbf{l})$} & \multicolumn{2}{c}{ Cortisol month 6 (nmol/1) } \\
Patient & baseline & stimulated & baseline & Stimulated \\
\hline 1 & 556 & 765 & 492 & 745 \\
2 & 393 & $478^{*}$ & 290 & $425^{*}$ \\
3 & 77 & $240^{*}$ & 135 & $296^{*}$ \\
\hline
\end{tabular}

Eleven patients had their steroids after six months post-transplantation gradually withdrawn in several months time. Six of them had an inappropriate response on the SST. They were well comparable to the patients with the rapid withdrawal, except for a larger baseline body mass index $\left(25.9 \pm \mathrm{kg} / \mathrm{m}^{2}\right.$ versus $23.4 \pm 1.3 \mathrm{~kg} / \mathrm{m}^{2}$, $P=0.01$, t-test). None of them experienced symptoms that could be attributed to adrenal insufficiency.

In the other four patients, the steroids were continued.

\section{Discussion}

This is the first study that prospectively evaluated the degree of suppression of the HPA-axis with $10 \mathrm{mg}$ prednisolone maintenance therapy from transplantation onwards. More than half of the patients had some degree of suppression after three or six months of therapy. Prolonging the duration of the maintenance therapy from three to six months also resulted in more suppression of the HPAaxis.

So far in renal transplantation, several studies regarding this issue have been performed in the last two decades ${ }^{8-13}$. However, almost all of these studies had a cross-sectional design and the dose of the steroids and the duration of the therapy were divers. Thus, the real degree of the problem remained unclear. Only one study evaluated adrenal suppression in a prospective way from transplantation onwards ${ }^{13}$. The dose of the steroids in that study, however, was two to three times higher than in our study.

In those studies, some degree of suppression of the HPA-axis was present in $43 \%{ }^{11}$ to $66 \%{ }^{12}$. However, due to differences in steroid dosages, duration of the therapy, and test methods, these data are incomparable.

One matter of debate with adrenal insufficiency is which test to perform for its detection. The insulin tolerance test (ITT) is considered to be the standard, because it tests the entire HPA-axis. However, its clinical use is remarkably 
restricted by its complexity and the risks obtained with inducing hypoglycemia. The synacthen test correlates well with the ITT ${ }^{14,15}$. Moreover, the SST is superior with regard to side effects, costs, and complexity. Thus, the synacthen test is a proper screening test for suppression of the HPA-axis.

Another point of controversy is the cut-off level by which patients are classified as having adrenal insufficiency. The data are derived from cortisol levels achieved by performing both an ITT and a SST and comparing the results of true patients to healthy volunteers ${ }^{14,16}$. In addition, the results obtained by the SST correlate well to cortisol levels measured during surgery ${ }^{17}$. The proposed stimulated cortisol cutoff level has been either $500 \mathrm{nmol} / 1^{16}, 550 \mathrm{nmol} / 1^{6,7}$, or even $600 \mathrm{nmol} / 1^{14,18}$. In patients with true adrenal insufficiency, in contrast to patients with suppression by external steroids, the test needs to be sensitive, because missing patients would be potentially dangerous in periods of stress, since these patients are totally dependent on external steroids in these circumstances. By applying these criteria, the number of patients with an inappropriate response in this study would range between 43\% (500 nmol/l after three months) and 64\% (600 nmol/1 after six months), Figure 3.3.

\section{Inappropriate response on synacthen}

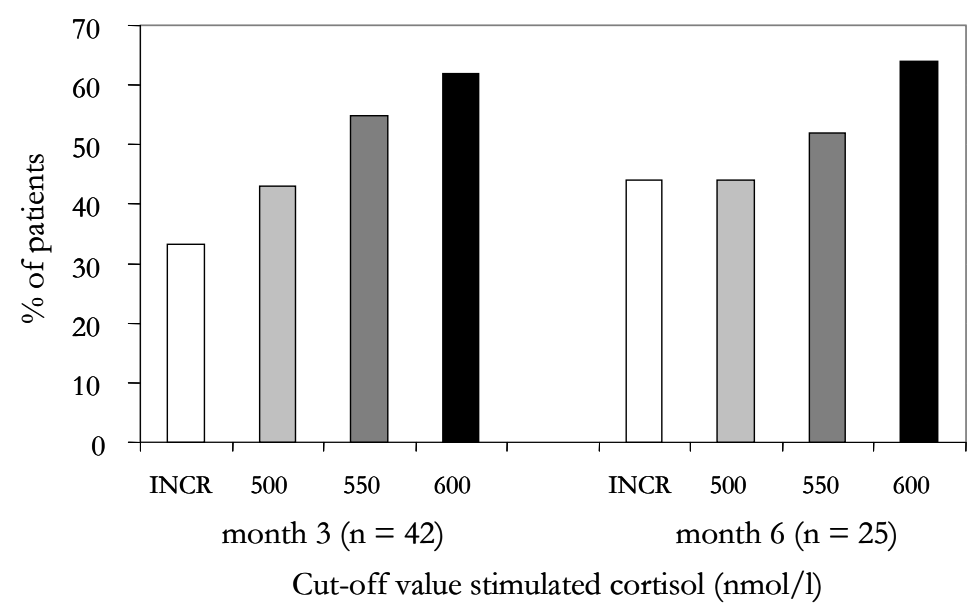

Figure 3.3 The number of patients with an inappropriate response on the short synacthen test. The response on the short synacthen test at different cut-off levels. INCR implies a stimulated cortisol of at least $550 \mathrm{nmol} / \mathrm{l}$ or a stimulated cortisol of $410 \mathrm{nmol} / \mathrm{l}$, provided that the increment was at least $137 \mathrm{nmol} / 1^{19}$. 
In former times, also the level of increment in serum cortisol was taken into account ${ }^{19}$. Because, the increment is dependent on the baseline value ${ }^{7}$, this criterion has been abandoned nowadays. When, a normal SST was defined by either a stimulated cortisol above $550 \mathrm{nmol} / 1$ or a stimulated cortisol of $410 \mathrm{nmol} / 1$, provided that the increase was at least $137 \mathrm{nmol} / 1^{19}$, the number of patients with an inappropriate response in this study would be reduced to $33 \%$ after three months and $44 \%$ after six months of therapy, Figure 3.3. These figures approximate more the incidence of symptoms of adrenal dysfunction observed in this study after rapid withdrawal of the steroids. In patients using steroids, the increment may indicate reversibility in the suppressed system and less risk of symptoms after rapid discontinuation of the steroids.

In the last decade, the $1 \mu \mathrm{g}$ low-dose synacthen test has been introduced as a more sensitive test ${ }^{14}$, because $1 \mu \mathrm{g}$ is about the lowest dose for maximal stimulation of the adrenal gland ${ }^{20}$. In patients with true adrenal insufficiency, in which it is potentially dangerous to miss patients with the disease, the low-dose SST may be of advantage. Since, there is already overestimation of the clinical situation with the conventional $0.25 \mathrm{mg} \mathrm{SST}$, this advantage is not attributable to adrenal suppression by external steroids. Furthermore, vials containing $1 \mu \mathrm{g}$ synacthen are not commercially available and preparing $1 \mu \mathrm{g}$ doses from the $0.25 \mathrm{mg}$ vials may be inaccurate.

A potential bias is that prednisolone cross-reacts with our method of detection of cortisol by $62 \%$. However, it is unlikely that this cross-reaction played a role, because the patients took their last dose at least 24 hours before the test and the plasma half-life of prednisolone is only three hours ${ }^{2}$. This implies that the next morning (after eight half-lives) maximally $4 \%$ of the original level still circulates.

In contrast to the plasma half-life, the duration of the biological effects of the steroids is much longer and for prednisolone, the biological half-life varies between 12 and 36 hours $^{2}$. This explains that patients have suppression of the HPA-axis when measured 24 hours after the last dose.

The fact that not all patients have suppression of the HPA-axis implies that there is a large inter-individual susceptibility. This is in agreement with previous studies ${ }^{15,21,22}$. We evaluated our data to look for determinants that account for this variation. By multivariate analysis, none of the studied potential influencing factors had a significant effect on the response of the SST and we could not confirm the observed higher response on the SST of females compared to males ${ }^{23}$. On the other hand, both age and body mass index, but not gender, were significantly related to the baseline cortisol level after six months of therapy. Patients with a higher age showed more suppression of the baseline cortisol and 
patients with a higher body mass index showed less suppression of the baseline cortisol.

There has been controversy whether the duration of the steroid therapy has an influence on the degree of suppression of the HPA-axis. One cross-sectional study showed a correlation between these factors ${ }^{15}$, whereas two other studies didn't ${ }^{21,22}$. This prospective study shows that prolonging the duration of the steroid therapy results in more suppression of the HPA-axis.

All patients with a baseline cortisol level below $100 \mathrm{nmol} / \mathrm{l}$ had an inappropriate response (Figure 3.2). This is in agreement with previous studies ${ }^{4,5}$. When analysing the HPA-axis, these patients can refrain from testing. In addition, only one patient out of nine (11\%) with a baseline cortisol below $200 \mathrm{nmol} / \mathrm{l}$ after six months had a normal response. Also, all patients with a baseline cortisol above $400 \mathrm{nmol} / 1$ had a normal response and can also refrain from testing (Figure 3.2). This is comparable to the results of Pavord et al. ${ }^{5}$ and Hurel et al. ${ }^{18}$. Hägg et al. ${ }^{4}$ advized a baseline cortisol above $300 \mathrm{nmol} / 1$ to be consistent with a normal response. In our study, three out of 20 patients $(15 \%)$ after three months and one patient out of ten $(10 \%)$ after six months would have been falsely classified as appropriate when using the criterion of Hägg.

Costs can be reduced by applying a two step analysis of first a baseline cortisol and secondly, when the baseline cortisol ranges between 200 and $400 \mathrm{nmol} / \mathrm{l}$, a stimulated sample 30 minutes after synacthen. In that case, only $40 \%$ of the patients, both after three and after six months, needed an evaluation after synacthen.

Although the evaluation of the magnitude of the suppression of the HPA-axis was the primary goal of this study, a second goal was the identification of a tool to detect which patients may especially be at risk. We observed that clinical symptoms, possibly related to steroid withdrawal were uncommon, despite the considerable proportion of patients with some degree of suppression of the HPAaxis. In this study, none of the patients experienced symptoms after three months of therapy, while three out of ten patients experienced symptoms after six months of therapy. These symptoms were mild and resolved without reintroduction of the steroids. However, none of our patients came in a "stressful" situation. Thus, this study reveals no data about the risk of two weeks steroid withdrawal in or just before a stressful situation. In the past, abrupt stopping of steroids caused lifethreatening conditions. The reliability for prediction of symptomatic adrenal insufficiency by the SST appeared poor with a positive predictive value of only $40 \%$. The negative predictive value was higher with $80 \%$.

Furthermore, clinical symptoms of adrenal insufficiency are non-specific. Steroid withdrawal has been shown to lower blood pressure ${ }^{24}$ and the orthostatic 
complaints of our patients could also be the result of the blood pressure lowering effect of the steroid withdrawal only. Besides orthostatic complaints, one patient also experienced weakness. Because this patient had also a suppressed baseline cortisol level and a clear abnormal response (patient 3; Table 3.3), the clinical symptoms in this patient probably resulted from true adrenal insufficiency. Considering this patient as the only true symptomatic patient, the positive predictive value would drop to $20 \%$, but the negative predictive value would improve to $100 \%$.

Thus, the SST overestimates the risk of clinical symptoms of adrenal insufficiency after rapid steroid withdrawal, but it can reasonably detect patients, in which it is definitely a safe procedure. This is in agreement with the data obtained by Bromberg et al. ${ }^{10,11}$.

In rejectors, who had been administered high doses of methylprednisolone, more suppression of the HPA-axis was observed. With the low number of rejectors, this did not reach statistical significance. The rejectors may especially be at risk for adrenal insufficiency when steroids are rapidly withdrawn. However, these patients normally don't belong to a population scheduled for such a procedure.

In summary, suppression of the HPA-axis is common in renal transplantation with $10 \mathrm{mg}$ prednisolone maintenance dose and exists in more than half of the patients. The degree of suppression increases from three to six months after transplantation. Despite this suppression, it appeared safe to withdraw steroids in two weeks after three months of therapy. After six months of therapy, however, this rapid discontinuation resulted in clinical symptoms, although serious problems did not occur. Since no patient experienced a stressful event, this study reveals no data about the safety of rapid steroid withdrawal in periods of stress. Patients with a baseline cortisol below $200 \mathrm{nmol} / \mathrm{l}$ or above $400 \mathrm{nmol} / \mathrm{l}$ can reasonably be classified as having an inappropriate respectively appropriate response on the short synacthen test and refrain from testing. The short synacthen test has a poor predictive value for clinical symptoms after rapid steroid withdrawal, but, in patients with a normal test, rapid discontinuation is reasonably safe. 


\section{References}

1. Spiegel RJ, Vigersky RA, Oliff AI, Echelberger CK, Bruton J and Poplack DG. Adrenal suppression after short-term corticosteroid therapy. Lancet 1979;1:630-3.

2. Walsh JP and Dayan CM. Role of biochemical assessment in management of corticosteroid withdrawal. Ann Clin Biochem 2000;37:279-88.

3. Racusen LC, Solez K, Colvin RB, Bonsib SM, Castro MC, Cavallo T, Croker BP, Demetris AJ, Drachenberg CB, Fogo AB, Furness P, Gaber LW, Gibson IW, Glotz D, Goldberg JC, Grande JG, Halloran PF, Hansen HE, Hartley B, Hayry PJ, Hill CM, Hoffman EO, Hunsicker LG, Lindblad AS, Marcussen N, Mihatsch MJ, Nadasdy T, Nickerson P, Olsen TS, Papadimitriou JC, Randhawa PS, Rayner DC, Roberts I, Rose S, Rush D, Salinas-Madrigal L, Salomon DR, Sund S, Taskinen E, Trpkov K and Yamaguchi Y. The Banff 97 working classification of renal allograft pathology. Kidney Int 1999;55:713-23.

4. Hägg E, Asplund K and Lithner F. Value of basal plasma cortisol assays in the assessment of pituitary-adrenal insufficiency. Clin Endocrinol 1987;26:221-6.

5. Pavord SR, Girach A, Price DE, Absalom SR, Falconer-Smith J and Howlett TA. A retrospective audit of the combined pituitary function test, using the insulin stress test, TRH and GnRH in a district laboratory. Clin Endocrinol 1992;36:135-9.

6. Oelkers W. Adrenal insufficiency. N Engl J Med 1996;335:1206-12.

7. May ME and Carey RM. Rapid adrenocorticotropic hormone test in practice. Retrospective review. Am J Med 1985;79:679-84.

8. Canafax DM, Mann HJ, Sutherland DER, Simmons RL and Najarian JS. The use of a cosyntropin stimulation test to predict adrenal suppression in renal transplant patients being withdrawn from prednisone. Transplantation 1983;36:143-6.

9. Shapiro R, Carroll PB, Tzakis AG, Cemaj S, Lopatin WB and Nakazato P. Adrenal reserve in renal transplant recipients with cyclosporin, azathioprine, and prednisone immunosuppression. Transplantation 1990;49:1011-3.

10. Bromberg JS, Alfrey EJ, Barker CF, Chavin KD, Dafoe DC, Holland T, Naji A, Perloff LJ, Zellers LA and Grossman RA. Adrenal suppression and steroid supplementation in renal transplant recipients. Transplantation 1991;51:385-90.

11. Bromberg JS, Baliga P, Cofer JB, Rajagopalan PR and Friedman RJ. Stress steroids are not required for patients receiving a renal allograft and undergoing operation. J Am Coll Surg 1995; 180:532-6.

12. Rodger RSC, Watson MJ, Sellars L, Wilkinson R, Ward MK and Kerr DNS. Hypothalamicpituitary-adrenocortical suppression and recovery in renal transplant patients returning to maintenance dialysis. Q J Med 1986;61:1039-46.

13. Oka K, Shimodaira H, Hirano T, Sakurai E, Tamaki T and Kozaki M. Comparison of adrenal functions in kidney transplant recipients with different long-term immunosuppressive treatments - prednisolone and azathioprine versus prednisolone and cyclosporine. Transplantation 1993;56:603-9. 
14. Abdu TAM, Elhadd TA, Neary R and Clayton RN. Comparison of the low dose short synacthen test $(1 \mu \mathrm{g})$, the conventional dose short synacthen test $(250 \mu \mathrm{g})$, and the insulin tolerance test for assessment of the hypothalamo-piyuitary-adrenal axis in patients with pituitary disease. J Clin Endocrinol Metab 1999;84:838-43.

15. Kane KF, Emery P, Sheppard MC and Stewart PM. Assessing the hypothalamo-pituitaryadrenal axis in patients on long-term glucocorticoid therapy: the short synacthen versus the insulin tolerance test. Q J Med 1995;88:263-7.

16. Lindholm $\mathrm{J}$ and Kehlet H. Re-evaluation of the clinical value of the $30 \mathrm{~min}$ ACTH test in assessing the hypothalamic-pituitary-adrenocortical function. Clin Endocrinol 1987;26:53-9.

17. Kehlet $\mathrm{H}$ and Binder $\mathrm{C}$. Value of an ACTH test in assessing hypothalamic-pituitaryadrenocortical function in glucocorticoid-treated patients. Br Med J 1973;2:147-9.

18. Hurel SJ, Thompson CJ, Watson MJ, Harris MM, Baylis PH and Kendall-Taylor P. The short synacthen and insulin stress tests in the assessment of the hypothalamic-pituitary-adrenal axis. Clin Endocrinol 1996;44:141-6.

19. Tyrrell JB, Aron DC and Forsham PH. Glucocorticoids and adrenal androgens. In: Greenspan FS. Basic and Clinical Endocrinology. third ed. Appleton \& Lange, East Norwalk, Connecticut, 1991:323-62.

20. Crowley S, Hindmarsh PC, Holownia P, Honour JW and Brook CGD. The use of low doses of ACTH in the investigation of adrenal function in man. J Endocrinol 1991;130:475-9.

21. LaRochelle GE jr, LaRochelle AG, Ratner RE and Borenstein DG. Recovery of the hypothalamic-pituitary-adrenal (HPA) axis in patients with rheumatic diseases receiving lowdose prednisone. Am J Med 1993;95:258-64.

22. Schlaghecke R, Kornely E, Santen RTh and Ridderskamp P. The effect of long-term glucocorticoid therapy on pituitary-adrenal responses to exogenous corticotropin-releasing hormone. N Engl J Med 1992;326:226-30.

23. Clark PM, Neylon I, Raggatt PR, Sheppard MC and Stewart PM. Defining the normal cortisol response to the short Synacthen test: implications for the investigation of hypothalamicpituitary disorders. Clin Endocrinol 1998;49:287-92.

24. Ahsan N, Hricik D, Matas A, Rose S, Tomlanovich S, Wilkinson A, Ewell M, McIntosh M, Stablein D, Hodge E and for the Steroid Withdrawal Group. Prednisone withdrawal in kidney transplant recipients on cyclosporin and mycophenolate mofetil - a prospective randomized study. Transplantation 1999;68:1865-74. 


\section{Chapter 4}

\section{Increase in tacrolimus trough levels after steroid withdrawal}

EM van Duijnhoven, JMM Boots, MHL Christiaans, LML Stolk, NA Undre, JP van Hooff

Transplant International 2003;16(10):721-725 


\section{Abstract}

Although there are experimental reports of cytochrome P450 3A4 iso-enzyme (CYP3A4) induction by glucocorticoids, there are no clinical reports about an interaction between tacrolimus and steroids. Therefore, tacrolimus trough level and dose were compared after dose-normalization before and after withdrawal of prednisolone. After withdrawal of $5 \mathrm{mg}$ prednisolone, median tacrolimus dosenormalized level increased $14 \%$ in the retrospective ( $\mathrm{n}=54)$, and $11 \%$ in the prospective $(n=8)$ part of the study. After withdrawal of $10 \mathrm{mg}$, this was $33 \%$ $(\mathrm{n}=30)$ and $36 \%(\mathrm{n}=14)$, respectively. An additional pharmacokinetic part of the study $(n=8)$ revealed an $18 \%$ increase in AUC $(P=0.05)$ after withdrawal of $5 \mathrm{mg}$ prednisolone, compatible with a reduced metabolism after steroid withdrawal. The significant increase in tacrolimus exposure after steroid withdrawal may on the one hand counteract the reduction in immunosuppression intended by steroid withdrawal, and on the other hand may result in an increase of serum creatinine which could be misinterpreted as rejection! 


\section{Introduction}

Tacrolimus is an effective drug for the prevention of acute rejection after renal transplantation ${ }^{1-4}$. Generally, it is combined with steroids, both initially and during maintenance therapy. The Pittsburgh group and other investigators ${ }^{5-9}$ have shown that steroids can be withdrawn after renal transplantation in a large proportion of patients. In our center as well, steroids were withdrawn, without rejection, in a majority of patients, who had been treated with tacrolimus and steroids for more than three to six months, had stable graft function and no proteinuria ${ }^{10}$. In several of these patients, we observed an increase in tacrolimus trough levels following steroid withdrawal, although the tacrolimus dose was unchanged.

An interaction between steroids and tacrolimus has been described in in vitro studies and in in vivo animal studies ${ }^{11-15}$. Tacrolimus is metabolized by the cytochrome P450 3A4 (CYP3A4) iso-enzyme. Glucocorticoids are inducers of CYP3A4 and consequently would be expected to increase the metabolism of tacrolimus ${ }^{16}$.

P-glycoprotein is responsible for an efflux of tacrolimus from the enterocytes back into the gut lumen. It is so far unclear whether interaction on the Pglycoprotein (P-gp) level could also play a role ${ }^{17}$.

Since increase in systemic exposure to tacrolimus after steroid withdrawal has not yet been described, we studied the effect of withdrawal of two different steroid dosages on the systemic exposure to tacrolimus. The evaluations were made both by retrospective and prospective examination of tacrolimus trough level and corresponding dosing data as well as by a pharmacokinetic study.

\section{Patients and methods}

\section{Patients}

Renal allograft recipients on tacrolimus-based immunosuppression, at least three months post-transplantation were included. Patients with a history of acute rejection (BANFF 2 or higher), steroid resistant rejection, hyperimmunized state, unstable renal function or proteinuria, had been excluded from steroid withdrawal. In the retrospective part of the study all patients with steroid withdrawal who met these criteria were evaluated. In 54 Caucasian patients $5 \mathrm{mg}$ prednisolone per day had been stopped, and in 30 Caucasian patients $10 \mathrm{mg}$ prednisolone per day. Patients with changes in co-medication were excluded. Patients with trough levels collected less than 11 or more than 13 hours after the last tacrolimus ingestion were excluded. In the prospective part of the study, eight Caucasian patients were evaluated before and after withdrawal of $5 \mathrm{mg}$ prednisolone, and 14 Caucasian before and after withdrawal of $10 \mathrm{mg}$ prednisolone. Additionally, in eight patients pharmacokinetic parameters were prospectively compared before and after 
withdrawal of $5 \mathrm{mg}$ of prednisolone, while tacrolimus dose did not change. Changes in co-medication were not allowed. Trough levels were taken between 11.45 and 12.15 hours after the last tacrolimus ingestion.

All patients had given informed consent. The studies were approved by the ethical committee of the hospital.

Patient characteristics are shown in Table 4.1.

Table 4.1 Basic patient characteristics

\begin{tabular}{lcccc}
\hline & \multicolumn{2}{c}{ Retrospective part } & \multicolumn{2}{c}{ Prospective part } \\
\hline Steroid dose (mg) & $5 \mathrm{mg}$ & $10 \mathrm{mg}$ & $10 \mathrm{mg}$ & $5 \mathrm{mg}$ \\
Number of patients & 54 & 30 & 14 & 8 \\
Gender: male/female & $34 / 20$ & $23 / 7$ & $10 / 4$ & $7 / 1$ \\
Tx number: first/retransplant & $44 / 12$ & $25 / 5$ & $13 / 1$ & $6 / 2$ \\
Tx type: LRD/LUD/cadaveric & $0 / 6 / 48$ & $1 / 2 / 27$ & $1 / 1 / 12$ & $0 / 1 / 7$ \\
Immunosupp: AZA/MMF/none & $8 / 4 / 44$ & $0 / 13 / 17$ & $0 / 14 / 0$ & $0 / 1 / 7$ \\
Diabetes mellitus & 4 & 5 & 1 & 0 \\
Age (years) & $51.5(15.9-67.5)$ & $57.0(22.0-72.0)$ & $60.5(32.0-75.0)$ & $50.9(32.7-67.8)$ \\
Body mass index $\left(\mathrm{kg} / \mathrm{m}^{2}\right)$ & $24.7(16.6-36.5)$ & $24.5(15.6-31.3)$ & $25.2(17.1-31.7)$ & $25.8(18.8-34.7)$ \\
Time after tx (days) & $262(88-1626)$ & $242(127-393)$ & $90(85-92)$ & $209(151-864)$ \\
\hline
\end{tabular}

LRD=living related donor, LUD=living unrelated donor. Immunosupp: other immunosuppressive agents. Data are given as numbers or median (+ range).

\section{Retrospective evaluations}

Steady-state tacrolimus whole blood 12-hour trough levels and the corresponding daily doses were recorded before tapering of steroids and after complete cessation of steroids. Steady state was assumed when the daily dose of tacrolimus was stable (unchanged) for three or more days prior to the measurement of trough level. Blood levels taken shortly before the start of steroid reduction, and those taken shortly after complete cessation of steroids that met the steady-state definition were used for this study. To account for changes in tacrolimus dose, comparisons were made between the dose-normalized concentrations (tacrolimus trough level divided by the corresponding daily dose).

\section{Prospective evaluations}

In the patients using $10 \mathrm{mg}$ prednisolone, after obtaining normal ACTH stimulation tests, prednisolone daily dose was reduced from $10 \mathrm{mg}$ to $5 \mathrm{mg}$ for one week and $2.5 \mathrm{mg}$ for the next week and thereafter completely stopped. Tacrolimus doses and tacrolimus trough levels that met the steady-state definition, and serum creatinine levels were evaluated at the same time at the start of steroid withdrawal and after complete cessation of steroids. 
In the patients using $5 \mathrm{mg}$ prednisolone, 209 (151-864) days after transplantation prednisolone was tapered to $2.5 \mathrm{mg} / \mathrm{day}$ for one week and thereafter completely stopped.

Immediately before, and one week after complete cessation of steroids, tacrolimus blood concentration-time profiles were taken over a 12 hour dosing period. Blood samples were taken at pre-dose $(0)$ and then at $0.25,0.5,0.75,1.0,2.0,3.0,4.0,5.0$, 7.5, 9.0 and 12.0 hours post-dose. For a period of one-week prior to the first pharmacokinetic profile until after the second profile, the daily dose of tacrolimus had to be unchanged. Tacrolimus was administered after an overnight fast, and a standard breakfast containing $1928 \mathrm{~kJ}$ of energy, 43\% fat content, was given after the withdrawal of 1-hour blood.

Concentrations of tacrolimus in whole blood were determined by IMx (Abbott, Hoofddorp, The Netherlands). Blood concentration-time data were used to calculate standard pharmacokinetic parameters $\left(\mathrm{C}_{\max }, \mathrm{t}_{\max }\right.$, oral clearance and AUC $_{0-12}$, using the computer program MWPHARM 3.30 (Mediware, Groningen, The Netherlands)).

\section{Statistics}

For statistical analysis, SPSS version 10.0 for Windows (Cary Inc, Chicago, Il) was used. To compare dose-normalized level and pharmacokinetic parameters before and after steroid withdrawal, Wilcoxon matched-pairs signed rank sum test was performed. Unless indicated otherwise, data are given as median and range. A Pvalue below 0.05 was considered to be statistically significant.

\section{Results}

In Table 4.2 are presented tacrolimus dose, trough level and dose-normalized level before and after steroid withdrawal.

In the retrospective part of the study steroid tapering started at a median of 262 (range 88-1626) days after transplantation, and was completed in 100 (0-208) days in patients using $5 \mathrm{mg}$ prednisolone per day. In patients using $10 \mathrm{mg}$ prednisolone per day steroid tapering started 242 (127-393) days after transplantation, and was completed in 68 (6-311) days. Dose-normalized level increased by $14 \%$ after withdrawal of $5 \mathrm{mg}$ prednisolone, from 1.4 to $1.6 \mathrm{ng} / \mathrm{ml} \mathrm{per} \mathrm{mg}(P=0.003)$, and by $33 \%$ after withdrawal of $10 \mathrm{mg}$ prednisolone, from 1.5 to $2.0 \mathrm{ng} / \mathrm{ml}$ per $\mathrm{mg}$ $(P=0.011)$. 
Table 4.2 Tacrolimus dose and trough level before and after steroid withdrawal

\begin{tabular}{|c|c|c|c|c|c|}
\hline & & & $\begin{array}{c}\text { Before steroid } \\
\text { withdrawal }\end{array}$ & $\begin{array}{l}\text { After steroid } \\
\text { withdrawal }\end{array}$ & $P$ \\
\hline \multirow[t]{8}{*}{ Retrospective } & $5 \mathrm{mg}$ prednisolone & $\begin{array}{l}\text { trough level } \\
(\mathrm{ng} / \mathrm{ml})\end{array}$ & $8.5 \quad(4.3-17.2)$ & $8.3 \quad(3.6-25.9)$ & \\
\hline & $\mathrm{n}=54$ & dose & $6.0(2.0-20.0)$ & $5.0 \quad(2.0-19.0)$ & \\
\hline & & $(\mathrm{mg} / \mathrm{d})$ & & & \\
\hline & & $\begin{array}{l}\text { dose-normalized level } \\
\text { (ng/ml per } \mathrm{mg} / \mathrm{d})\end{array}$ & $1.4 \quad(0.5-4.3)$ & $1.6 \quad(0.6-7.8)$ & 0.003 \\
\hline & $10 \mathrm{mg}$ prednisolone & $\begin{array}{l}\text { trough level } \\
(\mathrm{ng} / \mathrm{ml})\end{array}$ & $7.8 \quad(4.2-25.6)$ & $9.4 \quad(5.3-16.8)$ & \\
\hline & $\mathrm{n}=30$ & dose & $5.0 \quad(2.0-14.0)$ & $4.5 \quad(2.0-14.0)$ & \\
\hline & & $(\mathrm{mg} / \mathrm{d})$ & & & \\
\hline & & $\begin{array}{l}\text { dose-normalized level } \\
\text { (ng/ml per } \mathrm{mg} / \mathrm{d})\end{array}$ & $1.5 \quad(0.5-6.4)$ & $2.0 \quad(0.6-4.9)$ & 0.011 \\
\hline \multirow[t]{6}{*}{ Prospective } & $10 \mathrm{mg}$ prednisolone & $\begin{array}{l}\text { trough level } \\
(\mathrm{ng} / \mathrm{ml})\end{array}$ & $8.3 \quad(4.9-14.5)$ & $11.9(7.6-15.0)$ & \\
\hline & $\mathrm{n}=14$ & $\begin{array}{l}\text { Dose } \\
(\mathrm{mg} / \mathrm{d})\end{array}$ & $5.5(3.0-15.0)$ & $5.5(4.0-16.0)$ & \\
\hline & & $\begin{array}{l}\text { dose-normalized level } \\
\text { (ng/ml per } \mathrm{mg} / \mathrm{d})\end{array}$ & $1.4 \quad(0.2-2.9)$ & $1.9 \quad(0.8-3.5)$ & 0.002 \\
\hline & $5 \mathrm{mg}$ prednisolone & $\begin{array}{l}\text { trough level } \\
(\mathrm{ng} / \mathrm{ml})\end{array}$ & $7.3 \quad(3.8-9.9)$ & $8.1 \quad(5.0-14.1)$ & \\
\hline & $\mathrm{n}=8$ & $\begin{array}{l}\text { Dose } \\
(\mathrm{mg} / \mathrm{d})\end{array}$ & $5.0 \quad(2.5-13.0)$ & $5.0 \quad(2.5-13.0)$ & \\
\hline & & $\begin{array}{l}\text { dose-normalized level } \\
\text { (ng/ml per } \mathrm{mg} / \mathrm{d})\end{array}$ & $1.6 \quad(0.4-4.0)$ & $1.8 \quad(0.6-5.6)$ & 0.058 \\
\hline
\end{tabular}

Median and ranges of tacrolimus trough levels, tacrolimus dose and dose adjusted level before and after steroid withdrawal

In the prospective part of the study, 14 patients were evaluated before and 17 (13-31) days after withdrawal of $10 \mathrm{mg}$ prednisolone, 90 (85-92) days after transplantation, and eight patients seven days after withdrawal of $5 \mathrm{mg}$ prednisolone, 209 (151-864) days after transplantation. Dose-normalized level increased by $36 \%$ after withdrawal of $10 \mathrm{mg}$ prednisolone, from 1.4 to $1.9 \mathrm{ng} / \mathrm{ml}$ per $\mathrm{mg}(P=0.002)$, and by $12 \%$ after withdrawal of $5 \mathrm{mg}$ prednisolone, from 1.6 to $1.8 \mathrm{ng} / \mathrm{ml}$ per $\mathrm{mg}(P=0.058)$. After withdrawal of $10 \mathrm{mg}$ prednisolone, serum creatinine increased from $143(67-246) \mu \mathrm{mol} / 1$ to $151(67-259) \mu \mathrm{mol} / 1(P=$ $0.034)$.

Figures 4.1 and 4.2 show relative changes in tacrolimus dose-normalized levels after withdrawal of 5 and $10 \mathrm{mg}$ prednisolone in all patients. Increases of more than $20 \%$ occurred in $43 \%$ of the patients after withdrawal of $5 \mathrm{mg}$, and in $61 \%$ after withdrawal of $10 \mathrm{mg}$ prednisolone. 


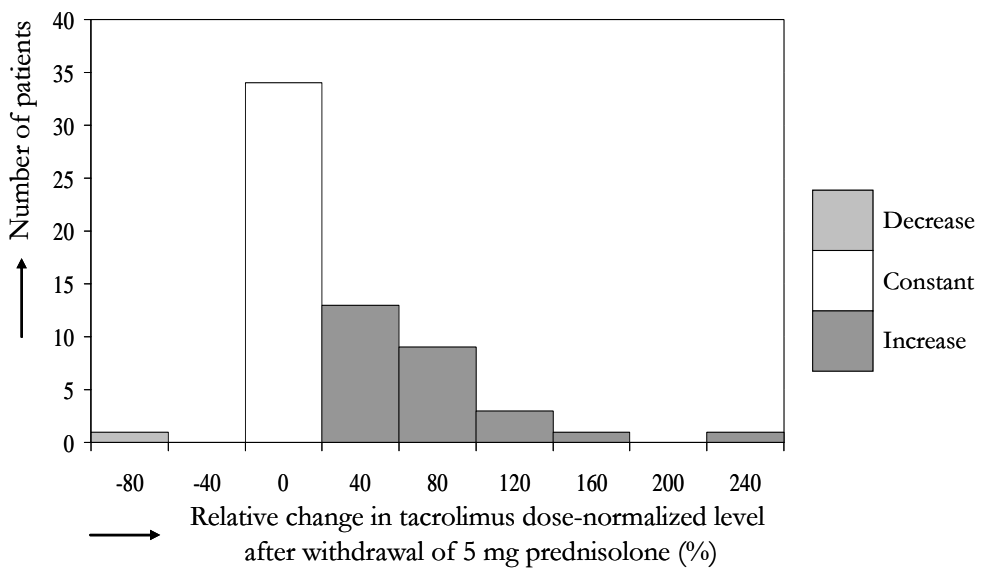

Figure 4.1 Relative changes (\%) in tacrolimus dose-normalized level after withdrawal of $5 \mathrm{mg}$ prednisolone for all retrospective and prospective patients $(\mathrm{n}=62)$.

Tacrolimus dose-normalized level was defined as tacrolimus trough level divided by corresponding daily dose. A decrease in tacrolimus dose-normalized level was defined as a reduction of tacrolimus dose-normalized level of $20 \%$ or more, and an increase as an addition of $20 \%$ or more, when tacrolimus dose-normalized level had changed less than $20 \%$, this was regarded as no change.

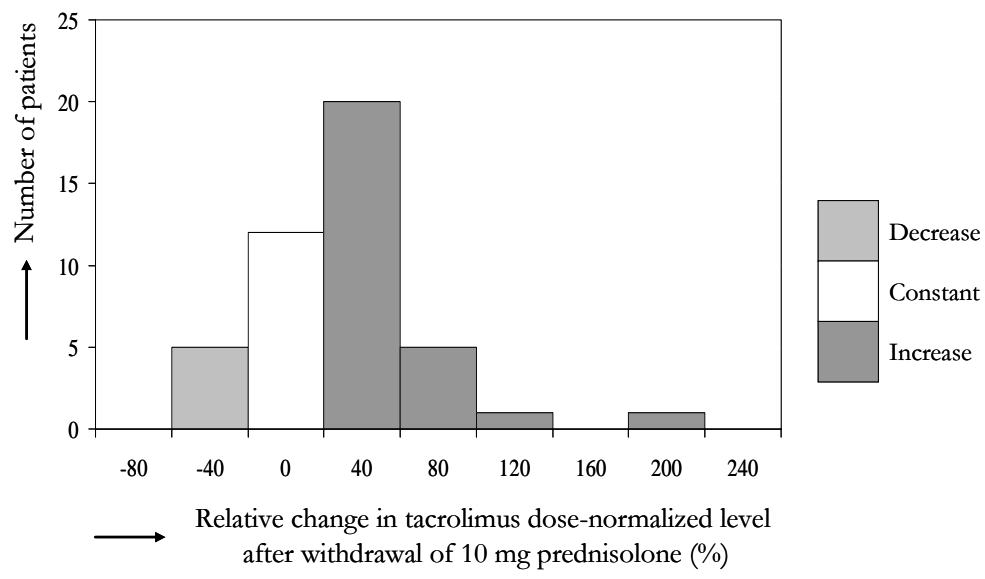

Figure 4.2 Relative changes (\%) in tacrolimus dose-normalized level after withdrawal of $10 \mathrm{mg}$ prednisolone for all retrospective and prospective patients $(\mathrm{n}=44)$.

Tacrolimus dose-normalized level was defined as tacrolimus trough level divided by corresponding daily dose. A decrease in tacrolimus dose-normalized level was defined as a reduction of tacrolimus dose-normalized level of $20 \%$ or more, and an increase as an addition of $20 \%$ or more, when tacrolimus dose-normalized level had changed less than $20 \%$, this was regarded as no change. 
Table 4.3 shows the pharmacokinetic parameters before and after steroid withdrawal.

The median AUC increased from $132.3 \mathrm{ng} \cdot \mathrm{h} / \mathrm{ml}$ before steroid withdrawal to $156.6 \mathrm{ng} . \mathrm{h} / \mathrm{mL}$ after steroid withdrawal $(P=0.05)$. The corresponding values for oral clearance were $0.280 \mathrm{l} / \mathrm{h} / \mathrm{kg}$ and $0.253 \mathrm{l} / \mathrm{h} / \mathrm{kg}(P=0.05)$, trough levels were $7.3 \mathrm{ng} / \mathrm{ml}$ and $8.1 \mathrm{ng} / \mathrm{ml}(P=0.08)$ and $C_{\max }$ were $24.9 \mathrm{ng} / \mathrm{ml}$ and $27.1 \mathrm{ng} / \mathrm{ml}$, respectively $(P=0.12)$.

Table 4.3Pharmacokinetic parameters before and after steroid withdrawal for each patient

\begin{tabular}{|c|c|c|c|c|c|c|c|c|c|c|}
\hline & \multicolumn{2}{|c|}{$\begin{array}{c}\text { Trough level } \\
(\mathrm{ng} / \mathrm{ml})\end{array}$} & \multicolumn{2}{|c|}{$\mathrm{C}_{\max }(\mathrm{ng} / \mathrm{ml})$} & \multicolumn{2}{|c|}{$\mathbf{T}_{\max }$ (hours) } & \multicolumn{2}{|c|}{$\begin{array}{c}\text { Oral clearance } \\
(1 / \mathrm{h} / \mathrm{kg})\end{array}$} & \multicolumn{2}{|c|}{$\operatorname{AUC~(ng.h/ml)~}$} \\
\hline & pre & post & pre & post & pre & post & pre & post & pre & post \\
\hline Patient 1 & 8.3 & 7.9 & 22.5 & 26.9 & 1.00 & 1.00 & 0.293 & 0.238 & 135.1 & 166.8 \\
\hline Patient 2 & 9.2 & 8.0 & 28.2 & 27.3 & 1.00 & 1.00 & 0.291 & 0.338 & 170.1 & 146.4 \\
\hline Patient 3 & 4.6 & 5.0 & 16.0 & 12.2 & 1.00 & 1.00 & 0.251 & 0.267 & 99.1 & 94.1 \\
\hline Patient 4 & 6.3 & 6.9 & 19.6 & 23.7 & 0.45 & 1.00 & 0.268 & 0.178 & 94.5 & 140.8 \\
\hline Patient 5 & 9.0 & 10.4 & 30.9 & 42.3 & 1.00 & 1.00 & 0.206 & 0.188 & 185.2 & 201.9 \\
\hline Patient 6 & 3.8 & 8.4 & 36.9 & 37.8 & 1.00 & 1.00 & 0.413 & 0.269 & 129.6 & 184.0 \\
\hline Patient 7 & 9.9 & 14.1 & 27.3 & 29.8 & 1.00 & 1.00 & 0.133 & 0.099 & 175.4 & 237.8 \\
\hline Patient 8 & 5.4 & 8.1 & 15.6 & 19.2 & 2.00 & 2.00 & 0.619 & 0.584 & 103.3 & 136.6 \\
\hline Median & 7.3 & 8.1 & 24.9 & 27.1 & 1.00 & 1.00 & 0.280 & 0.253 & 132.3 & 156.6 \\
\hline$P$ & \multicolumn{2}{|c|}{0.08} & \multicolumn{2}{|c|}{0.12} & \multicolumn{2}{|c|}{0.32} & \multicolumn{2}{|c|}{0.05} & \multicolumn{2}{|c|}{0.05} \\
\hline
\end{tabular}

Tacrolimus trough level, $\mathrm{C}_{\max }$ (the maximum level after tacrolimus ingestion), $\mathrm{T}_{\max }$ (the time after transplantation when the maximum level was reached), oral clearance and area under the curve (total tacrolimus exposure)

\section{Discussion}

Based on our clinical observations, and supported by reports about a possible interaction between tacrolimus and steroids ${ }^{11-17}$, we evaluated the effect of steroid withdrawal on the systemic exposure to tacrolimus.

The results of our evaluations have confirmed that the withdrawal of steroids results in an increased systemic exposure to tacrolimus. The increase in systemic exposure to tacrolimus following the withdrawal of $10 \mathrm{mg}$ prednisolone was higher (33-36\%) when compared with the increase after withdrawal of $5 \mathrm{mg}$ prednisolone (12-14\%). The increase in AUC that is observed is not associated with an increase in either $\mathrm{C}_{\max }$ or $\mathrm{t}_{\max }$, suggesting that the processes of absorption remain unaffected. Therefore, a role of P-glycoprotein seems less likely. The most likely cause for the increased exposure following steroid withdrawal may be associated with decrease in the metabolic clearance as a consequence of the reversal of CYP3A4 induction by steroids. 
In an earlier study we described that oral clearance decreases over time ${ }^{18}$. From the current study, we can conclude that, besides increasing hemoglobin and albumin levels, also the marked steroid reduction in this period explains the decrease in oral clearance over time. Furthermore, the current study suggests that the effect of steroid withdrawal is steroid dose dependent. After withdrawal of only $10 \mathrm{mg}$ prednisolone, an increase in tacrolimus dose-normalized ratio of $\pm 35 \%$ was found. Generally, much higher steroid dosages are used in renal transplantation. After withdrawal of such higher steroid dosages, an even larger increase in tacrolimus trough levels might be expected.

The assay method used to determine tacrolimus blood (Imx, Abbott) is reported to have a precision of up to $\pm 20 \%$ at $5 \mathrm{ng} / \mathrm{ml}^{19,20}$. Therefore, an increase in blood level of $>20 \%$ was considered to be relevant. Such an increase occurred in $43 \%$ of the patients after withdrawal of $5 \mathrm{mg}$ prednisolone, and $61 \%$ after withdrawal of $10 \mathrm{mg}$ prednisolone. In individual patients the increase amounted to as much as $200 \%$. This indicates that the increase in tacrolimus trough level after steroid withdrawal is a clinically important issue.

The increase in systemic exposure to tacrolimus after steroid withdrawal may on one hand counteract the reduction in immunosuppression intended by steroid withdrawal, and on the other hand will in some patients result in an increase of serum creatinine. This was observed in an earlier study ${ }^{21}$, where we found a significant increase in creatinine clearance by $16 \%$ after a $33 \%$ reduction of tacrolimus levels, from $9.5 \mathrm{ng} / \mathrm{ml}$ to $6.4 \mathrm{ng} / \mathrm{ml}$, and was confirmed in this study. Such an increase of creatinine levels due to an increase in tacrolimus trough levels after steroid withdrawal could be misinterpreted as rejection! Therefore, evaluation of tacrolimus trough level within one to two weeks after steroid withdrawal is advisable for all patients. Tacrolimus dose should be reduced in all patients with a significant increase in trough level after steroid withdrawal. This is obviously also financially beneficial. 


\section{References}

1. Johnson C, Ahsan N, Gonwa T, Halloran P, Stegall M, Hardy M, Metzger R, Shield III C, Rocher L, Scandling J, Sorensen J, Mulloy L, Light J, Corwin C, Danovitch G, Wachs M, VanVeldhuisen P, Salm K, Tolzman D and Fitzsimmons WE. Randomized trial of tacrolimus (Prograf) in combination with azathioprine or mycophenolate mofetil versus cyclosporin (Neoral) with mycophenolate mofetil after cadaveric kidney transplantation. Transplantation 2000;69:834-41.

2. Mayer AD, Dmitrewski J, Squifflet J-P, Besse T, Grabensee B, Klein B, Eigler FW, Heemann U, Pichlmayr R, Behrend M, Vanrenterghem Y, Donck J, Hooff J van, Christiaans M, Morales JM, Andres A, Johnson RWG, Short C, Buchholz B, Rehmert N, Land W, Schleibner S, Forsythe JLR, Talbot D, Neumayer H-H, Hauser I, Ericzon B-G, Brattström C, Claesson K, Mühlbacher F and Pohanka E. Multicenter randomized trial comparing tacrolimus (FK 506) and cyclosporin in the prevention of renal allograft rejection. A report of the European Tacrolimus Multicenter Renal Study Group. Transplantation 1997;64:436-43.

3. Pirsch JD, Miller J, Deierhoi MH, Vincenti F and Filo RS for the FK506 Kidney Transplant Study Group. A comparison of tacrolimus (FK506) and cyclosporine for immunosuppression after cadaveric renal transplantation. Transplantation 1997;63:977-83.

4. Plosker GL and Foster RH. Tacrolimus. A further update of its pharmacology and therapeutic use in the management of organ transplantation. Drugs 2000;59:323-89.

5. Chakrabarti P, Wong HY, Scantlebury VP, Jordan ML, Vivas C, Ellis D, Lombardozzi-Lane S, Hakala TR, Fung JJ, Simmons RL, Starzl TE and Shapiro R. Outcome after steroid withdrawal in pediatric renal transplant patients receiving tacrolimus-based immunosuppression. Transplantation 2000;70:760-4.

6. Hricik DE, Kupin WL and First MR. Steroid-free immunosuppression after renal transplantation. J Am Soc Nephrol 1994;4(S8):S10-6.

7. Oppenheimer F. Steroid withdrawal in renal transplant recipients. Transplant Proc 2000;32 (S1A):14S-5.

8. Schulak JA and Hricik DE. Steroid withdrawal after renal transplantation. Clin Transplant 1994;8:211-6.

9. Shapiro R, Jordan ML, Scantlebury VP, Vivas C, Gritsch HA, Corry RJ, Egidi F, McCauley J, Ellis D, Gilboa N et al. The superiority of tacrolimus in renal transplant recipients - the Pittsburgh experience. Clin Transplant 1995;9:199-205.

10. Boots JMM, Duijnhoven EM, Christiaans MHL, Nieman FHM, Suylen RJ van and Hooff JP van. Single center experience with tacrolimus versus cyclosporin-Neoral in renal transplant recipients. Transpl Int 2001;14:370-83.

11. Moochhala SM, Lee EJD, Earnest L, Wong JYY and Ngoi SS. Inhibition of drug metabolism in rat and human liver microsomes by FK 506 and cyclosporine. Transplant Proc 1991;23: 2786-8.

12. Omar G, Shah IA, Thomson AW, Whiting PH and Burke MD. FK 506 inhibition of cyclosporine metabolism by human liver microsomes. Transplant Proc 1991;23:934-5. 
13. Piekoszewski W, Chow FS and Jusko WJ. Pharmacokinetic and pharmacodynamic effects of coadministration of methylprednisolone and tacrolimus in rabbits. J Pharmacol Exp Ther 1994;269:103-9.

14. Sattler M, Guengerich FP, Yun CH, Christians U and Sewing KF. Cytochrome P-450 3A enzymes are responsible for biotransformation of FK506 and rapamycin in man and rat. Drug Metab Dispos 1992;20:753-61.

15. Shah IA, Whiting PH, Omar G, Thomson AW and Burke MD. Effects of FK 506 on human hepatic microsomal cytochrome P-450-dependent drug metabolism in vitro. Transplant Proc 1991;23:2783-5.

16. Sewing KF. Pharmacokinetics, dosing principles and blood level monitoring. Transplant Proc 1994;26:3267-9.

17. Lo A and Burckart GJ. P-glycoprotein and drug therapy in organ transplantation. J Clin Pharmacol 1999;39:995-1005.

18. Christiaans M, Duijnhoven E van, Beysens T, Undre N, Schäfer A and Hooff J van. Effect of breakfast on the oral bioavailability of tacrolimus and changes in pharmacokinetics at different times posttransplant in renal transplant recipients. Transplant Proc 1998;30:1271-3.

19. Tredger JM, Gilkes CD and Gonde CE. Performance of the Imx tacrolimus II assay and practical limits of detection. Clin Chem 1999;45:1881-2.

20. Wallemacq PE, Leal T, Besse T, Squifflet J-P, Reding R, Otte J-B, Lerut J and Hassoun A. Imx tacrolimus II vs Imx tacrolimus microparticle enzyme immunoassay evaluated in renal and hepatic transplant patients. Clin Chem 1997;43: 1989-91.

21. Boots JMM, Duijnhoven EM van, Christiaans MHL, Wolffenbuttel BHR and Hooff JP van. Glucose metabolism in renal transplant recipients on tacrolimus: the effect of steroid withdrawal and tacrolimus trough level reduction. J Am Soc Nephrol 2002;13:221-7. 


\section{Chapter 5}

\section{Early steroid withdrawal in renal transplantation with tacrolimus dual therapy: A pilot study}

JMM Boots, MHL Christiaans, EM van Duijnhoven, R-J van Suylen, JP van Hooff 


\section{Abstract}

\section{Introduction}

With tacrolimus-based immunosuppression, it appears safe to withdraw steroids three to six months after renal transplantation. We hypothesized whether steroids could also be safely withdrawn early after transplantation.

\section{Methods}

Sixty-two patients $\left(1^{\text {st }}\right.$ or $2^{\text {nd }}$ transplant, with no prior immunological failure, and current PRA $<50 \%$ ), treated with tacrolimus, were prospectively randomized to stop steroids (10 $\mathrm{mg}$ prednisolone) after day seven post-transplantation [stop group (STOP), $n=28]$ or to gradually wean off steroids in three to six months (tapering group (TAP), $\mathrm{n}=34$ ). Analyses were performed on an intention-to-treat basis.

\section{Results}

After a median follow-up of 2.7 years, patient and graft survival were $97 \%$ and $90 \%$ and comparable between both groups ( $P=0.11$ and $P=0.13$, respectively). The incidence of acute rejection was $29 \%$ (STOP) versus 33\% (TAP) $(P=0.30)$. The time to the first rejection was median 35 days (STOP) versus 11 days (TAP) $(P=0.19)$. The severity of the rejections (1997 Banff-classification) was comparable $(P=0.57)$. Creatinine clearance and proteinuria were similar $(P>0.70)$. The incidence of infections was comparable $(P>0.10)$. The incidence of new-onset diabetes mellitus, defined as the use of anti-diabetic medication, was 8.0\% (STOP) versus $30.3 \%$ (TAP) $(P=0.04)$. All cases occurred in the STOP group after one year, while all cases occurred in the TAP group in the first four months $(P<0.001)$.

\section{Conclusions}

Compared to tapering in three to six months, stopping steroids one week posttransplantation results in comparable patient and graft survival and in a similar incidence of acute rejections. The incidence of new-onset diabetes may be reduced. The immunosuppressive benefit of adding $10 \mathrm{mg}$ prednisolone to tacrolimus seems to be limited. 


\section{Introduction}

The role of steroids in maintenance regimens has been questioned because chronic use of steroids gives rise to serious side effects ${ }^{1}$. Recently, in a metaanalysis, Kasiske et al. ${ }^{2}$ showed that in patients on cyclosporin, in whom steroids were withdrawn, acute rejection occurred significantly more often and that the relative risk of graft failure was also higher. However, in the 1990s, tacrolimus was introduced as a more potent immunosuppressive drug than cyclosporin ${ }^{3,4}$. The Pittsburgh group demonstrated that steroids could be safely withdrawn in a majority of the patients after the first six months post-transplantation ${ }^{5,6}$. After withdrawal, the incidence of acute rejection was $5 \%{ }^{6}$. We were able to withdraw steroids in nearly $80 \%$ of the tacrolimus-treated patients, without the occurrence of acute rejections. Seventy percent of our patients could be successfully treated with tacrolimus monotherapy ${ }^{7}$.

Currently, with the development of new immunosuppressants, transplantation with early steroid withdrawal ${ }^{8,9}$ or transplantation even without steroids ${ }^{10}$ regained interest. However, all these regimens consist of at least three immunosuppressive drugs and this approach was restricted to recipients with a low risk of acute rejection. This may result in over-immunosuppression for an important part of these patients. In our opinion, tacrolimus dual therapy is sufficient to achieve a reasonable acute rejection rate in these patients ${ }^{11}$. In addition, we believe that the immunosuppressive capacity of tacrolimus is that strong that the additional benefit of steroids can be doubted and that they can be safely withdrawn early after transplantation. In this prospectively randomized single centre pilot study, we investigated the safety of stopping steroids with tacrolimus dual therapy one week post-transplantation compared to our standard regimen of gradually tapering steroids in three to six months. In contrary to previous studies with early steroid withdrawal, the protocol was not restricted to low risk recipients, but patients with a moderate risk were also included.

\section{Patients and methods}

From November 1997 until July 2000, patients were prospectively enrolled in the study. To avoid selection bias, no patients were included from October 1998 until October 1999, because a multi-centre trial was performed during that period. Patients were eligible to participate, if they had a low to moderate immunological profile for rejection, defined as: (1) first transplant or second transplant, in which graft failure was not due to immunological causes, and (2) less than $50 \%$ panel reactive class-I HLA-antibodies (PRA) in the current serum. 
Patients were excluded if they: (1) were younger than 18 years, (2) had an HLAidentical living donor, (3) had no match on either the HLA-B or -DR locus, (4) had a previously known allergy to tacrolimus or macrolide antibiotics, and (5) refused to give informed consent.

At the time of transplantation, randomisation was performed by opening a closed opaque numbered envelope. Patients were randomized in a 1:1 ratio either to stop steroids after day 7 post-transplantation or after month 3 .

Follow-up data were collected until May 1, 2001, the date of death or graft failure.

\section{Immunosuppressive protocol}

All patients received a routine blood transfusion prior to transplantation unless they had been transfused previously.

Tacrolimus was administered orally, $0.10-0.15 \mathrm{mg} / \mathrm{kg}$ body weight b.i.d., and started within 12 hours prior to transplantation. Dosages were adjusted on 12 hour trough levels (IMx, Abbot Laboratories, Abbott Park, IL, USA). The target levels were $15-20 \mathrm{ng} / \mathrm{ml}$ in the first two weeks, $10-15 \mathrm{ng} / \mathrm{ml}$ in the following two weeks, and thereafter gradually reduced until $5-7 \mathrm{ng} / \mathrm{ml}$ after six months.

All patients received a steroid bolus of 125 methylprednisolone prior to surgery. From day 1 onwards, patients received $10 \mathrm{mg}$ prednisolone orally.

In the stop group (STOP), steroids were stopped at day 8 or, if the tacrolimus trough level did not meet the target criteria, as soon as the tacrolimus trough level was stable above $15 \mathrm{ng} / \mathrm{ml}$.

In the tapering group (TAP), steroids were reduced after one month to $7.5 \mathrm{mg}$ and one month later to $5 \mathrm{mg}$. Thereafter, the steroids were withdrawn within one to three months. If there were side effects, e.g. severe or recurrent infections or the development of diabetes mellitus, steroids were reduced or stopped earlier.

The cumulative prednisolone dose was calculated in both groups, by considering the prednisolone equivalence of methylprednisolone as 1.25 and of hydrocortisone as 0.25 .

Rejections were treated with a course of three bolus of 500-1000 mg methylprednisolone on alternate days. In cases of steroid resistance, a 10-day course of rabbit anti-thymocyte globulin (r-ATG, RIVM, Bilthoven, the Netherlands) was used. When rejections occurred after steroid withdrawal, steroids were restarted. Generally, mycophenolate mofetil (MMF) was added unless the rejection was mild (small rise in serum creatinine and a biopsy score of Banff-grade $1 \mathrm{~A}$ or borderline) or contra-indications were present (infections).

\section{Endpoints}

Primary endpoints were: (1) patient survival, (2) graft survival, and (3) the incidence of a first acute rejection episode in the first six months posttransplantation. Secondary endpoints were: (4) creatinine clearance, (5) 
proteinuria, (6) the incidence of new-onset diabetes mellitus, and (7) the incidence of infections.

Graft loss was defined as return to dialysis, independent of its cause, or death with a functioning graft. Acute rejection was defined as any rejection treatment in the first six months. There was clinical suspicion of rejection if there was an unexplained rise or insufficient decrease in serum creatinine, with or without other signs, such as fever, tenderness of the graft, decreased renal perfusion on nuclear scan, or hypertension. Unless there was a contra-indication for biopsy, rejection episodes were confirmed with an ultrasound-guided needle core biopsy. In the case of delayed graft function (need for dialysis in the first week after transplantation), a protocol biopsy was performed at week 1 to rule out rejection. A pathologist classified all biopsies in a blinded fashion according to the 1997Banff classification ${ }^{12}$.

Creatinine clearance was calculated using the Cockcroft Gault formula. Proteinuria was expressed as protein/creatinine ratio $(\mathrm{mg} / \mathrm{mg})^{13}$ and assessed in a 24-hour urine sample. A value of 0.2 or less is considered normal. The protein/creatinine ratio correlates well with the actual 24-hour protein excretion ${ }^{13}$. Patients were classified as having new-onset diabetes mellitus if oral anti-diabetic drugs or insulin were used at any time after transplantation, except for an incidentally raised glucose level during stress or methylprednisolone bolus, and if no diagnosis of diabetes had been established before transplantation.

Except for prophylactic use, any treatment episode with anti-microbial drugs was considered to be an infection. No Pneumocystis carinii or Cytomegalovirus (CMV) prophylaxis was prescribed. CMV was treated if CMV pp65-expression on leucocytes by antigenemia testing was positive or if a positive culture was obtained, concomitant with clinical signs or during anti-rejection treatment.

\section{Statistical analysis}

Statistical analyses were performed with the SPSS software package for Windows, version 10.0 (SPSS, Inc, Chicago, IL.). For comparison of baseline characteristics, we used Student's t-test for independent samples or, in the case of a nonparametrical distribution, the Mann-Whitney test. For relationship between categorical variables, Pearson's Chi-Square-test was used.

Patient and graft survival, and the incidence of acute rejection were established using a Kaplan-Meier survival analysis. Differences between the groups were estimated with the $\log$ rank test. The contribution of supposed risk factors for both groups was determined by Cox regression analysis, by both stepwise forward selection and backward elimination of risk factors. Differences between the steroid withdrawal group (STOP vs. TAP) were always tested for in analysis. A basic model was tested for, which did include only direct odds ratio effects that were statistically significant. When two factors were highly associated, the one that was more strongly related to the outcome variables was chosen. The possibility of 
interactions between factors in the basic model (different relative effects within different subgroups) was not taken into account, since too many additional terms would have had to be included to test for such effects. The following supposedly relevant risk factors were included in the analysis: recipient age (years), recipient gender (male vs. female), race (Caucasian vs. non-Caucasian), HLA-DR mismatch ( 0 vs. $\geq 1$ ), current PRA-level ( $<5 \%$ vs. $\geq 5 \%$ ), transplant-number ( 1 vs. 2 ), the type of transplant (heart-beating vs. non heart-beating vs. living), donor age (years), donor gender (male vs. female), and the presence of a first acute rejection (yes vs. no). The presence of an acute rejection was not included in the Cox regression analysis of acute rejection.

Secondary endpoints were analyzed with the same instruments as used for the baseline characteristics. Creatinine clearance and proteinuria were compared at the following time points: 3, 6, 9, and 12 months post-transplantation. Differences between month three and year one were compared with the paired samples t-test, the Wilcoxon signed ranks test, in the case of a non-parametrical distribution, or the McNemar test for categorical variables.

Analyses were made on an intention-to-treat basis. For the analysis of new-onset diabetes mellitus, the patients with pre-existing diabetes were excluded.

In the past, we achieved a $60 \%$ acute rejection rate with Neoral and a $23 \%$ acute rejection rate with tacrolimus and steroids 7 . To detect an increase in the acute rejection rate from $25 \%$ to $60 \%, 60$ patients would be needed to have a power of $80 \%$ with an alpha of 0.05 .

$P$-values less than 0.05 were considered as statistically significant.

\section{Results}

During the study-period, 135 consecutive kidney transplantations were performed (Figure 5.1). From October 1998 until September 1999, 59 consecutive patients participated in a multi-center trial. Of the remaining 76 patients, 14 did not meet the selection criteria: HLA-identical sibling $(n=1)$, high-risk candidates $(n=7)$, and refusal of informed consent $(\mathrm{n}=6)$. Thus, 62 patients were enrolled in the present study. Twenty-eight patients were randomized to the STOP group and 34 to the TAP group. The median follow-up time was 2.7 years (range: $0.9-3.4$ years).

The baseline characteristics of the patients are shown in Table 5.1. The groups were comparable, except for the fact that the STOP group consisted of more women $\left(P=0.05, \chi^{2}\right.$-test $)$ and had a better HLA-DR match $\left(P=0.03, \chi^{2}\right.$-test). 


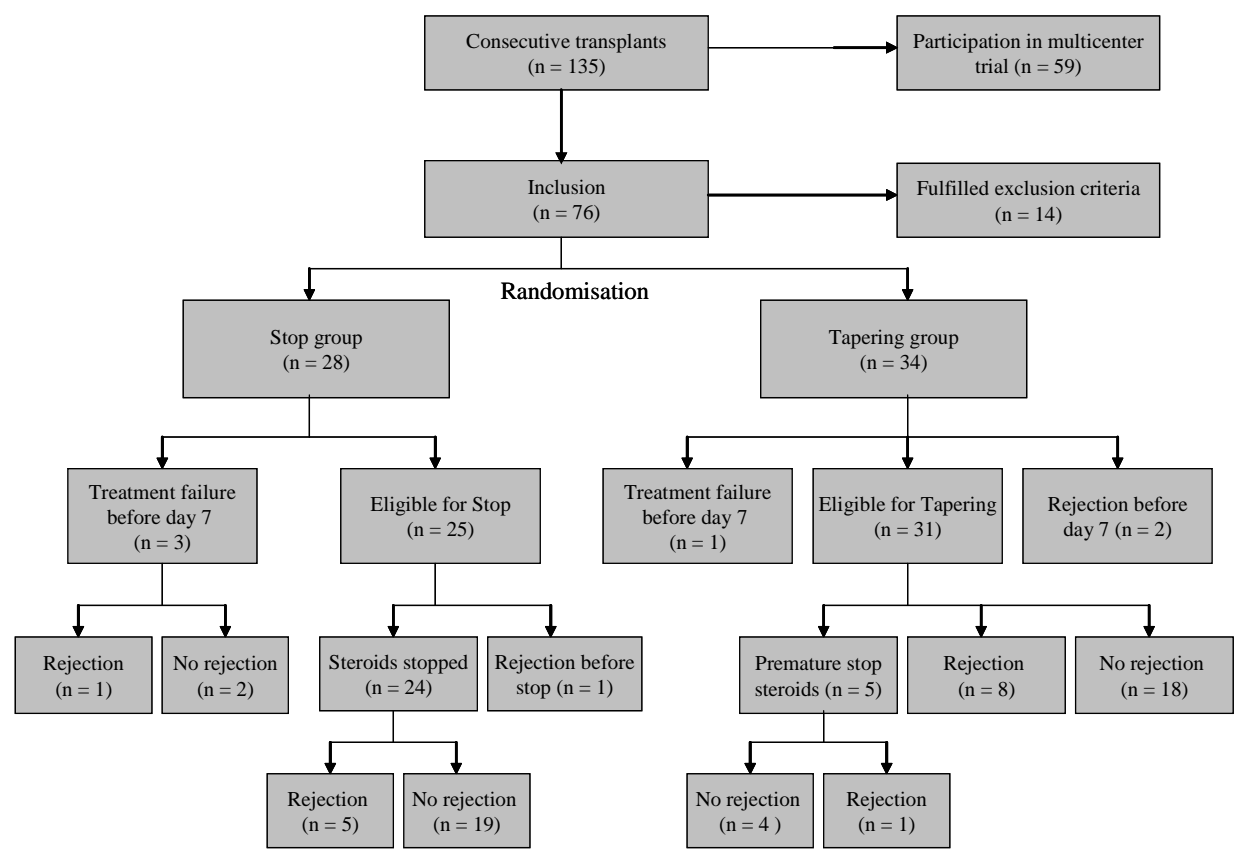

Figure 5.1 Flow-chart of the protocol and the course of the patients

One patient in the Stop group experienced an acute rejection one day after conversion to cyclosporin at day 4 because of tacrolimus allergy.

\section{Patients}

A flow chart of the enrolled patients is presented in Figure 5.1. In the STOP group, three patients had treatment failure before day seven: one died due to myocardial infarction, one experienced thrombosis, and one underwent conversion to cyclosporin because of tacrolimus allergy. After conversion, the latter patient was also treated for acute rejection and for hemolytic uremic syndrome (HUS). An additional patient in the STOP group died of a myocardial infarction on day 17.

In the TAP group, one patient had treatment failure before day seven (thrombosis) and two patients were converted to other regimens because of HUS (day 8) and presumed drug-induced pneumonia (day 133), respectively.

\section{Immunosuppressive protocol}

In the STOP group, prednisolone was stopped after a median of 10 days (range 7-18 days) in 24 patients. According to the protocol, steroid withdrawal was 
postponed in 12 patients for a median of eight days (range 4-11 days), because of a tacrolimus trough level below $15 \mathrm{ng} / \mathrm{ml}$. In one of these patients, prednisolone was continued because of a rejection before planned steroid withdrawal.

Table 5.1 Baseline characteristics

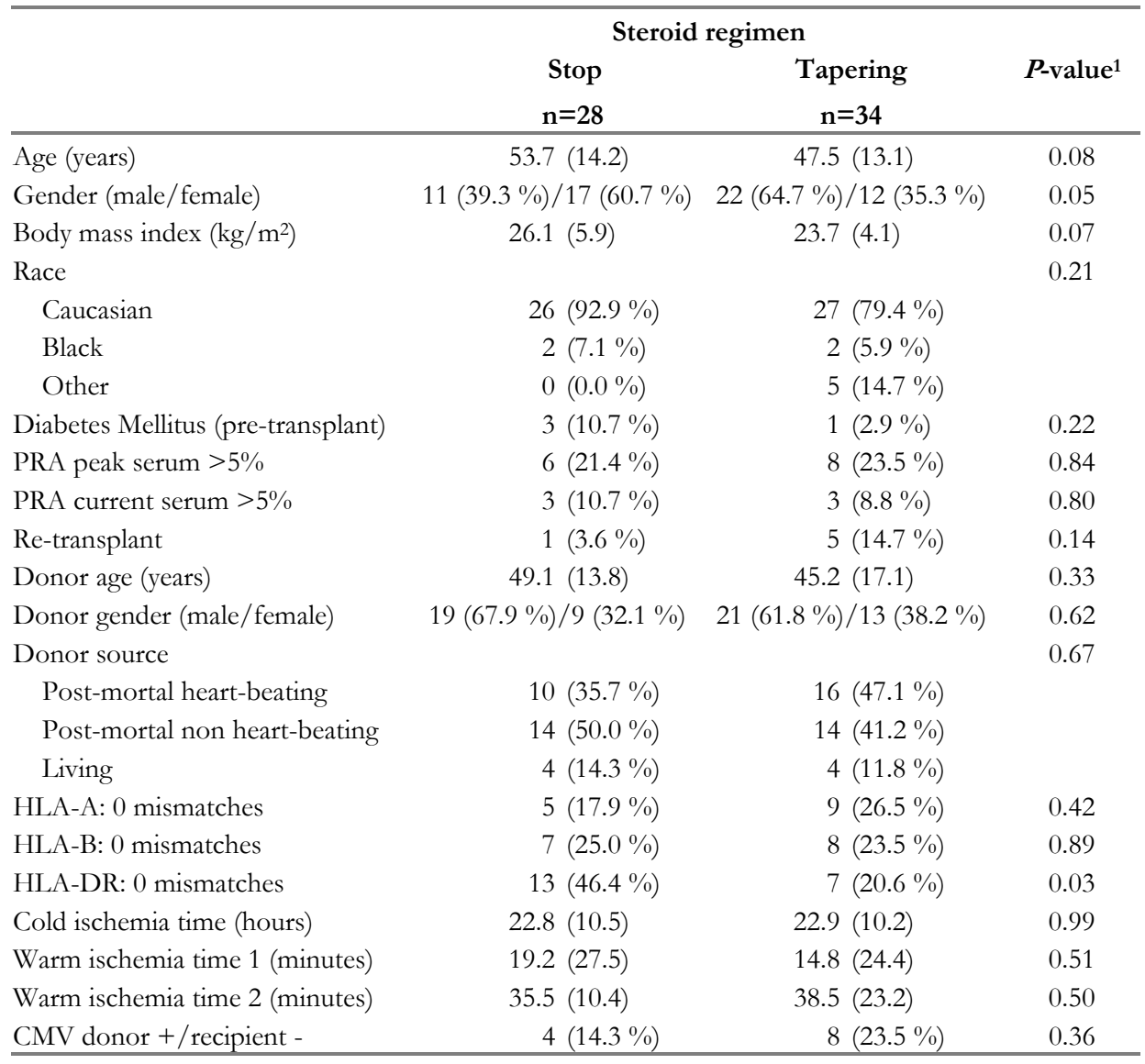

The data are expressed as mean (SD) or numbers (\%). ${ }^{1}=$ Student's t-test or Pearson's $\chi 2$-test (categorical variables)

In the TAP group, prednisolone was tapered according to the protocol to $5 \mathrm{mg}$ in five patients due to rejection. In 27 patients, prednisolone was stopped after a median of 98 days (range 17-207 days). In eight patients prednisolone was stopped before month three due to severe infection $(n=5)$ or diabetes $(n=3)$ after a median of 40 days (range 17-64 days). 
The median cumulative prednisolone equivalence dose in the first six months post-transplantation was $403 \mathrm{mg}$ (range 166-8904 mg) in the STOP group versus $1256 \mathrm{mg}(186-6390 \mathrm{mg})$ in the TAP group ( $P=0.002$, Mann-Whitney test).

In the STOP group, tacrolimus trough levels were significantly higher after steroid withdrawal at week 2 and 3 ( $P=0.02$ and 0.01, respectively, t-test; Figure 5.2). From week six until week 11, tacrolimus trough levels were slightly higher in the TAP group ( $P=0.03-0.12$, t-test). In rejectors, tacrolimus trough levels were similar in both groups at the time of the first rejection: $13.2 \mathrm{ng} / \mathrm{ml}$ in the STOP group versus $13.7 \mathrm{ng} / \mathrm{ml}$ in the TAP group ( $P=0.83$, t-test).

At the end of the follow-up, $73.9 \%$ of the patients in the STOP group versus $78.1 \%$ in the TAP group used tacrolimus monotherapy $\left(P=0.72, \chi^{2}\right.$-test $)$.

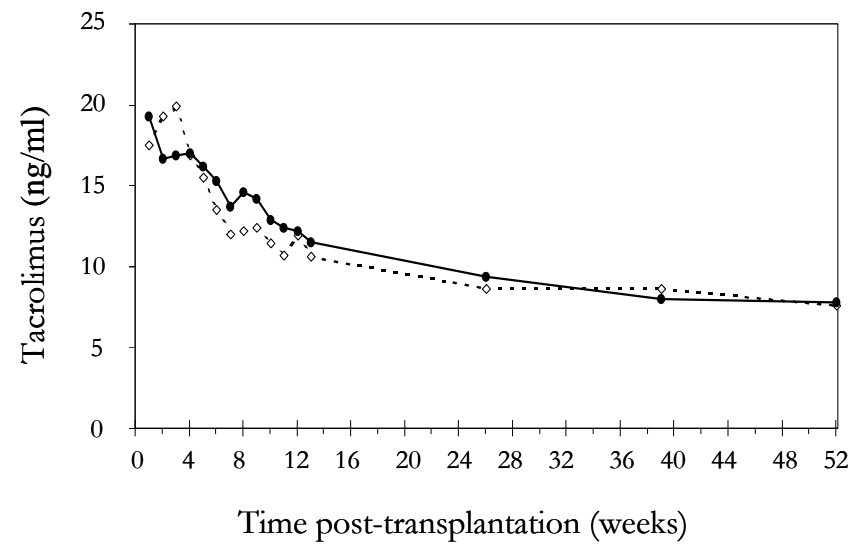

Figure 5.2 Tacrolimus trough levels

Stop group: dotted line. Trough levels were significantly higher in week 2 and 3 in the Stop group ( $P=0.02$ and $P=0.01$, t-test, respectively). In the Tapering group, trough levels were higher from week 6 until week $11(P=0.03-P=0.12$, t-test).

\section{Patient and graft survival}

The patient survival after a median of 2.7 years was $97 \%$ and comparable between the two groups ( $P=0.11, \log$ rank test). Because of the low number of events, no Cox regression analysis of the potential risk factors could be performed.

The graft survival after a median of 2.7 years was $90 \%$ and also comparable between the groups $(P=0.13, \log$ rank test). None of the risk factors included in the Cox regression analysis contributed significantly. Three grafts were lost due to thrombosis $(3,4$, and 17 days post-transplantation), two of which were derived from non-heart-beating donors. Two were included in the STOP group and one 
in the TAP group (day three). One graft in the STOP group was lost due to ongoing rejection and one graft in the TAP group was lost due to chronic pyelonephritis with Candida Glabrata.

\section{Acute rejection}

The incidence of acute rejection was $29 \%$ in the STOP group versus $33 \%$ in the TAP group ( $P=0.30, \log$ rank test; Figure 5.3$)$. By Cox regression analysis none of the potential risk factors contributed significantly to the results $(P>0.15)$. Up until day 7 , three rejections occurred: one $(1.6 \%)$ in the STOP group versus two $(3.2 \%)$ in the TAP group $(P=0.67$, Chi-Square test). The median time to the first rejection was 35 days (range 5-84 days) in the STOP group versus 11 days (range 6-48 days) in the TAP group ( $P=0.19$, Mann-Whitney test). In the STOP group, the rejection occurred at a median of 38 days (range 10-75 days) after steroid withdrawal.

Within the first six months, 49 biopsies were performed in 29 patients. 13 patients $(46.4 \%)$ in the STOP group versus $16(47.1 \%)$ in the TAP group. The indications for the first biopsy were: delayed graft function $(n=7)$, creatinine rise $(n=20)$, insufficient creatinine decrease $(n=1)$, or proteinuria $(n=1)$. The indications were comparable between the groups $\left(P=0.45, \chi^{2}\right.$-test). The Banff score of the rejections was comparable, both for the score in the first biopsy as for the highest score in all biopsies of a patient $(P=0.57$ and $P=0.79$, Chi-Square test, respectively; Table 5.2).

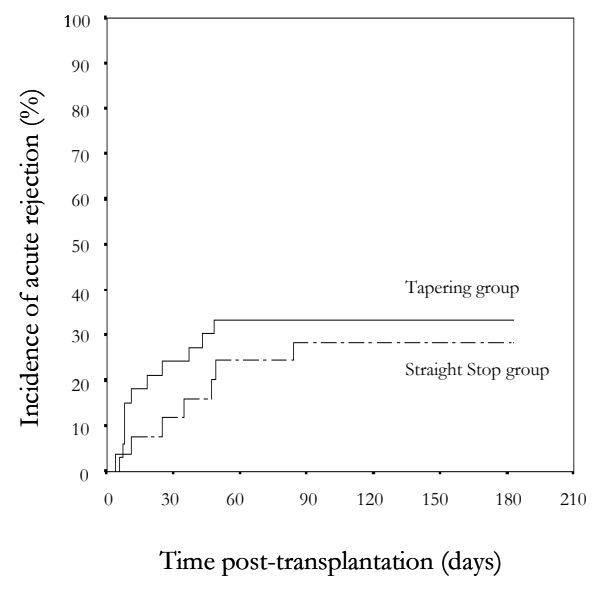

Figure 5.3 The incidence of acute rejections

Kaplan-Meier curve of the incidence of acute rejections in the first six months posttransplantation. The incidence was $29 \%$ in the Stop group (dotted line) versus $33 \%$ in the Tapering group $(P=0.30, \log$ rank test $)$. 
Table 5.2 The blinded histology scores of the biopsies in patients treated for acute rejection (1997 Banff-classification).

\begin{tabular}{llcc}
\hline & $\begin{array}{c}\text { Stop } \\
(\mathbf{n}=7)\end{array}$ & $\begin{array}{c}\text { Steroid } \\
\text { Tapering } \\
(\mathbf{n}=\mathbf{9}) \mathbf{b}\end{array}$ & $\begin{array}{c}\boldsymbol{P} \text {-value } \\
(\boldsymbol{\chi} \text { 2-test) }\end{array}$ \\
\hline $\begin{array}{l}\text { Banff score first rejection } \\
\text { borderline }\end{array}$ & $3(43 \%)$ & $2(22 \%)$ & 0.57 \\
1 A & $3(43 \%)$ & $5(56 \%)$ & \\
1 B & $0(0 \%)$ & $0(0 \%)$ & \\
2 A & $1(14 \%)$ & $1(11 \%)$ & \\
2 B & $0(0 \%)$ & $1(11 \%)$ & \\
Highest Banff score & & $(\mathrm{n}=10) \mathrm{c}$ & \\
borderline & $3(43 \%)$ & $2(20 \%)$ & \\
1 A & $2(29 \%)$ & $4(40 \%)$ & \\
1 B & $1(14 \%)$ & $2(20 \%)$ & \\
2 A & $1(14 \%)$ & $1(10 \%)$ & \\
2 B & $0(0 \%)$ & $110 \%)$ & \\
\hline
\end{tabular}

$\mathrm{a}=$ of any biopsy performed within one patient. $\mathrm{b}=$ two patients were treated for rejection on clinical criteria and had no biopsy performed because of clotting disorders. ${ }^{c}=$ one of these patients was treated for a recurrent rejection and had then a biopsy performed after correction of the coagulant status

All rejections but one were steroid-sensitive. One patient in the STOP group had biopsy-proven steroid-resistant rejection and was successfully treated with r-ATG. Recurrent rejections occurred in $7.1 \%$ of the STOP group versus $8.8 \%$ of the TAP group ( $P=0.81$, Chi-Square test). One graft was lost due to recurrent rejections in the STOP group. However, because of an early rejection before scheduled steroid withdrawal, the steroids were never stopped according to the protocol. Due to CMV-disease and pre-existing extensive sclerotic lesions renewed anti-rejection treatment had been contra-indicated.

Late acute rejection occurred in one patient in the STOP group (day 427) versus two in the TAP group (day 423 and 523, respectively). In the TAP group, the late rejections were due to proven bad compliance. All late acute rejections were steroid-sensitive.

\section{Creatinine clearance and proteinuria}

There were no differences in creatinine clearance and proteinuria at the different time points ( $\mathrm{P}>0.70$, t-test and Mann-Whitney test, respectively; Table 5.3). Both creatinine clearance and proteinuria improved from month three to year one posttransplantation $(P<0.001$, paired samples t-test and $P=0.02$, Wilcoxon signed ranks test, respectively). One patient in the STOP group had recurrence of focal 
segmental glomerulosclerosis. In the STOP group, one patient had proteinuria in the normal range at month three, this deteriorated to significant proteinuria at year one. This occurred in two patients in the TAP group ( $P=0.81$, Chi-Square test).

Blood pressure and the use of anti-hypertensive drugs were comparable at all time points $(P>0.30$, t-test and $P>0.10$, Mann-Whitney test, respectively; Table 5.3). From month three to year one, both blood pressure and the use of antihypertensive drugs remained unchanged $(P=0.37$, paired samples t-test and $P=0.80$, Wilcoxon signed ranks test, respectively). Also, the use of ACE inhibitors was comparable $(P=0.29$, Chi-Square test $)$ and did not change in time $(P=0.25$, McNemar test).

\section{New-onset diabetes mellitus}

The incidence of new-onset diabetes mellitus was significantly lower in the STOP group than in the TAP group: $8.0 \%$ versus $30.3 \%(P=0.04$, Chi-Square test; Table 5.3). Seven patients in the TAP group $(20.6 \%)$ had to be treated with insulin versus 0 patients in the STOP group ( $P=0.01$, Chi-Square test). All patients in the TAP group developed diabetes in the first four months post-transplantation, while in the STOP group, this occurred only after one year. The median time to the development of diabetes was 631 days (range 547-714 days) in the STOP group versus 31 days (7-113 days) in the TAP group ( $P<0.001$, Mann-Whitney test).

\section{Infection}

The incidences of fungal, viral and bacterial infections were comparable between the two groups $(P>0.10$, Chi-Square test; Table 5.3). In the TAP group one transplant was lost at day 307 due to chronic pyelonephritis with Candida Glabrata. 
Table 5.3 Secondary outcome.

\begin{tabular}{|c|c|c|c|c|c|}
\hline & \multicolumn{4}{|c|}{ Steroid regimen } & \multirow[b]{2}{*}{$P$-value } \\
\hline & n & Stop & $\mathbf{n}$ & Tapering & \\
\hline \multicolumn{6}{|l|}{ Creatinine clearance (ml/min) } \\
\hline month 3 & 23 & $43.0(18.8)$ & 33 & $44.6(24.0)$ & $0.78^{\mathrm{a}}$ \\
\hline month 6 & 23 & $50.2(20.5)$ & 33 & $48.4(20.1)$ & $0.75^{\mathrm{a}}$ \\
\hline month 9 & 23 & $52.8(20.9)$ & 32 & $53.5(21.6)$ & $0.91^{\mathrm{a}}$ \\
\hline year 1 & 18 & $55.3(21.6)$ & 28 & $53.2(17.9)$ & $0.73^{a}$ \\
\hline \multicolumn{6}{|l|}{ Protein/creatinine ratio $(\mathrm{mg} / \mathrm{mg})$} \\
\hline month 3 & 23 & $0.24(0.04-18.34)$ & 33 & $0.25(0.07-1.53)$ & $0.76^{\mathrm{b}}$ \\
\hline month 6 & 23 & $0.17(0.03-18.32)$ & 32 & $0.23(0.05-3.12)$ & $0.97^{\mathrm{b}}$ \\
\hline month 9 & 23 & $0.18(0.09-16.46)$ & 31 & $0.18(0.05-3.48)$ & $0.79 \mathrm{~b}$ \\
\hline year 1 & 18 & $0.17(0.02-18.71)$ & 28 & $0.18(0.06-3.03)$ & $0.91^{\mathrm{b}}$ \\
\hline Normal range at year 1 & & $11(61.1 \%)$ & & $14(51.9 \%)$ & $0.33^{c}$ \\
\hline Proteinuria at year 1 & & $6(33.3 \%)$ & & $13(48.1 \%)$ & \\
\hline Nephrotic range at year 1 & & $1(5.6 \%)$ & & $0(0.0 \%)$ & \\
\hline \multicolumn{6}{|l|}{ Mean blood pressure (mmHg) } \\
\hline month 3 & 23 & $99.7(13.0)$ & 33 & $96.6(10.4)$ & $0.32^{\mathrm{a}}$ \\
\hline month 6 & 23 & $96.0(12.1)$ & 32 & $94.2(9.7)$ & $0.55^{\mathrm{a}}$ \\
\hline month 9 & 23 & $95.4(12.5)$ & 31 & $96.3(10.5)$ & $0.79^{a}$ \\
\hline year 1 & 18 & $98.1(10.7)$ & 28 & $95.1(9.4)$ & $0.33^{a}$ \\
\hline \multicolumn{6}{|l|}{ Number of anti-hypertensive drugs } \\
\hline month 3 & 23 & $2(0-5)$ & 33 & $1(0-4)$ & $0.67 \mathrm{~b}$ \\
\hline month 6 & 23 & $2(0-5)$ & 32 & $1(0-4)$ & $0.13^{b}$ \\
\hline month 9 & 23 & $2(0-5)$ & 31 & $1(0-4)$ & $0.20^{\mathrm{b}}$ \\
\hline year 1 & 18 & $2(0-5)$ & 28 & $1(0-3)$ & $0.20^{\mathrm{b}}$ \\
\hline New-onset diabetes mellitus & 25 & & 33 & & \\
\hline total & & $2(8.0 \%)$ & & $10(30.3 \%)$ & $0.04^{c}$ \\
\hline in first year & & $0(0.0 \%)$ & & $10(30.3 \%)$ & $0.002^{\mathrm{c}}$ \\
\hline time after transplantation (days) & & $631(547-714)$ & & $31(7-113)$ & $<0.001^{b}$ \\
\hline use of insulin & & $0(0.0 \%)$ & & $7(21.2 \%)$ & $0.01^{\mathrm{c}}$ \\
\hline Infections & 28 & & 34 & & \\
\hline none & & $10(35.7 \%)$ & & $11(32.4 \%)$ & $0.78^{\mathrm{c}}$ \\
\hline urinary tract & & $18(64.3 \%)$ & & $15(44.1 \%)$ & $0.11^{\mathrm{c}}$ \\
\hline $\mathrm{CMV}$ & & $2(7.7 \%)$ & & $6(17.6 \%)$ & $0.22^{\mathrm{c}}$ \\
\hline other & & $8(28.6 \%)$ & & $9(26.5 \%)$ & $0.85^{c}$ \\
\hline
\end{tabular}

${ }^{a}=$ mean (SD); t-test; ${ }^{b}=$ median (range); Mann-Whitney test; ${ }^{c}=$ numbers $(\%) ; \chi^{2}$-test

\section{Discussion}

In this study, we compared the safety and efficacy of steroid cessation one to two weeks post-transplantation with tapering of the steroids in three to six months in patients on dual therapy. We excluded extremely low-risk recipients $(n=1)$ and 
high-risk recipients $(\mathrm{n}=7)$. Thus, this minimal immunosuppressive approach was applied to almost $90 \%$ of our transplant population (62 out of 70 potential renal transplant candidates).

After a median of 2.7 years follow-up, overall patient survival was $97 \%$, which is similar to other studies of tacrolimus-based immunosuppression, 3 ,4,6,14-17. Two patients in our study died of a myocardial infarction. Before transplantation, one patient had already extensive cardiovascular disease and the other patient had type 1 diabetes mellitus.

Overall graft survival, including death with a functioning graft, was $90 \%$. Our population had a moderate immunological risk of acute rejection. Forty-five percent of the grafts were derived from non-heart-beating donors and the donor age was higher than in two other studies with tacrolimus and steroids 6,14 . Notwithstanding our extended donor criteria, graft survival was comparable to that found in other studies using tacrolimus and steroids ${ }^{6,14-16}$ and the main trials ${ }^{3,4}$.

The incidence of thrombosis (4.8\%) is high compared to the reported incidence of $0.5-2 \%{ }^{3,4}$. This was probably due to the use of marginal donors. Microscopy showed no signs of rejection.

One graft was lost due to recurrent rejection. Because of active CMV-disease and extensive pre-existing sclerotic lesions, it was decided to perform a transplantectomy and to refrain from anti-rejection treatment in this patient.

The overall incidence of acute rejection was $31 \%$. This is comparable to other studies using tacrolimus dual therapy in Europe reporting acute rejection rates between $27 \%$ and $36 \% \%^{15-17}$. Studies from the United States report rates ranging from $44 \%$ to $54 \%{ }^{6,14}$. Some studies also excluded high-risk recipients ${ }^{14,15}$. Besides, in our study, the steroid dose was lower and steroids were withdrawn early. Also, the severity of the acute rejections, according to the Banff-criteria, was comparable to other studies using tacrolimus ${ }^{3,4,14}$.

Five patients experienced an acute rejection after cessation of steroids. However, the rejections in the tapering group occurred even earlier. Thus, it seems unlikely that the rejections would have been prevented if the steroids were continued.

HLA-DR mismatch, female gender, younger recipient age, PRA-level, retransplantation, and black race are known risk factors for acute rejection. The distribution of some of these factors was not equal between the two groups after randomisation. To test the possibility that these factors influenced the estimation of the non-significance of the type of group, a Cox regression analysis was performed in which the basic model included the above-mentioned risk factors. Neither early steroid withdrawal $(P=0.9)$ nor the known risk factors $(P>0.15)$ contributed to rejection. 
The incidence of recurrent rejections $(8 \%)$ was low and only one steroid-resistant rejection was observed. Moreover, the rejections had no clear negative impact on graft survival, renal function, and proteinuria. Thus, early steroid withdrawal in the vast majority of the transplant population seems to be a safe procedure. Our study also showed no difference between stopping steroids after one week and gradually weaning it in three to six months. Thus, the immunosuppressive benefit of adding $10 \mathrm{mg}$ prednisolone seems to be limited.

With addition of MMF the risk of acute rejection can be reduced to $15 \%{ }^{16}$, but a considerable proportion of the transplant patients will become overimmunosuppressed. Acute rejection is a risk factor for chronic rejection ${ }^{18}$, but the increased risk is mainly the result of vascular rejection ${ }^{19}$ and the risk is low after complete resolution of the acute rejection ${ }^{20}$. The incidence of steroid resistant and vascular rejections in our study was low.

The starting steroid dose from day 1 post-transplantation was $10 \mathrm{mg}$ prednisolone. This is relatively low compared to that used in other studies ${ }^{6,14-17}$. So far, the amount of steroids for adequate immunosuppression has never been clearly defined. It remains unclear whether higher dosing of steroids in tacrolimusbased immunosuppression leads to a lower incidence of acute rejection. However, higher dosages result in more side effects. It has been proven that withdrawal of steroids improves blood pressure ${ }^{21,22}$, lipid profile ${ }^{21,22}$, and insulin resistance ${ }^{23}$, and prevents bone $\operatorname{loss}^{22}$.

In contrast to what might have been expected, the time to the first rejection was longer in the STOP group than in the TAP group, probably due to the significantly higher trough levels after steroid withdrawal in week 2 and 3 . Tacrolimus trough levels are important in preventing acute rejections ${ }^{24}$ and we have proven that stopping steroids increases tacrolimus trough levels in a dosedependant way in $50-60 \%$ of the recipients ${ }^{25,26}$.

Studies with prednisolone withdrawal in cyclosporin-treated patients have demonstrated reduced graft function ${ }^{27}$ and an increased risk of graft failure ${ }^{2}$. No prospective data are available for tacrolimus-treated patients. Renal function ${ }^{28,29}$ and proteinuria ${ }^{30,31}$ one year post-transplantation are important prognostic factors for long-term graft survival.

One year post-transplantation, the mean creatinine clearance was $54 \mathrm{ml} / \mathrm{min}$. Despite our extended donor criteria, this figure is similar to that in the study by Shapiro et al. ${ }^{6}$ and to our previous findings ${ }^{7}$.

Proteinuria improved significantly from a median of $0.24 \mathrm{mg} / \mathrm{mg}$ at month 3 to a median of $0.18 \mathrm{mg} / \mathrm{mg}$ at year 1 post-transplantation, while blood pressure and 
the use of anti-hypertensive drugs, including ACE inhibitors, remained stable. The rate of proteinuria is comparable to what has been reported ${ }^{30,31}$.

Thus, creatinine clearance and proteinuria do not indicate an increased risk of chronic rejection; however, a long-term follow-up is needed to confirm this.

An interesting advantage of early steroid cessation might be a reduction in the incidence of new-onset diabetes mellitus. Also, Grewal et al. ${ }^{9}$ and Cole et al. ${ }^{10}$ reported a low incidence of new-onset diabetes in their studies. However, larger studies are required to estimate the real risk.

In summary, this study showed that, at intermediate-term, it is safe to withdraw low-dose steroids early after renal transplantation in the majority of transplant recipients. Surrogate parameters of long-term graft survival, as creatinine clearance and proteinuria, are comparable. Besides, the incidence of new-onset diabetes mellitus may be reduced. The immunosuppressive benefit of adding low-dose steroids to adequately dosed tacrolimus in these recipients seems to be limited or even absent. 


\section{References}

1. Veenstra DL, Best JH, Hornberger J, Sullivan SD and Hricik DE. Incidence and long-term cost of steroid-related side effects after renal transplantation. Am J Kidney Dis 1999;33:829-39.

2. Kasiske BL, Chakkera HA, Louis TA and Ma JZ. A meta-analysis of immunosuppression withdrawal trials in renal transplantation. J Am Soc Nephrol 2000;11:1910-7.

3. Pirsch JD, Miller J, Deierhoi MH, Vincenti F and Filo RS for the FK506 Kidney Transplant Study Group. A comparison of tacrolimus (FK506) and cyclosporine for immunosuppression after cadaveric renal transplantation. Transplantation 1997;63:977-83.

4. Mayer AD, Dmitrewski J, Squifflet J-P, Besse T, Grabensee B, Klein B, Eigler FW, Heemann U, Pichlmayr R, Behrend M, Vanrenterghem Y, Donck J, Hooff J van, Christiaans M, Morales JM, Andres A, Johnson RWG, Short C, Buchholz B, Rehmert N, Land W, Schleibner S, Forsythe JLR, Talbot D, Neumayer H-H, Hauser I, Ericzon B-G, Brattström C, Claesson K, Mühlbacher F and Pohanka E. Multicenter randomized trial comparing tacrolimus (FK 506) and cyclosporin in the prevention of renal allograft rejection. A report of the European Tacrolimus Multicenter Renal Study Group. Transplantation 1997;64:436-43.

5. Shapiro R, Jordan M, Scantlebury V, Fung J, Jensen C, Tzakis A, McCauley J, Carroll P, Ricordi C, Demetris AJ, Mitchell S, Jain A, Iwaki Y, Kobayashi M, Reyes J, Todo S, Hakala TR, Simmons RL and Starzl TE. FK 506 in clinical kidney transplantation. Transplant Proc 1991;23:3065-7.

6. Shapiro R, Jordan ML, Scantlebury VP, Vivas C, Fung JJ, McCauley J, Randhawa P, Demetris AJ, Irish W, Mitchell S, Hakala TR, Simmons RL and Starzl TE. A prospective randomized trial of FK506-based immunosuppression after renal transplantation. Transplantation 1995;59:485-90.

7. Boots JMM, Duijnhoven EM, Christiaans MHL, Nieman FHM, Suylen RJ van and Hooff JP van. Single center experience with tacrolimus versus cyclosporin-Neoral in renal transplant recipients. Transpl Int 2001;14:370-83.

8. Matas AJ, Ramcharan T, Paraskevas S, Gillingham KJ, Dunn DL, Gruessner RWG, Humar A, Kandaswamy R, Najarian JS, Payne WD and Sutherland DER. Rapid discontinuation of steroids in living donor kidney transplantation: A pilot study. Am J Transplant 2001;1:278-83.

9. Grewal HP, Thistlethwaite JR, Loss GE, Bruce DS, Siegel CT, Cronin DC, Newell KA, Millis JM and Woodle ES. Corticosteroid cessation 1 week following renal transplantation using tacrolimus/mycophenolate mofetil based immunosuppression. Transplant Proc 1998;30: 1378-9.

10. Cole E, Landsberg D, Russell D, Zaltzman J, Kiberd B, Caravaggio C, Vasquez AR and Halloran P. A pilot study of steroid-free immunosuppression in the prevention of acute rejection in renal allograft recipients. Transplantation 2001;72:845-50.

11. Hooff JP van, Boots JMM, Duijnhoven EM van and Christiaans MHL. Dosing and management guidelines for tacrolimus in renal transplant patients. Transplant Proc 1999;31 (S7A): 54S-7.

12. Racusen LC, Solez K, Colvin RB, Bonsib SM, Castro MC, Cavallo T, Croker BP, Demetris AJ, Drachenberg CB, Fogo AB, Furness P, Gaber LW, Gibson IW, Glotz D, Goldberg JC, Grande JG, Halloran PF, Hansen HE, Hartley B, Hayry PJ, Hill CM, Hoffman EO, Hunsicker 
LG, Lindblad AS, Marcussen N, Mihatsch MJ, Nadasdy T, Nickerson P, Olsen TS, Papadimitriou JC, Randhawa PS, Rayner DC, Roberts I, Rose S, Rush D, Salinas-Madrigal L, Salomon DR, Sund S, Taskinen E, Trpkov K and Yamaguchi Y. The Banff 97 working classification of renal allograft pathology. Kidney Int 1999;55:713-23.

13. Ginsberg JM, Chang BS, Matarese RA and Garella S. Use of single voided urine samples to estimate quantitative proteinuria. N Engl J Med 1983;309:1543-6.

14. Shapiro R, Jordan ML, Scantlebury VP, Vivas C, Marsh JW, McCauley J, Johnston J, Randhawa P, Irish W, Gritsch HA, Naraghi R, Hakala TR, Fung JJ and Starzl TE. A prospective, randomized trial of tacrolimus/prednisone versus tacrolimus/prednisone/ mycophenolate mofetil in renal transplant recipients. Transplantation 1999;67:411-5.

15. Calconi $G$ and Vianello A for the Italian and Spanish Tacrolimus Study Group. One-year follow-up of a large European trial comparing dual versus triple tacrolimus-based immunosuppressive regimens following renal transplantation. Transplant Proc 2001;33:1021-4.

16. Squifflet J-P, Bäckman L, Claesson K, Dietl KH, Ekberg H, Forsythe JLR, Kunzendorf U, Heemann U, Land W, Morales JM, Mühlbacher F, Talbot D, Taube D, Tyden G, Hooff JP van, Schleibner S and Vanrenterghem Y for the European Tacrolimus-MMF Renal Study Group. Dose optimization of mycophenolate mofetil when administered with a low dose of tacrolimus in cadaveric renal transplant recipients. Transplantation 2001;72:63-9.

17. Chang RWS, Snowden S, Palmer A, Kwan JTC, Nicholson M, Kashi SH, Fernando ON, Perner F and Neild GH. European randomised trial of dual versus triple tacrolimus-based regimens for control of acute rejection in renal allograft recipients. Transpl Int 2001;14:384-90.

18. Matas A. Chronic rejection in renal transplant recipients - risk factors and correlates. Clin Transplant 1994;8:332-5.

19. Saase JLCM, Woude FJ van der, Thorogood J, Hollander AAMJ, Es LA van, Weening JJ, Bockel JH van and Bruijn JA. The relation between acute vascular and interstitial renal allograft rejection and subsequent chronic rejection. Transplantation 1995;59: 1280-5.

20. Madden RL, Mulhern JG, Benedetto BJ, O'Shea MH, Germain MJ, Braden GL, O'Shaughnessy J and Lipkowitz GS. Completely reversed acute rejection is not a significant risk factor for the development of chronic rejection in renal allograft recipients. Transpl Int 2000;13:344-50.

21. Ahsan N, Hricik D, Matas A, Rose S, Tomlanovich S, Wilkinson A, Ewell M, McIntosh M, Stablein D and Hodge E for the Steroid Withdrawal Group. Prednisone withdrawal in kidney transplant recipients on cyclosporin and mycophenolate mofetil - a prospective randomized study. Transplantation 1999;68:1865-74.

22. Vanrenterghem Y, Lebranchu Y, Hené R, Oppenheimer F and Ekberg H for the Steroid Dosing Study Group. Double-blind comparison of two corticosteroid regimens plus mycophenolate mofetil and cyclosporin for prevention of acute renal allograft rejection. Transplantation 2000; 70:1352-9.

23. Boots JMM, Duijnhoven EM van, Christiaans MHL, Wolffenbuttel BHR and Hooff JP van. Glucose metabolism in renal transplant recipients on tacrolimus: the effect of steroid withdrawal and tacrolimus trough level reduction. J Am Soc Nephrol 2002;13:221-7.

24. Undre NA, Hooff J van, Christiaans M, Vanrenterghem Y, Donck J, Heeman U, Kohnle M, Zanker W, Morales JM, Andrés A, Schäfer A and Stevenson P. Low systemic exposure to tacrolimus correlates with acute rejection. Transplant Proc 1999;31:296-8. 
25. Undre NA, Schäfer A and the European Tacrolimus Multicentre Renal Study Group. Factors affecting the pharmacokinetics of tacrolimus in the first year after renal transplantation. Transplant Proc 1998;30:1261-3.

26. Duijnhoven EM van, Boots JMM, Christiaans MHL, Stolk LML, Undre NA and Hooff JP van. Increase in tacrolimus trough levels after steroid withdrawal. Transpl Int 2003;16:721-5.

27. Ratcliffe PJ, Dudley CRK, Higgins RM, Firth JD, Smith B and Morris PJ. Randomised controlled trial of steroid withdrawal in renal transplant recipients receiving triple immunosuppression. Lancet 1996;348:643-8.

28. Sund S, Reisæter AV, Fauchald P, Bentdal $\varnothing$, Sundby Hall K and Hovig T. Living donor kidney transplants: a biopsy study 1 year after transplantation, compared with baseline changes and correlation to kidney function at 1 and 3 years. Nephrol Dial Transplant 1999;14:2445-54.

29. Terasaki PI, Koyama H, Cecka JM and Gjertson DW. The hyperfiltration hypothesis in human renal transplantation. Transplantation 1994;57:1450-4.

30. Alcalde G, Escallada R, Cotorruelo JG, Zubimendi JA, Francisco ALM de and Arias M. Disproportion between kidney graft and recipient size is the main predictor of long-term proteinuria. Transplant Proc 1997;29:127-8.

31. Nankivell BJ, Fenton-Lee CA, Kuypers DRJ, Cheung E, Allen RDM, O'Connell PJ and Chapman JR. Effect of histological damage on long-term kidney transplant outcome. Transplantation 2001;71:515-23. 


\section{Chapter 6}

\section{Glucose metabolism in renal transplant recipients on tacrolimus: the effect of steroid withdrawal and tacrolimus trough level reduction}

JMM Boots, EM van Duijnhoven, MHL Christiaans, BHR Wolffenbuttel, JP van Hooff, 


\section{Abstract}

The relative role of steroids and tacrolimus in the development of glucose metabolic disorders and hyperlipidemia after renal transplantation has not yet been clearly established. Therefore, glucose metabolism was prospectively evaluated by intravenous glucose tolerance test, as was lipid profile, in fifteen white non-diabetic renal transplant recipients three times: before and after steroid withdrawal, and after tacrolimus trough level reduction. After withdrawal of $10 \mathrm{mg}$ of prednisolone, insulin resistance decreased (fasting C-peptide, 0.99 to $0.77 \mathrm{nmol} / 1(P<0.0009)$; fasting insulin, 9.5 to $8.1 \mathrm{mU} / 1(P=0.09)$; insulin/glucose ratio, 1.85 to $1.45 \mathrm{mU} / \mathrm{mmol}(P=0.10)$ ) and lipid levels decreased (total cholesterol, 5.1 to $4.2 \mathrm{mmol} / \mathrm{l}(P=0.006)$; HDL cholesterol, 1.4 to $1.1 \mathrm{mmol} / 1$ $(P=0.01)$; LDL cholesterol, 3.0 to $2.5 \mathrm{mmol} / 1(P=0.15)$; triglycerides, 1.52 to $0.91 \mathrm{mmol} / 1 \quad(P=0.02)$. After tacrolimus trough level reduction from 9.5 to $6.4 \mathrm{ng} / \mathrm{ml}$, pancreatic $\beta$-cell secretion capacity improved (C-peptide secretion increased from 49.0 to $66.6 \mathrm{nmol} \times \mathrm{min} / 1(P=0.04)$ and insulin secretion increased from 1134 to $1403 \mathrm{mU} \times \mathrm{min} / \mathrm{l}(P=0.06))$. $\mathrm{HbA}_{1} \mathrm{c}$ improved also, from 5.9 to $5.3 \%(P=0.002)$. Lipids did not change. In conclusion, steroid withdrawal resulted in a decrease in insulin resistance and a reduction in lipids, and tacrolimus trough level reduction resulted in an improved pancreatic $\beta$-cell secretion capacity. Therefore, these therapeutic measurements may contribute to the reduction of the cardiovascular morbidity and mortality in renal transplant recipients. 


\section{Introduction}

Both post-transplant diabetes mellitus (PTDM) and hyperlipidemia contribute to cardiovascular mortality and graft failure after transplantation ${ }^{1-4}$.

PTDM occurs in up to $36 \%$ of transplant patients ${ }^{4-9}$. PTDM develops because of impairment of insulin secretion and/or increase of insulin resistance. Patients with an impaired $\beta$-cell function before transplantation are mainly at risk for developing PTDM during use of calcineurin inhibitors ${ }^{10,11}$. In dialysis patients, we were able to demonstrate that tacrolimus caused impaired insulin secretion, but had no influence on insulin resistance. However, insulin resistance has been suggested as an additional mechanism responsible for the development of PTDM during maintenance therapy with tacrolimus in renal transplant recipients ${ }^{5}$. The coadministration of steroids might well have been the cause of this increased insulin resistance ${ }^{11,12}$.

Hyperlipidemia occurs in many patients after solid organ transplantation ${ }^{13}$. The type of calcineurin inhibitor plays a role in the hyperlipidemia: lipid profiles in renal transplant recipients treated with tacrolimus were better than those in patients treated with cyclosporin ${ }^{14,15}$.

Aside from causing other side effects ${ }^{16}$, steroids play a role in the development of both PTDM and post-transplant hyperlipidemia ${ }^{17,18}$. Several investigators have shown that steroid withdrawal is safe in a large proportion of transplant recipients ${ }^{15,19}$. Since then, improvement in lipid profile after steroid withdrawal on both cyclosporin and tacrolimus-based immunosuppression has been described in liver transplant recipients ${ }^{20}$. In renal transplant recipients, lipid profile improvement after steroid withdrawal has been reported only in patients on cyclosporin ${ }^{21,22}$, but not in patients on tacrolimus. In renal transplant recipients on tacrolimus, it is unknown what the effects are of steroid withdrawal on glucose metabolism. Not only the use of steroids, but also tacrolimus exposure are important factors that influence glucose metabolism, because it is a well known observation that PTDM disappears in a high proportion of the patients after tacrolimus dose reduction ${ }^{23}$. The relative role of steroids and tacrolimus levels in the development of glucose metabolic disorders and hyperlipidemia is unknown. Therefore, we prospectively evaluated glucose metabolism and lipid profile in renal transplant recipients on tacrolimus-based immunosuppression three times: before and after steroid withdrawal, and subsequently, after tacrolimus trough level reduction. 


\section{Material and methods}

\section{Patients}

Renal transplant recipients on tacrolimus and prednisolone were eligible to participate in the study if they were $>16$ years of age and scheduled for steroid withdrawal between three and 12 months post-transplantation after obtaining a normal ACTH stimulation test. Additional use of mycophenolate mofetil (MMF) was allowed. Exclusion criteria were as follows: contraindications for steroid withdrawal (defined as severe acute rejection (grade 2 or more in the Banff classification) or steroid-resistant rejection), use of steroids for comorbidity, preexisting diabetes mellitus or development of PTDM prior to the first test (defined as a need for oral blood glucose-lowering medication or insulin), and underlying disease known to interfere with the hypothalamus-pituitary-adrenal axis or glucose metabolism. Written informed consent was obtained from all patients. The medical ethical committee of our hospital approved the protocol of steroid withdrawal.

Fifteen white patients (13 men, two women) participated in the study. Their median age was 58 years (range 16 to 75 years) and their median body mass index (BMI) was $23.8 \mathrm{~kg} / \mathrm{m}^{2}$ (range 20.4 to $27.5 \mathrm{~kg} / \mathrm{m}^{2}$ ). There were 13 first transplantations and two retransplantations; 12 patients had a cadaveric donor, two a living unrelated donor, and one a living related donor.

\section{Immunosuppression}

All patients started tacrolimus $(0.1 \mathrm{mg} / \mathrm{kg}$ orally twice daily) within 12 hours before transplantation. Further dosage adjustments were made according to whole blood 12-hours trough levels (Imx, Abbott, Hoofddorp, The Netherlands). The target range was 15 to $20 \mathrm{ng} / \mathrm{ml}$ in week 1 and 2 post-transplantation, and 10 to $15 \mathrm{ng} / \mathrm{ml}$ in week 3 and 4 . Thereafter, the dose was gradually tapered to target levels below $10 \mathrm{ng} / \mathrm{ml}$. Immediately after transplantation, 14 patients were also treated with MMF (1000 mg/day). At the time of the first and second intravenous glucose tolerance test (iv-GTT), nine patients were still using MMF. At the time of the third tests, four patients were still using MMF. The prednisolone dose was 20 $\mathrm{mg}$ in week 1 and 2, and $15 \mathrm{mg}$ in week 3 and 4 after transplantation. Thereafter, the dose was $10 \mathrm{mg}$ until withdrawal. Withdrawal was started at a median of 185 days (range: 81 to 358 days) after transplantation. The prednisolone dosage was reduced to $5 \mathrm{mg} /$ day for one week and $2.5 \mathrm{mg} /$ day for a second week; thereafter it was stopped. For each patient, we aimed for the same individual tacrolimus trough level before and after steroid withdrawal. After the second test, tacrolimus dosages were adjusted gradually to reach target trough levels of 5 to $7 \mathrm{ng} / \mathrm{ml}$. 


\section{Glucose metabolism}

The tests were performed in the morning, after a 12-hour overnight fast. Tacrolimus was ingested after completion of the tests. The other medication was ingested at the usual time of day. In case of a stressful event (e.g., surgery or infection), the test was postponed for at least three weeks.

Glucose $50 \%$ ( $0.5 \mathrm{~g} / \mathrm{kg}$ body weight) was administered intravenously for two to three minutes. Blood samples for measurement of whole blood glucose, Cpeptide, and insulin were taken from the opposite arm at $\mathrm{t}=-15,0,5,10,15,20$, 30, 40, 50, and 60 minutes. Insulin sensitivity index (glucose disappearance rate $=\mathrm{k}_{\mathrm{G}}$ ) was calculated by linear regression from the log-transformed glucose values of $\mathrm{t}=10$ to 30 minutes. $\mathrm{A} \mathrm{k}_{\mathrm{G}}$ value above $1.2 \mathrm{mmol} / \mathrm{l}$ per $\mathrm{min}$ was considered normal24,25. C-peptide and insulin secretion (increment), i.e., the secretion response to a glucose load, were calculated as area under the curve using a linear trapezoidal technique from the serum value at each time point after subtraction of the $t=0$ value. Insulin resistance was calculated by using the fasting insulin/glucose ratio and the homeostasis model assessment (HOMA-R: fasting glucose $(\mathrm{mmol} / \mathrm{l}) \times$ fasting insulin $(\mathrm{mU} / \mathrm{l}) / 22.5)^{26,27}$. Mean blood pressure, renal function (Cockcroft-Gault formula), hemoglobin, and $\mathrm{HbA}_{1} \mathrm{c}$ were measured at the time of the iv-GTTs. The use of drugs that might interfere with glucose metabolism (antihypertensive drugs (especially beta-blockers and diuretics), oral contraceptives, phenytoin, and pentamidine) and lipids were monitored.

During each visit to the outpatient clinic, urine was examined for glucosuria (by dipstick). When glucosuria was detected, whole blood glucose was examined. When no glucosuria was detected, whole blood glucose was evaluated at least every three months. PTDM was diagnosed when glucose values were abnormal $(>6.1 \mathrm{mmol} / \mathrm{l}$ in the fasting state or $>7.8 \mathrm{mmol} / \mathrm{l}$ in the non-fasting state), for two or more different samples and without any other explanation, such as additional high-dose steroids, infection, or operative stress.

For the measurement of glucose in whole blood, the CX7 (Beckman, Mijdrecht, The Netherlands) was used. For C-peptide and insulin, IRMA (Autodelfia, Wallac, Turku, Finland) was used. For $\mathrm{HbA}_{1} \mathrm{c}$, HPLC (Variant 2, Biorad, Hercules, CA). Normal reference values from our laboratory were 3.1 to $6.1 \mathrm{mmol} / 1$ for fasting glucose, 1.0 to $25.0 \mathrm{mU} / 1$ for fasting insulin, 0.12 to $1.20 \mathrm{nmol} / 1$ for fasting Cpeptide levels, and $4.4 \%$ to $6.2 \%$ for $\mathrm{HbA}_{1} \mathrm{c}$.

\section{Lipid profiles}

Fasting blood samples for lipid measurements were taken at the time of the iv-GTTs. The LX (Beckman) was used to determine lipid levels: total cholesterol (Roche Diagnostics, Almere, The Netherlands), HDL cholesterol (Roche Diagnostics), LDL cholesterol (LDL precipitating reagent, Merck, Amsterdam,

The Netherlands), and triglycerides (Synchron, Beckman). Normal reference 
values from our laboratory were 4.1 to $6.4 \mathrm{mmol} / 1$ for total cholesterol, 0.6 to $1.9 \mathrm{mmol} / 1$ for HDL cholesterol, 3.0 to $4.5 \mathrm{mmol} / 1$ for LDL cholesterol, and 0.80 to $1.94 \mathrm{mmol} / 1$ for triglycerides.

Throughout the study, no changes in lipid-lowering drugs were allowed.

\section{Statistics}

For statistical analysis, SPSS version 10.0 for Windows (SPSS Inc., Chicago, IL) was used. To compare glucose metabolism before and after steroid withdrawal, as well as before and after tacrolimus trough level reduction, the Wilcoxon matchedpairs signed rank sum test was performed. For correlations between the different parameters, the Spearman rho rank correlation coefficient was used. Statistical significance was defined as $P<0.05$. Unless indicated otherwise, data are described as median (range).

\section{Results}

Table 6.1 shows median levels of BMI, mean blood pressure, renal function, hemoglobin levels, tacrolimus trough levels, parameters of glucose metabolism, and lipid profiles before and after steroid withdrawal, and after tacrolimus trough level reduction.

\section{Patients and immunosuppression}

All patients completed the study. No person was treated for rejection. BMI and mean blood pressure did not change significantly. Many patients were treated with antihypertensive drugs: beta-blockers $(n=11)$, diuretics $(n=2)$, calcium-antagonists $(\mathrm{n}=9-12$, at various time points), angiotensin-converting enzyme-inhibitors $(n=3-5)$, and vasodilators $(n=3-4)$. There were no significant changes in the use of the various antihypertensive drugs. Nobody used other drugs that might have interfered with glucose metabolism. Hemoglobin decreased by $5 \%(P=0.04)$ after steroid withdrawal, and did not change after tacrolimus trough level reduction. Creatinine clearance did not change significantly after steroid withdrawal, but increased by $16 \%(P=0.002)$ after tacrolimus trough level reduction. After steroid withdrawal, tacrolimus dose was reduced in 11 patients and remained unchanged in the other four patients. Despite the dose reduction, there was a (statistically) non-significant rise in tacrolimus trough level (Figure 6.1). Between the second and third tests, trough level decreased by 33\% ( $P=0.004)$, as intended (Figure 6.1). 
Table 6.1 Clinical and laboratory parameters before and after steroid withdrawal, and after tacrolimus trough level reduction

\begin{tabular}{|c|c|c|c|c|c|}
\hline & $\begin{array}{c}\text { Before steroid } \\
\text { withdrawal }\end{array}$ & $\begin{array}{c}\text { After steroid } \\
\text { withdrawal }\end{array}$ & $\begin{array}{l}\text { After trough } \\
\text { level reduction }\end{array}$ & $\begin{array}{c}\text { Steroid } \\
\text { withdrawal } \\
P\end{array}$ & $\begin{array}{c}\text { Trough level } \\
\text { reduction } \\
P\end{array}$ \\
\hline \multicolumn{6}{|l|}{ Patients and immunosuppression } \\
\hline BMI (kg/m2) & $23.8(20.4-27.5)$ & $24.0(19.6-27.7)$ & $23.7(21.0-29.1)$ & 0.76 & 0.07 \\
\hline Mean blood pressure $(\mathrm{mmHg})$ & $103(95-123)$ & $102(88-113)$ & $102(83-123)$ & 0.27 & 0.97 \\
\hline Creatinine clearance $(\mathrm{ml} / \mathrm{min})$ & $61.3(32.4-83.3)$ & $56.6(35.5-95.8)$ & $65.5(42.3-96.6)$ & 0.61 & 0.002 \\
\hline Hemoglobin level (mmol/l) & $8.7(7.3-10.4)$ & $8.3(6.8-9.7)$ & $8.7(7.2-9.8)$ & 0.04 & 0.10 \\
\hline Tacrolimus trough level (ng/ml) & $8.2(5.9-17.3)$ & $9.5(6.7-18.4)$ & $6.4(5.0-10.5)$ & 0.78 & 0.004 \\
\hline \multicolumn{6}{|l|}{ Glucose metabolism } \\
\hline Fasting glucose (mmol/l) & $5.3(4.2-6.5)$ & $5.4(4.2-6.7)$ & $5.2(4.3-6.6)$ & 0.39 & 0.23 \\
\hline Fasting C-peptide (nmol/l) & $0.99(0.67-1.95)$ & $0.77(0.46-1.25)$ & $0.76(0.38-1.10)$ & 0.0009 & 0.75 \\
\hline Fasting insulin (mU/l) & $9.5(1.6-50.0)$ & $8.1(4.2-37.0)$ & $7.6(3.0-13.0)$ & 0.09 & 0.87 \\
\hline HOMA-R (mmol/l x mU/l) & $2.20(0.38-11.56)$ & $1.88(1.03-8.72)$ & $1.83(0.69-3.06)$ & 0.17 & 0.87 \\
\hline Insulin/glucose ratio $(\mathrm{mU} / \mathrm{mmol})$ & $1.85(0.30-9.62)$ & $1.45(0.75-6.98)$ & $1.52(0.58-2.45)$ & 0.10 & 1.00 \\
\hline $\mathrm{K}_{\mathrm{G}}(\mathrm{mmol} / \mathrm{l}$ per min $)$ & $1.33(0.93-2.53)$ & $1.63(0.58-3.95)$ & $1.57(0.54-5.92)$ & 0.65 & 0.14 \\
\hline C-peptide secretion (nmol x min/l) & $57.2(39.0-150.1)$ & $49.0(33.4-132.7)$ & $66.6(37.7-176.0)$ & 0.06 & 0.04 \\
\hline Insulin secretion (mU x min/l) & $1141(597-4534)$ & $1134(473-4105)$ & $1403(823-5865)$ & 0.13 & 0.06 \\
\hline HbA1c $(\%)$ & $6.0(5.3-7.2)$ & $5.9(4.8-6.5)$ & $5.3(4.8-6.1)$ & 0.17 & 0.002 \\
\hline \multicolumn{6}{|l|}{ Lipid profile } \\
\hline Total cholesterol (mmol/l) & $5.1(3.8-6.7)$ & $4.2(3.4-6.0)$ & $4.1(3.5-6.0)$ & 0.006 & 0.62 \\
\hline HDL-cholesterol (mmol/l) & $1.4(1.0-2.1)$ & $1.1(0.9-1.7)$ & $1.2(0.9-1.7)$ & 0.01 & 0.95 \\
\hline LDL-cholesterol (mmol/l) & $3.0(1.9-3.9)$ & $2.5(1.9-3.7)$ & $2.6(1.8-3.9)$ & 0.15 & 0.44 \\
\hline LDL/HDL ratio & $1.9(1.5-2.8)$ & $2.1(1.8-3.0)$ & $2.4(1.3-3.4)$ & 0.16 & 0.45 \\
\hline Triglycerides (mmol/l) & $1.52(1.21-2.07)$ & $0.91(0.57-1.96)$ & $0.91(0.57-1.96)$ & 0.02 & 0.33 \\
\hline
\end{tabular}

Median (and ranges) for all 15 patients. For significances $(P)$ of steroid withdrawal, the results before and after steroid withdrawal were compared, for significances $(P)$ of trough level reduction, the results after steroid withdrawal and the results after tacrolimus trough level reduction were compared

\section{Glucose metabolism}

Glucose metabolism was assessed by iv-GTT at a median of 20 days (range 13 to 35 days) before steroid withdrawal, 68 days (39 to 96 days) after complete cessation of steroids, and 307 days (163 to 345 days) thereafter (after tacrolimus trough level reduction). None of the patients developed hyperglycemia.

Figure 6.1, 6.2 and 6.3 show the median levels of the various parameters of glucose metabolism before and after steroid withdrawal, and after tacrolimus trough level reduction. Figure 6.1 shows fasting glucose and $\mathrm{HbA}_{1} \mathrm{c}$, Figure 6.2 
shows fasting parameters associated with insulin resistance, and Figure 6.3 shows $\mathrm{k}_{\mathrm{G}}$ and stimulated parameters associated with pancreatic $\beta$-cell secretion capacity. After steroid withdrawal, fasting C-peptide levels decreased significantly by $22 \%$ $(P=0.0009)$, fasting insulin levels and insulin/glucose ratio decreased as well $(P=0.09$ and 0.10 , respectively). C-peptide secretion decreased by $14 \%(P=0.06)$. There was no significant change in any other parameter of glucose metabolism after steroid withdrawal.

After tacrolimus trough level reduction, C-peptide secretion increased significantly by $36 \%(P=0.04)$. A similar increase was observed for insulin secretion $(P=0.06)$. $\mathrm{HbA}_{1} \mathrm{c}$ decreased significantly by $10 \%(P=0.002)$. Insulin resistance parameters did not change.

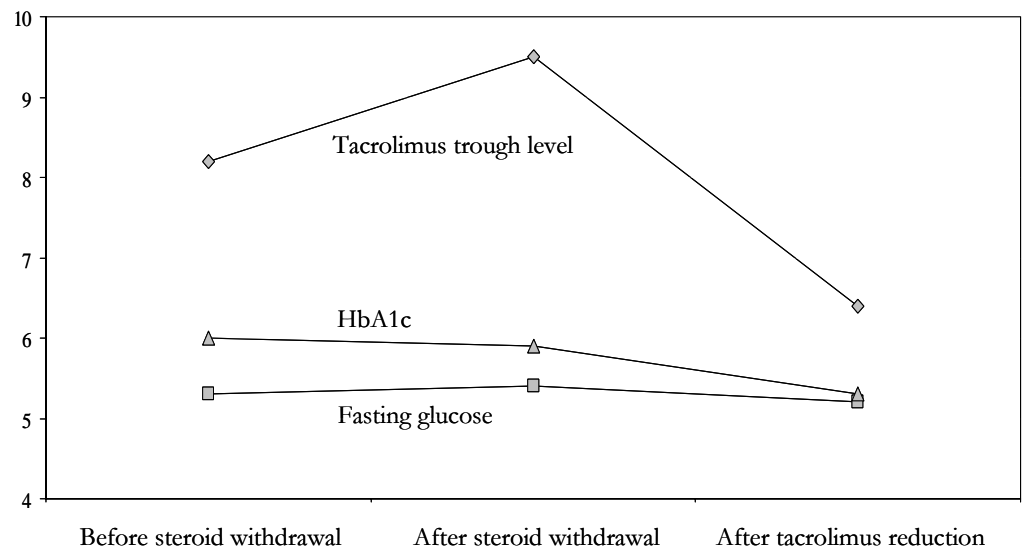

Figure 6.1 Median tacrolimus trough level, fasting glucose, and $\mathrm{HbA}_{1} \mathrm{c}$ levels before and after steroid withdrawal, and after tacrolimus trough level reduction. Tacrolimus trough level ( $\mathrm{ng} / \mathrm{ml}), \mathrm{HbA}_{1} \mathrm{c}(\%)$, fasting glucose ( $\left.\mathrm{mmol} / \mathrm{l}\right)$.

\section{Lipid profile}

Before and after steroid withdrawal, two patients were treated with $10 \mathrm{mg}$ simvastatin. After tacrolimus trough level reduction, the dosage of simvastatin in one of these patients was increased. Moreover, three additional patients were treated with lipid-lowering drugs. These four patients were therefore excluded from analysis.

After steroid withdrawal, there was a decrease in total cholesterol by $11 \%$ $(P=0.006)$, HDL-cholesterol by $21 \%(P=0.01)$ and triglycerides by $25 \%(P=0.02)$, a decrease of LDL-cholesterol by $9 \%(P=0.15)$, and an increase of LDL/HDL ratio by $12 \%(P=0.16)$.

After tacrolimus dose reduction, there were no significant changes in lipids. 


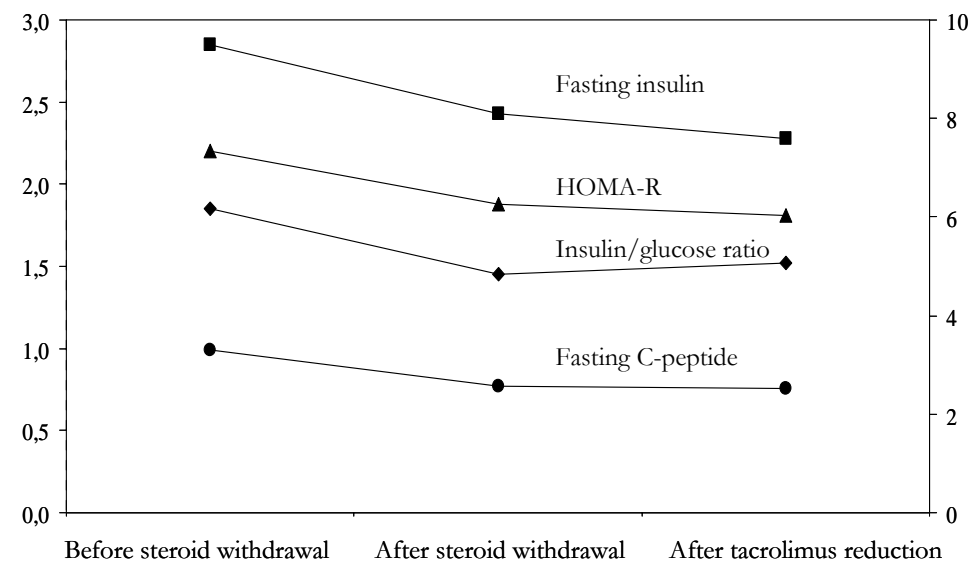

Figure 6.2 Median fasting parameters of glucose metabolism associated with insulin resistance before and after steroid withdrawal, and after tacrolimus trough level reduction. Right $\mathrm{y}$-axis: fasting insulin (mU/l). Left y-axis: fasting C-peptide (nmol/l), HOMA-R $\left(\mathrm{mmol} / \mathrm{l}^{*} \mathrm{mU} / \mathrm{l}\right)$, and insulin glucose ratio $(\mathrm{mU} / \mathrm{mmol})$.

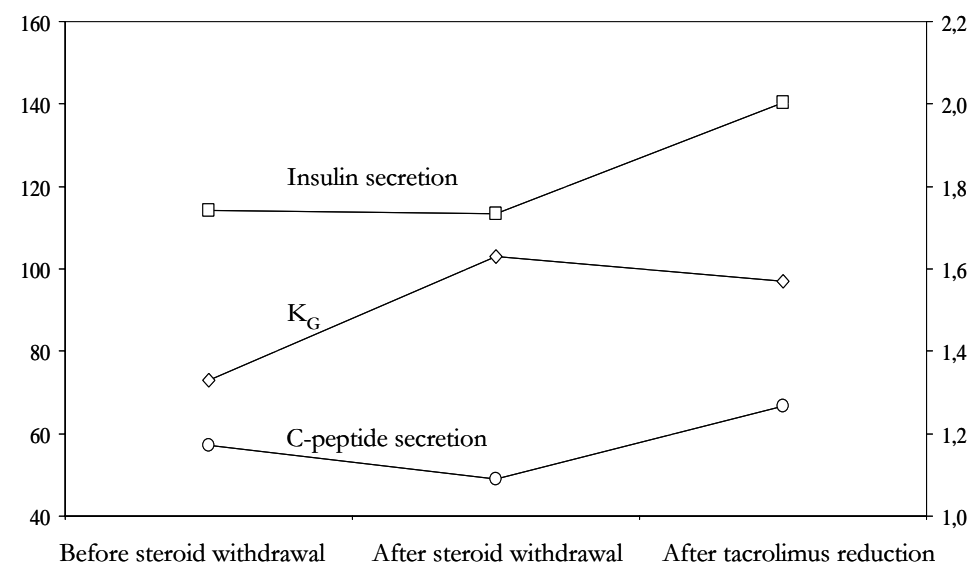

Figure 6.3 Median fasting $\mathrm{k}_{\mathrm{G}}$ and parameters of glucose metabolism associated with pancreatic $\beta$ cell secretion capacity before and after steroid withdrawal, and after tacrolimus trough level reduction.

Right y-axis: $\mathrm{K}_{\mathrm{G}}\left(\mathrm{mmol} / \mathrm{l}\right.$ per min). Left y-axis: C-peptide secretion $\left(\mathrm{nmol}{ }^{*} \min / \mathrm{l}\right)$ and insulin secretion $\left(\mathrm{mU}^{*} \mathrm{~min} / 1\right.$ divided by 10$)$. 


\section{Correlations between tacrolimus trough level and parameters of glucose metabolism}

Tacrolimus trough level correlated significantly with $\mathrm{k}_{\mathrm{G}}$ (rho=-0.362, $\left.P=0.015\right)$, and with $C$-peptide secretion (rho $=-0.311, P=0.037$ ), but not with any of the other parameters of glucose metabolism.

\section{Discussion}

In this prospective study in white renal transplant recipients the relative roles of steroids and tacrolimus on glucose metabolism and lipid profiles were evaluated.

After steroid withdrawal, fasting C-peptide decreased significantly. C-peptide is almost completely cleared by the kidney ${ }^{28}$. Renal function did not change significantly. Therefore, improvement in renal function could not have been responsible for the decrease in C-peptide. From this observation, one can conclude that use of steroids causes an increase in insulin resistance in patients on tacrolimus, confirming the data in cyclosporine patients ${ }^{12}$.

After steroid withdrawal, $C$-peptide secretion also decreased $(P=0.06)$. It is very unlikely that this decrease is due to a direct effect of steroid withdrawal on $\beta$-cell production. In our opinion, this is due to an indirect effect of increased tacrolimus trough levels. We have observed that tacrolimus trough levels (with unchanged dose) increase after steroid withdrawal ${ }^{29}$. This increase of trough levels does not occur in all patients, but in 40 to $60 \%$ of the patients. Due to the reduction in tacrolimus dose in $73 \%$ of the patients, the increase of tacrolimus trough level in this study was not statistically significant. But despite dose reductions, tacrolimus trough levels increased by more then $20 \%$ in four patients (range 43 to $65 \%$ ). In three of the latter patients C-peptide secretion decreased by 25 to $46 \%$.

In the second part of the study, tacrolimus trough level was reduced, resulting in a significant increase in stimulated C-peptide secretion. Insulin resistance did not change. Thus, pancreatic $\beta$-cell secretion capacity increases when tacrolimus trough levels are lowered, confirming the reversibility of $\beta$-cell dysfunction caused by tacrolimus ${ }^{11,23,30}$. This occurs even within the normal range for maintenance therapy with tacrolimus: a 33\% reduction in the tacrolimus trough level resulted in a $36 \%$ increase in median $\beta$-cell secretion capacity. The period between the iv-GTT before and after trough level reduction was approximately ten months because patients were generally seen only once every two to three months and a gradual decrease in tacrolimus trough levels to $5 \mathrm{ng} / \mathrm{ml}$ was desired. BMI, blood pressure and the use of antihypertensive drugs did not change in this period. We cannot completely rule out a spontaneous improvement of pancreatic $\beta$-cell secretion capacity in this period. However, given the correlation found between tacrolimus trough level and pancreatic $\beta$-cell function in both this study and our previous studies ${ }^{11,30}$ and observations of improved glucose metabolism after 
tacrolimus dose reduction ${ }^{30}$, it is more likely that the reduced tacrolimus trough levels explain at least part of the improvement.

Before and after steroid withdrawal, concomitant use of MMF was the same, but before and after tacrolimus trough level reduction, five patients discontinued MMF. Although there are no descriptions of alterations in glucose metabolism or lipid profile due to the use of MMF, we compared changes in parameters of glucose metabolism after steroid withdrawal and after trough level reduction separately for patients using MMF and those not using MMF. The changes in both groups were similar to changes for all patients.

After tacrolimus trough level reduction, $\mathrm{HbA}_{1} \mathrm{c}$ decreased significantly. This is an important finding because an association between $\mathrm{HbA}_{1} \mathrm{c}$ levels and risk of cardiovascular morbidity and mortality has been described in nondiabetic patients ${ }^{31}$. In our study, hemoglobin levels did not change significantly after trough level reduction. Therefore, it seems unlikely that the decrease in $\mathrm{HbA}_{1} \mathrm{c}$ in our study can be explained by an increase in hemoglobin levels ${ }^{32}$. $\mathrm{HbA}_{1} \mathrm{c}$ levels in patients with renal insufficiency and patients after kidney transplantation correlate well with mean capillary blood glucose levels; therefore, the improvement in renal function in our patients does not seem to explain the decrease in $\mathrm{HbA}_{1} \mathrm{c}$ either ${ }^{33}$. Furthermore, fasting glucose levels did not change in our study; therefore, reduced post-prandial glucose levels must have caused the decrease in $\mathrm{HbA}_{1} \mathrm{c}$. In the DECODE study ${ }^{34}$, abnormal blood glucose two hours after an oral glucose tolerance test was associated with an increased mortality risk by a factor 2.0 for men and 2.8 for women. An increased risk of cardiovascular disease has also been shown in studies comparing normal and increased post-prandial blood glucose levels ${ }^{35}$. Consequently, tacrolimus trough level reduction, which results in lower post-prandial glucose and $\mathrm{HbA}_{1} \mathrm{c}$ levels might reduce cardiovascular morbidity and mortality, not only in patients with PTDM but also in non-diabetic patients.

Due to a violation of protocol, lipid-lowering drugs were changed in four patients. However, between the first and second test, where relevant changes in lipids occurred, lipid-lowering drugs were unchanged. To exclude bias due to the exclusion of patients we also compared lipid profiles before and after steroid withdrawal for all 15 patients. The results of this analysis were the same. The changes in lipids after steroid withdrawal, i.e., a decrease in all lipids, are very similar to the changes described after steroid withdrawal in nondiabetic renal transplant recipients on cyclosporin-based immunosuppression ${ }^{22}$. The reductions in total cholesterol, LDL-cholesterol, and triglycerides are obviously beneficial. The extent to which these beneficial changes are counterbalanced by the concomitant decrease in HDL is unclear. We found no indications that decreasing tacrolimus trough levels influenced lipid profiles, indicating that tacrolimus does not influence lipids. However, a decrease in tacrolimus trough level by 33\% did result in a significant improvement in renal function of approximately $16 \%$. This could be of importance because studies in cyclosporin-patients have shown that 
renal function in the first year after transplantation has a large impact on longterm graft survival ${ }^{36}$.

Our findings support our current policy for patients who develop PTDM on tacrolimus-based immunosuppression: at first, steroid withdrawal, while maintaining therapeutic tacrolimus trough levels of approximately 10 to $15 \mathrm{ng} / \mathrm{ml}$ for four to six weeks after transplantation to avoid acute rejection. After this period, tacrolimus trough levels are reduced to 5 to $7 \mathrm{ng} / \mathrm{ml}$. This often results in a reduction in, or the cessation of, insulin therapy, or even in the disappearance of PTDM $^{15}$.

In summary, steroid withdrawal in white renal transplant recipients on tacrolimusbased immunosuppression leads to a general improvement in glucose metabolism, due to a decrease in insulin resistance, as well as to a reduction in lipids, and tacrolimus trough level reduction improves glucose metabolism by increasing pancreatic $\beta$-cell secretion capacity. 


\section{References}

1. Massy ZA and Kasiske BL. Post-transplant hyperlipidemia: Mechanisms and management. J Am Soc Nephrol 1996;7:971-7.

2. Aakhus S, Dahl K and Widerøe TE. Cardiovascular morbidity and risk factors in renal transplant patients. Nephrol Dial Transplant 1999;14:648-54.

3. Kasiske BL. Cardiovascular disease after renal transplantation. Semin Nephrol 2000;20:176-87.

4. Jindal RM, Sidner RA and Milgrom ML. Post-transplant diabetes mellitus. The role of immunosuppression. Drug Saf 1997;16:242-57.

5. Weir MR and Fink JC. Risk for posttransplant diabetes mellitus with current immunosuppressive medications. Am J Kidney Dis 1999;34:1-13.

6. Krentz AJ, Dmitrewski J, Mayer D and Natrass M. Effects of immunosuppressive agents on glucose metabolism. Clin Immunother 1995;4:103-23.

7. Mayer AD, Dmitrewski J, Squifflet J-P, Besse T, Grabensee B, Klein B, Eigler FW, Heemann U, Pichlmayr R, Behrend M, Vanrenterghem Y, Donck J, Hooff J van, Christiaans M, Morales JM, Andres A, Johnson RWG, Short C, Buchholz B, Rehmert N, Land W, Schleibner S, Forsythe JLR, Talbot D, Neumayer H-H, Hauser I, Ericzon B-G, Brattström C, Claesson K, Mühlbacher F and Pohanka E. Multicenter randomized trial comparing tacrolimus (FK 506) and cyclosporin in the prevention of renal allograft rejection. A report of the European Tacrolimus Multicenter Renal Study Group. Transplantation 1997;64:436-43.

8. Pirsch JD, Miller J, Deierhoi MH, Vincenti F and Filo RS for the FK506 Kidney Transplant Study Group. A comparison of tacrolimus (FK506) and cyclosporine for immunosuppression after cadaveric renal transplantation. Transplantation 1997;63:977-83.

9. Knoll GA and Bell RC. Tacrolimus versus cyclosporin for immunosuppression in renal transplantation: Meta-analysis of randomised trials. Br Med J 1999;318:1104-7.

10. Nam JH, Mun JI, Kim SI, Kang SW, Choi KH, Park K, Ahn CW, Cha BS, Song YD, Lim SK, Kim KR, Lee HC and Huh KB. B-cell dysfunction rather than insulin resistance is the main contributing factor for the development of postrenal transplantation diabetes mellitus. Transplantation 2001;71:1417-23.

11. Duijnhoven EM van, Boots JMM, Christiaans MHL, Wolffenbuttel BHR and Hooff JP van. Influence of tacrolimus on glucose metabolism before and after renal transplantation: a prospective study. J Am Soc Nephrol 2001;12:583-8.

12. Ekstrand A, Ahonen J, Gronhagen-Riska C and Groop L. Mechanisms of insulin resistance after kidney transplantation. Transplantation 1989;48:563-8.

13. Jindal RM. Post-transplant hyperlipidemia. Postgrad Med J 1997;73:785-93.

14. McCune TR, Thacker LR II, Peters TG, Mulloy L, Rohr MS, Adams PA, Yium J, Light JA, Pruett T, Gaber AO, Selman SH, Jonsson J, Hayes JM, Wright FH jr, Armata T, Blanton J and Burdick JF. Effects of tacrolimus on hyperlipidemia after successful renal transplantation. A Southeastern Organ Procurement Foundation Multicenter Clinical Study. Transplantation 1998;65:87-92.

15. Boots JMM, Duijnhoven EM, Christiaans MHL, Nieman FHM, Suylen RJ van and Hooff JP van. Single center experience with tacrolimus versus cyclosporin-Neoral in renal transplant recipients. Transpl Int 2001;14:370-83. 
16. Veenstra DL, Best JH, Hornberger J, Sullivan SD and Hricik DE. Incidence and long-term cost of steroid-related side effects after renal transplantation. Am J Kidney Dis 1999;33:829-39.

17. Fernández-Miranda C, Guijarro C, Calle A de la, Loinaz C, Gonzalez-Pinto I, GomezIzquierdo T, Larumbe S, Moreno $\mathrm{E}$ and Palacio A del. Lipid abnormalities in stable liver transplant recipients--Effects of cyclosporin, tacrolimus, and steroids. Transpl Int 1998;11: $137-42$.

18. Pirsch JD, d'Alessandro AM, Sollinger HW, Knechtle SJ, Reed A, Kalayoglu M and Belzer FO 1. Hyperlipidemia and transplantation: Etiologic factors and therapy. J Am Soc Nephrol 1992;2(S12):S238-42.

19. Shapiro R, Jordan ML, Scantlebury VP, Vivas C, Gritsch HA, Corry RJ, Egidi F, McCauley J, Ellis D, Gilboa $\mathrm{N}$ et al. The superiority of tacrolimus in renal transplant recipients -- the Pittsburgh experience. Clin Transplant 1995;9:199-205.

20. McDiarmid SV, Farmer DA, Goldstein LI, Martin P, Vargas J, Tipton JR, Simmons F and Busuttil RW. A randomized prospective trial of steroid withdrawal after liver transplantation. Transplantation 1995;60:1443-50.

21. Hricik DE, Bartucci MR, Moir EJ, Mayes JT and Schulak JA. Effects of steroid withdrawal on posttransplant diabetes mellitus in cyclosporine-treated renal transplant recipients. Transplantation 1991;51:374-7.

22. Schulak JA and Hricik DE. Steroid withdrawal after renal transplantation. Clin Transplant 1994;8:211-6.

23. Shapiro R, Jordan M, Scantlebury V, Fung J, Jensen C, Tzakis A, McCauley J, Carroll P, Ricordi C, Demetris AJ, Mitchell S, Jain A, Iwaki Y, Kobayashi M, Reyes J, Todo S, Hakala TR, Simmons RL and Starzl TE. FK 506 in clinical kidney transplantation. Transplant Proc 1991;23:3065-7.

24. Elmer DS, Hathaway DK and Gaber AO. Monitoring of pancreas allograft function with glucose disappearance rate (KG): calculation and interpretation. J Transplant Coordination 1994;4:7-11.

25. Bergman RN, Finegood DT and Ader M. Assessment of insulin sensitivity in vivo. Endocrine Rev 1985;6:45-86.

26. Matthews DR, Hosker JP, Rudenski AS, Naylor BA, Treacher DF and Turner RC. Homeostasis model assessment: Insulin resistance and beta-cell function from fasting plasma glucose and insulin concentrations in man. Diabetologia 1985;28:412-9.

27. Avignon A, Boegner C, Mariano-Goulart D, Colette C and Monnier L. Assessment of insulin sensitivity from plasma insulin and glucose in the fasting or post oral glucose-load state. Int J Obes Relat Metab Disord 1999;23:512-7.

28. Greenspan FS and Baxter JD. Basic \& Clinical Endocrinology. (4th ed.) Nowalk: Appleton \& Lange, 1994:573.

29. Duijnhoven EM van, Boots JMM, Christiaans MHL, Stolk LML, Undre NA and Hooff JP van. Increase in tacrolimus trough levels after steroid withdrawal. Transpl Int 2003;16:721-5.

30. Duijnhoven EM van, Christiaans MHL, Boots JMM, Nieman FHR, Wolffenbuttel BHR and Hooff JP van. Glucose metabolism in the first 3 years after renal transplantation in patients on tacrolimus versus cyclosporine-based immunosuppression. J Am Soc Nephrol 2002;13:213-20.

31. Khaw K, Wareham N, Luben R, Bingham S, Oakes S, Welch A and Day N. Glycated haemoglobin, diabetes, and mortality in men in Norfolk cohort of European Prospective Investigation of Cancer and Nutrition (EPIC Norfolk). Br Med J 2001;322:15-8. 
32. Nakao T, Matsumoto H, Okada T, Han M, Hidaka H, Yoshino M, Shino T, Yamada C and Nagaoka Y. Influence of erythropoietin treatment on hemoglobin A1c levels in patients with chronic renal failure on hemodialysis. Intern Med 1998;37:836-0.

33. Morgan L, Marenah CB, Jeffcoate WJ and Morgen AG. Glycated proteins as indices of glycemic control in diabetic patients with chronic renal failure. Diabet Med 1996;13:514-9.

34. The DECODE study group. European diabetes epidemiology group: Glucose tolerance and mortality: comparison of WHO and American Diabetes Association diagnostic criteria. Diabetes Epidemiology: Collaborative analysis of diagnostic criteria in Europe. Lancet 1999; 354:617-21.

35. Balkau B, Pyorala M, Shipley MJ, Forthan A, Jarret RJ and Eschwege E. High blood glucose concentration is a risk factor for mortality in middle-aged nondiabetic men - 20-year follow-up in the Whitehall Study, the Paris Prospective Study and the Helsinki Policemen Study. Diabetes Care 1998;21:360-7.

36. Kuo PC, Johnson LB, Schweitzer EJ, Alfrey EJ, Waskerwitz J and Bartlett ST. Utilization of the older donor for renal transplantation. Am J Surg 1996;172:551-7. 


\section{Chapter 7}

\section{Improved cardiovascular risk profile and renal function in renal transplant patients after randomized conversion from cyclosporin to tacrolimus}

MA Artz, JMM Boots, G Ligtenberg, JI Roodnat, MHL Christiaans, PF Vos, HJ Blom, FCGJ Sweep, PNM Demacker, LB Hilbrands 


\section{Abstract}

Cyclosporin is considered to contribute to the high cardiovascular morbidity and mortality in patients after renal transplantation. Tacrolimus may be more favourable in this respect, but controlled data are scarce. In this prospective randomized study in 124 stable renal transplant patients, we investigated the effects of conversion from cyclosporin to tacrolimus on cardiovascular risk factors and renal function. Follow-up was six months. Statistical analysis was performed by ANOVA for repeated measurements. The serum creatinine level decreased from $137 \pm 30 \mu \mathrm{mol} / 1$ to $131 \pm 29 \mu \mathrm{mol} / \mathrm{l}(P<0.01)$. Three months after conversion from cyclosporin to tacrolimus, mean blood pressure significantly decreased from $104 \pm 13$ to $99 \pm 12 \mathrm{mmHg}(P<0.001)$. Serum LDL-cholesterol decreased from $3.48 \pm 0.80$ to $3.11 \pm 0.74 \mathrm{mmol} / 1(P<0.001)$ and serum apolipoprotein $\mathrm{B}$ decreased from $1018 \pm 189$ to $935 \pm 174 \mathrm{mg} / 1(P<0.001)$. Serum triglycerides decreased from $2.11 \pm 1.12$ to $1.72 \pm 0.94 \mathrm{mmol} / 1(P<0.001)$. In addition, both rate and extent of LDL oxidation were reduced. The fibrinogen level decreased from $3638 \pm 857$ to $3417 \pm 751 \mathrm{mg} / \mathrm{l}(P<0.05)$. Plasma homocysteine concentration did not change. Three months after conversion, plasma fasting glucose level temporarily increased from $5.4 \pm 1.3 \mathrm{mmol} / 1$ to $5.8 \pm 1.9 \mathrm{mmol} / \mathrm{l}(P<0.05)$. Conversion to tacrolimus resulted in a significant reduction of the Framingham risk score. In conclusion, conversion from cyclosporin to tacrolimus in stable renal transplant patients has a beneficial effect on renal function, blood pressure, serum concentration and atherogenic properties of serum lipids, and fibrinogen. 


\section{Introduction}

During the past two decades, cyclosporin has proven to be a valuable immunosuppressive drug that has contributed to a significant reduction in the incidence of acute rejection after renal transplantation. However, cyclosporin also increases cardiovascular risk profiles 1 . Ultimately up to $63 \%$ of renal transplant patients die of cardiovascular disease ${ }^{2}$. The increased cardiovascular risk profile due to cyclosporin is ascribed to both a quantitative increase in low-density lipoprotein (LDL)-particles and to an increased oxidizability of the LDLparticles $^{3-5}$. Use of cyclosporin is also associated with increased plasma lipoprotein(a) $(\operatorname{Lp}(a))^{6}$ and homocysteine levels 7 , but these effects are not unequivocal $^{8}$. In addition, unfavourable effects on the fibrinolytic system by cyclosporin have been described 9 . Apart from these disadvantageous effects of cyclosporin on several metabolic cardiovascular risk factors, cyclosporin also leads to an elevation of blood pressure ${ }^{4}$. These side effects not only contribute to the high cardiovascular morbidity in renal transplant patients, but they may also lead to an accelerated loss of graft function ${ }^{10-12}$.

Tacrolimus is like cyclosporin a calcineurin inhibitor with even more potent immunosuppressive properties. The use of tacrolimus after renal transplantation is associated with a less unfavourable effect on blood pressure and serum lipid levels, but evidence from controlled studies is scarce ${ }^{4,13}$. Little is known about the differential effects of cyclosporin and tacrolimus on established cardiovascular risk factors like homocysteine, oxidizability of LDL-particles, and fibrinogen levels. In the present study, we investigated the effect of conversion from cyclosporin to tacrolimus in renal transplant patients on the cardiovascular risk profile and on graft function in a multicenter prospective randomized design.

\section{Material and methods}

\section{Patients}

To be eligible for the study the patients had to be at least one year posttransplantation with a stable endogenous creatinine clearance exceeding $20 \mathrm{ml} / \mathrm{min}$. Immunosuppressive treatment at enrollment had to include cyclosporin with trough levels ranging from 50 to $200 \mathrm{ng} / \mathrm{ml}$, and prednisone, with or without azathioprine or mycophenolate mofetil.

\section{Study protocol}

This prospective randomized open-label study was performed in four renal transplant centers. After stratification for center, patients were randomized in a 
1:1-fashion to either continuation of cyclosporin or conversion to tacrolimus. Total follow-up was six months. Evaluation took place at baseline and after three and six months. Target drug trough levels were 50 to $200 \mathrm{ng} / \mathrm{ml}$ for cyclosporin and 5 to $8 \mathrm{ng} / \mathrm{ml}$ for tacrolimus. Lipid-lowering drug treatment and antihypertensive treatment preferably remained unchanged. Adjustment of lipidlowering medication was allowed when the serum total cholesterol level was below $4.0 \mathrm{mmol} / \mathrm{l}$ or exceeded $6.5 \mathrm{mmol} / 1$, or when the serum triglyceride level exceeded $4.0 \mathrm{mmol} / \mathrm{l}$. Changes in antihypertensive medication were allowed when the systolic blood pressure was $>160 \mathrm{mmHg}$ or $<120 \mathrm{mmHg}$, or when the diastolic blood pressure was $>90 \mathrm{mmHg}$ or $<70 \mathrm{mmHg}$. Blood pressure was measured in supine position manually after five minutes of rest in all participating patients. In patients from three out of four participating centers, blood pressure was also measured using an automated device (Dinamap, Critikon Inc., Tampa, USA) during 30 minutes. The diagnosis of diabetes mellitus was based on the fasting glucose levels, with a cut-off value of $7.0 \mathrm{mmol} / 1$, measured on two separate occasions, in accordance with the American Diabetes Association criteria ${ }^{14}$. Framingham risk scores were calculated using age, smoking behaviour, the presence of diabetes mellitus, fasting LDL- and HDL-cholesterol levels and the systolic and diastolic blood pressure ${ }^{15}$. The study was approved by the medical ethics committee of each participating center. All patients gave written informed consent.

\section{Laboratory procedures}

All blood was drawn after an overnight fast, during morning hours in sitting position. The blood sample for determination of the parameters of fibrinolysis was drawn after releasing the tourniquet to avoid hemolysis.

Serum lipids and lipoproteins (by a combined ultracentrifugation - precipitation method), Lp(a) (by a commercially available radioimmunoassay) and total apolipoprotein B (by immunonephelometry) were determined as described before $^{16}$. The oxidizability of LDL was determined at baseline and after three months of follow-up by registration of the $234 \mathrm{~nm}$ absorbance of dienes produced by copper added to isolated LDL ${ }^{17}$. Both EDTA-plasma samples of each person were stored at $-80^{\circ} \mathrm{C}$ (with $10 \mu \mathrm{l} 600 \mathrm{~g} / \mathrm{l}$ saccharose-solution per ml plasma as cryoprotectant) and analyzed in the same assay. The sample for determination of plasma homocysteine was placed on ice until centrifugation and frozen storage. The plasma homocysteine concentration was measured using a high-performance liquid chromatography procedure, with reverse phase separation and fluorescence detection ${ }^{18}$. Antigen levels of the various components of the plasminogen activator system were determined by ELISA ${ }^{19}$. The cyclosporin level was determined in whole blood with a fluorescence-polarization immune-assay (TDx/TDx FLx cyclosporin monoclonal whole blood assay, Abbott Laboratories, 
Chicago, IL, USA) and the tacrolimus level was determined using a microparticle enzyme immune-assay (IMx Tacrolimus II Assay, Abbott Laboratories).

\section{Statistical analysis}

Statistical analysis was performed using the Statistical Product and Services Solutions (SPSS) package, version 9.0. Values are given as mean \pm standard deviation in case of a normal distribution or otherwise as median and interquartile range. Statistical analysis was performed using one-way ANOVA for repeated measurements. If the ANOVA revealed a statistically significant difference, post hoc comparisons were performed using Bonferroni‘s correction for multiple comparisons.

For parameters that were only determined at two time-points, Student's t-test was used in case of normal distribution and otherwise Wilcoxon signed ranks test was used. For correlations, Pearson's product moment correlation coefficient was used and for analysis of proportions Fisher's exact test was applied. Results are analyzed on an intention-to-treat base for renal function and blood pressure, whereas the metabolic parameters are analyzed in the patients who remained on study medication during follow-up. A $P$-value of less than 0.05 was considered statistically significant.

\section{Results}

Between February 1999 and September 2000, 124 patients were included in the study. Baseline characteristics are shown in Table 7.1. Sixty-four patients were converted to tacrolimus (conversion group) and 60 patients remained on cyclosporin (control group). There were no baseline differences between both groups in serum creatinine level, blood pressure, or any metabolic parameter. Mean baseline cyclosporin trough level was $129 \pm 42 \mathrm{ng} / \mathrm{ml}$ in the control group versus $130 \pm 42 \mathrm{ng} / \mathrm{ml}$ in the conversion group (N.S.). In the control group, the cyclosporin trough level remained unchanged during follow-up. In the conversion group, the mean tacrolimus trough level was $7.4 \pm 1.8 \mathrm{ng} / \mathrm{ml}$ after three months and $7.4 \pm 1.9 \mathrm{ng} / \mathrm{ml}$ after six months of follow-up. Baseline prednisone dose was $0.10 \pm 0.04 \mathrm{mg} / \mathrm{kg}$ in the conversion group and $0.10 \pm 0.04 \mathrm{mg} / \mathrm{kg}$ in the control group, baseline azathioprine dose was $0.88 \pm 0.40 \mathrm{mg} / \mathrm{kg}(\mathrm{n}=10)$ in the conversion group and $0.95 \pm 0.47 \mathrm{mg} / \mathrm{kg}(\mathrm{n}=15)$ in the control group. In the control group, two patients used mycophenolate mofetil $2000 \mathrm{mg} /$ day. The dosages of prednisone, azathioprine and mycophenolate mofetil remained unchanged throughout the study period. 
Table 7.1 Baseline patient demographics.

\begin{tabular}{lcc}
\hline & $\begin{array}{c}\text { Cyclosporin } \\
(\mathbf{n}=\mathbf{6 0})\end{array}$ & $\begin{array}{c}\text { Tacrolimus } \\
\text { (n=64) }\end{array}$ \\
\hline Time after RTX (years) & $6.6 \pm 3.7$ & $5.7 \pm 2.7$ \\
Sex (m/f) & $34 / 26$ & $41 / 23$ \\
Age (years) & $50 \pm 13$ & $50 \pm 14$ \\
Race & & \\
$\quad$ caucasian & $57(95 \%)$ & $60(94 \%)$ \\
black & 0 & 0 \\
other & $3(5 \%)$ & $4(6 \%)$ \\
Original renal disease & & \\
chronic glomerulonephritis & $17(28 \%)$ & $20(31 \%)$ \\
chronic pyelonephritis & $5(8 \%)$ & $11(17 \%)$ \\
polycystic kidney disease & $13(22 \%)$ & $13(20 \%)$ \\
diabetic nephropathy & $3(5 \%)$ & $1(2 \%)$ \\
otherwise & $19(32 \%)$ & $17(27 \%)$ \\
unknown & $3(5 \%)$ & $2(3 \%)$ \\
Diabetes mellitus & & \\
type 1 & $5(8 \%)$ & $2(3 \%)$ \\
type 2 & $3(5 \%)$ & $6(9 \%)$ \\
\hline
\end{tabular}

Mean \pm SD; no significant differences. RTX: renal transplantation.

In the control group, two patients died, one of a cardiac event and one of sepsis. In this group, a further three patients were withdrawn from the study for the following reasons: patient's wish to switch to tacrolimus, patient's wish to stop prednisone and one patient lost to follow-up. In the conversion group, one patient died of a cardiac event. In this group, six patients were withdrawn from the study, due to worsening of pre-existent diabetes mellitus $(n=1)$, new-onset diabetes mellitus $(n=1)$, headache $(n=2)$, allergic skin reaction to tacrolimus $(n=1)$, and progression of pre-existent proteinuria $(n=1)$. A renal graft biopsy in the latter patient, taken four months after conversion to tacrolimus, showed signs of chronic allograft nephropathy.

\section{Renal function and blood pressure}

After conversion from cyclosporin to tacrolimus, no acute rejections occurred. After six months of follow-up, conversion from cyclosporin to tacrolimus resulted in a significant decrease in the serum creatinine level from $137 \pm 30$ to $131 \pm 29$ $\mu \mathrm{mol} / \mathrm{l}$ (Table 7.2). Urinary protein excretion showed a small but significant increment in the cyclosporin group, whereas it remained unchanged in the tacrolimus group.

At three months of follow-up, the manually measured systolic and diastolic blood pressure were significantly decreased in the tacrolimus group from $144 \pm 21 \mathrm{mmHg}$ 
to $138 \pm 18 \mathrm{mmHg}$ and $84 \pm 12 \mathrm{mmHg}$ to $80 \pm 11 \mathrm{mmHg}$, respectively (Table 7.2). These changes were no longer significant after six months of follow-up. These results did not change when only patients that remained on study medication during follow-up were analyzed.

Table 7.2 Renal function and blood pressure.

\begin{tabular}{|c|c|c|c|c|c|c|}
\hline & \multicolumn{3}{|c|}{ Cyclosporin $(n=60)$} & \multicolumn{3}{|c|}{ Tacrolimus $(n=64)$} \\
\hline & baseline & 3 months & 6 months & baseline & 3 months & 6 months \\
\hline Serum creatinine $(\mu \mathrm{mol} / \mathrm{l})$ & $143 \pm 48$ & $147 \pm 48$ & $146 \pm 53$ & $137 \pm 30$ & $136 \pm 32$ & $131 \pm 29 \mathrm{~b}$ \\
\hline \multirow[t]{2}{*}{ Proteinuria (g/24 hrs) } & 0.20 & & $0.22 \mathrm{~b}$ & 0.20 & & 0.14 \\
\hline & $(0.10-0.52)$ & & $(0.11-0.70)$ & $(0.08-0.50)$ & & $(0.08-0.44)$ \\
\hline Body Weight (kg) & $75.0 \pm 14.0$ & & $74.8 \pm 14.3$ & $77.8 \pm 15.8$ & & $76.5 \pm 15.1^{\mathrm{c}}$ \\
\hline \multicolumn{7}{|l|}{ Blood pressure manually } \\
\hline mean $(\mathrm{mmHg})$ & $102 \pm 10$ & $104 \pm 11$ & $104 \pm 11$ & $104 \pm 13$ & $99 \pm 12^{c}$ & $101 \pm 13$ \\
\hline systolic $(\mathrm{mmHg})$ & $140 \pm 15$ & $144 \pm 18$ & $142 \pm 18$ & $144 \pm 21$ & $138 \pm 18^{b}$ & $140 \pm 21$ \\
\hline diastolic $(\mathrm{mmHg})$ & $83 \pm 9$ & $84 \pm 0$ & $85 \pm 10$ & $84 \pm 12$ & $80 \pm 11^{c}$ & $82 \pm 10$ \\
\hline \multicolumn{7}{|l|}{ Blood pressure dynamap } \\
\hline mean $(\mathrm{mmHg})$ & $105 \pm 13$ & $105 \pm 11$ & $106 \pm 10$ & $106 \pm 14$ & $101 \pm 9$ & $102 \pm 11$ \\
\hline systolic $(\mathrm{mmHg})$ & $141 \pm 17$ & $142 \pm 14$ & $144 \pm 14$ & $141 \pm 19$ & $135 \pm 15$ & $137 \pm 18$ \\
\hline diastolic $(\mathrm{mmHg})$ & $83 \pm 9$ & $82 \pm 9$ & $83 \pm 9$ & $82 \pm 10$ & $79 \pm 8$ & $79 \pm 9$ \\
\hline Number of antihypertensive drugs & $1.9 \pm 1.0$ & $1.9 \pm 1.0$ & $2.0 \pm 1.0$ & $2.1 \pm 1.1$ & $2.0 \pm 1.1$ & $2.2 \pm 1.1$ \\
\hline
\end{tabular}

Mean \pm SD or median and interquartile range; ${ }^{a}=P<0.05 ; \mathrm{b}=P<0.01 ; \mathrm{c}=P<0.001$ for comparison with baseline. Dinamap blood pressure was determined in 84 patients from three participating centers

\section{Lipid profile}

After conversion from cyclosporin to tacrolimus, serum total and LDLcholesterol, triglycerides, and apolipoprotein B levels decreased, whereas HDLcholesterol and $\mathrm{Lp}$ (a) level did not change (Table 7.3). Entering the use of a statin as a covariate, revealed a significant influence of statin-usage on the change in LDL-cholesterol level after conversion. In the tacrolimus group, patients on statins had a lower baseline LDL-cholesterol level compared to patients not using statins $(3.20 \pm 0.60 \mathrm{mmol} / 1$ versus $3.86 \pm 0.90 \mathrm{mmol} / 1, P<0.01)$, and there was no significant reduction in LDL-cholesterol level six months after conversion to tacrolimus in patients using statins $(0.22 \pm 0.66 \mathrm{mmol} / \mathrm{l}, P=0.31$ for comparison with baseline), compared to a significant reduction in patients not using statins $(0.65 \pm 0.64 \mathrm{mmol} / 1, P<0.001$ for comparison with baseline). 
Table 7.3 Parameters of lipid metabolism.

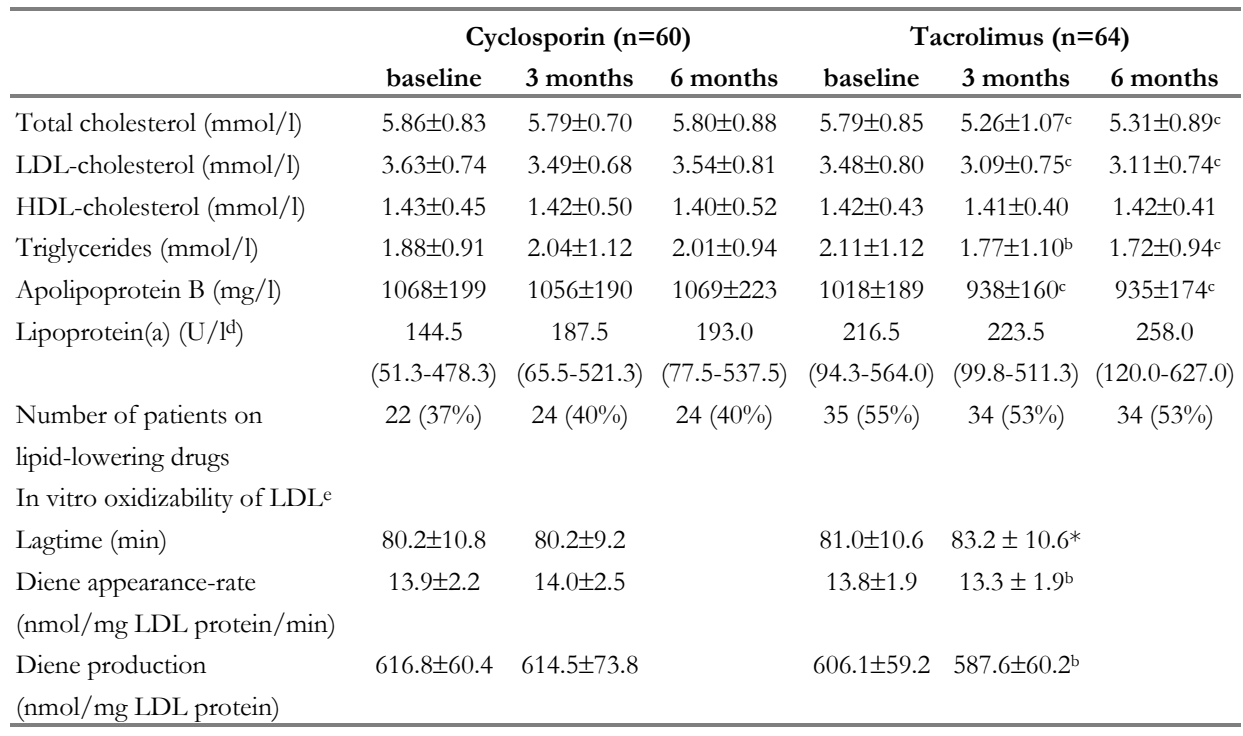

Mean \pm SD or median and interquartile range, ${ }^{a}=P<0.05 ; \mathrm{b}=P<0.01 ; \mathrm{c}=P<0.001$ for comparison with baseline. ${ }^{\mathrm{d}=}$ conversion-factor: $1 \mathrm{U} / \mathrm{l}$ is about $0.7 \mathrm{mg} / \mathrm{l}$ (phenotype-dependent). ${ }^{\mathrm{e}=}$ determined in two participating centers ( 41 cyclosporin patients and 42 tacrolimus patients).

At three months of follow-up, tacrolimus treated patients showed a significant increase in the resistance to in vitro oxidation of the LDL-particles, as shown by an increment in the lagtime for oxidation and decrements in the appearance-rate of dienes and the total amount of diene-formation. The reduction in oxidizability of the LDL-particle was not dependent on the concomitant use of statins.

In two cyclosporin treated patients, statins were started and in one patient the dosage was increased. In the tacrolimus group, the statin was stopped in one patient and the dosage was reduced in another patient.

\section{Glucose regulation}

In the tacrolimus group, the plasma fasting glucose level showed an increase after three months of follow-up. After six months of follow-up the glucose level was not different from the baseline value (Table 7.4). When analyzing diabetic and non-diabetic patients separately, there were no significant changes in glucose level in either group during follow-up. $\mathrm{HbA}_{1} \mathrm{c}$ levels were determined in one center $(n=35)$ and there were no significant changes after conversion to tacrolimus. When analysing diabetic and non-diabetic patients separately, again there were no significant changes in $\mathrm{HbA}_{1} \mathrm{c}$ level in either group after conversion to tacrolimus. 
Six months after conversion from cyclosporin to tacrolimus, four patients $(6 \%)$ had developed new-onset diabetes mellitus, versus two patients in the control group $(3 \%$, N.S.). In the conversion group, the new-onset diabetes mellitus could be treated with oral antidiabetic drugs in two cases and in the other two patients only dietary measures were necessary, resulting in plasma fasting glucose levels persistently below $6 \mathrm{mmol} / \mathrm{l}$ and an $\mathrm{HbA}_{1} \mathrm{c}$ below $6.1 \%$. Both patients that were put on antidiabetic medication wanted to be taken off the tacrolimus and they were converted from tacrolimus to azathioprine. No acute rejections occurred. In one patient the oral antidiabetic medication could be stopped four months after withdrawal of tacrolimus, in the second patient the oral antidiabetic medication was continued in a low dosage. In three out of six tacrolimus-treated patients with pre-existent type 2 diabetes mellitus, the dose of the oral antidiabetic medication had to be increased, versus none out of three patients in the control group. $\mathrm{HbA}_{1} \mathrm{c}$ level did not change in either group during follow-up.

Table 7.4 Glucose, parameters of fibrinolysis and plasma homocysteine.

\begin{tabular}{lcccccc}
\hline & \multicolumn{3}{c}{ Cyclosporin (n=60) } & \multicolumn{3}{c}{ Tacrolimus (n=64) } \\
& baseline & $\mathbf{3}$ months & $\mathbf{6}$ months & baseline & $\mathbf{3}$ months & $\mathbf{6}$ months \\
\hline Glucose (mmol/l) & $5.5 \pm 1.5$ & $5.3 \pm 1.2$ & $5.2 \pm 1.1$ & $5.4 \pm 1.3$ & $5.8 \pm 1.9^{\mathrm{a}}$ & $5.6 \pm 1.8$ \\
$\quad$ non-diabetic & $5.1 \pm 0.6$ & $5.0 \pm 0.8$ & $5.1 \pm 0.7$ & $5.1 \pm 0.9$ & $5.4 \pm 0.8$ & $5.3 \pm 1.0$ \\
$\quad$ diabetic & $8.6 \pm 2.9$ & $7.4 \pm 2.1$ & $6.4 \pm 2.5$ & $8.0 \pm 1.9$ & $9.2 \pm 4.4$ & $8.9 \pm 3.4$ \\
HbA1c $(\%)$ & $5.6 \pm 0.5$ & $5.6 \pm 0.5$ & $5.6 \pm 0.4$ & $5.8 \pm 0.9$ & $6.1 \pm 1.7$ & $6.1 \pm 1.4$ \\
$\quad$ non-diabeticd & $5.5 \pm 0.4$ & $5.6 \pm 0.4$ & $5.5 \pm 0.3$ & $5.6 \pm 0.4$ & $5.5 \pm 0.5$ & $5.6 \pm 0.5$ \\
$\quad$ diabetic & $7.9 \pm 0.6$ & $7.6 \pm 0.6$ & $7.6 \pm 0.7$ & $8.8 \pm 1.7$ & $9.2 \pm 1.2$ & $8.5 \pm 1.0$ \\
Fibrinogen $(\mathrm{mg} / \mathrm{l})$ & $3543 \pm 679$ & $3569 \pm 681$ & $3637 \pm 708$ & $3638 \pm 857$ & $3367 \pm 763 \mathrm{c}$ & $3417 \pm 751^{\mathrm{a}}$ \\
tPA (ng/ml) & $3.80 \pm 15.68$ & $3.29 \pm 1.42$ & $3.22 \pm 1.37$ & $4.07 \pm 2.71$ & $3.65 \pm 1.78$ & $3.68 \pm 2.04$ \\
PAI-I $(\mathrm{ng} / \mathrm{ml})$ & $26.82 \pm 15.68$ & $33.23 \pm 20.78$ & $34.95 \pm 19.13$ & $30.04 \pm 20.57$ & $30.38 \pm 17.68$ & $31.65 \pm 18.29$ \\
tPA-PAI complex $(\mathrm{ng} / \mathrm{ml})$ & $8.38 \pm 4.77$ & $8.20 \pm 4.03$ & $7.77 \pm 4.56$ & $10.19 \pm 7.42$ & $9.53 \pm 6.37$ & $9.79 \pm 6.57$ \\
uPA $(\mathrm{ng} / \mathrm{ml})$ & $1.22 \pm 0.37$ & $1.27 \pm 0.41$ & $1.23 \pm 0.40$ & $1.26 \pm 0.35$ & $1.17 \pm 0.37 \mathrm{a}$ & $1.16 \pm 0.33^{\mathrm{a}}$ \\
Homocysteine $(\mu \mathrm{mol} / \mathrm{l})$ & 18.6 & 19.0 & 18.1 & 18.4 & 18.0 & 16.6 \\
& $(16.3-22.1)$ & $(14.6-21.6)$ & $(14.5-21.2)$ & $(14.7-23.7)$ & $(13.9-24.7)$ & $(14.6-21.1)$ \\
\hline
\end{tabular}

Mean \pm SD or median and interquartile range; ${ }^{a}=P<0.05, b=P<0.01, c=P<0.001$ for comparison with baseline. $\mathrm{d}=$ determined in all patients from one center $(17$ cyclosporin patients and 18 tacrolimus patients).

\section{Other metabolic cardiovascular risk factors}

Baseline homocysteine level correlated significantly with baseline serum creatinine value $(\mathrm{r}=0.43, P<0.001)$. During follow-up, there were neither changes in plasma homocysteine levels (Table 7.4) nor in serum folic acid concentration (data not shown). 
After conversion from cyclosporin to tacrolimus, the plasma fibrinogen level and the plasma urokinase type plasminogen activator (uPA) level decreased. There were no changes in the plasma levels of plasminogen activator inhibitor type I (PAI-I), tissue-type plasminogen (tPA) and tPA-PAI-complex (Table 7.4).

\section{Framingham risk score}

After conversion to tacrolimus a significant reduction of the Framingham risk score was observed at three months of follow-up, but no longer at six months after conversion $(P=0.13)$. Entering the use of a statin as co-variate, revealed a significant influence of statin-usage on the change in the Framingham risk score after conversion. In the patients not using a statin, the framingham risk score significantly decreased from $4.0 \pm 6.1$ to $2.6 \pm 6.3$ at six months $(P<0.001)$, whereas in patients using a statin the framingham risk score did not change (6.0 44.1 at baseline and 6.1 \pm 4.6 at six months, Figure 7.1.).
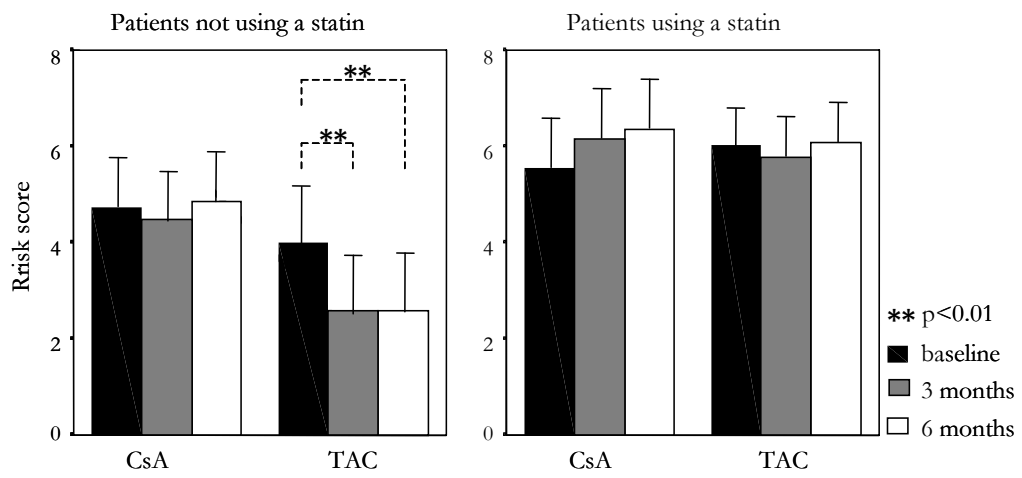

Figure 7.1 Framingham risk score (mean and SEM) on cyclosporin (CsA) versus tacrolimus (TAC).

\section{Discussion}

The grossly enlarged cardiovascular risk in renal transplant patients is related to a combination of partly related risk factors, such as hypertension, hyperlipidemia, hereditary risk, diabetes mellitus, physical inactivity, obesity, hyperuricemia, hyperhomocysteinemia and hyperparathyroidism. In order to address the pressing challenge in renal transplantation of improving patient survival and long-term graft function, this broad spectrum of risk factors has to be optimized, without putting the graft function at risk. In this prospective randomized controlled trial, conversion from cyclosporin to tacrolimus in stable renal transplant patients 
resulted in an improvement in a number of cardiovascular risk factors, together with an improvement in renal function without risking acute rejection.

After conversion to tacrolimus the serum LDL-cholesterol level is reduced and the LDL-particles are less susceptible to oxidation. Cyclosporin increases serum LDL-cholesterol level by inhibiting the synthesis of LDL-receptors in the liver, thereby interfering with the LDL-receptor mediated catabolism in the liver ${ }^{20}$. The improvement in serum LDL-cholesterol level after conversion to tacrolimus might be due to the withdrawal of this inhibition of the LDL-receptor-production. However, this quantitative improvement of LDL-cholesterol is only achieved in patients not treated with statins. In patients using statins, baseline LDLcholesterol already is reduced and no further reduction is observed after conversion from cyclosporin to tacrolimus, at least not in this subgroup with a limited number of patients. The atherogenicity of LDL-cholesterol not only depends on its serum concentration, but also on the oxidizability of the particles ${ }^{21}$. Therefore, it is important that conversion from cyclosporin to tacrolimus not only reduced the serum concentration of LDL-cholesterol, but also the oxidizability of the LDL-particle. Both the increment in the lag time before initiation of oxidation of LDL and the lower rate of oxidation reflect increased resistance of LDL against oxidation. This reduction in oxidizability occurred both in patients with and without the use of statins, and therefore, conversion to tacrolimus was beneficial for both patient groups. The reduced oxidizability of the LDL-particles is likely to be associated with the concurrent decrease in serum triglycerides after conversion to tacrolimus. Reduced levels of serum triglycerides and apolipoprotein B are associated with an altered, less dense composition of the LDL-particles ${ }^{22}$. These lighter LDL-particles contain more lipids compared to the protein component, resulting in conformational changes with a diminished access for free radicals and pro-oxidants such as copper to cause oxidation of the fatty acids ${ }^{23}$. Indeed, the lipid-enriched lower-density LDL-subfractions have been shown to be more resistant against oxidation ${ }^{24}$. Furthermore, lighter LDL-particles are more easily cleared from the circulation by the high-affinity LDL-receptor, leading to a shorter plasma residence time, during which the particle is susceptible to in-vivo oxidation $^{25}$. In line with our findings, an increased lag time for oxidation in patients on tacrolimus compared to patients on cyclosporin was recently reported $^{26}$. In contrast, Varghese et al. reported that LDL from tacrolimus-treated patients was more susceptible to oxidation compared to cyclosporin-treated patients ${ }^{27}$. However, in that cross-sectional study, the blood level of cyclosporin was very low, averaging $87 \pm 35 \mathrm{ng} / \mathrm{ml}$, which is below the limit of $120 \mathrm{ng} / \mathrm{ml}$ above which accelerated oxidation of isolated LDL was found 6 .

We previously showed that tacrolimus trough level reduction from 9.5 to $6.4 \mathrm{ng} / \mathrm{ml}$ did not result in a reduction of total and LDL-cholesterol and triglycerides ${ }^{28}$, which makes it unlikely that the improvement in the serum lipid profile in our conversion group is related to a relatively low level of tacrolimus in 
our patients. Also, it is unlikely that the improvement in renal function in the conversion group can account for the improvement in the lipid profile, since the lipid profile was already improved at three months whereas renal function was only improved after six months. Also, there was no correlation between the reduction in LDL-cholesterol or in triglyceride levels and the serum creatinine level after conversion to tacrolimus (data not shown).

Diabetes mellitus is a major cardiovascular risk factor and is also associated with an increased risk of developing graft failure 29 . Although tacrolimus has been reported to be diabetogenic ${ }^{30,31}$, this risk is predominantly present in the initial period after transplantation ${ }^{32}$. After lowering the dose of tacrolimus and withdrawal of steroids, most patients can be weaned off the diabetic medication ${ }^{33}$. In this study, performed in a population that predominantly consisted of Caucasian patients, only a small and temporary increase in fasting glucose level was present at three months after conversion. When analysing diabetic and nondiabetic patients separately, there was no significant change in glucose level in either group during follow-up. This could however be related to reduced power to detect a difference due to a smaller population. We know from previous studies that tacrolimus influences glucose metabolism by reducing pancreatic insulin secretion in a dose-dependent way ${ }^{28}$. Initially, also an increase in insulin resistance has been reported $^{32}$, but this is the result of the co-administration of steroids ${ }^{28}$. At standard maintenance trough levels, no difference between cyclosporin and tacrolimus could be determined with regard to their diabetogenic properties ${ }^{34}$. Therefore, in the majority of patients, conversion from cyclosporin to tacrolimus is safe with regard to the development of diabetes mellitus. The risk of developing diabetes mellitus after conversion to tacrolimus is restricted to patients who already experience a disturbed glucose tolerance prior to therapy, and might be increased in black and hispanic patients who have a greater predisposition to develop post-transplant diabetes $30,32,35$.

In $77 \%$ of our patients, the fasting homocysteine level exceeded the advised upper level of $15 \mu \mathrm{mol} / 1$. An impaired renal function is supposed to be an important factor contributing to hyperhomocysteinemia after renal transplantation ${ }^{7}$ and there are conflicting data on the role of cyclosporin ${ }^{7,8}$. Conversion from cyclosporin to tacrolimus did not result in a significant reduction of the fasting homocysteine level.

The observed reduction in fibrinogen levels after conversion to tacrolimus might also be important in reducing cardiovascular disease, since a high fibrinogen level is an important and independent cardiovascular risk factor ${ }^{36}$. Also, in a crosssectional study, high fibrinogen levels have been associated with chronic renal allograft dysfunction ${ }^{37}$. With regard to determinants of the fibrinolytic system, a reduction in the level of the plasma uPA level was observed in the tacrolimus group. The plasma levels of the other determinants of the fibrinolytic system remained unchanged after conversion. UPA is not only involved in fibrinolysis, 
but also in tissue remodeling during wound healing. High levels of uPA are associated with a higher degree of restenosis after coronary artery angioplasty ${ }^{38}$. There are however no data indicating that reduction of the uPA level has a role in the primary prevention of cardiovascular disease.

The manually measured systolic and diastolic blood pressure were decreased at three months after conversion from cyclosporin to tacrolimus by $7 \pm 18 \mathrm{mmHg}$ and $5 \pm 10 \mathrm{mmHg}$, respectively. In agreement with these findings, a recent study in healthy volunteers showed that, in contrast to cyclosporin, tacrolimus did not increase blood pressure ${ }^{39}$. However, in the current study, the decline in blood pressure tended to wane with time and was no longer significant at six months of follow-up. Measurement of blood pressure with an automated device showed a similar trend, although the differences did not reach statistical significance, probably due to the smaller number of patients in whom the blood pressure was determined automatically. No change in antihypertensive medication or in tacrolimus trough level that could account for the diminished effect of conversion to tacrolimus on blood pressure after six months occurred.

The Framingham risk score was used in order to integrate the differential effects of cyclosporin and tacrolimus on several important components of the cardiovascular risk profile, namely serum lipids, blood pressure, and diabetes mellitus. Conversion to tacrolimus resulted in a significant reduction of the Framingham risk score. This reduction however was only apparent in patients not using statins, indicating that reduction of the LDL-cholesterol level is the main factor influencing the risk score after conversion to tacrolimus. The Framingham risk score was developed in an American population not suffering from specific underlying diseases, and has been validated in several other geographic and ethnic populations ${ }^{40}$. Probably, renal transplant patients will benefit from a reduction of the Framingham risk score. However, the risk score has never been validated in patients suffering from renal disease and, since renal patients experience other contributing pathogenic mechanisms, e.g. hyperparathyroidism and secondary hyperlipidemia, characterized by hypertriglyceridemia and small dense LDLparticles, it is unlikely that the prediction of the absolute risk of coronary heart disease from the Framingham risk score can directly be applied to these patients. Another drawback of this risk score is that it does not take into account several more recently recognized cardiovascular risk factors, like the oxidizability of the LDL-particles and the fibrinogen level.

The improvements in the cardiovascular risk profile as described above not only are important for the cardiovascular morbidity and mortality, but also are likely to be of benefit for long-term graft function. Chronic allograft nephropathy (CAN) is the main cause of late graft loss in renal transplantation. The pathogenesis of CAN is multifactorial. The initiating factors are probably mainly immunologic, while the perpetuating factors are considered to be largely non-immunologic, 
including hyperlipidemia and hypertension ${ }^{41}$. Accordingly, increased levels of LDL-cholesterol and triglycerides are associated with the occurrence of CAN after renal transplantation ${ }^{11,42}$. Oxidatively modified LDL-cholesterol is a chemotactic factor for monocytes and macrophages, both in vascular endothelium and in renal glomeruli and it may cause activation of endothelial cells, smooth muscle cells, mesangial cells, and macrophages ${ }^{43-45}$. Bosmans et al. ${ }^{10}$ showed deposition of LDL and oxidized LDL in a mesangiocapillary way in the glomeruli, in endothelial cells, and in the interstitial space. The amount of oxidized LDL immunostaining was related to the increase in the density of macrophages in the tubulo-interstitial compartment and to the extent of interstitial fibrosis ${ }^{10}$. Therefore, the reduction of LDL-cholesterol and the improvement of LDL-oxidation by tacrolimus may be of benefit for long-term graft survival.

Some studies report that tacrolimus as part of the initial maintenance immunosuppressive therapy leads to a significantly improved long-term renal graft survival compared to cyclosporin, but this effect of tacrolimus is not unequivocal ${ }^{31,46}$. In the analysis of Hariharan et al., tacrolimus was not a significant factor contributing to the recently observed improvements in graft half-life ${ }^{47}$. Possible mechanisms by which tacrolimus could induce an improvement in graft survival include stronger immunosuppression leading to a reduction in the incidence and severity of acute rejections ${ }^{30,31}$, differential effects on renal hemodynamics leading to diminished renal vasoconstriction on tacrolimus ${ }^{39}$, a reduction in blood pressure and serum lipid levels ${ }^{4}$ and a reduction in interstitial fibrosis inducing cytokines like TGF- $\beta^{48}$. Based on this knowledge, it is of interest to see that conversion from cyclosporin to tacrolimus in a later stage after renal transplantation still can lead to an improvement in renal function. In view of the improvement in renal function, the question arises whether equipotent dosages of cyclosporin and tacrolimus have been used. In the participating centers, where mainly patients from Caucasian origin are treated, the standard target tacrolimus trough level after one year post-transplantation ranges from 5 to $8 \mathrm{ng} / \mathrm{ml}^{49}$. Our mean tacrolimus trough level was lower than the level that was achieved in several previously performed long term studies, ranging from $8.5 \mathrm{ng} / \mathrm{ml}$ to approximately $12 \mathrm{ng} / \mathrm{ml}$, but also our cyclosporin level was low compared to these studies, where levels ranging from $174 \mathrm{ng} / \mathrm{ml}$ to approximately $180 \mathrm{ng} / \mathrm{ml}$ were achieved ${ }^{30,50}$. Our tacrolimus and cyclosporin trough levels were comparable with the levels in the study of Mayer et al., who achieved mean trough levels of $7.3 \mathrm{ng} / \mathrm{ml}$ and 119 $\mathrm{ng} / \mathrm{ml}$ for tacrolimus and cyclosporin, respectively ${ }^{31}$. In our population, the maintenance tacrolimus dose was sufficient to prevent acute rejections in all patients. Longer follow-up of our patients will disclose whether the improvement in graft function is sustained.

In conclusion, this study demonstrates that conversion from cyclosporin to tacrolimus results in an improved cardiovascular risk profile, with regard to serum levels as well as atherogenic properties of the lipid profile, the serum fibrinogen 
level and blood pressure. Furthermore, renal function improves. These findings are likely to be of importance in reducing the incidence of cardiovascular disease and may benefit graft survival. The diabetogenic effect of tacrolimus was of clinical relevance in a minor subset of patients, particularly those that already had an impaired glucose tolerance prior to conversion to tacrolimus. Overall, when long-term treatment with calcineurin inhibitors is required, tacrolimus is preferable to cyclosporin for optimization of the cardiovascular risk profile and graft function, except perhaps in patients known to have an impaired glucose tolerance. 


\section{References}

1. Woo YM, Jardine AG, Clark AF, MacGregor MS, Bowman AW, Macpherson SG, Briggs JD, Junor BJ, McMillan MA and Rodger RS. Early graft function and patient survival following cadaveric renal transplantation. Kidney Int 1999;55:692-9.

2. Lindholm A, Albrechtsen D, Frödin L, Tufveson G, Persson NH and Lundgren G. Ischemic heart disease - Major cause of death and graft loss after renal transplantation in Scandinavia. Transplantation 1995;60:451-7.

3. Quaschning T, Mainka T, Nauck M, Rump LC, Wanner C and Krämer-Guth A. Immunosuppression enhances atherogenicity of lipid profile after transplantation. Kidney Int 1999;56(S71):S235-7.

4. Ligtenberg G, Hené RJ, Blankestijn PJ and Koomans HA. Cardiovascular risk factors in renal transplant patients: Cyclosporin A versus tacrolimus. J Am Soc Nephrol 2001;12:368-73.

5. Apanay DC, Neylan JF, Ragab MS and Sgoutas DS. Cyclosporin increases the oxidizability of low-density lipoproteins in renal transplant recipients. Transplantation 1994;58:663-9.

6. Brown JH, Murphy BG, Douglas AF, Short CD, Bhatnagar D, Mackness MI, Hunt LP, Doherty CC and Durrington PN. Influence of immunosuppressive therapy on lipoprotein(a) and other lipoproteins following renal transplantation. Nephron 1997;75:277-82.

7. Arnadottir M, Hultberg B, Vladov V, Nilsson-Ehle P and Thysell H. Hyperhomocysteinemia in cyclosporine-treated renal transplant recipients. Transplantation 1996;61:509-12.

8. Bostom AG, Gohh RY, Beaulieu AJ, Han H, Jacques PF, Selhub J, Dworkin L and Rosenberg IH. Determinants of fasting plasma total homocysteine levels among chronic stable renal transplant recipients. Transplantation 1999;68:257-61.

9. Dorpel MA van den, Man in 't Veld AJ, Levi M, Cate JW ten and Weimar W. Beneficial effects of conversion from cyclosporine to azathioprine on fibrinolysis in renal transplant recipients. Arterioscler Thromb Vasc Biol 1999;19:1555-8.

10. Bosmans JL, Holvoet P, Dauwe SE, Ysebaert DK, Chapelle T, Jurgens A, Kovacic V, Marck EA van, Broe ME de and Verpooten GA. Oxidative modification of low-density lipoproteins and the outcome of renal allografts at 1 1/2 years. Kidney Int 2001;59:2346-56.

11. Dimény E, Wahlberg J, Lithell $\mathrm{H}$ and Fellström B. Hyperlipidaemia in renal transplantation risk factor for long-term graft outcome. Eur J Clin Invest 1995;25:574-83.

12. Peschke B, Scheuermann, Geiger H, Bölscher S, Kachel H-G and Lenz T. Hypertension is associated with hyperlipidemia, coronary heart disease and chronic graft failure in kidney transplant recipients. Clin Nephrol 1999;51:290-5.

13. McCune TR, Thacker LR II, Peters TG, Mulloy L, Rohr MS, Adams PA, Yium J, Light JA, Pruett T, Gaber AO, Selman SH, Jonsson J, Hayes JM, Wright FH jr, Armata T, Blanton J and Burdick JF. Effects of tacrolimus on hyperlipidemia after successful renal transplantation. A Southeastern Organ Procurement Foundation Multicenter Clinical Study. Transplantation 1998;65:87-92.

14. Expert Committee on the Diagnosis and Classification of Diabetes Mellitus. American Diabetes Association: clinical practice recommendations 2002. Diabetes Care 2002;25(S1): S1-147. 
15. Wilson PWF, D'Agostino RB, Levy D, Belanger AM, Silbershatz H and Kannel WB. Prediction of coronary heart disease using risk factor categories. Circulation 1998;97:1837-47.

16. Demacker PN, Veerkamp MJ, Bredie SJ, Marcovina SM, Graaf J de and Stalenhoef AF. Comparison of the measurement of lipids and lipoproteins versus assay of apolipoprotein $\mathrm{B}$ for estimation of coronary heart disease risk: A study in familial combined hyperlipidemia. Atherosclerosis 2000;153:483-90.

17. Kleinveld HA, Hak-Lemmers HL, Stalenhoef AF and Demacker PN. Improved measurement of low-density-lipoprotein susceptibility to copper-induced oxidation: Application of a short procedure for isolating low-density lipoprotein. Clin Chem 1992;38:2066-72.

18. te-Poele-Pothoff MT, Berg M van den, Franken DG, Boers GH, Jakobs C, Kroon IF de, Eskes TK, Trijbels JM and Blom HJ. Three different methods for the determination of total homocysteine in plasma. Ann Clin Biochem 1995;32:218-20.

19. Grebenschikov N, Geurts-Moespot A, Witte H de, Heuvel J, Leake R, Sweep E and Benraad T. A sensitive and robust assay for urokinase and tissue-type plasminogen activators (uPA and tPA) and their inhibitor type 1 (PAI-1) in breast tumor cytosols. Int J Biol Markers 1997;12:614.

20. Rayyes OA, Wallmark A and Florén C-H. Cyclosporine inhibits catabolism of low-density lipoproteins in HepG2 cells by about $25 \%$. Hepatology 1996;24:613-9.

21. Steinberg D, Parthasarathy S, Carew TE, Khoo JC and Witztum JL. Beyond cholesterol. Modifications of low density lipoprotein that increase its atherogenicity. N Engl J Med 1989; 320:915-24.

22. McKeone BJ, Patsch JR and Pownall HJ. Plasma triglycerides determine low density lipoprotein composition, physical properties, and cell-specific binding in cultured cells. J Clin Invest 1993;91:1926-33.

23. Aviram M, Lund-Katz S, Philips MC and Chait A. The influence of the triglyceride content of low density lipoprotein on the interaction of apolipoprotein B-100 with cells. J Biol Chem 1988;263:16842-8.

24. Graaf J de, Hak-Lemmers HL, Hectors MP, Demacker PN, Hendriks JC and Stalenhoef AF. Enhanced susceptibility to in vitro oxidation of the dense low density lipoprotein subfraction in healthy subjects. Arterioscler Thromb 1991;11:298-306.

25. Teng B, Sniderman AD, Soutar AK and Thompson GR. Metabolic basis of hyperapobetalipoproteinemia. Turnover of apolipoprotein B in low density lipoprotein and its precursors and subfractions compared with normal and familial hypercholesterolemia. J Clin Invest 1986;77:663-72.

26. Venkiteswaran K, Sgoutas DS, Santanam N and Neylan JF. Tacrolimus, cyclosporine and plasma lipoproteins in renal transplant recipients. Transpl Int 2001;14:405-10.

27. Varghese Z, Fernando R, Turakhia G, Psimenou E, Brunton C, Fernando ON, Davenport A, Burns A, Sweny P, Powis SH and Moorhead JF. Oxidizability of low-density lipoproteins from neoral and tacrolimus-treated renal transplant patients. Transplant Proc 1998;30:2043-6.

28. Boots JMM, Duijnhoven EM van, Christiaans MHL, Wolffenbuttel BHR and Hooff JP van. Glucose metabolism in renal transplant recipients on tacrolimus: the effect of steroid withdrawal and tacrolimus trough level reduction. J Am Soc Nephrol 2002;13:221-7.

29. Miles AMV, Sumrani N, Horowitz R, Homel P, Maursky V, Markell MS, Distant DA, Hong $\mathrm{JH}$, Sommer BG and Friedman EA. Diabetes mellitus after renal transplantation. As deleterious as non-transplant-associated diabetes? Transplantation 1998;65:380-4. 
30. Pirsch JD, Miller J, Deierhoi MH, Vincenti F and Filo RS for the FK506 Kidney Transplant Study Group. A comparison of tacrolimus (FK506) and cyclosporine for immunosuppression after cadaveric renal transplantation. Transplantation 1997;63:977-83.

31. Mayer AD for the European Tacrolimus Multicentre Renal Study Group. Chronic rejection and graft half-life: Five year follow-up of the European Tacrolimus Multicenter Renal Study. Transplant Proc 2002;34:1491-2.

32. Weir MR and Fink JC. Risk for posttransplant diabetes mellitus with current immunosuppressive medications. Am J Kidney Dis 1999;34:1-13.

33. Boots JMM, Duijnhoven EM, Christiaans MHL, Nieman FHM, Suylen RJ van and Hooff JP van. Single center experience with tacrolimus versus cyclosporin-Neoral in renal transplant recipients. Transpl Int 2001;14:370-83.

34. Duijnhoven EM van, Christiaans MHL, Boots JMM, Nieman FHR, Wolffenbuttel BHR and Hooff JP van. Glucose metabolism in the first 3 years after renal transplantation in patients on tacrolimus versus cyclosporine-based immunosuppression. J Am Soc Nephrol 2002;13:213-20.

35. Duijnhoven EM van, Boots JMM, Christiaans MHL, Wolffenbuttel BHR and Hooff JP van. Influence of tacrolimus on glucose metabolism before and after renal transplantation: a prospective study. J Am Soc Nephrol 2001;12:583-8.

36. Kannel WB, Wolf PA, Castelli WP and D'Agostino RB. Fibrinogen and risk of cardiovascular disease. The Framingham study. J Am Med Assoc 1987;258:1183-6.

37. Fernández-Miranda C, Morales JM, Porres A, Gomez-Gerique J, Guijarro C, Aranda JL, Andres A and Rodicio JLDPA. Increased lipoproteins and fibrinogen in chronic renal allograft dysfunction. Am J Nephrol 1997;17:445-9.

38. Strauss BH, Lau HK, Bowman KA, Sparkes J, Chisholm RJ, Garvey MB, Fenkell LL, Natarajan MK, Singh I and Teitel JM. Plasma urokinase antigen and plasminogen activator inhibitor-1 antigen levels predict angiographic coronary restenosis. Circulation 1999;100: 1616-22.

39. Klein IH, Abrahams A, Ede T van, Hené RJ, Koomans HA and Ligtenberg G. Different effects of tacrolimus and cyclosporine on renal hemodynamics and blood pressure in healthy subjects. Transplantation 2002;73:732-6.

40. Grundy SM, Balady GJ, Criqui MH, Fletcher G, Greenland P, Hiratzka LF, Houston-Miller N, Kris-Etherton P, Krumholz HM, LaRosa J, Ockene IS, Pearson TA, Reed J, Washington R and Smith SC jr. Primary prevention of coronary heart disease: guidance from Framingham: A statement for healthcare professionals from the AHA Task Force on Risk Reduction. American Heart Association. Circulation 1998;97:1876-87.

41. Massy ZA, Guijarro C, Wiederkehr MR, Ma JZ and Kasiske BL. Chronic renal allograft rejection: Immunologic and nonimmunologic risk factors. Kidney Int 1996;49:518-24.

42. Isoniemi H, Nurminen M, Tikkanen MJ, Willebrand E von, Krogerus L, Ahonen J, Eklund B, Hockerstedt K, Salmela K and Hayry P. Risk factors predicting chronic rejection of renal allografts. Transplantation 1994;57:68-72.

43. Pai R, Kirschenbaum MA and Kamanna VS. Low-density lipoprotein stimulates the expression of macrophage colony-stimulating factor in glomerular mesangial cells. Kidney Int 1995;48: 1254-62.

44. Kamanna VS, Pai R, Ha H, Kirschenbaum MA and Roh DD. Oxidized low-density lipoprotein stimulates monocyte adhesion to glomerular endothelial cells. Kidney Int 1999;55: 2192-202. 
45. Quinn MT, Parthasarathy S, Fong LG and Steinberg D. Oxidatively modified low density lipoproteins: a potential role in recruitment and retention of monocytes/macrophages during atherogenesis. Proc Natl Acad Sci U S A 1987;84:2995-8.

46. Vincenti F, Jensik SC, Filo RS, Miller J and Pirsch J. A long-term comparison of tacrolimus (FK506) and cyclosporine in kidney transplantation: evidence for improved allograft survival at five years. Transplantation 2002;73:775-82.

47. Hariharan S, McBride MA, Cherikh WS, Tolleris CB, Bresnahan BA and Johnson CP. Posttransplant renal function in the first year predicts long-term kidney transplant survival. Kidney Int 2002;62:311-8.

48. Baboolal K, Jones GA, Janezic A, Griffiths DR and Jurewicz WA. Molecular and structural consequences of early renal allograft injury. Kidney Int 2002;61:686-96.

49. Hooff JP van, Boots JMM, Duijnhoven EM van and Christiaans MHL. Dosing and management guidelines for tacrolimus in renal transplant patients. Transplant Proc 1999;31 (S7A):54S-7.

50. Margreiter $\mathrm{R}$ for the European Tacrolimus vs Ciclosporin Microemulsion Renal Transplantation Study Group. Efficacy and safety of tacrolimus compared with ciclosporin microemulsion in renal transplantation: a randomised multicentre study. Lancet 2002;359: 741-6. 


\section{Chapter 8}

\section{Conversion from cyclosporin to tacrolimus improves quality-of-life indices, renal graft function and cardiovascular risk profile}

MA Artz, JMM Boots, G Ligtenberg, JI Roodnat, MHL Christiaans, PF Vos, P Moons, G Borm, LB Hilbrands 


\section{Abstract}

Long-term use of cyclosporin after renal transplantation results in nephrotoxicity and an increased cardiovascular risk profile. Tacrolimus may be more favourable in this respect. In this randomized controlled study in 124 renal transplant patients, the effects of conversion from cyclosporin to tacrolimus on renal function, cardiovascular risk factors, and perceived side-effects were investigated after a follow-up of two years.

After conversion from cyclosporin to tacrolimus, renal function remained stable, whereas continuation of cyclosporin was accompanied by a rise in serum creatinine from $142 \pm 48 \mu \mathrm{mol} / 1$ to $157 \pm 62 \mu \mathrm{mol} / 1 \quad(P<0.05$ comparing both groups). Conversion to tacrolimus resulted in a sustained reduction in systolic and diastolic blood pressure, and a sustained improvement in the serum lipid profile, leading to a reduction in the Framingham risk score from $5.7 \pm 4.3$ to $4.8 \pm 5.3$ $(P<0.05)$. Finally, conversion to tacrolimus resulted in decreased scores for occurrence of and distress due to side-effects.

In conclusion, conversion from cyclosporin to tacrolimus in stable renal transplant patients is beneficial with respect to renal function, cardiovascular risk profile, and side-effects. Therefore, for most renal transplant patients tacrolimus will be the drug of choice when long-term treatment with a calcineurin inhibitor is indicated. 


\section{Introduction}

Cyclosporin and tacrolimus are calcineurin inhibitors that are very effective in reducing the incidence of acute rejection after renal transplantation. However, cyclosporin-induced side effects, including nephrotoxicity, hypertension, and hyperlipidemia ${ }^{1}$ may contribute to the high cardiovascular morbidity in renal transplant patients ${ }^{2}$, and can also lead to an accelerated loss of graft function ${ }^{3,4}$. Tacrolimus has also been associated with nephrotoxicity, but several studies report an increased graft survival in patients using tacrolimus as initial immunosuppressive treatment ${ }^{5,6}$. Moreover, tacrolimus is associated with less hypertension and hyperlipidemia, thereby improving the cardiovascular risk profile ${ }^{1}$. However, a drawback of tacrolimus is the increased risk of developing diabetes mellitus ${ }^{5,6}$, which could antagonize the beneficial effect of reduced blood pressure and improved hyperlipidemia on the cardiovascular morbidity and mortality.

Many of the renal transplant patients that have been transplanted in the past two decades are using cyclosporin as maintenance immunosuppressive therapy. The question arises whether conversion from cyclosporin to tacrolimus in these chronic renal transplant patients will lead to an improved cardiovascular risk profile and whether renal graft function still can be improved.

In this prospective randomized controlled multi-center trial we compared the effects of conversion from cyclosporin to tacrolimus in stable chronic renal transplant patients on graft function and on the cardiovascular risk profile, expressed as the Framingham risk score. Also, we assessed the patients' perceived symptom experience associated with side effects of the immunosuppressive therapy, using the "modified transplant symptom occurrence and symptom distress scale" . The changes in several metabolic parameters during the first six months after conversion from cyclosporin to tacrolimus have been described in detail before ${ }^{8}$. In this paper, data on renal function, Framingham risk score, and side effects until the end of follow-up, at two years after randomisation, are presented.

\section{Material and methods}

\section{Patients}

To be eligible for the study the patients had to be at least one year posttransplantation with a stable endogenous creatinine clearance exceeding $20 \mathrm{ml} / \mathrm{min}$. Immunosuppressive treatment at enrollment had to include cyclosporin with trough levels ranging from 50 to $200 \mathrm{ng} / \mathrm{ml}$ and prednisone, either with or without azathioprine or mycophenolate mofetil. 


\section{Study protocol}

The study protocol has been described before ${ }^{8}$. In short, this prospective randomized controlled open-label multicenter trial was performed in four renal transplant centers. After stratification for center, patients were randomized to either continuation of cyclosporin or conversion to tacrolimus. Total follow-up was 24 months. Evaluation took place at baseline, at 3, 6, 12 and 24 months. Target drug trough levels were 50 to $200 \mathrm{ng} / \mathrm{ml}$ for cyclosporin and 5 to $8 \mathrm{ng} / \mathrm{ml}$ for tacrolimus. Adjustment of lipid-lowering drug treatment and antihypertensive treatment was only allowed under strict, predefined conditions. Blood pressure was measured in supine position manually after 5 minutes of rest and also using an automated device (Dinamap, Critikon Inc., Tampa, USA) during 30 minutes. The diagnosis of diabetes mellitus was based on fasting glucose levels, with a cut-off value of $7.0 \mathrm{mmol} / \mathrm{l}$, measured on two separate occasions, in accordance with the American Diabetes Association criteria9.

Framingham risk scores were calculated using baseline age, smoking behaviour, the presence of diabetes mellitus, fasting LDL-and HDL-cholesterol levels and the systolic and diastolic blood pressure ${ }^{10}$. The study was approved by the medical ethics committee of each participating center. All patients gave written informed consent.

\section{Laboratory procedures}

All blood was drawn after an overnight fast. Plasma cholesterol and (fasting) triglycerides were measured at a certified routine clinical chemistry laboratory on a Hitachi 747 analyser using reagents of Roche (no's: 337574 and 1361155, respectively). Cyclosporin levels were determined in whole blood with a fluorescence-polarization immune-assay (TDx/TDx FLx cyclosporin monoclonal whole blood assay, Abbott laboratories, Chicago, IL, USA) and tacrolimus levels were determined using a microparticle enzyme immune-assay (Imx Tacrolimus II Assay, Abbott Laboratories). The endogenous creatinine clearance was calculated using the creatinine excretion in a 24-hours urine sample.

\section{Assessment of symptom experience}

Symptom experience associated with side effects of the immunosuppressive therapy was measured using the "Modified Transplant Symptom Occurrence and Distress Scale" (MTSOSDS)7. This scale includes 38 symptoms associated with side effects of immunosuppressive medication (tacrolimus, cyclosporin, prednisone and azathioprine). The instrument measures symptom experience on a five-point rating scale from 0 (never occurring) to 4 (always occurring) for symptom occurrence, and from 0 (not at all distressing) to 4 (extremely distressing) for symptom distress, respectively. The following items were evaluated: acne, fragile skin, easy bruising, warts, skin rash, increased hair growth, 
hair loss, gingival hyperplasia, moon face, changed facial appearance, changed bodily appearance, swollen ankles, warmth in hands and feet, headache, poor vision, light insensitivity, tremor, paraesthesias, muscle weakness, muscle cramps, impotence / painful menstruation, decreased interest in sex, depression, agitation, anxiousness, mood swings, poor concentration, sleeplessness, hallucinations, nightmares, fatigue, increased appetite, poor appetite, abdominal pain, stomach complaints, diarrhoea, dysuria and back pain. The initial 29-item version of this instrument has been validated for the use in renal transplant patients7. The content validity of the updated instrument is based on a comprehensive literature review and has been tested by an expert panel (data on file).

\section{Statistical analysis}

Statistical Analysis was performed using the Statistical Product and Services Solutions (SPSS) package, version 11.0. Values are given as mean \pm standard deviation in case of a normal distribution or otherwise as median and interquartile range. For the serum lipids, blood pressure, glucose, $\mathrm{HbA}_{1} \mathrm{c}$, and the Framingham risk scores, the mean level during treatment was calculated for each patient by dividing the area under the curve by the treatment duration, since the cardiovascular risk is related to the cumulative exposure time to risk factors. For analysis of renal function the value at the end of follow-up was used. Analysis of the serum creatinine levels was performed after normalization by calculating the reciprocal value. Statistical analysis was performed using Analysis of Co-Variance (ANCOVA). Independent factors were randomisation-group, baseline value, and in case of serum lipids the use of a statin at baseline. Categorical values were analyzed using the chi-square test or Fisher's exact test when appropriate.

According to previous studies in the renal transplant population, symptom occurrence and symptom distress scores were analyzed by means of ridit analysis ${ }^{11}$. Ridits represent a probability measure Relative to an Identified Distribution, which can be analyzed parametrically. The ridit of a (sub)sample is always compared with the ridit of a chosen reference group and represents the probability that a randomly selected individual from that (sub)group scores higher on the response variable than a randomly selected individual of the reference group. The ridit of the reference group is by definition 0.500 . For calculation of symptom distress, those symptoms that never occurred were converted to missing values, in order to avoid anticipatory symptom distress 7 . In order to determine a rank order of most frequently occurring and most distressing symptoms at baseline, a ridit was calculated for each item, using the frequency distribution of all answers on all items at baseline as reference group. To calculate the evolution of the overall symptom occurrence and distress score in both the cyclosporin and the tacrolimus group, the reference group was constituted by the frequency distribution of the responses of all patients on all items at baseline. Student's t-test was used to compare the ridits obtained. 
All patients that were alive and not on renal replacement therapy were followed for renal function and blood pressure and these parameters were analyzed on intention-to-treat base. In the patients who died or who started on renal replacement therapy, the last serum creatinine value obtained before death or renal replacement therapy was used for the analysis. The metabolic parameters and the symptom experiences were analyzed in the patients who remained on study medication during follow-up. A P-value of less than 0.05 (two-sided) was considered statistically significant.

\section{Results}

A total of 124 patients were included in the study, 64 were converted to tacrolimus and 60 remained on cyclosporin. Mean time after transplantation was 6.6 \pm 3.7 years for the tacrolimus group and $5.7 \pm 2.7$ years for the cyclosporin group. The demographic data have been described before ${ }^{8}$. At baseline, the cyclosporin level was $129 \pm 42 \mathrm{ng} / \mathrm{ml}$ in the cyclosporin group and $130 \pm 42 \mathrm{ng} / \mathrm{ml}$ in the tacrolimus group. During follow-up, the cyclosporin level remained unchanged in the cyclosporin group. In the tacrolimus group the tacrolimus level was $7.2 \pm 2.0 \mathrm{ng} / \mathrm{ml}$ at three months and $6.6 \pm 1.8 \mathrm{ng} / \mathrm{ml}$ at two years after randomisation. Baseline prednisone dose was equal in both groups $(0.10 \pm 0.04$ $\mathrm{mg} / \mathrm{kg})$. Baseline azathioprine dose was $0.88 \pm 0.40 \mathrm{mg} / \mathrm{kg} \quad(\mathrm{n}=10)$ in the tacrolimus group and $0.95 \pm 0.47 \mathrm{mg} / \mathrm{kg}(\mathrm{n}=15)$ in the cyclosporin group. In the cyclosporin group, two patients used mycophenolate mofetil $2000 \mathrm{mg} / \mathrm{day}$. Prednisone was stopped in two patients, and they were both withdrawn from the study. Azathioprine was stopped in one patient in the tacrolimus group and in one patient in the cyclosporin group.

The study protocol was not completed until the end of follow-up in 17 patients in the tacrolimus group and in 15 patients of the cyclosporin group (Table 8.1). In the tacrolimus group, two patients reached end-stage renal disease (ESRD), due to biopsy-proven chronic allograft nephropathy. In the cyclosporin group one patient, known to have recurrent membranous glomerulonephritis, reached ESRD. In each group, one patient was withdrawn from the study due to deteriorating renal function associated with chronic allograft nephropathy (tacrolimus group) and cyclosporin nephrotoxicity (cyclosporin group), respectively. 
Table 8.1 Patients not completing the study.

\begin{tabular}{lcc}
\hline & Cyclosporin & Tacrolimus \\
\hline Death of patient & $6(10 \%)$ & $2(3.1 \%)$ \\
cardiovascular & 3 & 2 \\
malignancy & 1 & 0 \\
infection & 1 & 0 \\
pulmonary embolism & 1 & 0 \\
End-stage renal disease & $1(1.7 \%)$ & $2(3.1 \%)$ \\
Withdrawal of study medication & & \\
deterioration of renal function & $1(1.7 \%)$ & $1(1.6 \%)$ \\
intractable hypertension & $2(3.3 \%)$ & $0(0 \%)$ \\
new-onset DM & $0(0 \%)$ & $2(3.1 \%)$ \\
dysregulation of pre-existent DM & $0(0 \%)$ & $2(3.1 \%)$ \\
other side effects & $5(8.3 \%)$ & $8(12.5 \%)$ \\
& & $17(26.6 \%)$ \\
Total & $15(25.0 \%)$ & 17 \\
\hline
\end{tabular}

\section{Renal function}

Prior to inclusion, all patients had a stable serum creatinine concentration. During the two-year follow-up period, serum creatinine increased in the cyclosporin group from $142 \pm 48$ to $157 \pm 62 \mu \mathrm{mol} / 1(P<0.001)$, while it remained stable in the tacrolimus group $(139 \pm 32 \mu \mathrm{mol} / 1$ at baseline and $145 \pm 56 \mu \mathrm{mol} / 1$ at 24 months, $P=0.26, P<0.05$ for comparison between groups, Figure 8.1). In the cyclosporin group the endogenous creatinine clearance decreased from $59 \pm 26 \mathrm{ml} / \mathrm{min}$ to $49 \pm 22 \mathrm{ml} / \mathrm{min}(\mathrm{n}=50 ; P<0.001)$, whereas renal function remained stable in the tacrolimus group $(60 \pm 22 \mathrm{ml} / \mathrm{min}$ and $64 \pm 33 \mathrm{ml} / \mathrm{min}, \mathrm{n}=58, P<0.01$ for comparison between groups).

In the cyclosporin group, 30 patients $(50 \%)$ had a more than $10 \%$ rise in serum creatinine level during the two years of follow-up, as opposed to 15 patients $(23.4 \%)$ in the tacrolimus group, while six patients $(10 \%)$ in the cyclosporin group had a more than $10 \%$ improvement in serum creatinine level during follow-up, compared to 20 patients $(31 \%)$ in the tacrolimus group $(P<0.001)$.

Proteinuria did not significantly change in either group during follow-up (median baseline value $0.2(0-4.7) \mathrm{g} / 24$ hours in the cyclosporin group and $0.2(0-3.6) \mathrm{g} / 24$ hours in the tacrolimus group). 


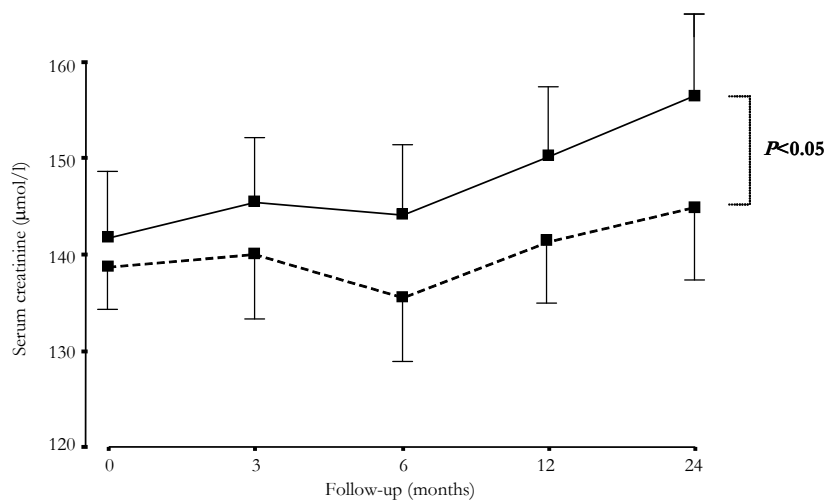

Figure 8.1 Serum creatinine (mean and SEM) on cyclosporin (- ${ }_{-}$) and on tacrolimus (-------).

\section{Cardiovascular risk factors}

In the tacrolimus group, the systolic and diastolic blood pressure (manually measured) were reduced from $144 \pm 20 \mathrm{mmHg}$ to $137 \pm 21 \mathrm{mmHg}(P<0.001)$, and from $84 \pm 11 \mathrm{mmHg}$ to $80 \pm 9 \mathrm{mmHg}(P<0.01)$, respectively (Figure 8.2$)$. As a result, blood pressure in the tacrolimus group was significantly reduced when compared to the cyclosporin group $(P<0.01)$. The dynamap blood pressure readings essentially showed the same results (data not shown).
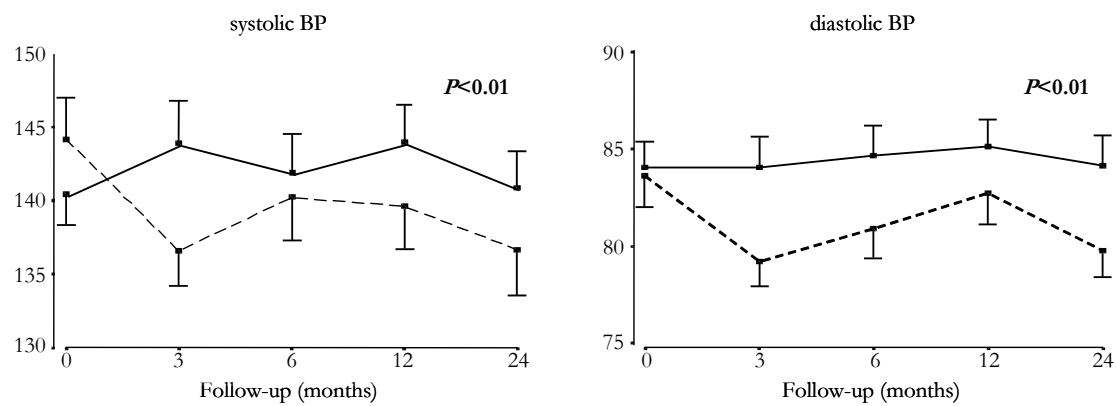

Figure 8.2 Systolic and diastolic blood pressure ( $\mathrm{mmHg}$, mean and SEM) on cyclosporin $(-)$ and on tacrolimus (-------). P<0.01 comparing the AUC on cyclosporin and on tacrolimus for both systolic and diastolic blood pressure.

In the cyclosporin group, the systolic blood pressure was reduced by at least $12 \mathrm{mmHg}$ in 15 patients (25\%), as opposed to 28 patients (44\%) in the tacrolimus group $(P<0.05)$. The diastolic blood pressure was reduced by at least $12 \mathrm{mmHg}$ in 
$5(8 \%)$ cyclosporin-treated patients and $17(27 \%)$ tacrolimus-treated patients $(P<0.01)$.

The number of antihypertensive drugs per patient in the cyclosporin group was $1.9 \pm 1.0$ at baseline and $2.3 \pm 1.1$ at two-year follow-up. In the tacrolimus group the number of antihypertensive drugs was $2.0 \pm 1.1$ at baseline and $2.3 \pm 1.1$ at two-year follow-up (no differences between groups).

Serum total and LDL-cholesterol, and serum triglycerides were significantly reduced in the tacrolimus group, from $5.9 \pm 0.8 \mathrm{mmol} / 1$ to $5.2 \pm 0.8 \mathrm{mmol} / 1$ $(P<0.001)$, from $3.6 \pm 0.8 \mathrm{mmol} / 1$ to $3.1 \pm 0.8 \mathrm{mmol} / 1 \quad(P<0.001)$, and from $2.1 \pm 1.0 \mathrm{mmol} / 1$ to $1.7 \pm 1.1 \mathrm{mmol} / 1 \quad(P<0.05)$, respectively $(P<0.001$ for comparison between groups for total and LDL-cholesterol and $P<0.01$ for triglycerides, Figure 8.3). Serum HDL-cholesterol level remained unchanged in both groups. The use of a statin did not influence the changes in the serum lipid levels. Commencement of statin treatment or an increase in the dosage according to predefined guidelines, was necessary in three patients of the tacrolimus group and in ten patients of the cyclosporin group. After conversion to tacrolimus, the statin was stopped in one patient, whereas this did not occur in the cyclosporin group.

The fasting glucose level and the $\mathrm{HbA}_{1} \mathrm{c}$ level were analyzed for diabetic and nondiabetic patients separately, since in diabetic patients both glucose and $\mathrm{HbA}_{1} \mathrm{c}$ levels are influenced by dietary (non)-compliance and changes in medication. Fasting glucose and $\mathrm{HbA}_{1} \mathrm{c}$ levels did not change in either group during follow-up (Table 8.2). In the cyclosporin group, three patients developed new-onset diabetes mellitus versus four patients in the tacrolimus group. In the diabetic patients of the cyclosporin group $(n=8)$, the anti-diabetic treatment remained unchanged in five patients. In two patients, the dosage of anti-diabetic medication had to be increased and one patient was started on insulin. In the diabetic patients of the tacrolimus group $(\mathrm{n}=8)$, the anti-diabetic medication remained unchanged in three patients. In two patients, the dosage of anti-diabetic medication had to be increased and two patients were started on insulin. In one patient, the dosage of insulin was reduced.

The Framingham risk score was significantly reduced from $5.7 \pm 4.3$ at baseline to $4.8 \pm 5.3$ after two years in the tacrolimus group $(P<0.05)$, and remained unchanged in the cyclosporin group (6.0 \pm 3.1 at baseline and $6.2 \pm 3.6$ at 24 months, $P<0.05$ for comparison between groups, Figure 8.4). The use of a statin at baseline did not influence the change in Framingham risk score upon conversion to tacrolimus. 
Table 8.2 Glucose regulation on cyclosporin versus tacrolimus.

\begin{tabular}{|c|c|c|c|c|c|c|}
\hline & & \multicolumn{2}{|c|}{ Cyclosporin } & \multicolumn{2}{|c|}{ Tacrolimus } & \\
\hline & & baseline & 24 months & baseline & 24 months & \\
\hline \multicolumn{2}{|c|}{ Glucose $(\mathrm{mmol} / \mathrm{l})$ non-diabetics } & $5.0(4.1-6.2)$ & $5.2(4.0-7.0)$ & $5.0(3.4-7.6)$ & $5.2(4.0-7.1)$ & N.S. \\
\hline & $\begin{array}{l}\text { diabetics } \\
(\mathrm{n}=16)\end{array}$ & $9.5(6.5-11.8)$ & $9.7(5.5-4.0)$ & $7.7(5.8-11.6)$ & $7.9(6.0-4.1)$ & N.S. \\
\hline \multirow[t]{2}{*}{$\mathrm{HbA}_{1} \mathrm{c}(\%)^{\mathrm{a}}$} & $\begin{array}{l}\text { non-diabetics } \\
(\mathrm{n}=31)\end{array}$ & $5.5 \pm 0.4$ & $5.7 \pm 0.3$ & $5.5 \pm 0.4$ & $5.6 \pm 0.4$ & N.S. \\
\hline & $\begin{array}{l}\text { diabetics } \\
(\mathrm{n}=16)\end{array}$ & $7.9 \pm 0.6$ & $8.0 \pm 0.8$ & $8.8 \pm 1.7$ & $7.5 \pm 1.7$ & N.S. \\
\hline
\end{tabular}

N.S.: no significant differences between groups. ${ }^{a}=\mathrm{HbA}_{1} \mathrm{c}$ was determined in all patients from one participating center $(n=35)$, and in the remaining three centers in the diabetic patients only.
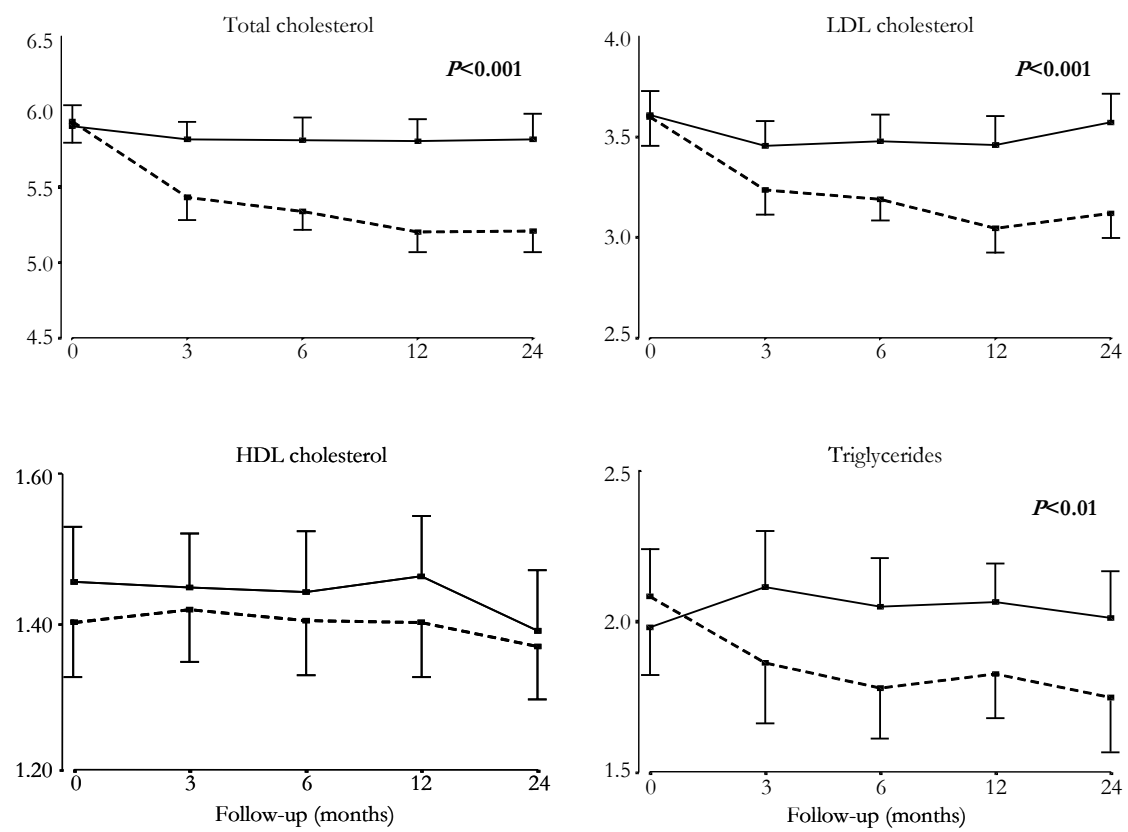

Figure 8.3 Serum lipid levels and serum triglyceride levels ( $\mathrm{mmol} / \mathrm{l}$, mean and SEM) on cyclosporin (- $\longrightarrow$ and on tacrolimus (-------). $P<0.001$ comparing the AUC on cyclosporin and tacrolimus for total and LDL-cholesterol, and $P<0.01$ for the serum triglyceride levels. 


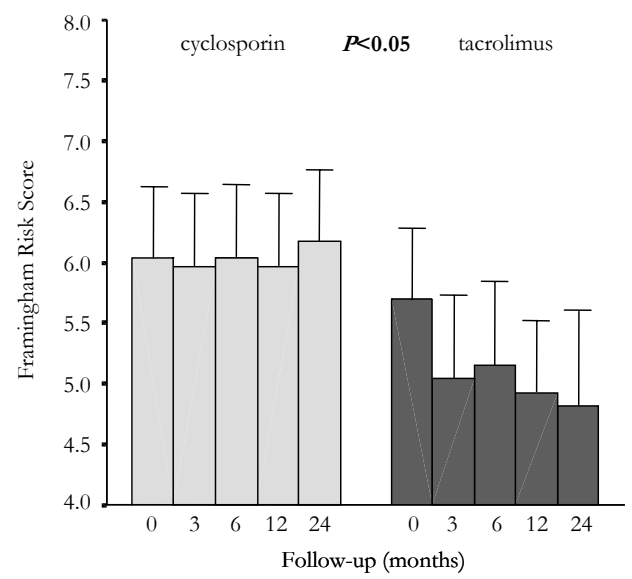

Figure 8.4 Framingham risk score (mean and SEM) on cyclosporin and on tacrolimus. $P<0.05$ comparing the AUC on cyclosporin and on tacrolimus.
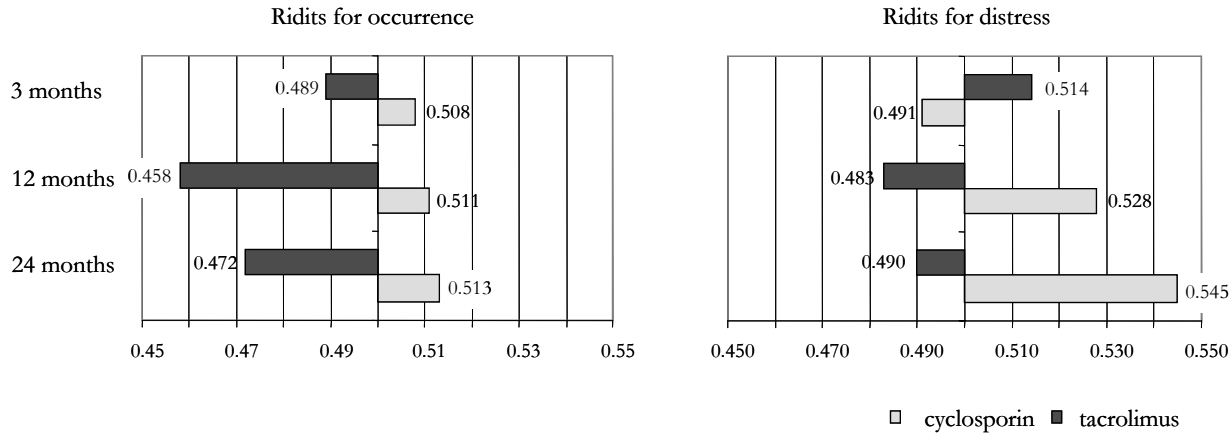

Figure 8.5 Evolution of overall symptom occurrence and symptom distress at 3, 12 and 24 months. $P<0.001$ for all comparisons with baseline values.

\section{Symptom experience associated with side effects of immunosuppressive therapy}

In the tacrolimus group, eight patients were taken off tacrolimus due to the following side effects: hair loss $(n=2)$, headache $(n=2)$, diarrhea $(n=1)$, palpitations $(n=1)$, tremor $(n=1)$ and toxic exanthema $(n=1)$. In the cyclosporin group, two patients were withdrawn from the study due to gingival hyperplasia $(n=1)$ and to severe osteoporosis for which prednisone was stopped $(n=1)$. In addition, two patients wanted to be taken off cyclosporin and one patient was converted from cyclosporin to azathioprine because of her wish to become pregnant. 
The ten most frequently occurring side effects at baseline were in decreasing order of frequency: increased hair growth, fatigue, bruises, excessive appetite, poor concentration, changed bodily appearance, poor vision, fragile skin, swollen ankles, and insomnia. The ten most distressing side effects at baseline were: impotence for men and painful menstruation for women, fragile skin, insomnia, warts, poor concentration, back pain, muscle weakness, stomach complaints, fatigue and nightmares. The item "hallucinations" was excluded from the analysis of symptom distress since it occurred in only one patient.

The overall ridit for symptom occurrence evolved significantly after randomisation, both in the cyclosporin and in the tacrolimus group. A significant increase $(P<0.0001)$ in symptom occurrence was observed in patients on cyclosporin at 3,12 and 24 months after baseline measurement (Figure 8.5). Conversely, patients who were converted to tacrolimus experienced a significant decrease $(P<0.0001)$ of symptom occurrence compared to baseline level. The ridit of 0.458 for the tacrolimus group at 12 months means that a randomly selected patient from that group has a chance of $45.8 \%$ to score higher than a randomly selected patient at baseline (reference group). This chance of $45.8 \%$ is significantly lower than the chance of $50 \%$ (ridit 0.500 ) inherent to the reference group.

For symptom distress, the overall ridit of the tacrolimus group at three months after conversion was significantly increased $(P<0.0001)$, while the ridit of the cyclosporin group was decreased. Nonetheless, at 12 and 24 months, patients of the tacrolimus group reported significantly less, and cyclosporin patients significantly more symptom distress compared to the baseline level.

\section{Discussion}

This randomized controlled study demonstrates that after conversion from cyclosporin to tacrolimus in stable renal transplant patients graft function remains stable as opposed to a gradual deterioration of graft function when continuing on cyclosporin. Furthermore, replacement of cyclosporin by tacrolimus is followed by an improvement in cardiovascular risk profile, which is sustained during a twoyear follow-up period. In addition, the occurrence of and distress due to sideeffects is reduced.

In patients using tacrolimus as part of the initial immunosuppressive treatment, the incidence of acute rejections and of steroid-resistant rejections is reduced, compared to patients using cyclosporin ${ }^{5,6}$, without affecting one-year graft and patient survival. Acute rejection is a major risk factor for developing chronic allograft nephropathy ${ }^{12}$, and several studies have suggested that tacrolimus may indeed improve long-term graft survival rates. Data from the UCLA-UNOS (United Network for Organ Sharing) kidney transplant registry showed a graft half-life of approximately 14 years on tacrolimus and eight to nine years on 
cyclosporin and other immunosuppressive agents ${ }^{13}$. Since this first report, several other studies have provided evidence that compared to an initial immunosuppressive protocol including cyclosporin, a protocol including tacrolimus may increase graft half-life ${ }^{6,14}$. The influence of tacrolimus on graft survival however is not unequivocal. In the analysis of Hariharan et al., the use of tacrolimus did not contribute to the recent improvements in graft survival ${ }^{15}$.

The putative beneficial effect of tacrolimus on graft function and ultimately also graft survival, may be related to several favourable effects of tacrolimus compared to cyclosporin. Chronic allograft nephropathy (CAN) is the leading cause of late graft failure and may result from chronic rejection as well as from nonimmunologic processes like calcineurin inhibitor nephrotoxicity and graft vascular disease ${ }^{16}$. The occurrence of an acute rejection is the strongest predictor for the development of chronic rejection ${ }^{16}$, and tacrolimus reduces the incidence of acute rejections. Secondly, tacrolimus may be less nephrotoxic than cyclosporin, thus contributing to the prolonged graft survival. The induction of profibrotic cytokines like osteopontin ${ }^{17}$ and transforming growth factor (TGF)- $\beta^{18}$ by cyclosporin is one of the pathophysiologic mechanisms in chronic cyclosporin nephropathy. Although TGF- $\beta$ is also involved in chronic tacrolimus nephropathy ${ }^{19}$, several studies suggested a reduced renal mRNA-expression of TGF- $\beta$ and other profibrotic genes on tacrolimus versus cyclosporine ${ }^{20,21}$. Finally, hyperlipidemia and hypertension are important non-immunologic factors contributing to chronic allograft nephropathy ${ }^{22-24}$. In this study, we observed reductions in serum LDL-cholesterol, triglyceride levels and blood pressure, that were apparent at three months after conversion to tacrolimus and persisted until the end of follow-up. This sustained improvement in serum lipid levels as well as in blood pressure is likely to be of benefit in maintaining graft function.

Mayer et al. reported a reduced incidence of CAN after the use of tacrolimus as part of the initial immunosuppression ${ }^{6}$. It is of interest to see that even in our study population, consisting of patients that were $6.1 \pm 3.2$ years posttransplantation, the course of the graft function can still be improved by conversion to tacrolimus. In this respect, the question can be raised whether equipotent dosages of tacrolimus and cyclosporin were used. In our population, consisting mainly of Caucasian patients, the dosage of tacrolimus was sufficient to prevent acute rejections in all patients. The mean tacrolimus trough level (about $7 \mathrm{ng} / \mathrm{ml}$ ) as well as the mean cyclosporin trough level (about $130 \mathrm{ng} / \mathrm{ml}$ ) were within the ranges that have been advised for maintenance immunosuppressive therapy after renal transplantation ${ }^{25}$. The tacrolimus levels in our patients were similar to the levels found in the study of Mayer et al. ${ }^{6}$. A second question is whether the way of cyclosporin monitoring could have influenced the results. When this study was designed, it was common practice to use the trough level $\left(\mathrm{C}_{0}\right)$ for monitoring. However, it is now recognized that the area-under-the-curve for the first four hours post-dose $\left(\mathrm{AUC}_{0-4}\right)$ is superior to the $\mathrm{C}_{0}$ level of cyclosporin in 
predicting the risk for acute rejection and cyclosporin-induced nephrotoxicity ${ }^{26}$, and the two-hours post-dose $\left(\mathrm{C}_{2}\right)$ level of cyclosporin is an accurate surrogate marker of the $\mathrm{AUC}_{0-4}$ cyclosporin level2 ${ }^{27}$. Prospective studies on the long-term effects of replacing $\mathrm{C}_{0}$ monitoring by $\mathrm{C}_{2}$ monitoring in maintenance renal transplant patients are limited. Available date suggest that dose reduction in overexposed patients leads to improvements in renal function and blood pressure ${ }^{28}$. However, it is currently unclear whether $C_{2}$ monitoring can result in comparable effects on graft function, blood pressure and serum lipids as we have observed after conversion from cyclosporin to tacrolimus.

We previously already showed that conversion from cyclosporin to tacrolimus resulted in a reduction in systolic and diastolic blood pressure, and in an improvement in several metabolic cardiovascular risk factors, including total and LDL-cholesterol levels, triglyceride levels, fibrinogen levels, and the oxidizability of the LDL-particles ${ }^{8}$. We now demonstrate that this improvement in the cardiovascular risk profile is sustained. Although the reductions in systolic and diastolic blood pressure are modest, in patients with a high risk of developing cardiovascular disease, like renal transplant patients, the numbers-needed-to-treat to prevent cardiovascular disease or cardiovascular death are relatively small. For example, in case of a reduction of the systolic blood pressure with $12 \mathrm{~mm} \mathrm{Hg}$, the numbers-needed-to-treat to prevent cardiovascular disease is only ten ${ }^{29}$.

During the two years of follow-up, conversion from cyclosporin to tacrolimus did not affect plasma fasting glucose and $\mathrm{HbA}_{1} \mathrm{c}$ levels, and there was no significant difference in the occurrence of new-onset diabetes mellitus. The diabetogenic effect of tacrolimus is dose-related, and the risk of developing diabetes mellitus is highest in the initial period after transplantation when high trough levels are reached $^{30}$, whereas at standard maintenance trough levels, no difference between cyclosporin and tacrolimus could be determined with regard to their diabetogenic properties $^{31}$.

The risk of developing diabetes mellitus after conversion to tacrolimus appears to be restricted to patients who already experience a disturbed glucose tolerance prior to therapy $y^{5,32}$.

The improvements in lipid profile and blood pressure after conversion to tacrolimus resulted in a sustained reduction of the Framingham risk score. This cardiovascular risk score was developed in a predominantly Caucasian population without specific underlying diseases ${ }^{10}$ and has been validated in renal transplant patients, where it was shown to predict ischemic cardiovascular events, though underestimating the absolute risk, especially related to diabetes mellitus ${ }^{33}$. In the general population, the observed reduction in Framingham risk score upon conversion to tacrolimus would imply a relative reduction of approximately $20 \%$ in the estimated risk for coronary heart disease over a period of ten years. Although it is likely that renal transplant patients will also benefit from the 
reduced cardiovascular risk score, it is difficult to extrapolate the risk rates to this specific population.

After conversion from cyclosporin to tacrolimus, the occurrence of symptoms associated with side-effects of the immunosuppressive regimen decreased significantly. This was also observed for symptom distress, except for the measurement at three months after conversion. These findings demonstrate the beneficial effect of conversion to tacrolimus in terms of patients' symptom experience. Patients' subjective appraisal of symptoms associated with side-effects is very important for their quality-of-life ${ }^{34}$, and is also of great importance for the motivation to comply with the medication regimen ${ }^{35}$. Several studies have demonstrated a relation between distressing symptoms and subsequent noncompliance with immunosuppressive medication ${ }^{36,37}$. Further analysis of symptom occurrence and symptom distress at item level will provide a more detailed picture of the evolution of individual symptoms after conversion from cyclosporin to tacrolimus.

In summary, conversion from cyclosporin to tacrolimus in stable chronic renal transplant patients has a beneficial effect on renal graft function, results in a sustained improvement in the cardiovascular risk profile and reduces the frequency and distress of side-effects. In our opinion, for most renal transplant patients tacrolimus will be the drug of choice when long-term treatment with a calcineurin inhibitor is indicated. 


\section{References}

1. Ligtenberg G, Hené RJ, Blankestijn PJ and Koomans HA. Cardiovascular risk factors in renal transplant patients: Cyclosporin A versus tacrolimus. J Am Soc Nephrol 2001;12:368-73.

2. Woo YM, Jardine AG, Clark AF, MacGregor MS, Bowman AW, Macpherson SG, Briggs JD, Junor BJ, McMillan MA and Rodger RS. Early graft function and patient survival following cadaveric renal transplantation. Kidney Int 1999;55:692-9.

3. Dimény E, Wahlberg J, Lithell $\mathrm{H}$ and Fellström B. Hyperlipidaemia in renal transplantation risk factor for long-term graft outcome. Eur J Clin Invest 1995;25:574-83.

4. Peschke B, Scheuermann, Geiger H, Bölscher S, Kachel H-G and Lenz T. Hypertension is associated with hyperlipidemia, coronary heart disease and chronic graft failure in kidney transplant recipients. Clin Nephrol 1999;51:290-5.

5. Pirsch JD, Miller J, Deierhoi MH, Vincenti F and Filo RS for the FK506 Kidney Transplant Study Group. A comparison of tacrolimus (FK506) and cyclosporine for immunosuppression after cadaveric renal transplantation. Transplantation 1997;63:977-83.

6. Mayer AD for the European Tacrolimus Multicentre Renal Study Group. Chronic rejection and graft half-life: Five year follow-up of the European Tacrolimus Multicenter Renal Study. Transplant Proc 2002;34:1491-2.

7. Moons P, Geest S de, Versteven K, Abraham I, Vlaminck H, Moens G and Waer M. Psychometric properties of the "Modified Transplant Symptom Occurrence and Symptom Distress Scale". J Nurs Meas 2001;9:115-34.

8. Artz MA, Boots JMM, Ligtenberg G, Roodnat JI, Christiaans MHL, Vos PF, Blom HJ, Sweep FCGJ, Demacker PNM and Hilbrands LB. Improved cardiovascular risk profile and renal function in renal transplant patients after randomized conversion from cyclosporine to tacrolimus. J Am Soc Nephrol 2003;14:1880-8.

9. Expert Committee on the Diagnosis and Classification of Diabetes Mellitus. American Diabetes Association: clinical practice recommendations 2002. Diabetes Care 2002;25 (S1): S1-147.

10. Wilson PWF, D'Agostino RB, Levy D, Belanger AM, Silbershatz H and Kannel WB. Prediction of coronary heart disease using risk factor categories. Circulation 1998;97:1837-47.

11. Sermeus W and Delesie L. Ridit analysis on ordinal data. West J Nurs Res 1996;18:351-9.

12. Hariharan S, Johnson CP, Bresnahan BA, Taranto SE, McIntosh MJ and Stablein D. Improved graft survival after renal transplantation in the United States, 1988 to 1996. N Engl J Med 2000;342:605-12.

13. Gjertson DW, Cecka JM and Terasaki PI. The relative effects of FK506 and cyclosporine on short- and long-term kidney graft survival. Transplantation 1995;60:1384-8.

14. Vincenti F, Jensik SC, Filo RS, Miller J and Pirsch J. A long-term comparison of tacrolimus (FK506) and cyclosporine in kidney transplantation: evidence for improved allograft survival at five years. Transplantation 2002;73:775-82.

15. Hariharan S, McBride MA, Cherikh WS, Tolleris CB, Bresnahan BA and Johnson CP. Posttransplant renal function in the first year predicts long-term kidney transplant survival. Kidney Int 2002;62:311-8.

16. Hariharan S. Long-term kidney transplant survival. Am J Kidney Dis 2001;38(S6): S44-50. 
17. Pichler RH, Franceschini N, Young BA, Hugo C, Andoh TF, Burdman EA, Shankland SJ, Alpers CE, Bennett WM and Couser WG. Pathogenesis of cyclosporine nephropathy: roles of angiotensin II and osteopontin. J Am Soc Nephrol 1995;6:1186-96.

18. Shihab FS, Andoh TF, Tanner AM, Noble NA, Border WA, Franceschini N and Bennett WM. Role of transforming growth factor-beta 1 in experimental chronic cyclosporine nephropathy. Kidney Int 1996;49:1141-51.

19. Shihab FS, Bennett WM, Tanner AM and Andoh TF. Mechanism of fibrosis in experimental tacrolimus nephrotoxicity. Transplantation 1997;64:1829-37.

20. Mohamed MAS, Robertson H, Booth TA, Balupuri S, Kirby JA and Talbot D. TGF- $B$ expression in renal transplant biopsies. A comparative study between cyclosporin-A and tacrolimus. Transplantation 2000;69:1002-5.

21. Bicknell GR, Shaw WJA, Pringle JH, Furness PN and Nicholson ML. Differential effects of cyclosporin and tacrolimus on the expression of fibrosis-associated genes in isolated glomeruli from renal transplants. Br J Surg 2000;87:1569-75.

22. Opelz G, Wujciak T, and Ritz E for the Collaborative Transplant Study. Association of chronic kidney graft failure with recipient blood pressure. Kidney Int 1998;53:217-22.

23. Ponticelli C. Progression of renal damage in chronic rejection. Kidney Int 2000;57(S75):S62-70.

24. Carvalho MF and Soares V. Hyperlipidemia as a risk factor of renal allograft function impairment. Clin Transplant 2001;15:48-52.

25. Oellerich M, Armstrong VW, Schutz E and Shaw LM. Therapeutic drug monitoring of cyclosporine and tacrolimus. Update on Lake Louise Consensus Conference on cyclosporin and tacrolimus. Clin Biochem 1998;31:309-16.

26. Mahalati K, Belitsky P, Sketris I, West K and Panek R. Neoral monitoring by simplified sparse sampling area under the concentration-time curve: its relationship to acute rejection and cyclosporine nephrotoxicity early after kidney transplantation. Transplantation 1999;68:55-62.

27. Levy GA. C2 monitoring strategy for optimising cyclosporin immunosuppression from the Neoral formulation. BioDrugs 2001;15:279-90.

28. Cole E, Maham N, Cardella C, Cattran D, Fenton S, Hamel J, O'Grady C and Smith R. Clinical benefits of neoral $\mathrm{C} 2$ monitoring in the long-term management of renal transplant recipients. Transplantation 2003;75:2086-90.

29. Ogden LG, He J, Lydick E and Whelton PK. Long-term benefit of lowering blood pressure in hypertensive patients according to the JNC VI risk stratification. Hypertension 2000;35:539-43.

30. Weir MR and Fink JC. Risk for posttransplant diabetes mellitus with current immunosuppressive medications. Am J Kidney Dis 1999;34:1-13.

31. Duijnhoven EM van, Christiaans MHL, Boots JMM, Nieman FHR, Wolffenbuttel BHR and Hooff JP van. Glucose metabolism in the first 3 years after renal transplantation in patients on tacrolimus versus cyclosporine-based immunosuppression. J Am Soc Nephrol 2002;13:213-20.

32. Duijnhoven EM van, Boots JMM, Christiaans MHL, Wolffenbuttel BHR and Hooff JP van. Influence of tacrolimus on glucose metabolism before and after renal transplantation: a prospective study. J Am Soc Nephrol 2001;12:583-8.

33. Kasiske BL, Chakkera HA and Roel J. Explained and unexplained ischemic heart disease risk after renal transplantation. J Am Soc Nephrol 2000;11:1735-43.

34. Testa MA and Simonson DC. Assessment of quality-of-life outcomes. N Engl J Med 1996; 334:835-40. 
35. Leventhal H, Diefenbach $M$ and Leventhal EA. Illness cognition: using common sense to understand treatment adherence and affect cognition interactions. Cogn Ther Res 1992;16: 143-63.

36. Geest S de, Borgermans L, Gemoets H, Abraham I, Vlaminck H, Evers G and Vanrenterghem Y. Incidence, determinants, and consequences of subclinical noncompliance with immunosuppressive therapy in renal transplant recipients. Transplantation 1995;59:340-7.

37. Didlake RH, Dreyfus K, Kerman RH, Buren CT van and Kahan BD. Patient noncompliance: a major cause of late graft failure in cyclosporine-treated renal transplants. Transplant Proc 1988; 20(S3):63-9. 


\section{Chapter 9}

Effect of immunosuppressive agents on long-term survival of renal transplant recipients: focus on the cardiovascular risk

JMM Boots, MHL Christiaans, JP van Hooff

Drugs 2004;64(18):2047-2073 


\section{Summary}

In the control of acute rejection, attention is being focused more and more on the long-term side effects of the immunosuppressive agents used. Since cardiovascular disease is the main cause of death in renal transplant recipients, optimal control of cardiovascular risk factors is essential in the long-term management of these patients. Unfortunately, several commonly used immunosuppressive drugs interfere with the cardiovascular system. In this review, the cardiovascular adverse effects of the immuosuppressive agents currently used for maintenance immunosuppression are thoroughly discussed.

Optimizing immunosuppression means finding a balance between efficacy and safety. Corticosteroids induce endothelial dysfunction, hypertension, hyperlipidemia, and diabetes mellitus, and impair fibrinolysis. The use of corticosteroids in transplant recipients is undesirable, not only because of their cardiovascular effects, but also because they induce such adverse effects as osteoporosis, obesity, and atrophy of the skin and vessel wall. Calcineurin inhibitors are the most powerful agents for maintenance immunosuppression. The calcineurin inhibitor cyclosporin A not only induces these same adverse effects as corticosteroids but is also nephrotoxic. Tacrolimus has a more favourable cardiovascular risk profile than cyclosporin and is also less nephrotoxic. It has little or no effect on blood pressure and serum lipids; however its diabetogenic effect is more prominent in the period immediately following transplantation, although at maintenance dosages, the diabetogenic effect appears to be comparable to that of cyclosporin. The diabetogenic effect of tacrolimus can be managed by reducing the dose of tacrolimus and early corticosteroid withdrawal. The effect of tacrolimus on endothelial function has not been completely elucidated. The proliferation inhibitors azathioprine and mycophenolate mofetil (MMF) have little effect on the cardiovascular system. Yet, indirectly, by inducing anemia, they may lead to left ventricular hypertrophy. MMF is an attractive alternative to azathioprine because of its higher potency and possible lower risk of malignancies. Sirolimus also induces anemia, but may be promising because of its antiproliferative features. Whether the hyperlipidemia induced by sirolimus counteracts its beneficial effects is, as yet, unknown. It may be combined with MMF, however, initial attempts resulted in severe mouth ulcers. 


\section{Introduction}

When evaluating the success of organ transplantation, many studies focus on the initial results, especially the risk of acute rejection and the short-term patient and graft survival. For most transplanted organs like the heart, the lung, or the liver, this approach seems reasonable, since the survival of the patient is almost completely dependent on the survival of the graft. For transplantation of the small bowel or the pancreas, this is not the case, but experience with small bowel transplantation is limited and pancreas transplant recipients belong to a selected risk group with an increased risk of mortality from diabetes mellitus. This review focuses on renal transplant recipients.

It has long been debated whether renal transplantation, in addition to improving patient quality of life, also offers a survival benefit over continuation of dialysis. Since transplantation is usually performed in the best candidates, a proper control group is difficult to compose. Nevertheless, recent studies have made it clear that transplantation improves survival ${ }^{1,2}$, even in recipients of kidneys derived from marginal donors ${ }^{3}$. The main causes of mortality after renal transplantation are infections, malignancies, and cardiovascular disease ${ }^{4,5}$. The development of infections and malignancies is, in general, more dependent upon the immunosuppressive load than upon the individual agents administered. Since cardiovascular disease is the main cause of mortality in renal transplantation ${ }^{4,5}$, this review focuses on the cardiovascular risk posed by the different immunosuppressive agents commonly used in renal transplantation.

The immunosuppressive agents currently used to prevent rejection after transplantation include corticosteroids, inhibitors of lymphocyte proliferation (azathioprine (AZA) and mycophenolate mofetil (MMF)), calcineurin inhibitors (cyclosporin A (CsA) and tacrolimus (TAC)), target of rapamycin (TOR) inhibitors (rapamycin or sirolimus (RAPA)), mono- or polyclonal antibodies against $\mathrm{T}$ lymphocytes (anti-thymocyte globulin (ATG)) or the T-cell receptor CD 3 (OKT-3), and antibodies against the $\alpha$-chain of the interleukin (IL)-2 receptor (CD 25) (daclizumab and basiliximab). At present, a number of other agents are being developed, such as leflunomide, which is used in rheumatoid arthritis, FK 778 (meflunomide: an active metabolite of leflunomide), everolimus (a derivative of sirolimus), and FTY720. At the moment, experience with these compounds is limited in renal transplantation; therefore, they are not discussed further in this review. Mizoribine is used only in Japan ${ }^{6}$, and is, therefore, not considered either.

Antibodies are used either as induction therapy or for treatment of acute rejections, especially in the case of corticosteroid resistance. There are no data available on these compounds with regard to their effect on cardiovascular risk 
factors. However, in their retrospective analysis of the United States Renal Data System (USRDS), Meier-Kriesche et al. ${ }^{7}$ observed an increased risk of cardiovascular mortality with both ATG and OKT-3 for antibody induction in the initial six months after transplantation (relative risk (RR) 1.27) and beyond this period (RR 1.17). This review is restricted to the drugs commonly used for maintenance therapy in renal transplantation today.

\section{The process of atherosclerosis and arteriosclerosis}

An extensive review of the process of atherosclerosis is beyond the scope of this article. Interesting reviews in this field can be found elsewhere ${ }^{8-11}$.

Atherosclerosis is a multi-factorial disease. The initial step in the process is endothelial injury, presently referred to as "endothelial dysfunction". Endothelial dysfunction is characterized by an imbalance between relaxing and contracting factors, between anti- and pro-coagulant mediators and between growth inhibiting and proliferating factors ${ }^{12}$. Differences in blood flow patterns, laminar flow versus turbulent flow, result in different so-called shear stresses on the endothelium. Early atherosclerotic lesions correlate with regions of turbulent blood flow near branch points or bifurcations ${ }^{11,13}$. Under normal circumstances, the endothelium can react to stressors by generating vasodilating compounds such as prostacyclin and nitric oxide $(\mathrm{NO})^{14}$, the latter being the more important of the $\mathrm{wwo}^{15}$. It is generated from L-arginine by stimulation of endothelial NO-synthase (eNOS) ${ }^{16}$. NO diffuses into the vascular smooth muscle cells, where it stimulates the enzyme guanylate-cyclase. Consequently, the intracellular concentration of cyclic guanylate-monophosphate (cGMP) is raised, resulting in a reduction of intracellular calcium and relaxation of the smooth muscle cells ${ }^{17}$. Because of the disturbed endothelium, the permeability of the endothelium for atherogenic lipid particles, especially low density lipoprotein (LDL), increases and lipid particles accumulate in the vessel wall ${ }^{8}$. The lipid particles undergo metabolic processes, most notably oxidation ${ }^{10}$. This and also the direct injury to the endothelium, stimulate the expression of adhesion molecules, such as intracellular adhesion molecule-1 (ICAM-1) and vascular cell adhesion molecule-1 (VCAM-1) 8,18, $^{8}$ and-1 $)^{19}$. As a result, the influx of inflammatory cells, monocytes and T-cells ${ }^{20,21}$, is stimulated, and monocytes differentiate in the vessel wall into macrophages, which phagocytose the lipid material and form foam cells.

This inflammation is clinically known as fatty streaks, the initial stage in the process of atherosclerosis, which is already present in children and adolescents ${ }^{22,23}$. Oxidized LDL, in itself, facilitates the progression of atherosclerosis by being chemotactic for monocytes ${ }^{24}$ and T-cells ${ }^{25}$ and by impairing the mobility of macrophages ${ }^{24}$. Moreover, oxidized LDL is immunogenic and induces the production of antibodies 26,27 and the formation of immune-complexes, which 
facilitate the phagocytoses of LDL by macrophages 28,29 . The endothelium expresses pro-coagulant, rather than anticoagulant, properties, and forms vasoactive molecules, cytokines and growth factors. The migration and proliferation of vascular smooth muscle cells is stimulated by these growth factors. At that point, the intermediate stage is reached, which is characterized not only by inflammation, but also by proliferation ${ }^{8}$.

As the process continues, the arterial wall thickens. First, the arterial wall can compensate for narrowing the lumen by gradual dilation, a phenomenon called remodelling ${ }^{30}$. Subsequently, increased numbers of macrophages and lymphocytes enter the lesions and proliferate within; activation of these cells leads to the release of hydrolytic enzymes, cytokines, chemokines, and growth factors. Further damage is thus induced and, in the end, focal necrosis and fibrosis disturb the normal vessel wall. This is called an advanced or complicated or mature lesion, characterized by a fibrous cap, consisting of extracellular matrix, smooth muscle cells, and collagen, overlying a core of lipid material and necrotic tissue ${ }^{10}$. At some point in the process, the plaque compromises the vessel lumen and may rupture through the endothelium and promote thrombosis.

Two growth patterns of atherosclerotic plaques have been recognized. The first develops in small and medium sized lesions ( $<40 \%$ diameter reduction) and is characterized by slow and continuous plaque extension, in which the diameter of the vessel is relatively spared. This type of atherosclerotic lesion is correlated to a constant and cumulative burden of the known risk factors. A second type of growth pattern is characterized by occasional acceleration as a result of plaque fissuring, thrombosis, and fibrous organisation of the thrombus. This growth pattern becomes more prominent in advanced lesion sites $(>40 \%$ diameter reduction) and is characterized by pro-coagulant risk factors and is dependent on peak levels of risk factors rather than on the cumulative burden ${ }^{31}$.

A different mechanism of vascular damage is referred to as arteriosclerosis. In contrast to atherosclerosis, which is characterized by inflammation, arteriosclerosis is primarily a degenerative disorder. It is characterized by diffuse fibro-elastic intima thickening, an increase in medial ground substance and collagen, and fragmentation of elastic lamellae with secondary fibrosis and calcification of the media. Unlike atherosclerosis, which occurs mostly in arteries with high shear stresses, arteriosclerosis is generalized throughout the aorta and major arteries ${ }^{32}$. Despite their differences, the two arterial disorders frequently coexist. Atherosclerosis can be visualized by measuring the intima-media thickness (IMT) with ultrasound, usually in the common carotid artery. This IMT shows a clear correlation with cardiovascular endpoints ${ }^{33}$. Using ultrasound, arteriosclerosis can also be visualized by a diminished distensibility or compliance, or by increased stiffening of the artery. An increase in arterial stiffening is associated with an increase in systolic blood pressure and with an increase in pulse pressure. It leads to an increased velocity of the pulse wave over the arteries. This so-called pulse 
wave velocity is highly correlated with cardiovascular and overall mortality 34,35 . The structural abnormalities in the arteries parallel alterations in the left ventricle that result in left ventricle hypertrophy $(\mathrm{LVH})^{36}$. Both disorders are the result of adaptation to the mechanical shear stress of pressure and volume overload ${ }^{37}$. In renal insufficiency, disturbances of the calcium-phosphate metabolism and hyperparathyroidism are contributory factors.

The arterial system has two distinct and separate functions. The first is to deliver blood to the tissues with minimal loss of mean pressure, the so-called conduit function. This function is mostly impaired by atherosclerotic narrowing of the arteries. Secondly, the arteries have to smooth the pulsations resulting from the cardiac ejection and to transform this pulsatile flow into an almost steady flow through the organs, the so-called cushion function ${ }^{32}$. The latter function is predominantly impaired by arteriosclerosis ${ }^{38}$.

Endothelial function is influenced by all of the classical risk factors known from epidemiological studies, that is, hypertension ${ }^{39,40}$, hyperlipidemia ${ }^{41}$, diabetes mellitus $^{42}$, smoking ${ }^{43}$, renal failure ${ }^{44-46}$, and hyperhomocysteinemia ${ }^{47,48}$. Hypertension is thought to impair endothelial function by increasing shear stresses to the endothelium. It impairs the steady laminar flow that up-regulates vasoprotective genes such as eNOS, cyclo-oxygenase 2 (COX-2), and superoxide dismutase $(\mathrm{SOD})^{11}$. Furthermore, cardiovascular risk factors induce the formation of toxic radicals like superoxide $\left(\mathrm{O}_{2}^{--}\right)$. The latter inactivates $\mathrm{NO}$ by the formation of peroxynitrite (ONOO-). Peroxynitrite is a potent oxidant and is capable of inducing lipid peroxidation ${ }^{49}$. The burden of the formation of toxic radicals and its effect on the endothelium and vasculature is indicated in the term "oxidative stress". Superoxide can be neutralized by SOD. SOD thus protects the formation of $\mathrm{NO}^{10,14}$. Homocysteine may induce endothelial dysfunction by accumulation of asymmetric dimethylarginine (ADMA). ADMA is an analogue of the NOprecursor L-arginine and inhibits the enzyme NOS. A possible interaction of homocystein with the functional sulfhydryl moiety of the enzyme dimethylarginine dimethylaminohydrolase (DDAH), which metabolises ADMA, may be responsible for the accumulation of $\mathrm{ADMA}^{48}$. Reduced homocysteine, which has a free sulfhydryl group, is the most deleterious form of homocysteine for the vascular function ${ }^{50}$.

Endothelial function can be tested by stimulating eNOS in two ways. First, shear stress on the endothelium can be enhanced by increasing blood flow. This is usually performed in the forearm after releasing a period of blockade of the blood flow. The result is flow-mediated vasodilation. Secondly, chemical substances can be infused that stimulate endothelial receptors. The most common of these substances is acetylcholine. 


\section{Cardiovascular risk and renal transplantation}

Epidemiological studies have helped to establish several independent risk factors for the development of cardiovascular disease. One of the pivotal studies was the Framingham Heart Study ${ }^{51}$. To date, prospective trials, estimating the cardiovascular risk based on the known population-based risk factors in the transplant population have not been performed; however, it is highly unlikely that these risk factors would have less impact after transplantation. On the contrary, the incidence of cardiovascular disease in the renal transplant population is more than four times higher than in the general population ${ }^{52}$. Extrapolation of the risk associated with diabetes, smoking, and increasing age, as established in the general population, will underestimate these risks in the renal transplant population ${ }^{53}$. An additional risk factor for cardiovascular disease after transplantation is pre-existing disease ${ }^{52,54,55}$. Renal transplant patients have all gone through the stage of endstage renal failure, and cardiovascular mortality in this population is 10-20 times higher than in the general population ${ }^{56}$. Some of the known risk factors are influenced by adverse effects of immunosuppressive therapy. In this review, all known risk factors are discussed, with special attention to the effects that the different immunosuppressive agents have on them.

\section{Firm cardiovascular endpoints}

Prospective studies evaluating the effect of the different immunosuppressive drugs on firm cardiovascular endpoints such as (cardiovascular) mortality, ischemic heart disease, and stroke are scarce. Hollander et al. ${ }^{57}$ reported an $8 \%$ higher incidence of cardiovascular death eight years after transplantation in the patients treated with CsA and corticosteroids than in patients who were converted to AZA and corticosteroids three months after transplantation. Recently, the 15-year follow-up data of the same study were published, revealing an incidence of cardiovascular mortality and an incidence of at least one cardiovascular event that were quite comparable between the groups ${ }^{58}$. In the initial period after transplantation, TAC posed an increased risk of angina pectoris compared with CsA, but the overall incidence of cardiovascular disorders did not differ between both drugs ${ }^{59,60}$. Recently, in a retrospective analysis of the USRDS database, Schnitzler et al. observed a $21-24 \%$ reduction in the risk of death with a functioning graft with the use of MMF compared with AZA three years after transplantation ${ }^{61}$.

Since there is little evidence thus far about the effects of modern immunosuppressive drugs on firm cardiovascular endpoints, it is important to determine the effects of these drugs on the known cardiovascular risk factors as surrogate endpoints in order to determine what strategy to use. 


\section{The effect of immunosuppression on cardiovascular risk factors}

\section{Endothelial function}

Recently, it has been shown that endothelial function improves after renal transplantation compared with hemodialysis ${ }^{62,63}$.

Studies evaluating the effect of corticosteroids on endothelial function in renal transplant recipients have never been performed. However, it was recently shown that corticosteroids impair endothelial function by increasing reactive oxygen species such as superoxide ${ }^{64}$.

Renal transplant recipients treated with CsA had a significant impairment of endothelium-dependent vasodilation compared with AZA-treated patients. The latter group had results comparable with those of controls ${ }^{65}$. However, after conversion from CsA to AZA, no improvement was observed in hypertensive CsA-treated recipients, from whom all anti-hypertensive medication was withdrawn at least three days before the measurements were taken ${ }^{66}$. Since hypertension also impairs endothelial-dependent vasodilation ${ }^{39,40}$, the persistent hypertension may be responsible for the lack of improvement in this study. The mechanism by which CsA impairs endothelial function is probably superoxide production mediated by endothelin (ET) $-1{ }^{67}$.

Studies evaluating the effect of TAC on endothelial function are scarce. One study in a relatively small group of patients showed impaired endothelium-dependent vasodilation in TAC-treated renal transplant recipients compared with healthy controls. The results obtained with TAC were similar to those of patients treated with $\mathrm{CsA}^{68}$. Recently, a second cross-sectional study showed that endothelial function was better preserved with TAC than with CsA ${ }^{69}$.

No studies evaluating RAPA or MMF on endothelial function have yet been published.

In an in vitro capillary tube assay, the effect of the different immunosuppressive drugs was tested on endothelial cells. CsA showed a pronounced injurious effect on the morphology of the in vitro capillaries. In addition, CsA increased the release of ET-1. Neither effect occurred with TAC. MMF also showed a moderate injurious effect on the capillary morphology, whereas RAPA and dexamethasone had no effect. Except for dexamethasone, all agents increased the release of prostacyclin ${ }^{70}$. Antagonizing ET-1 prevented the injury evoked by $\mathrm{CsA}^{71}$.

\section{Hypertension}

Hypertension was one of the first risk factors of cardiovascular disease to be recognized. The Framingham Heart Study studied the relationship between blood pressure and cardiovascular disease. Compared to patients with a blood pressure $<130 / 85 \mathrm{mmHg}$, the relative risk for cardiovascular disease increased to 1.84 for 
males and to 2.12 for females with a blood pressure $>160 / 100 \mathrm{mmHg}$. Some $28 \%$ of cardiovascular events in males and $29 \%$ in females could be attributed to a blood pressure that exceeded $130 / 85 \mathrm{mmHg}^{72}$. Since these data are derived from middle aged, predominantly Caucasian Americans, evaluation in other populations may reveal some differences, but the outcome will generally be the same. After transplantation, hypertension, although treated, was independently associated with all vascular events; however this was not the case when ischemic heart disease or cerebral vascular disease were analyzed separately ${ }^{52}$. The cardiovascular risk of hypertension in the transplant population seems more or less comparable with the risk in the general population ${ }^{53}$. In addition, hypertension negatively influences graft survival ${ }^{73,74}$. Treatment of hypertension in the general population lowered the risk of cardiovascular disease by $20-30 \%$, depending on the drug used or the outcome parameter: stroke, coronary heart disease, major cardiovascular events, or cardiovascular death. The mean decrease in systolic blood pressure for angiotensin converting enzyme (ACE) inhibitors to achieve this reduction was only $3 \mathrm{mmHg}^{75}$. More intensive treatment improved the cardiovascular outcome ${ }^{75}$. Treatment of mild-to-moderate hypertension also prolonged survival in patients with an increased risk of cardiovascular mortality ${ }^{76}$.

The prevalence of hypertension after renal transplantation is high and is related to the immunosuppressive agents used and the timepoint after transplantation ${ }^{77}$. In patients receiving CsA and corticosteroids, the prevalence ranges between 71\% and $78 \% \%^{78,79}$. As well as the immunosuppressive agents, the presence of the endstage native kidneys and the nature of the original disease, renal allograft dysfunction, body mass index, renal artery stenosis, and essential or pre-existing hypertension are also likely to contribute to the hypertension ${ }^{77,78,80-83}$. In addition, a graft derived from a female, elderly or hypertensive donor, or the use of a rightsided donor kidney are associated with post-transplant hypertension ${ }^{81}$.

Corticosteroids have been known to induce hypertension since Cushing described the glucocorticoid excess syndrome. The underlying mechanism has still not been completely elucidated. It was once thought that corticosteroids raised blood pressure as a result of water and salt retention via an effect on the mineralocorticoid receptor. It has since become clear that blockade of NOformation by inhibition of both inducible and endothelial NOS, inhibition of transmembrane arginine transport, and inhibition of the synthesis of the NOS cofactor $\mathrm{BH}_{4}$ play a prominent role ${ }^{84}$. Corticosteroids are also associated with a disturbed circadian blood pressure pattern ${ }^{85}$. Conventional blood pressurelowering treatment has only a moderate effect in Cushing's syndrome in contrast to inhibition of corticosteroid production ${ }^{86}$. Therefore, hypertension after transplantation can be best controlled by avoiding corticosteroids.

It is unclear whether AZA in itself has an effect on blood pressure since it is always used in combination with corticosteroids or CsA. However, the incidence of hypertension, as defined by the use of antihypertensive drugs, was higher in a 
group treated with CsA, AZA, and corticosteroids than in a group treated with CsA and corticosteroids ${ }^{87}$.

MMF has no effect on the blood pressure. The incidence of hypertension was similar between patients treated with MMF $2 \mathrm{~g}$, MMF $3 \mathrm{~g}$, or AZA in combination with $\mathrm{CsA}$ and corticosteroids ${ }^{88}$. In addition, blood pressure remained unchanged after withdrawal of MMF from a triple regimen with CsA and corticosteroids ${ }^{89}$.

CsA monotherapy induces hypertension to the same extent as corticosteroids in combination with $\mathrm{AZA}^{90}$. The incidence of hypertension with $\mathrm{CsA}$ and corticosteroids has been found to be even higher than with AZA and corticosteroids ${ }^{79,91,92}$. After lowering the dose of $\mathrm{CsA}^{93}$ or withdrawing it after conversion to either $\mathrm{AZA}^{90,94-96}$ or $\mathrm{MMF}^{97}$, blood pressure improves significantly. The withdrawal of corticosteroids or conversion from CsA to AZA results in a similar decrease in blood pressure ${ }^{96}$. The complete withdrawal of CsA from a triple drug regimen with MMF and corticosteroids ${ }^{89}$ or with RAPA and steroids ${ }^{98,99}$ resulted in a mean decrease in blood pressure of $11 / 6 \mathrm{mmHg}$ and $6 / 3 \mathrm{mmHg}$, respectively. The latter figure was diminished because of the use of fewer anti-hypertensive drugs in the withdrawal patients. The mechanism by which CsA induces hypertension is complex and has still not been completely elucidated. CsA stimulates trans-membrane influxes of calcium. This facilitates vascular smooth muscle cell contraction and vasoconstriction. Indeed, calcium channel antagonists appear to inhibit CsA-mediated vasoconstriction ${ }^{100}$. However, enhancement of the production of ET-1 ${ }^{101}$, transforming growth factor (TGF)- $\beta$, and renin ${ }^{102}$, inhibition of $\mathrm{NO}$ production, increased sympathetic nerve activity, augmentation of noradrenaline-induced vasoconstriction, increased sodium reabsorption, which could be the result of intra-renal vasoconstriction and subsequent proximal tubular anomaly, and, finally, hypomagnesemia all play a role in the induction of hypertension by $\mathrm{CsA}^{103}$. CsA also impairs the decrease in nocturnal blood pressure ${ }^{95,104}$, which is restored by conversion to AZA ${ }^{95}$.

TAC can induce vasoconstriction and expresses TGF- $\beta$, ET-1, and renin transcription to a comparable extent as $\mathrm{CsA}^{102}$. In the pivotal trials comparing TAC and CsA in combination with AZA and corticosteroids, the incidence of hypertension was comparable ${ }^{59,60}$. However, in liver transplant recipients, hypertension was less frequent in those treated with TAC, although the differences tended to decrease with time after transplantation ${ }^{105,106}$. In more recent reports, TAC resulted in less renal vasoconstriction ${ }^{107}$ and renal transplant patients treated with TAC and equivalent dosages of corticosteroids needed fewer antihypertensive drugs than patients treated with $\mathrm{CsA}^{107,108}$. Margreiter et al. observed a lower incidence of hypertension with TAC than with CsA as the $\mathrm{Neoral}^{\circledR}$ formulation ${ }^{109}$. After conversion from CsA to TAC because of hyperlipidemia, blood pressure did not improve, but converted patients needed fewer antihypertensive drugs ${ }^{110}$. In a crossover design, Ligtenberg et al. showed that blood pressure decreased after conversion from CsA to TAC and returned to baseline 
after reintroduction of CsA $\mathrm{A}^{111}$. Recently, Klein et al. showed that TAC had no effect on blood pressure and renal hemodynamic parameters in healthy volunteers, whereas CsA raised blood pressure by a mean of $15 \mathrm{mmHg}$ and decreased the glomerular filtration rate (GFR) by a mean of $13 \mathrm{ml} / \mathrm{min}^{112}$. In a prospective randomized conversion trial from CsA to TAC in stable renal transplant recipients, blood pressure dropped by a mean of $5 \mathrm{mmHg}$ after conversion to $\mathrm{TAC}^{113}$. These results were sustained up to two years after the intervention ${ }^{114}$. The improvement in blood pressure after conversion was even remarkably higher in a smaller Spanish study, in which both systolic and diastolic blood pressure improved by a mean of $20 \mathrm{mmHg}$ six months after the intervention ${ }^{115}$.

In the study of Kahan et al., increasing doses of RAPA combined with CsA and corticosteroids did not increase the incidence of hypertension ${ }^{116}$. Compared with CsA, AZA, and corticosteroid triple therapy, the incidence of hypertension was $50 \%$ lower with RAPA, AZA, and corticosteroids ${ }^{117}$. Recently, Gonwa et al. showed that diastolic blood pressure was significantly higher (mean of $3 \mathrm{mmHg}$ ) in patients treated with TAC, RAPA, and corticosteroids than in those treated with TAC, MMF, and corticosteroids in the initial six months after transplantation. Systolic blood pressure was also $4 \mathrm{mmHg}$ higher, but this did not reach significance ${ }^{118}$.

\section{Left ventricular hypertrophy}

LVH is related to hypertension ${ }^{119}$, but it is also recognized as an independent risk factor of cardiovascular morbidity and mortality ${ }^{120,121}$. The prevalence among patients with renal insufficiency is high and is related to age, to the degree of renal insufficiency and anemia ${ }^{119}$. Other risk factors include arteriovenous connections, hypertension, extracellular fluid overload, arterial stiffening, and disturbances of the calcium-phosphate metabolism ${ }^{122}$. LVH is associated with an increased cardiovascular mortality after renal transplantation ${ }^{123}$. LVH often improves up to two years after transplantation, but failure to regress has been associated with older age, persisting hypertension, high pulse pressure in normal-size hearts, and low pulse pressure in dilated hearts ${ }^{124,125}$. Since hypertension is the risk factor that can be modified the most, many aspects of immunosuppressive drugs that account for hypertension will account for LVH as well.

However, since calcineurin activity is enhanced in cardiac hypertrophy ${ }^{126}$, both calcineurin inhibitors, CsA and TAC, may influence LVH. Indeed in different animal models of cardiac hypertrophy, both CsA and TAC induced regression of ventricular mass ${ }^{127-130}$. In contrast, in children, higher TAC trough concentrations were associated with reversible, concentric, hypertrophic cardiomyopathy that could not be accounted for by the concomitant use of corticosteroids ${ }^{131,132}$.

In adult bone marrow transplant recipients treated with TAC or CsA to prevent graft-versus-host disease, left ventricular mass increased slightly, but not 
significantly, with TAC. The increase in left ventricular mass with CsA was higher and reached statistical significance. In addition, significantly more patients developed hypertrophic cardiomyopathy with CsA. A rise in blood pressure with CsA and the concomitant use of corticosteroids may account for the observed difference ${ }^{133}$. In heart transplant recipients, the development of LVH posttransplantation was positively correlated with CsA trough concentrations ${ }^{134}$. In a cross-sectional study, LVH tended to be higher with a CsA-based regimen than with AZA-based immunosuppression in renal transplant patients ${ }^{104}$. The direct influence of RAPA on LVH is unknown at the moment.

Since anemia is also a risk factor for $\mathrm{LVH}, \mathrm{MMF}^{135}$ and $\mathrm{AZA}^{136}$, which impair bone marrow function, may also have an indirect negative effect on left ventricular mass. RAPA also induces anemia. A greater number of patients developed anemia with RAPA than with MMF, both in combination with TAC and corticosteroids ${ }^{118}$. Moreover, the hemoglobin level with RAPA $5 \mathrm{mg}$ was lower than with AZA but similar to AZA with RAPA $2 \mathrm{mg}^{137}$.

Hyperparathyroidism is also associated with LVH. Thus far, no data exist that indicate how immunosuppressive drugs influence the parathyroid hormone. However, because of their nephrotoxicity ${ }^{138}$, both TAC and CsA may have an indirect negative effect.

\section{Hyperlipidemia}

Hypercholesterolemia is another recognized risk factor for cardiovascular disease. Since the main intervention trials with HMG-CoA reductase inhibitors (statins), it has become clear that, in the general population, an increase in cholesterol increases the risk of cardiovascular disease ${ }^{139}$. Some $27 \%$ of all cardiovascular events in males and $34 \%$ in females were attributable to a cholesterol $>5.2 \mathrm{mmol} / \mathrm{l}(200 \mathrm{mg} / \mathrm{dl})$, the cut-off level of the National Cholesterol Education Program (NCEP) in the United States ${ }^{72}$. In the 4S (Scandinavian Simvastatin Survival Study), a reduction of $1 \%$ in total and LDL-cholesterol reduced the risk of major coronary events by $1.9 \%$ and $1.7 \%$, respectively. An increase of $1 \%$ in high-density lipoprotein (HDL)-cholesterol reduced the risk by $0.8 \% \%^{140}$.

Hypercholesterolemia as a risk factor for cardiovascular disease in renal transplant patients has mainly been evaluated in retrospective and cross-sectional studies. The latter have shown an association between hypercholesterolemia and cardiovascular disease ${ }^{52,141}$. In a retrospective analysis, cholesterol was negatively associated with patient survival but the effect diminished with increasing age ${ }^{142}$, while, in another study, a significantly improved survival was only observed in recipients with a pre-transplant cholesterol level of $<5.5 \mathrm{mmol} / \mathrm{1}^{143}$. The risk associated with hypercholesterolemia resembles that in the general population ${ }^{53}$. In a retrospective analysis, intervention with statins reduced all-cause mortality by $24 \%$ in renal transplant recipients when controlling for age, cholesterol level before the start of statins, and transplant year ${ }^{144}$. In addition, hypercholesterolemia 
also negatively influenced graft survival ${ }^{142,145,146}$. Recently, the results of a prospective intervention study, the ALERT (Assessment of Lescol in Renal Transplantation) trial, were published ${ }^{147}$. Intervention with fluvastatin $40 \mathrm{mg}$ in CsA-treated transplant recipients with a baseline total cholesterol between 4 and $9 \mathrm{mmol} / 1$ showed a significant $35 \%$ risk reduction on the composite endpoint of cardiac death and definite non-fatal myocardial infarction after five years of follow-up. However, on the primary endpoint of overall cardiac incidents (cardiac death, non-fatal myocardial infarction, coronary artery bypass grafting, and percutaneous coronary interventions) only a trend towards a $17 \%$ risk reduction was observed. Post-hoc risk factor analysis in the placebo-treated patients revealed a $41 \%$ increase in the risk of the primary endpoint and the composite endpoint of cardiac death and non-fatal myocardial infarction per $1.0 \mathrm{mmol} / \mathrm{l}$ increase in LDL-cholesterol. Remarkably, no influence of the intervention was observed on all cause death or graft survival. In addition, statin therapy did not protect against cerebrovascular accidents ${ }^{147}$. Whether more aggressive lipid-lowering therapy would have an effect on cerebrovascular incidents similar to the results in the ASCOT (Anglo-Scandinavian Cardiac Outcomes Trial) ${ }^{148}$, in which the patient population consisted of hypertensive patients with an average or lower than average cholesterol, is still unclear.

The prevalence of hyperlipidemia after transplantation is high and about $60 \%$ of all transplant patients have cholesterol levels in the high-risk category $(>6.3 \mathrm{mmol} / \mathrm{l})$, according to the guidelines of the $\mathrm{NCEP}^{77}$. Not only the immunosuppression but also other drugs, such as $\beta$-adrenoceptor antagonists, and genetic and environmental factors, such as diet, contribute to hypercholesterolemia ${ }^{149}$.

Corticosteroids increase both total cholesterol and LDL-cholesterol ${ }^{150-152}$, but they also increase HDL-cholesterol ${ }^{150,151,153}$. Corticosteroids are also positively correlated with serum triglycerides ${ }^{154-157}$.

Corticosteroids increase the hepatic synthesis of very low density lipoprotein (VLDL) by enhancing the activity of the rate-limiting enzymes acetyl-co-enzyme A carboxylase and free fatty acid (FFA) synthetase ${ }^{158}$. The effect on the first enzyme results from a concomitant hyperinsulinemia ${ }^{158}$. Hyperinsulinemia may be an important factor in corticosteroid-induced hyperlipidemia since the correlation between serum triglycerides and plasma insulin levels is highly significant in renal allograft recipients ${ }^{154}$. Corticosteroid-induced hyperinsulinemia may increase the uptake of FFAs in the liver, the main substrate for the synthesis of VLDL, but this mechanism may be of minor importance ${ }^{159}$. Insulin resistance results in a decreased action of peripheral lipoprotein lipase (LPL), which is responsible for the clearance of triglycerides ${ }^{160}$. Insulin resistance may also promote the oxidation of LDL ${ }^{161}$. The conversion from VLDL to intermediate density lipoprotein (IDL) and subsequently LDL, which is unaffected by corticosteroids, may account for the hypercholesterolemia ${ }^{162}$. The uptake and degradation of LDL is reduced by 
corticosteroids $^{163}$, and this results from a decrease in LDL-receptor synthesis and subsequent expression ${ }^{164,165}$. The increase in HDL probably results from the depression of plasma cholesteryl-ester transfer protein (CETP) ${ }^{166-168}$.

CsA also induces hyperlipidemia in a dose-dependent way ${ }^{169}$. In patients with amyotrofic lateral sclerosis, without confounding co-medication or impaired renal function, CsA enhanced total cholesterol by $21 \%$, LDL-cholesterol by $31 \%$, and, although not significantly, triglycerides by $17 \% 0^{170}$. After renal transplantation, total and LDL-cholesterol increased in patients treated with CsA compared with in those receiving $\mathrm{AZA}^{171}$. In addition, CsA was negatively correlated with HDLcholesterol levels ${ }^{169}$. Hyperlipidemia was reversible and total cholesterol decreased by $0.5-1 \mathrm{mmol} / 1$ after conversion from CsA to $\mathrm{AZA}^{94,172,173}$, after conversion from CsA to $\mathrm{MMF}^{97}$, or after withdrawal of $\mathrm{CsA}^{174}$. The decrease in total cholesterol was the result of a decrease in LDL-cholesterol172,173. However, subfraction determination of the lipoproteins revealed a decrease in IDL-cholesterol in the study with $\mathrm{MMF}^{97}$. HDL-cholesterol did not change after conversion to $\mathrm{AZA}^{172,173}$, but it decreased after conversion to $\mathrm{MMF}^{97}$. CsA augmented the hyperlipidemia induced by corticosteroids ${ }^{175}$. Triglycerides were higher in patients treated with CsA than in patients treated with AZA and corticosteroids or MMF and corticosteroids ${ }^{94,97,172,176,177}$. In rats treated with CsA, plasma levels of triglycerides, VLDL-triglycerides, total cholesterol, and LDL-cholesterol were increased, whereas HDL-cholesterol was decreased ${ }^{175,178,179}$.

CsA interferes with lipid metabolism in several ways. It decreases the activity of LPL, which is probably the basis for the observed hypertriglyceridemia ${ }^{175,178}$. CsA impairs the clearance of LDL ${ }^{179}$. In vitro studies have shown that this effect of CsA is mediated by a decreased LDL-receptor synthesis in a way similar to corticosteroids ${ }^{164,180}$. Both drugs act synergistically in this field ${ }^{164}$. CsA blocks the 27-hydroxylation of cholesterol in mitochondria by a mitochondrial cytochrome P450 enzyme system, thereby enhancing the intracellular concentration of free cholesterol and subsequent down-regulation of LDL-receptor activity ${ }^{181}$. However, in vivo, in rats treated with CsA, no reduction in LDL-receptor mass was observed $^{178}$. CsA also reduces the activity of $7 \alpha$-hydroxylase, the enzyme that is the rate-limiting step in the conversion of cholesterol into bile acid, which is the principal pathway of cholesterol catabolism ${ }^{178}$. Hyperlipidemia by CsA is not mediated by interference with the rate-limiting enzyme of the cholesterol synthesis, HMG-coA reductase ${ }^{178}$. Nor does the presence of CsA in the LDLparticle interfere with the binding of LDL to its receptor and the subsequent uptake ${ }^{179,180}$.

In addition to its effects on total cholesterol and LDL-cholesterol, CsA enhances lipoprotein(a) (lp(a)), as evidenced by CsA-treated patients having significantly higher levels than patients converted to $\mathrm{AZA}^{153}$. Lp(a) is a lipid particle comparable to LDL, but is composed of different lipoproteins. Other studies have not been able to confirm this observation ${ }^{97,182}$. 
CsA increases the oxidation of $\mathrm{LDL}^{172,183,184}$, although Devaraj et al. did not observe a direct effect of CsA on LDL oxidation. Thus, the former observation may be the result of a decrease in plasma triglycerides, resulting in a different, increased, and less dense composition of the LDL-particle after CsA withdrawal ${ }^{185}$.

Although at first it was not known that TAC might improve hypertension, in the main US trial comparing TAC to CsA, it became clear that hyperlipidemia, especially hypercholesterolemia, was less frequent in TAC-treated patients ${ }^{59}$. In a later report from the main European trial, both total and LDL-cholesterol were lower in TAC-treated patients than in CsA-treated patients at one year. Cholesterol levels with TAC were no different than baseline levels prior to transplantation, whereas there was an increase in the CsA group ${ }^{186}$. Triglycerides were comparable in TAC- versus CsA-treated patients ${ }^{187}$. Conversion from CsA to TAC because of hyperlipidemia resulted in a $16 \%$ decrease in total cholesterol and a $25 \%$ decrease in LDL-cholesterol. Triglycerides improved slightly, but not significantly, after conversion, whereas HDL-cholesterol did not change ${ }^{188}$. Another group published similar results, with a significant decrease in serum triglycerides after conversion to $\mathrm{TAC}^{110}$. In a randomized, prospective trial evaluating conversion from CsA to TAC in stable transplant patients, total cholesterol improved significantly by a mean of $0.5 \mathrm{mmol} / \mathrm{l}$, LDL-cholesterol by $0.35 \mathrm{mmol} / \mathrm{l}$, and triglycerides by $0.4 \mathrm{mmol} / \mathrm{l}^{113}$. These results were sustained up to two years after conversion ${ }^{114}$. Similar results were reported by a Spanish group $^{115}$.

$\mathrm{Lp}(\mathrm{a})$ was also found to be lower with TAC compared with CsA ${ }^{187}$. However, after conversion from CsA to TAC, $\mathrm{Lp}(\mathrm{a})$ did not change ${ }^{189}$.

Besides the improvement in serum lipid levels, the oxidation of LDL from TACtreated, but not CsA-treated, patients was comparable with that of healthy controls ${ }^{183,184}$. Varghese et al., on the other hand, observed an enhanced susceptibilty to oxidation of LDL, isolated from TAC-treated transplant patients, compared with LDL, isolated from Neoral ${ }^{\circledR}$-treated patients. The fortification of the Neoral ${ }^{\circledR}$ formulation with tocopherol (vitamin E), which has anti-oxidant properties, may account for this remarkable difference ${ }^{10,191}$. Morena et al. observed a similar susceptibility to oxidation of LDL derived from both CsAtreated and TAC-treated patients ${ }^{192}$. However, the best evidence of the difference between TAC and CsA originates from the study of randomized conversion from CsA to TAC. In that study, LDL-oxidation clearly improved after conversion to TAC ${ }^{189}$. The data from the Dutch trial are completely in line with the results of a Spanish conversion study ${ }^{115}$.

It was already clear in phase I and II studies that RAPA raised both total cholesterol and triglycerides to high levels, and the increase appeared to be dosedependent ${ }^{116,193-195}$. When RAPA was added to CsA in a phase III trial, total cholesterol increased by $0.8 \mathrm{mmol} / \mathrm{l}$ (RAPA $2 \mathrm{mg}$ ) to $1.4 \mathrm{mmol} / \mathrm{l}$ (RAPA $5 \mathrm{mg}$ ) 
and triglycerides increased by $1.1 \mathrm{mmol} / 1$ (RAPA $2 \mathrm{mg}$ ) to $2.0 \mathrm{mmol} / 1$ (RAPA $5 \mathrm{mg}$ ), respectively, after six months of therapy, compared with the addition of $\mathrm{AZA}^{137}$. The addition of RAPA $10 \mathrm{mg}$ to CsA and corticosteroids for six weeks in trials focusing on serum lipids raised both total and LDL-cholesterol by $50 \%$ and triglycerides by almost $100 \%$. The effects were fully reversible after discontinuation of RAPA ${ }^{196,197}$. Rapa is a stronger inducer of hyperlipidemia than CsA, as shown in phase III trials. Six months post-transplantation, total cholesterol and triglycerides were $1.5 \mathrm{mmol} / 1$ and $0.8 \mathrm{mmol} / 1$, respectively, higher in patients treated with RAPA (target trough concentration $15 \mathrm{ng} / \mathrm{ml}$ ) in combination with MMF and corticosteroids than in patients treated with CsA (target trough concentration of CsA 100-200 ng/ml), MMF and corticosteroids ${ }^{198}$. At RAPA trough concentrations around $30 \mathrm{ng} / \mathrm{ml}$ two months posttransplantation, the difference with CsA (trough levels 200-400 ng/ml), in combination with AZA and corticosteroids, was maximally $2.8 \mathrm{mmol} / \mathrm{l}$ for total cholesterol and $3.2 \mathrm{mmol} / 1$ for triglycerides, respectively ${ }^{117}$. In combination with TAC and corticosteroids, even low dosages of RAPA $(0.5 \mathrm{mg})$ increased the incidence of hypercholesterolemia by $15 \%$, although the effects were less prominent than the combination with $\mathrm{CsA}$ and corticosteroids. The increase in total cholesterol ranged between $0.4 \mathrm{mmol} / \mathrm{l}(\mathrm{RAPA} 0.5 \mathrm{mg}$ ) and $1.0 \mathrm{mmol} / \mathrm{l}$ (RAPA $2 \mathrm{mg})^{199}$. At six months post-transplantation, total cholesterol, LDLcholesterol, and triglycerides were significantly higher $(0.67 \mathrm{mmol} / 1,0.34 \mathrm{mmol} / \mathrm{l}$, and $0.59 \mathrm{mmol} / \mathrm{l}$, respectively) with RAPA (target trough concentrations 4$12 \mathrm{ng} / \mathrm{ml}$ ) in combination with TAC and corticosteroids than in the combination of MMF, TAC and corticosteroids ${ }^{118}$. The mechanism by which RAPA induces changes in lipid levels has still not been completely elucidated. In studies in transplant recipients with prominent hyperlipidemia while receiving RAPA therapy, the VLDL, IDL, and LDL-fractions and accompanying apoproteins were increased. Initially, it was shown that the catabolism of these lipoproteins was reduced ${ }^{200}$. An additional study showed that the FFA pool was expanded by RAPA and that the hepatic production of triglycerides and secretion of VLDL was increased ${ }^{196,197}$. Rapa enhanced the action of hormone sensitive lipase (HSL) and may have inhibited LPL, although the effects on the latter enzyme were not unequivocal ${ }^{196,197,200}$. The effects of RAPA on lipid metabolism appear to be quite opposite to the effects induced by insulin. Therefore, interference by RAPA of an insulin-dependent signaling pathway has been suggested as the mechanism underlying the disturbance of lipid metabolism ${ }^{196,197}$. Thus far, no data have been published about the effect of RAPA on Lp(a) or on the oxidation of LDL.

MMF has no effect on serum lipids. Cholesterol and triglycerides were unchanged after discontinuation of MMF from the combination of TAC, MMF, and corticosteroids $^{152}$, and total cholesterol was similar between patients treated with TAC, MMF, and corticosteroids and patients treated with TAC and corticosteroids $^{201}$. The effects of AZA on cholesterol are similar to those of MMF. 
Total cholesterol was comparable between patients treated with TAC, AZA, and corticosteroids and patients treated with only TAC and corticosteroids ${ }^{136}$. Total and LDL-cholesterol, and triglycerides were all similar between patients treated with CsA, AZA, and corticosteroids and patients who had AZA withdrawn from the same regimen ${ }^{202}$. There are no published data about the effect of either drug on $\mathrm{Lp}(\mathrm{a})$ or oxidized LDL to date.

\section{Diabetes mellitus}

Diabetes mellitus is also strongly associated with an increased cardiovascular mortality and morbidity. In the Framingham Heart Study, diabetes increased the risk of coronary heart disease by $53 \%$ in men and as much as $82 \%$ in women ${ }^{72}$. In renal transplantation, the risk is dramatically higher in both males (3-fold) and females (5-fold) $)^{53}$. Furthermore, the development of diabetes is associated with an impaired graft survival ${ }^{203,204}$.

Corticosteroids disturb glucose tolerance by enhancing insulin resistance ${ }^{205}$ and play a significant role in the development of post-transplant diabetes mellitus (PTDM) ${ }^{206,207}$. Withdrawal of corticosteroids reduces the incidence of PTDM and improves glycemic control ${ }^{208}$. Early corticosteroid withdrawal after transplantation may be an important factor in the prevention of PTDM ${ }^{209}$.

CsA also has an effect on glucose metabolism. In liver transplant recipients treated with CsA, glucose tolerance was impaired compared with control patients (hepatitis C-negative patients with chronic liver disease) ${ }^{210}$. Renal transplant patients receiving CsA and corticosteroids had a higher incidence of PTDM than patients treated with AZA and corticosteroids ${ }^{204,211}$, even when corticosteroid dosages were higher ${ }^{211}$. Reducing the dose of CsA reversed the diabetes in the majority of affected patients ${ }^{211}$. Glucose tolerance also improved after conversion from CsA to $\mathrm{AZA}^{173}$. The source of the diabetic effect of CsA is an impairment in pancreatic $\beta$-cell secretory function ${ }^{210,212}$. In addition, glucagon production by the $\alpha$-cells is enhanced ${ }^{210}$. In in vitro experiments, CsA dose-dependently inhibited insulin secretion ${ }^{213,214}$. However, no effect on glucagon secretion was observed ${ }^{214}$. In the initial report comparing CsA and TAC, it was mentioned that the incidence of PTDM was comparable between the drugs ${ }^{215}$. However, the main trials showed a significantly higher incidence with TAC 59,60 ; with an incidence of PTDM of around $20 \% 0^{59,216}$ (versus an incidence of $4 \%$ with CsA in the US trial ${ }^{59}$ ). In a majority of patients, PTDM is reversible after reducing the dose of TAC and withdrawing corticosteroids ${ }^{216,217}$. Subsequently, after the first six months posttransplantation, the incidence of PTDM decreases to $5.7 \%$ with tacrolimus ${ }^{218}$. In particular, patients who already had subclinical impairment of glucose tolerance were at risk of developing PTDM with TAC ${ }^{219}$. Despite the higher incidence of PTDM with TAC, maintenance levels of both calcineurin inhibitors showed no differences with regard to glucose metabolism ${ }^{210,220}$. Cumulative toxicity was absent ${ }^{220}$. TAC decreased insulin secretion by pancreatic $\beta$-cells in a dose- 
dependent way210,219,221,222, and glucagon expression was also enhanced ${ }^{210}$. However, others observed an increase in insulin resistance 223 . In in vitro experiments, TAC: (i) dose-dependently and completely reversibly inhibited insulin gene transcription starting after 24 hours; (ii) had no acute effects on insulin secretion; and (iii) did not affect glucose uptake by insulin. Since calcineurin and FK-binding protein (FKBP)-12 are also present in $\beta$-cell lines, the inhibition of insulin secretion by TAC probably occurs along the same pathway as the interference of IL-2 production ${ }^{224,225}$. Although in different concentrations, both calcineurin and FKBP-12 are found in $\alpha$-cells ${ }^{225}$, which may explain the upregulation of glucagon ${ }^{210}$. The stronger potency of TAC, compared with CsA, to block calcineurin ${ }^{226}$ may account for the higher incidence of PTDM observed in TAC-treated patients than in CsA-treated patients in the initial period after transplantation $59,60,109$.

RAPA has no known effect on glucose metabolism. The incidence of PTDM was unchanged with the addition of RAPA to either CsA-116 or TAC-based regimens ${ }^{118}$.

\section{Renal function}

Impaired renal function is strongly associated with an increased risk of cardiovascular death, also after renal transplantation. Cardiovascular mortality 10 years post-transplantation increased from $4 \%$ for recipients with a normal serum creatinine $(<115 \mu \mathrm{mol} / \mathrm{l})$ at one year post-transplantation to $9 \%$ for recipients with a serum creatinine $>230 \mu \mathrm{mol} / \mathrm{1}^{227}$. Moreover, recipients with a compromised transplant function at one year post-transplantation (GFR $44.8 \mathrm{ml} / \mathrm{min} / 1.73 \mathrm{~m}^{2}$ ) had an increased risk of both acute coronary syndromes (adjusted hazard ratio (HR): 2.16) and congestive heart failure (adjusted HR: 2.95)228.

Both calcineurin inhibitors are nephrotoxic agents. This nephrotoxicity can be divided into two parts: (i) an initially reversibly dose-dependent renal vasoconstriction $^{229}$; and (ii) in the chronic progressive changes. Histologically, no differences can be shown between the two drugs. Both induce tubulopathy, characterized by vacuolation of epithelial cytoplasm with or without microcalcifications, arteriolopathy, and glomerulopathy. Initially, arterioles show prominent vacuolation of smooth muscle cells. Later on, these smooth muscle cells may undergo necrosis and necrotic cells are replaced by proteinaceous material. Glomerular lesions evolve to focal segmental glomerulosclerosis. Finally, calcineurin-induced nephrotoxicity results in tubular atrophy and interstitial fibrosis ${ }^{138}$.

The vasoconstriction is mediated by ET-1 in the afferent arteriole 230,231 . CsA augments the action of ET-converting enzyme and augments the production of ET-1 by endothelial cells mediated by the enhanced production of TGF- $\beta^{232}$. ET-1 also plays a role in the development of vasculopathy ${ }^{233-235}$. TGF- $\beta$ is a potent inducer of fibrosis formation, and CsA not only upregulates TGF- $\beta 1$ 
expression but also its receptors in rat mesangial cells ${ }^{236}$. The chronic tubulointerstitial fibrosis induced by CsA is thought to be mediated by TGF- $\beta^{237-239}$ and by inducing apoptosis in renal tubular cells ${ }^{240}$. However, recently, no toxic effect of CsA on tubular cells could be demonstrated in vitro and the in vivo observed results may be induced by renal vasoconstriction ${ }^{241}$. CsA may accumulate in the kidney ${ }^{242}$, enhancing its nephrotoxicity. The combination of CsA with RAPA results in a serious interaction, leading to accumulation of CsA in the kidney and thus aggravating its nephrotoxicity ${ }^{243}$. This interaction has not been observed between RAPA and TAC ${ }^{244}$, but there are indications that this combination may result in an increased risk of delayed graft function ${ }^{245}$. Moreover, patients treated with TAC and MMF had a better renal function than patients treated with TAC and RAPA ${ }^{118}$.

Although both calcineurin blockers are histologically equally nephrotoxic, more and more evidence has arisen that TAC may be less nephrotoxic. There is less renal vasoconstriction in healthy volunteers with TAC than with CsA ${ }^{112}$. Gene expression of fibrosis formation is lower in both TAC-treated mice ${ }^{246}$ and humans ${ }^{247}$ than with CsA treatment. Furthermore, TGF- $\beta$ expression in kidney biopsies is lower in those taken from TAC recipients than CsA recipients ${ }^{248}$. FKBP-12 binds to the TGF- $\beta-1$ receptor ${ }^{249,250}$, and it also acts as an anchor of another cytoplasmic protein to that receptor, probably calcineurin. The complex of FKBP-12 and calcineurin prevents the receptor from being activated ${ }^{249}$. Macrolide derivatives of TAC, which can bind to FKBP-12, but have no effect on calcineurin, can remove the FKBP-12/calcineurin complex from the TGF- $\beta-1$ receptor and enhance the activation of the TGF- $\beta$ receptor ${ }^{249}$. However, TAC, which binds to FKBP-12 and inactivates calcineurin, appears to exhibit growth inhibition mediated by TGF- $\beta$ 249. The downstream blockade of calcineurin and subsequent gene transcription is, therefore, essential for interruption of the effects of TGF- $\beta$. In contrast, CsA induces the expression of TGF- $\beta-1$ receptors ${ }^{236}$. In serum, both TAC and CsA enhance the production of TGF- $\beta 251$. Besides their different effects on TGF- $\beta$ expression, CsA and TAC may also have different effects on ET-1 secretion. Whereas CsA enhanced the production, TAC appeared to have no effect in a cultured renal cell line ${ }^{252}$ or in a capillary tube assay ${ }^{70}$. However, another study did not find any difference in ET mRNA expression in renal biopsies from patients receiving either CsA or $\mathrm{TAC}^{102}$. Recently, it has prospectively been shown that fibrosis formation was indeed less in TAC-treated recipients than in CsA-treated patients 253,254 .

Clinically, the main prospective trials observed a comparable graft function both with CsA-Sandimmune ${ }^{\circledR 59,60}$ and with CsA-Neoral ${ }^{\circledR 109}$, despite a significant reduction in the incidence of acute rejection. However, in their retrospective analysis of the USRDS, Gjertson et al. ${ }^{255}$ observed that in the long-term TACtreated patients had a better graft survival. In the past year, the five-year follow-up data of the main trials comparing TAC with CsA-Sandimmune ${ }^{\circledR}$ were published. 
In the US trial, graft function was comparable between the two groups on an intention-to-treat analysis ${ }^{256}$. However, a substantial proportion of the CsA-treated patients were converted to TAC, predominantly as a result of lack of efficacy. After censoring for these converted patients, it was shown that patients treated with TAC had a better graft survival. Moreover, creatinine clearance was significantly higher in the TAC-treated patients. The five-year results of the European trial showed a comparable graft survival, but the incidence of chronic rejection was significantly lower in the TAC-group and the projected graft half-life was higher in the TAC-treated patients ${ }^{257}$. Remarkably, not only patients who received TAC from transplantation onwards may profit from a better renal function with TAC. Serum creatinine decreased six months after conversion from CsA to TAC in patients with a stable graft function more than a median of five years after a successful transplantation ${ }^{113}$. The two-year follow-up data of that trial showed a stable transplant function in the conversion group in contrast to a gradual decline in the group that stayed on CsA ${ }^{114}$. Kaplan et al. ${ }^{258}$ compared kidneys derived from the same donor, where one kidney was allocated to a recipient with initial treatment with microemulsion CsA (formulation not specified) and the other kidney to a recipient with initial treatment with TAC. Recipients with TAC-based immunosuppression showed lower creatinine values over the whole five-year study period. However, five-year graft survival and the rate of decline in renal function were similar in both groups ${ }^{258}$.

\section{Homocysteine}

Hyperhomocysteinemia is recognized as a cardiovascular risk factor by the extreme juvenile atherosclerosis observed in patients with classically homozygote homocystinuria ${ }^{259}$. These patients lack one of the enzymes involved in homocysteine metabolism: methylene-tetrahydrofolate reductase (MTHFR), cystathione $\beta$-synthase, or methionine synthase. They have high fasting homocysteine levels of $>50 \mu \mathrm{mol} / 1$. The incidence of this disease is rare, approximately 1 per 100.000 population ${ }^{260,261}$. More subtle elevations of the homocysteine level of $>15 \mu \mathrm{mol} / 1$, which is proposed as the cut-off level ${ }^{262}$, are more frequent and are present in about $25 \%$ of patients with cardiovascular disease versus $5-10 \%$ of control populations ${ }^{263}$. These patients do not lack one of the enzymes but rather these enzymes have mutations. The most common is the C677T-mutation in the MTHFR gene. Some 10-13\% of Caucasian populations are homozygous for this mutation ${ }^{261,264}$. The function of these mutated enzymes is decreased. The vitamin status, especially folate, of the person determines whether they will have hyperhomocysteinemia ${ }^{265,266}$. Homocysteine levels are higher in men than in women and increase with age 267 . More and more evidence has arisen that these less dramatic increases in serum homocysteine are also associated with an increased risk of cardiovascular disease. A meta-analysis of retrospective studies showed that the odds ratio (OR) of coronary artery disease was 1.6 in men for 
each $5 \mu \mathrm{mol} / \mathrm{l}$ increase in homocysteine level versus 1.8 in women. Some $10 \%$ of the risk of coronary artery disease in the population could be attributed to increased homocysteine levels. The risk of a $5 \mu \mathrm{mol} / 1$ increase in homocysteine was comparable with the risk of a $0.5 \mathrm{mmol} / \mathrm{l}$ increase in cholesterol ${ }^{263}$. Prospective studies revealed lower risks than the case control studies. Metaanalyses of the prospective trials revealed ORs for ischemic heart disease for every $5 \mu \mathrm{mol} / 1$ increase in homocysteine of $1.2^{268}$ and 1.23269 , respectively.

Serum homocysteine levels are dependent on renal function ${ }^{270-273}$. In dialysis patients, the remethylation and the transmethylation of homocysteine are disturbed. The other metabolic route (transsulfuration) is unaffected ${ }^{274}$. In normal kidneys, no significant renal extraction of either total or free homocysteine was demonstrated. Therefore, less renal elimination as the result of the decline in GFR can not explain the higher homocysteine levels in chronic kidney disease ${ }^{275}$. Although homocysteine levels are correlated to the GFR, higher homocysteine levels were not related to the decline in renal function in chronic kidney disease in one study $\mathrm{y}^{276}$.

The prevalence of hyperhomocysteinemia after renal transplantation is high and ranges from $50 \%{ }^{277-280}$ to $80 \% 0^{281,282}$ of the transplant recipients. Homocysteine levels are two- to six-fold higher in renal transplant recipients compared with healthy controls ${ }^{281}$. Compared with the dialysis status, homocysteine levels improve by a mean of $14 \%$ six months after transplantation 283 . In patients with immediate good graft function, homocysteine levels decrease directly after transplantation 284 . After transplantation, homocysteine levels are related to the GFR $^{277,280,282,283,285}$, genetic factors 278,286 , the nutritional status ${ }^{282}$ and age ${ }^{287}$, and are higher in males than in females 280,285 . Hyperhomocysteinemia is also associated with an increased risk of cardiovascular disease after renal transplantation ${ }^{287-289}$, with a RR of 1.06 for each $1 \mu \mathrm{mol} / \mathrm{l}$ increase in plasma homocysteine ${ }^{287}$. However, other studies have shown no such relationship290,291. Despite the relationship between homocysteine and cardiovascular disease and renal function, no negative effect of hyperhomocysteinemia on either patient or graft survival has been demonstrated 292,293 .

A limited number of studies evaluating the effect of immunosuppressive drugs on homocysteine levels have been published in the last decade. Arnadottir et al.285 observed higher homocysteine levels in recipients treated with CsA-based immunosuppression than in patients that were not treated with CsA. These latter patients had homocysteine levels comparable to patients with chronic renal failure with a similar GFR. Despite the higher homocysteine levels, no correlation with CsA trough concentrations was observed ${ }^{285}$. However, in cardiac transplant patients, CsA trough concentrations correlated more to homocysteine levels than serum creatinine ${ }^{294}$. Mor et al. ${ }^{295}$ observed also lower homocysteine levels in patients treated with AZA and corticosteroids compared with those treated with CsA, AZA, and corticosteroids, but the CsA-treated patients had a significantly 
higher serum creatinine. On the other hand, Ducloux et al. ${ }^{296,297}$ showed that after matching for confounding variables, CsA did not increase homocysteine levels and that homocysteine was more related to transplant function and folate status. In addition, in an in vitro experiment, Ignatescu et al. ${ }^{298}$ showed that CsA had no influence on homocysteine formation in proximal renal tubular cells.

Several studies evaluated homocysteine levels between CsA- and TAC-treated patients. All studies observed similar homocysteine levels in both CsA- and TACtreated recipients $281,295,299$. Mehra et al.300 showed the same results in heart transplant recipients. After randomized conversion from CsA to TAC, Artz et al. 189 also observed no improvement in homocysteine levels. Similarly to CsA, TAC showed no effect on homocysteine formation in an in vitro experiment with proximal renal tubular cells ${ }^{301}$. On the other hand, Quiroga et al. ${ }^{302}$ observed significantly lower homocysteine levels 12 months post-transplantation in TACtreated patients than in CsA-treated patients, but data on renal function were not mentioned.

In contrast to CsA and TAC, MMF decreased homocysteine formation in the same in vitro experiment ${ }^{301}$. In male transplant recipients, homocysteine levels were $1 \mu \mathrm{mol} / 1$ lower in patients treated with CsA, MMF, and corticosteroids than in patients treated with CsA, AZA, and corticosteroids. However, in females, no differences were observed ${ }^{301}$.

There are currently no data about the effect of corticosteroids or RAPA on homocysteine levels.

\section{Thrombosis and fibrinolysis}

In the last decade, it has become clear that acute thrombus formation is responsible for acute coronary syndromes. Therefore, fibrinolytic therapy has become an important part of the treatment of myocardial infarction. Several proteins involved in coagulation or fibrinolysis such as tissue factor (factor VII), fibrinogen, von Willebrand factor (vWF), tissue-type plasminogen activator (tPA), and plasminogen activator inhibitor type 1 (PAI-1), have been related to the development of coronary events ${ }^{303-308}$. The increased risk of coronary events associated with tPA reflects increased tPA-PAI-1 complexes and results in a reduced fibrinolytic capacity 304 . The relative risk of coronary events of the highest quintiles versus the lowest quintiles was 2.89 for fibrinogen, 1.85 for vWF, and 2.10 for tPA304. PAI-1 levels are associated with insulin levels and insulin resistance ${ }^{309,310 .}$.

PAI-1 has also shown to be an independent risk factor of coronary artery disease in hemodialysis patients ${ }^{311}$, and reduced fibrinolytic capacity has been related to the development of coronary artery disease in heart transplant recipients ${ }^{312}$. A reduced fibrinolytic potential was demonstrated in nearly $70 \%$ in corticosteroidtreated transplant recipients ${ }^{313}$. Moreover, renal transplant recipients have high levels (above a mean of $200 \%$ ) of both vWF and factor VIII 314 . 
Patients with Cushing's disease often develop thromboembolic complications. These patients have been shown to have a hypercoagulable and hypofibrinolytic state due to increased levels of several clotting factors, $\mathrm{vWF}, \alpha_{2}$-antiplasmine, and PAI-1315-317. Heart transplant recipients treated with corticosteroids had higher PAI-1 levels and less fibrinolytic capacity than recipients not administered corticosteroids ${ }^{318,319}$. Fibrinolytic capacity in renal transplant recipients treated with corticosteroids was comparable with patients with Cushing's disease ${ }^{313}$. After withdrawal of corticosteroids from a triple therapy regimen with CsA and AZA, fibrinolytic capacity improved with lower PAI-1 levels ${ }^{320,321}$. The effect of corticosteroids on PAI-1 may be mediated by their effect on glucose metabolism ${ }^{205}$, because PAI-1 levels are related to insulin resistance ${ }^{309,310}$. In vitro, corticosteroids were shown to inhibit the production of urokinase type plasminogen activator (u-PA) in a cell culture of aortic endothelial cells $s^{322}$. In a cell culture of umbilical vein endothelial cells, corticosteroids increased thrombinstimulated release of both $\mathrm{VWF}$ and PAI-1323. On the other hand, corticosteroids inhibited endotoxin-induced coagulation resulting in a hypocoagulable state. It was suggested that this was mediated by inhibition of monocyte-stimulated release of tissue factor ${ }^{324}$.

CsA is also associated with an impairment of fibrinolytic capacity. Fibrinolysis was impaired in CsA-treated patients compared with both AZA-treated patients ${ }^{325}$ and healthy controls ${ }^{326}$, by either a defective release of plasminogen activator from the vessel wall or high plasma levels of PAI-1325. After conversion from CsA to AZA, PAI-I activity decreased significantly ${ }^{327}$. Patients treated with CsA and corticosteroids had higher levels of $\mathrm{vWF}^{328}$ and fibrinogen ${ }^{329}$ than patients treated with AZA and corticosteroids. In addition, stepwise conversion from CsA to MMF lowered plasma $\mathrm{vWF}^{330}$. CsA-treated patients with aplastic anemia had higher levels of fibrinogen, vWF, and PAI-1 than aplastic anemia patients not treated with CsA ${ }^{331}$. CsA may also negatively influence hemostasis. Retrospectively, thromboembolic complications occurred more often in patients treated with CsA and corticosteroids than in patients treated with AZA and corticosteroids, and CsA-treated patients showed higher levels of factor VIII and fibrinogen ${ }^{332}$. However, these results could not be confirmed in a prospective design ${ }^{333}$. In vitro, hemostasis was increased in transplant recipients treated with CsA and corticosteroids, with or without AZA, compared with those receiving AZA and corticosteroids alone ${ }^{334}$. CsA enhanced the thrombin-stimulated release of both vWF and PAI-1 in a human umbilical vein endothelial cell culture s23,335 $^{32}$ and acted synergistically with corticosteroids in this experiment ${ }^{323}$.

Data on the other immunosuppressive drugs are very scarce. Fibrinogen levels were lower in TAC-treated patients than in CsA-treated patients in one study ${ }^{187}$. In another, conversion from $\mathrm{CsA}$ to TAC resulted in a significant decrease in plasma fibrinogen levels ${ }^{113}$. Conversion showed no effect on fibrinolysis 
parameters except for a slightly but significant decrease in urokinase plasminogen activatir (uPA) ${ }^{113}$.

\section{Conclusions}

An overview of the cardiovascular adverse effects of currently used immunosuppressive drugs is presented in Table 9.1.

Optimizing immunosuppression implies finding the optimal balance between efficacy and safety. Thus far, calcineurin inbitors appear to be the most potent immunosuppressive drugs for maintenance immunosuppression. However, they produce a number of undesirable adverse effects not only on renal function, but also on the cardiovascular system. TAC has several advantages over CsA. First, TAC is more potent than CsA in preventing acute rejections ${ }^{59,60,109,217}$. Although TAC-induced nephrotoxicity is histologically identical to that of CsA, more and more evidence is accumulating that, clinically, TAC may be less nephrotoxic. Moreover, TAC has a superior cardiovascular risk profile with regard to hypertension and hyperlipidemia. The enhanced risk of diabetes mellitus is clinically not apparent at commonly used maintenance dosages and can be managed by reducing the dose of TAC and early corticosteroid withdrawal.

Corticosteroids are undesirable for long-term immunosuppression. They induce endothelial dysfunction, hypertension, hyperlipidemia, diabetes, and result in a decreased fibrinolytic state. The benefit of an increase in HDL-cholesterol does not outweigh these adverse effects. Furthermore, they have several other undesirable adverse effects, such as weight gain and Cushing habitus, atrophy of the skin and vessel wall, and osteoporosis. The fear of an increase in acute or chronic rejection after withdrawal, which was demonstrated with $\mathrm{CsA}^{336}$, may be less with the more potent TAC.

Inhibitors of lymphocyte proliferation do not pose the risk of nephrotoxicity and have only minimal adverse cardiovascular effects. Prevention of anemia may be necessary in order to reduce LVH. However, the immunosuppressive power of these agents is less than that of calcineurin inhibitors. Therefore, they are almost always used in combination with the less desirable corticosteroids. MMF is more powerful than AZA, and because it does not interfere with DNA, the risk of developing malignancies may be less. Recently, the use of MMF was indeed associated with a reduction in the number of post-transplant lymphoproliferative disorders ${ }^{337}$. In selected series of low-risk transplant recipients, MMF as baseline maintenance immunosuppression has shown favourable results 338,339 ; however, its real value still needs to be determined.

RAPA is another promising agent because of its beneficial effects on intimal proliferation ${ }^{340-346}$. However, data on firm cardiovascular endpoints are lacking. In addition, it is still unclear whether the induced hyperlipidemia counteracts the 


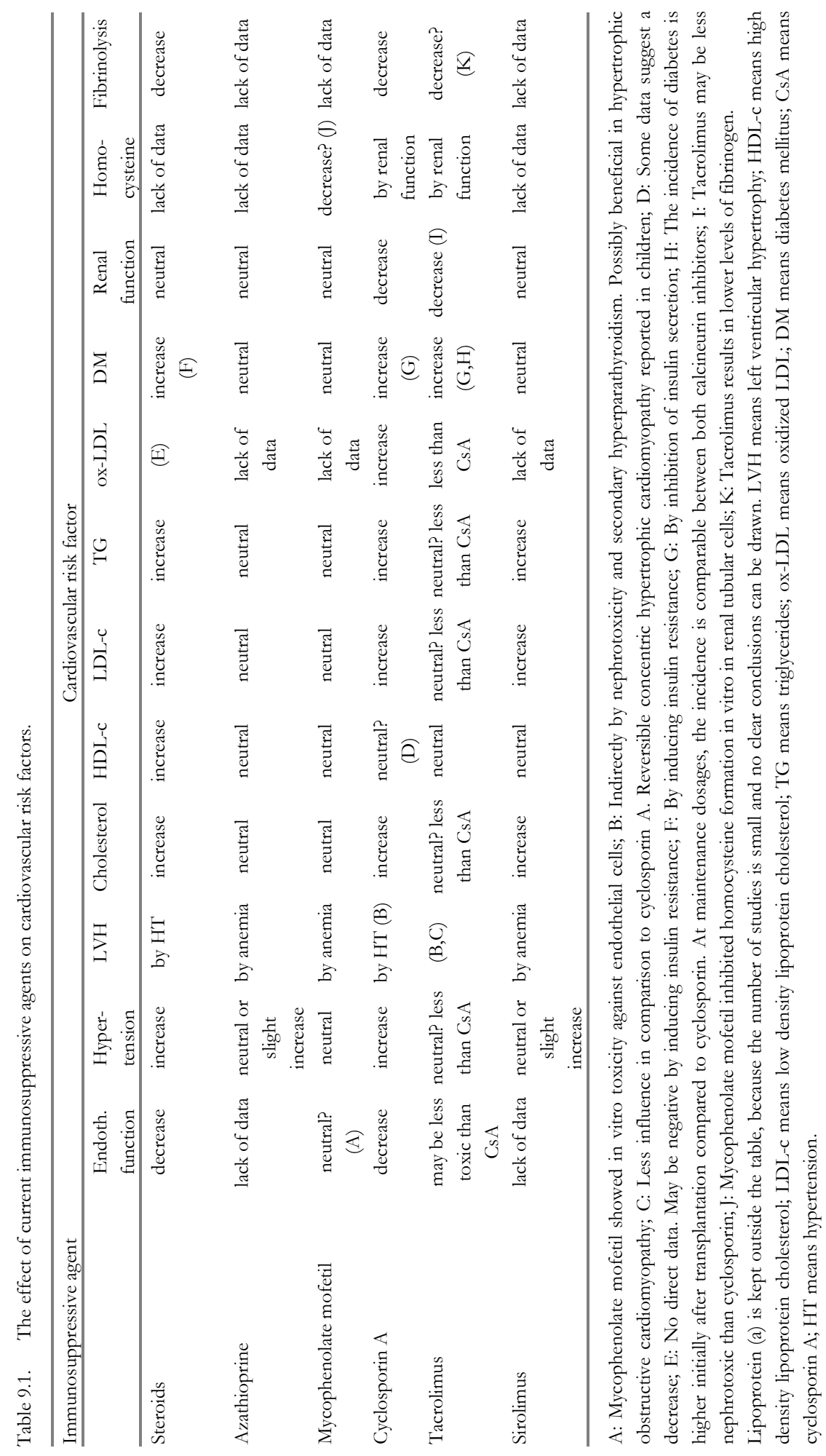


potential benefits. Care should be taken with regard to the development of druginduced pneumonia with RAPA 34,348 . Another advantage of the anti-proliferative characteristics of this compound may be a reduced risk of malignancies ${ }^{349-354}$.

The combination of RAPA and MMF seems an attractive alternative for avoiding calcineurin-induced nephrotoxicity. However, with this combination the incidence of acute rejection is higher ${ }^{198}$ than with tacrolimus-based immunosuppression $109,118,355$, initial attempts resulted in the development of severe mouth ulcers $^{356}$, and long-term data are lacking. Therefore, more data are necessary to establish the safety and efficacy of the combination of RAPA and MMF. 


\section{References}

1. Wolfe RA, Ashby VB, Milford EL, Ojo AO, Ettenger RE, Agodoa LYC, Held PJ and Port FK. Comparison of mortality in all patients on dialysis, patients on dialysis awaiting transplantation, and recipients of a first cadaveric transplant. N Engl J Med 1999;341:1725-30.

2. Schnuelle P, Lorenz D, Trede M and Woude FJ van der. Impact of renal cadaveric transplantation on survival in end-stage renal failure: evidence for reduced mortality risk compared with hemodialysis during long-term follow-up. J Am Soc Nephrol 1998;9:2135-41.

3. Ojo AO, Hanson JA, Meier-Kriesche H-U, Okechukwu CN, Wolfe RA, Leichtman AB, Agodoa LY, Kaplan B and Port FK. Survival in recipients of marginal cadaveric donor kidneys compared with other recipients and wait-listed transplant candidates. J Am Soc Nephrol 2001; 12:589-97.

4. Howard RJ, Patton PR, Reed AI, Hemming AW, Werf WJ van der, Pfaff WW, Srinivas TR and Scornik JC. The changing causes of graft loss and death after kidney transplantation. Transplantation 2002;73:1923-8.

5. Ojo AO, Hanson JA, Wolfe RA, Leichtman AB, Agodoa LY and Port FK. Long-term survival in renal transplant recipients with graft function. Kidney Int 2000;57:307-13.

6. Amemiya $\mathrm{H}$ and Itoh H. Mizoribine (Bredinin $\left.{ }^{\circledR}\right)$ : mode of action and effects on graft rejection. In Thomson AW and Starzl TE. Immunosuppressive Drugs: Developments in Antirejection Therapy. Edward Arnold. 1993:61-76.

7. Meier-Kriesche H-U, Arndorfer JA and Kaplan B. Association of antibody induction with short- and long-term cause specific mortality in renal transplantation. J Am Soc Nephrol 2002; 13:769-72.

8. $\quad$ Ross R. Atherosclerosis - An inflammatory disease. N Engl J Med 1999;340:115-26.

9. Frohlich J, Dobiasova M, Lear S and Lee KWJ. The role of risk factors in the development of atherosclerosis. Crit Rev Clin Lab Sci 2001;38:401-40.

10. Keaney JF jr. Atherosclerosis: from lesion formation to plaque activation and endothelial dysfunction. Mol Aspects Med 2000;21:99-166.

11. Gimbrone MA jr, Topper JN, Nagel T, Anderson KR and Garcia-Gardeña G. Endothelial dysfunction, hemodynamic forces, and atherogenesis. Ann N Y Acad Sci 2000;902:230-9.

12. Poredos P. Endothelial dysfunction in the pathogenesis of atherosclerosis. Int Angiol 2002;21: 109-16.

13. Glagov S, Zarins C, Giddens DP and Ku DN. Hemodynamics and atherosclerosis: Insights and perspectives gained from the studies of human arteries. Arch Pathol Lab Med 1988;112: 1018-33.

14. Schächinger V and Zeiher AM. Atherogenesis - recent insights into basic mechanisms and their clinical impact. Nephrol Dial Transplant 2002;17:2055-64.

15. Palmer RMJ, Ferrige AG and Moncada S. Nitric oxide accounts for the biological activity of endothelium-derived relaxing factor. Nature 1987;327:524-6.

16. Palmer RMJ, Ashton DS and Moncada S. Vascular endothelial cells synthesize nitric oxide from L-arginine. Nature 1988;329:664-6.

17. John $\mathrm{S}$ and Schmieder RE. Impaired endothelial function in arterial hypertension and hypercholesterolemia: potential mechanisms and differences. J Hypertens 2000;18:363-74. 
18. Cybulsky MI and Gimbrone MA jr. Endothelial expression of a mononuclear leukocyte adhesion molecule during atherogenesis. Science 1991;251:788-91.

19. Cushing SD, Berliner JA, Valente AJ, Territo MC, Navab M, Parhami F, Gerrity R, Schwartz CJ and Fogelman AM. Minimally modified low density lipoprotein induces monocyte chemotactic protein 1 in human endothelial cells and smooth muscle cells. Proc Natl Acad Sci U S A 1990;87:5134-8.

20. Jonasson L, Holm J, Skalli O, Bondjers G and Hansson GK. Regional accumulations of T cells, macrophages, and smooth muscle cells in the human atherosclerotic plaque. Arteriosclerosis 1986;6:131-8.

21. Wal AC van der, Das PK, Bentz van de Berg D, Loos CM van der and Becker AE. Atherosclerotic lesions in humans: in situ immunophenotypic analysis suggesting an immune mediated response. Lab Invest 1989;61:166-70.

22. Napoli C, D'Armiento FP, Mancini FP, Postiglioni A, Witztum JL, Palumbo G and Palinski W. Fatty streak formation occurs in human fetal aortas and is greatly enhanced by maternal hypercholesterolemia: intimal accumulation of low density lipoprotein and its oxidation precede monocyte recruitment into early atherosclerotic lesions. J Clin Invest 1997;100: 2680-90.

23. Stary HC, Chandler AB, Glagov S, Guyton JR, Insull W jr, Rosenfeld ME, Schaffer SA, Schwartz CJ, Wagner WD and Wissler RW. A definition of initial, fatty streak, and intermediate lesions of atherosclerosis: a report from the Comittee on Vascular Lesions of the Council on Arteriosclerosis, American Heart Association. Circulation 1994;89:2462-78.

24. Quinn MT, Parthasarathy S, Fong LG and Steinberg D. Oxidatively modified low density lipoproteins: a potential role in recruitment and retention of monocytes/macrophages during atherogenesis. Proc Natl Acad Sci U S A 1987;84:2995-8.

25. McMurray HF, Parthasarathy $\mathrm{S}$ and Steinberg D. Oxidatively modified low density lipoprotein is a chemoattractant for human T lymphocytes. J Clin Invest 1993;92:1004-8.

26. Parums DV, Brown DL and Mitchinson MJ. Serum antibodies to oxidized low-density lipoprotein and ceroid in chronic periaortitis. Arch Pathol Lab Med 1990; 14:383-7.

27. Salonen JT, Ylä-Herttuala S, Yamamoto R, Butler S, Korpela H, Salonen R, Nyyssonen K, Palinski W and Witztum JL. Autoantibody against oxidised LDL and progression of carotid atherosclerosis. Lancet 1992;339:883-7.

28. Klimov AN, Denisenko AD, Popov AV, Nagornev VA, Pleskov VM, Vinogradov AG, Denisenko TV, Magracheva EYa, Kheifes GM and Kuznetzov. Lipoprotein-antibody immune complexes. Their catabolism and role in foam cell formation. Atherosclerosis 1985;58:1-15.

29. Griffith RL, Virella GT, Stevenson HC and Lopes-Virella MF. Low density lipoprotein metabolism by human macrophages activated with low density lipoprotein immune complexes. A possible mechanism of foam cell formation. J Exp Med 1988;168:1041-59.

30. Glagov S, Weisenberg E, Zarins CK, Stankunavicius R and Kolettis GJ. Compensatory enlargement of human atherosclerotic coronary arteries. N Engl J Med 1987;316:1371-5.

31. Kiechl S and Willeit J for the Bruneck Study Group. The natural course of atherosclerosis. Part I: Incidence and progression. Arterioscler Thromb Vasc Biol 1999;19:1484-90.

32. London GM and Drüeke TB. Atherosclerosis and arteriosclerosis in chronic renal failure. Kidney Int 1997;51:678-95.

33. Hodis HN, Mack WJ, LaBree L, Selzer RH, Liu C-R, Liu C-H and Azen SP. The role of carotid arterial Intima-Media Thickness in predicting clinical coronary events. Ann Intern Med 1998;128:262-9. 
34. Blacher J, Guerin AP, Pannier B, Marchais SJ, Safar ME and London GM. Impact of aortic stiffness on survival in end-stage renal disease. Circulation 1999;99:2434-9.

35. Blacher J, Safar ME, Guerin AP, Pannier B, Marchais SJ and London GM. Aortic pulse wave velocity index and mortality in end-stage renal disease. Kidney Int 2003;63:1852-60.

36. Roman MJ, Saba PS, Pini R, Spitzer M, Pickering TG, Rosen S, Alderman MH and Devereux RB. Parallel cardiac and vascular adaptation in hypertension. Circulation 1992;86:1909-18.

37. London GM. The concept of ventricular/vascular coupling: functional and structural alterations of the heart and arterial vessels go in parallel. Nephrol Dial Transplant 1998;13: 250-3.

38. O'Rourke M. Mechanical principles in arterial disease. Hypertension 1995; 6:2-9.

39. Panza JA, Quyyumi AA, Brush JE and Epstein SE. Abnormal endothelium-dependent vascular relaxation in patients with essential hypertension. N Engl J Med 1990;323:22-7.

40. Higashi Y, Sasaki S, Nakagawa K, Matsuura H, Oshima T and Chayama K. Endothelial function and oxidative stress in renovascular hypertension. N Engl J Med 2002;346:1954-62.

41. Chowienczyk PJ, Watts GF, Cockcroft JR and Ritter JM. Impaired endothelium-dependent vasodilation of forearm resistance vessels in hypercholesterolaemia. Lancet 1992;340:1430-2.

42. Johnstone MT, Creager SJ, Scales KM, Cusco JA, Lee BK and Creager MA. Impaired endothelium-dependent vasodilation in patients with insulin-dependent diabetes mellitus. Circulation 1993;88:2510-6.

43. Celermajer DS, Sorensen KE, Georgakopoulos D, Bull C, Thomas O, Robinson J and Deanfield JE. Cigarette smoking is associated with dose-related and potentially reversible impairment of endothelium-dependent dilation in healthy young adults. Circulation 1993;88: 2149-55.

44. Pannier B, Guerin AP, Marchais SJ, Metivier F, Safar ME and London GM. Postischemic vasodilation, endothelial activation, and cardiovascular remodeling in end-stage renal disease. Kidney Int 2000;57:1091-99.

45. Bolton CH, Downs LG, Victory JGG, Dwight JF, Tomson CRV, Mackness MI and Pinkney $\mathrm{JH}$. Endothelial dysfunction in chronic renal failure: roles of lipoprotein oxidation and proinflammatory cytokines. Nephrol Dial Transplant 2001;16:1189-97.

46. Annuk M, Lind L, Linde T and Fellström B. Impaired endothelium-dependent vasodilatation in renal failure in humans. Nephrol Dial Transplant 2001;16:302-6.

47. Celermajer DS, Sorensen K, Ryalls M, Robinson J, Thomas O, Leonard JV and Danfield JE. Impaired endothelial function occurs in the systemic arteries of children with homozygous homocystinuria but not in their heterozygous parents. J Am Coll Cardiol 1993;22:854-8.

48. Stühlinger MC, Oka RK, Graf EE, Schmölzer I, Upson BM, Kapoor O, Szuba A, Malinow MR, Wascher TC, Pachinger O and Cooke JP. Endothelial dysfunction induced by hyperhomocyst(e)inemia. Role of asymmetric dimethylarginine. Circulation 2003; 08:933-8.

49. Graham A, Hogg N, Kalyanaraman B, O'Leary V, Darley-Usmar V and Moncada S. Peroxynitrite modification of low-density lipoprotein leads to recognition by the macrophage scavenger receptor. FEBS Letters 1993;330:181-5.

50. Chambers JC, Ueland PM, Wright M, Doré CJ, Refsum H and Kooner JS. Investigation of relationship between reduced, oxidized, and protein-bound homocysteine and vascular endothelial function in healthy human subjects. Circ Res 2001;89:187-92.

51. Dawber TR and Kannel WB. The Framingham study. An epidemiological approach to coronary heart disease. Circulation 1966;34:553-5. 
52. Kasiske BL. Risk factors for accelerated atherosclerosis in renal transplant recipients. Am J Med 1988;84:985-92.

53. Kasiske BL, Chakkera HA and Roel J. Explained and unexplained ischemic heart disease risk after renal transplantation. J Am Soc Nephrol 2000;11:1735-43.

54. Manske CL, Wang Y, Rector T, Wilson RF and White CW. Coronary revascularisation in insulin-dependent diabetic patients with chronic renal failure. Lancet 1992;340:998-1002.

55. Kasiske BL, Guijarro C and Massy ZA. Cardiovascular disease after renal transplantation. J Am Soc Nephrol 1996;7:158-65.

56. Foley RN, Parfrey PS and Sarnak MJ. Clinical epidemiology of cardiovascular disease in chronic renal disease. Am J Kidney Dis 1998;32(S3):S112-9.

57. Hollander AA, Saase JL van, Kootte AM, Dorp WT van, Bockel HJ van, Es LA van and Woude FJ van der. Beneficial effects of conversion from cyclosporin to azathioprine after kidney transplantation. Lancet 1995;345:610-4.

58. Bakker RC, Hollander AAMJ, Mallat MJK, Bruijn JA, Paul LC and Fijter JW de. Conversion from cyclosporine to azathioprine at three months reduces the incidence of chronic allograft nephropathy. Kidney Int 2003;64:1027-34.

59. Pirsch JD, Miller J, Deierhoi MH, Vincenti F and Filo RS for the FK506 Kidney Transplant Study Group. A comparison of tacrolimus (FK506) and cyclosporine for immunosuppression after cadaveric renal transplantation. Transplantation 1997;63:977-83.

60. Mayer AD, Dmitrewski J, Squifflet J-P, Besse T, Grabensee B, Klein B, Eigler FW, Heemann U, Pichlmayr R, Behrend M, Vanrenterghem Y, Donck J, Hooff J van, Christiaans M, Morales JM, Andres A, Johnson RWG, Short C, Buchholz B, Rehmert N, Land W, Schleibner S, Forsythe JLR, Talbot D, Neumayer H-H, Hauser I, Ericzon B-G, Brattström C, Claesson K, Mühlbacher F and Pohanka E. Multicenter randomized trial comparing tacrolimus (FK 506) and cyclosporin in the prevention of renal allograft rejection. A report of the European Tacrolimus Multicenter Renal Study Group. Transplantation 1997;64:436-43.

61. Schnitzler MA, Craig KE, Hardinger KE, Lowell JA and Brennan DC. Mycophenolate mofetil is associated with less death with function than azathioprine in cadaveric renal transplantation. Nephrol Dial Transplant 2003;18:1197-1200.

62. Passauer J, Büssemaker E, Lassig G and Gross P. Kidney transplantation improves endothelium-dependent vasodilation in patients with endstage renal disease. Transplantation 2003;75:1907-10.

63. Oflaz H, Pusuroglu H, Genchallac H, Demirel S, Bugra Z, Sever MS and Yildiz A. Endothelial function is more impaired in hemodialysis patients than renal transplant recipients. Clin Transplant 2003;17:528-33.

64. Iuchi T, Akaike M, Mitsui T, Ohshima Y, Shintani Y, Azuma H and Matsumoto T. Glucocorticoid excess induces superoxide production in vascular endothelial cells and elicits vascular endothelial dysfunction. Circ Res 2003;92:81-7.

65. Morris STW, McMurray JJV, Rodger RSC, Farmer R and Jardine AG. Endothelial dysfunction in renal transplant recipients maintained on cyclosporin. Kidney Int 2000;57:1100-6.

66. Dorpel MA van den, Meiracker AH van den, Lameris TW, Weimar W and Man in 't Veld AJ. Forearm vasorelaxation in hypertensive renal transplant patients. The impact of withdrawal of cyclosporine. J Hypertens 1998;16:331-7.

67. Galle J, Lehman-Bodem C, Hübner U, Heinloth A and Wanner C. CyA and OxLDL cause endothelial dysfunction in isolated arteries through endothelin-mediated stimulation of $\mathrm{O}_{2}$ formation. Nephrol Dial Transplant 2000;15:339-46. 
68. Ovuworie CA, Fox ER, Chow C-M, Pascual M, Shih VE, Picard MH and Tolkoff-Rubin NE. Vascular endothelial function in cyclosporine and tacrolimus treated renal transplant recipients. Transplantation 2001;72:1385-8.

69. Oflaz H, Turkmen A, Kazancioglu R, Kayacan SM, Bunyak B, Genchallac H, Erol B, Mercanoglu F, Umman S and Sever MS. The effect of calcineurin inhibitors on endothelial function in renal transplant recipients. Clin Transplant 2003;17:212-6.

70. Wilasrusmee C, Da Silva M, Singh B, Siddiqui J, Bruch D, Kittur S, Wilasrusmee S and Kittur DS. Morphological and biochemical effects of immunosuppressive drugs in a capillary tube assay for endothelial dysfunction. Clin Transplant 2003;17:6-12.

71. Wilasrusmee C, Da Silva M, Siddiqui J, Bruch D, Kittur S, Wilasrusmee S and Kittur DS. Role of Endothelin-1 in microvascular dysfunction caused by cyclosporin A. J Am Coll Surg 2003; 196:584-91.

72. Wilson PWF, D'Agostino RB, Levy D, Belanger AM, Silbershatz H and Kannel WB. Prediction of coronary heart disease using risk factor categories. Circulation 1998;97:1837-47.

73. Mange KC, Cizman B, Joffe M and Feldman HI. Arterial hypertension and renal allograft survival. J Am Med Assoc 2000;283:633-8.

74. Opelz G, Wujciak T and Ritz E for the Collaborative Transplant Study. Association of chronic kidney graft failure with recipient blood pressure. Kidney Int 1998;53:217-22.

75. Neal B, MacMahon S and Chapman N for the Blood Pressure Lowering Treatment Trialists' Collaboration. Effects of ACE inhibitors, calcium antagonists, and other blood-pressurelowering drugs: results of prospectively designed overviews of randomised trials. Lancet 2000; 356:1955-64.

76. Hoes AW, Grobbee DE and Lubsen J. Does drug treatment improve survival? Reconciling the trials in mild-to-moderate hypertension. J Hypertens 1995;13:805-11.

77. Kasiske BL, Vazquez MA, Harmon WE, Brown RS, Danovitch GM, Gaston RS, Roth D, Scandling JD jr. and Singer GG for the American Society of Nephrology. Recommendations for the outpatient surveillance of renal transplant recipients. J Am Soc Nephrol 2000;11:S1-6.

78. Pérez Fontán M, Rodriguez-Carmona A, Garcia Falcón T, Fernández Rivera C and Valdés F. Early immunologic and nonimmunologic predictors of arterial hypertension after renal transplantation. Am J Kidney Dis 1999;33:21-8.

79. Peschke B, Scheuermann, Geiger H, Bölscher S, Kachel H-G and Lenz T. Hypertension is associated with hyperlipidemia, coronary heart disease and chronic graft failure in kidney transplant recipients. Clin Nephrol 1999;51:290-5.

80. Kasiske BL. Ischemic heart disease after renal transplantation. Kidney Int 2002;61:356-69.

81. Midtvedt $\mathrm{K}$ and Hartmann A. Hypertension after kidney transplantation: are treatment guidelines emerging? Nephrol Dial Transplant 2002; 7:1166-9.

82. Warholm C, Wilczek $\mathrm{H}$ and Petterson E. Hypertension two years after renal transplantation: causes and consequences. Transpl Int 1995;8:286-92.

83. Huysmans FThM, Hoitsma AJ and Koene RAP. Factors determining the prevalence of hypertension after renal transplantation. Nephrol Dial Transplant 1987;2:34-8.

84. Whitworth JA, Mangos GJ and Kelly JJ. Cushing, cortisol, and cardiovascular disease. Hypertension 2000;36:912-6.

85. Borne P van de, Gelin M, Stadt J van de and Degaute J-P. Circadian rhythms of blood pressure after liver transplantation. Hypertension 1993;21:398-405. 
86. Fallo F, Paoletta A, Tona F, Boscaro M and Sonino N. Response of hypertension to conventional antihypertensive treatment and/or steroidogenesis inhibitors in Cushing's syndrome. J Intern Med 1993;234:595-8.

87. Brinker KR, Dickerman RM, Gonwa TA, Hull AR, Langley JW, Long DL, Nesser DA, Trevino G, Velez RL and Vergne-Marini PJ. A randomized trial comparing double-drug and triple drug therapy in primary cadaveric renal transplants. Transplantation 1990;50:43-9.

88. Sollinger HW for the U.S. Renal Transplant Mycophenolate Mofetil Study Group. Mycophenolate mofetil for the prevention of acute rejection in primary cadaveric renal allograft recipients. Transplantation 1995;60:225-32.

89. Schnuelle P, Heide JH van der, Tegzess A, Verburgh CA, Paul LC, Woude FJ van der and Fijter JW de. Open randomized trial comparing early withdrawal of either cyclosporine or mycophenolate mofetil in stable renal transplant recipients initially treated with a triple drug regimen. J Am Soc Nephrol 2002;13:536-43.

90. Chapman JR, Marcen R, Arias M, Raine AEG, Dunnill MS and Morris PJ. Hypertension after renal transplantation. Transplantation 1987;43:860-4.

91. Sutherland DE, Fryd DS, Strand MH, Canafax DM, Ascher NL, Payne WD, Simmons RL and Najarian JS. Results of the Minnesota randomized prospective trial of cyclosporine versus azathioprine-antilymphocyte globulin for immunosuppression in renal allograft recipients. Am J Kidney Dis 1985;5:318-27.

92. Jarowenko MV, Flechner SM, Buren CT van, Lorber MI and Kahan BD. Influence of cyclosporine on posttransplant blood pressure response. Am J Kidney Dis 1987;10:98-103.

93. Hueso M, Bover J, Serón D, Gil-Vernet S, Sabaté I, Fulladosa X, Ramos R, Coli O, Alsina J and Grinyó JM. Low-dose cyclopsorine and mycophenolate mofetil in renal allograft recipients with suboptimal renal function. Transplantation 1998;66:1727-31.

94. Versluis DJ, Wenting GJ, Derkx FHM, Schalekamp MADH, Jeekel J and Weimar W. Who should be converted from cyclosporine to conventional immunosuppression in kidney transplantation, and why. Transplantation 1987;44:387-9.

95. Dorpel MA van den, Meiracker AH van den, Lameris TW, Boomsma F, Levi M, Man in 't Veld AJ and Schalekamp MADH. Cyclosporine A impairs the nocturnal blood pressure fall in renal transplant recipients. Hypertension 1996;28:304-7.

96. Hilbrands LB, Hoitsma AJ and Koene RAP. Randomized, prospective trial of cyclosporin monotherapy versus azathioprine-prednisone from three months after renal transplantation. Transplantation 1996;61:1038-46.

97. Schrama YC, Joles JA, Tol A van, Boer P, Koomans HA and Hené RJ. Conversion to mycophenolate mofetil in conjunction with stepwise withdrawal of cyclosporine in stable renal transplant recipients. Transplantation 2000;69:376-83.

98. Johnson RW, Kreis H, Oberbauer R, Brattström C, Claesson K and Eris J. Sirolimus allows early cyclosporine withdrawal in renal transplantation resulting in improved renal function and lower blood pressure. Transplantation 2001;72:777-86.

99. Oberbauer R, Kreis H, Johnson RWG, Mota A, Claesson K, Ruiz JC, Wilczek H, Jamieson N, Henriques AC, Paczek L, Chapman J and Burke JT for the Rapamune Maintenance Regimen Study Group. Long-term improvement in renal function with sirolimus after early cyclosporine withdrawal in renal transplant recipients: 2-year results of the Rapamune Maintenance Regimen Study. Transplantation 2003;76:364-70. 
100. Ruggenenti P, Perico N, Mosconi L, Gaspari F, Begigni A, Amuchastegui CS, Bruzzi I and Remuzzi G. Calcium channel blockers protect transplant patients from cyclosporine-induced daily renal hypoperfusion. Kidney Int 1993;43:706-11.

101. Kivlighn SD, Gabel RA and Siegl PKS. Effects of BQ-123 on renal function and acute cyclosporine-induced renal dysfunction. Kidney Int 1994;45:131-6.

102. Kirk AD, Jacobson LM, Heisey DM, Fass NA, Sollinger HW and Pirsch JD. Posttransplant diastolic hypertension. Associations with intragraft transforming growth factor- $\beta$, endothelin and renin transcription. Transplantation 1997;64:1716-20.

103. Sturrock NDC and Struthers AD. Hormonal and other mechanisms involved in the pathogenesis of cyclosporin-induced nephrotoxicity and hypertension in man. Clin Sci 1994; 86:1-9.

104. Galiatsou E, Morris ST, Jardine AG, Rodger RS, Watson MA and Elliott HL. Cardiac and vascular abnormalities in renal transplant patients: differential effects of cyclosporin and azathioprine. J Nephrol 2000;13:185-92.

105. Textor SC, Wiesner R, Wilson DJ, Porayko M, Romero JC, Burnett JC jr, Gores G, Hay E, Dickson ER and Krom RA. Systemic and renal hemodynamic differences between FK506 and cyclopsorine in liver transplant recipients. Transplantation 1993;55:1332-9.

106. Taler SJ, Textor SC, Canzanello VJ, Schwartz L, Porayko M, Wiesner RH and Krom RA. Role of steroid dose in hypertension early after liver transplantation with tacrolimus (FK506) and cyclosporine. Transplantation 1996;62:1588-92.

107. Radermacher J, Meiners M, Bramlage C, Kliem V, Behrend M, Schlitt HJ, Pichlmayr R, Koch $\mathrm{KM}$ and Brunkhorst R. Pronounced renal vasoconstriction and systemic hypertension in renal transplant recipients treated with cyclosporin A versus FK 506. Transpl Int 1998;11:3-10.

108. Hohage H, Brückner D, Arlt M, Buchholz B, Zidek W and Spieker C. Influence of cyclosporin $\mathrm{A}$ and FK506 on $24 \mathrm{~h}$ blood pressure monitoring in kidney transplant recipients. Clin Nephrol 1996;45:342-4.

109. Margreiter $\mathrm{R}$ for the European Tacrolimus vs Ciclosporin Microemulsion Renal Transplantation Study Group. Efficacy and safety of tacrolimus compared with ciclosporin microemulsion in renal transplantation: a randomised multicentre study. Lancet 2002;359: 741-6.

110. Kohnle M, Zimmermann U, Lütkes P, Albrecht K-H, Philipp T and Heemann U. Conversion from cyclosporin A to tacrolimus after kidney transplantation due to hyperlipidemia. Transpl Int 2000;13 (S1):S345-8.

111. Ligtenberg G, Hené RJ, Blankestijn PJ and Koomans HA. Cardiovascular risk factors in renal transplant patients: Cyclosporin A versus tacrolimus. J Am Soc Nephrol 2001;12:368-73.

112. Klein IH, Abrahams A, Ede T van, Hené RJ, Koomans HA and Ligtenberg G. Different effects of tacrolimus and cyclosporine on renal hemodynamics and blood pressure in healthy subjects. Transplantation 2002;73:732-6.

113. Artz MA, Boots JMM, Ligtenberg G, Roodnat JI, Christiaans MHL, Vos PF, Blom HJ, Sweep FCGJ, Demacker PNM and Hilbrands LB. Improved cardiovascular risk profile and renal function in renal transplant patients after randomized conversion from cyclosporine to tacrolimus. J Am Soc Nephrol 2003;14:1880-8.

114. Artz MA, Boots JMM, Ligtenberg G, Roodnat JI, Christiaans MHL, Vos PF, Moons P, Borm $\mathrm{G}$ and Hilbrands LB. Conversion from cyclosporine to tacrolimus improves quality-of-life indices, renal graft function, and cardiovascular risk profile. Am J Transplant 2004;4:937-45. 
115. Martinez Castelao A, Ramos R, Seron D, Gil-Vernet S, Fiol C, Gomez-Gerique N, Yzaguirre MT, Hurtado I, Sabate I, Alsina J and Grinyo JM. Effect of cyclosporin and tacrolimus on lipoprotein oxidation after renal transplantation. Nefrologia 2002;22:364-9.

116. Kahan BD, Julian BA, Pescovitz MD, Vanrenterghem Y and Neylan J for the Rapamune Study Group. Sirolimus reduces the incidence of acute rejection episodes despite lower cyclosporine doses in Caucasian recipients of mismatched primary renal allografts: A phase II trial. Transplantation 1999;68:1526-32.

117. Groth CG, Bäckman L, Morales J-M, Calne R, Kreis H, Lang P, Touraine J-L, Claesson K, Campistol JM, Durand D, Wramner L, Brattström C and Charpentier B for the Sirolimus European Renal Transplant Study Group. Sirolimus (Rapamycin)-based therapy in human renal transplantation. Transplantation 1999;67:1036-42.

118. Gonwa T, Mendez R, Yang HC, Weinstein S, Jensik S and Steinberg S for the Prograf Study Group. Randomized trial of tacrolimus in combination with sirolimus or mycophenolate mofetil in kidney transplantation: Results at 6 months. Transplantation 2003;75:1213-20.

119. Levin A, Singer J, Thompson CR, Ross H and Lewis M. Prevalent left ventricular hypertrophy in the predialysis population: Identifying opportunities for intervention. Am J Kidney Dis 1996;27:347-54.

120. Foley RN, Parfrey PS, Harnett JD, Kent GM, Martin CJ, Murray DC and Barre PE. Clinical and echocardiographic disease in patients starting end-stage renal disease therapy. Kidney Int 1995;47:186-92.

121. Levy D, Garrison RJ, Savage DD, Kannel WB and Castelli WP. Prognostic implications of echocardiographically dtermined left ventricular mass in the Framingham heart study. N Engl J Med 1990;322:1561-6.

122. Middleton RJ, Parfrey PS and Foley RN. Left ventricular hypertrophy in the renal patient. J Am Soc Nephrol 2001;12:1079-84.

123. McGregor E, Jardine AG, Murray LS, Dargie HJ, Rodger RSC, Junor BJR, McMillan MA and Briggs JD. Pre-operative echocardiographic abnormalities and adverse outcome following renal transplantation. Nephrol Dial Transplant 1998;13:1499-505.

124. Rigatto C, Foley RN, Kent GM, Guttmann R and Parfrey PS. Long-term changes in left ventricular hypertrophy after renal transplantation. Transplantation 2000;70:570-5.

125. Peteiro J, Alvarez N, Calviño R, Penas M, Ribera F and Castro Beiras A. Changes in left ventricular mass and filling after renal transplantation are related to changes in blood pressure: An echocardiographic and pulsed doppler study. Cardiology 1994;85:273-83.

126. Ritter O, Hack S, Schuh K, Röthlein N, Perrot A, Osterziel KJ, Schulte HD and Neyses L. Calcineurin in human heart hypertrophy. Circulation 2002;105:2265-9.

127. Molkentin JD, Lu J-R, Antos CL, Markham B, Richardson J, Robbins J, Grant SR and Olson EN. A calcineurin-dependent transcriptional pathway for cardiac hypertrophy. Cell 1998;93:215-28.

128. Sussman MA, Lim HW, Gude N, Taigen T, Olson EN, Robbins J, Colbert MC, Gualberto A, Wieczorek DF and Molkentin JD. Prevention of cardiac hypertrophy in mice by calcineurin inhibition. Science 1998;281:1690-3.

129. Shimoyama M, Hayashi D, Takimoto E, Zou Y, Oka T, Uozumi H, Kudoh S, Shibasaki F, Yazaki Y, Nagai R and Komuro I. Calcineurin plays a critical role in pressure overload-induced cardiac hypertrophy. Circulation 1999;100:2449-54. 
130. Shimoyama M, Hayashi D, Zou Y, Takimoto E, Mizukami M, Monzen K, Kudoh S, Hiroi Y, Yazaki Y, Nagai R and Komuro I. Calcineurin inhibitor attenuates the development and induces the regression of cardiac hypertrophy in rats with salt-sensitive hypertension. Circulation 2000;102:1996-2004.

131. Atkinson P, Joubert G, Barron A, Grant D, Paradis K, Seidman E, Wall W, Rosenberg H, Howard J, Williams S and Stiller C. Hypertrophic cardiomyopathy associated with tacrolimus in paediatric transplant patients. Lancet 1995;345:894-6.

132. Nakata Y, Yoshibayashi M, Yonemura T, Uemoto S, Inomata Y, Tanaka K and Furusho K. Tacrolimus and myocardial hypertrophy. Transplantation 2000;69:1960-2.

133. Espino G, Denney J, Furlong T, Fitzsimmons W and Nash RA. Graft-versus-host disease. Assessment of myocardial hypertrophy by echocardiography in adult patients receiving tacrolimus or cyclosporine therapy for prevention of acute GVHD. Bone Marrow Transplant 2001;28:1097-103.

134. Schwitter J, De Marco T, Globits S, Sakuma H, Klinski C, Chatterjee K, Parmley WW and Higgins CB. Influence of felodipine on left ventricular hypertrophy and systolic function in orthotopic heart transplant recipients: possible interaction with cyclosporin medication. J Heart Lung Transplant 1999;18:1003-13.

135. Besouw NM van, Mast BJ van der, Smak Gregoor PJH, Hesse CJ, IJzermans JNM, Gelder T van and Weimar W. Effect of mycophenolate mofetil on erythropoiesis in stable renal transplant patients is correlated with mycophenolic acid trough levels. Nephrol Dial Transplant 1999;14:2710-3.

136. Pascual $\mathrm{J}$ and Ortuño $\mathrm{J}$ for the Spanish and Italian Tacrolimus Study Group. Simple Tacrolimus-based immunosuppressive regimens following renal transplantation: A large multicenter comparison between double and triple therapy. Transplant Proc 2002;34:89-91.

137. Kahan BD for the Rapamune US Study Group. Efficacy of sirolimus compared with azathioprine for reduction of acute renal allograft rejection: a randomised multicentre study. Lancet 2000;356:194-202.

138. Mihatsch MJ, Kyo M, Morozumi K, Yamaguchi Y, Nickeleit V and Ryffel B. The side effects of ciclosporin-A and tacrolimus. Clin Nephrol 1998;49:356-63.

139. LaRosa JC, He J and Vupputuri S. Effect of statins on risk of coronary disease. A meta-analysis of randomized controlled trials. J Am Med Assoc 1999;282:2340-6.

140. Pedersen TR, Olsson AG, Færgeman O, Kjekshus J, Wedel H, Berg K, Wilhelmsen L, Haghfelt T, Thorgeirsson G, Pyörälä K, Miettinen T, Christophersen B, Tobert JA, Musliner TA and Cook TJ for the Scandinavian Simvastatin Survival Study Group. Lipoprotein changes and reduction in the incidence of major coronary heart disease events in the Scandinavian Simvastatin Survival Study (4S). Circulation 1998;97:1453-60.

141. Aakhus S, Dahl K and Widerøe TE. Cardiovascular morbidity and risk factors in renal transplant patients. Nephrol Dial Transplant 1999;14:648-54.

142. Roodnat JI, Mulder PGH, Zietse R, Rischen-Vos J, Riemsdijk IC van, IJzermans JNM and Weimar W. Cholesterol as an independent predictor of outcome after renal transplantation. Transplantation 2000;69:1704-10.

143. Booth JC, Joseph JT and Jindal RM. Influence of hypercholesterolemia on patient and graft survival in recipients of kidney transplants. Clin Transplant 2003;17:101-7.

144. Cosio FG, Pesavento TE, Pelletier RP, Henry M, Ferguson RM, Kim S and Lemeshow S. Patient survival after renal transplantation III: The effect of statins. Am J Kidney Dis 2002;40: 638-43. 
145. Dimény E, Wahlberg J, Lithell H and Fellström B. Hyperlipidaemia in renal transplantation risk factor for long-term graft outcome. Eur J Clin Invest 1995;25:574-83.

146. Wissing KM, Abramowicz D, Broeders $\mathrm{N}$ and Vereerstraeten P. Hypercholesterolemia is associated with increased kidney graft loss caused by chronic rejection in male patients with previous acute rejection. Transplantation 2000;70:464-72.

147. Holdaas H, Fellström B, Jardine AG, Holme I, Nyberg G, Fauchald P, Grönhagen-Riska C, Madsen S, Neumayer H-H, Cole E, Maes B, Ambühl P, Olsson AG, Hartmann A, Solbu DO and Pedersen TR on behalf of the Assessment of LEscol in Renal Transplantation (ALERT) Study Investigators. Effect of fluvastatin on cardiac outcomes in renal transplant recipients: a multicentre, randomised, placebo-controlled trial. Lancet 2003;361:2024-31.

148. Sever PS, Dahlöf B, Poulter NR, Wedel H, Beevers G, Caulfield M, Collins R, Kjeldsen SE, Kristinsson A, McInnes GT, Mehlsen J, Nieminen M, O'Brien E and Ostergren J for the ASCOT Investigators. Prevention of coronary and stroke events with atorvastatin in hypertensive patients who have average or lower-than-average cholesterol concentrations, in the Anglo-Scandinavian Cardiac Outcomes Trial--Lipid Lowering Arm (ASCOT-LLA): a multicentre randomised controlled trial. Lancet 2003;361:1149-58.

149. Aguilar-Salinas CA, Díaz-Polanco A, Quintana E, Macias N, Arellano A, Ramírez E, Ordóñez ML, Velásquez-Alva C, Gómez Pérez FJ, Alberú J and Correa-Rotter R. Genetic factors play an important role in the pathogenesis of hyperlipidemia post-transplantation. Am J Kidney Dis 2002;40:169-77.

150. Hricik DE and Schulak JA. Metabolic effects of steroid withdrawal in adult renal transplant recipients. Kidney Int 1993;43:S26-9.

151. Sévaux RGL de, Hilbrands LB, Tiggeler RGWL, Koene RAP and Hoitsma AJ. A randomised, prospective study on the conversion from cyclosporine-prednisone to cyclosporineazathioprine at 6 months after renal transplantation. Transpl Int 1998;11(S1):S322-4.

152. Squifflet J-P, Vanrenterghem Y, Hooff JP van, Salmela K, Rigotti P and the European Tacrolimus/MMF Transplantation Study Group. Safe withdrawal of corticosteroids or Mycophenolate Mofetil: Results of a large, prospective, multicenter, randomized study. Transplant Proc 2002;34:1584-6.

153. Hilbrands LB, Demacker PNM, Hoitsma AJ, Stalenhoef AFH and Koene RAP. The effects of cyclosporine and prednisone on serum lipid and (apo)lipoprotein levels in renal transplant recipients. J Am Soc Nephrol 1995;5:2073-81.

154. Chan MK, Varghese Z, Persaud JW, Fernando ON and Moorhead JF. The role of multiple pharmaco-therapy in the pathogenesis of hyperlipidemia after renal transplantation. Clin Nephrol 1981;15:309-13.

155. Ibels LS, Alfrey AC, Subryan V and Weil R 3rd. Hyperlipidemia following renal transplantation. Trans Am Soc Artif Intern Organs 1976;22:46-53.

156. Ibels LS, Simons LA, King JO, Williams PF, Neale FC and Stewart JH. Studies on the nature and causes of hyperlipidaemia in uraemia, maintenance dialysis and renal transplantation. Q J Med 1975;44:601-14.

157. Ponticelli C, Barbi GL, Cantaluppi A, Vecchi A de, Annoni G, Donati C and Cecchettin M. Lipid disorders in renal transplant recipients. Nephron 1978;20:189-95.

158. Diamant S and Shafrir E. Modulation of the activity of insulin-dependent enzymes of lipogenesis by glucocorticoids. Eur J Biochem 1975;53:541-6. 
159. Carpentier A, Patterson BW, Leung N and Lewis GF. Sensitivity to acute insulin-mediated suppression of plasma free fatty acids is not a determinant of fasting VLDL triglyceride secretion in healthy humans. Diabetes 2002;51:1867-75.

160. Chan MK, Persaud JW, Varghese Z, Fernando ON and Moorhead JF. Fat clearances and hyperlipidaemia in renal allograft recipients--the role of insulin resistance. Clin Chim Acta 1981;114:61-7.

161. Quiñones-Galvan A, Sironi AM, Baldi S, Galetta F, Garbin U, Fratta-Pasini A, Cominacini L and Ferrannini E. Evidence that acute insulin administration enhances LDL cholesterol susceptibility to oxidation in healthy humans. Arterioscler Thromb Vasc Biol 1999;19:2928-32.

162. Chan MK, Varghese Z and Moorhead JF. Lipid abnormalities in uremia, dialysis, and transplantation. Kidney Int 1981;19:625-37.

163. Henze K, Chait A, Albers JJ and Bierman EL. Hydrocortisone decreases the internalization of low density lipoprotein in cultured human fibroblasts and arterial smooth muscle cells. Eur J Clin Invest 1983;13:171-7.

164. Rayyes OA, Wallmark A and Florén C-H. Additive inhibitory effect of hydrocortisone and cyclosporine on low-density lipoprotein receptor activity in cultured HepG2 cells. Hepatology 1997;26:967-71.

165. Kancha RK and Hussain MM. Up-regulation of the low density lipoprotein receptor-related protein by dexamethasone in HepG2 cells. Biochim Biophys Acta 1996;1301:213-20.

166. Moulin P, Appel GB, Ginsberg HN and Tall AR. Increased concentration of plasma cholesteryl ester transfer protein in nephrotic syndrome: role in dyslipidemia. J Lipid Res 1992;33:1817-22.

167. Agellon LB, Walsh A, Hayek T, Moulin P, Jiang XC, Shelanski SA, Breslow JL and Tall AR. Reduced high density lipoprotein cholesterol in human cholesteryl ester transfer protein transgenic mice. J Biol Chem 1991;266:10796-801.

168. Hayek T, Chajek-Shaul T, Walsh A, Agellon LB, Moulin P, Tall AR and Breslow JL. An interaction between the human cholesteryl ester transfer protein (CETP) and apolipoprotein A-1 genes in transgenic mice results in a profound CETP-mediated depression of high density lipoprotein cholesterol levels. J Clin Invest 1992;90:505-10.

169. Kuster GM, Drexel H, Bleisch JA, Rentsch K, Pei P, Binswanger U and Amann FW. Relation of cyclopsorin blood levels to adverse effects on lipoproteins. Transplantation 1994;57: 1479-83.

170. Ballantyne CM, Podet EJ, Patsch WP, Harati Y, Appel V, Gotto AM and Young JB. Effects of cyclosporine therapy on plasma lipoprotein levels. J Am Med Assoc 1989;262:53-6.

171. Raine AE, Carter R, Mann JI and Morris PJ. Adverse effects of cyclosporin on plasma cholesterol in renal transplant recipients. Nephrol Dial Transplant 1988;3:458-63.

172. Dorpel MA van den, Ghanem H, Rischen-Vos J, Jansen H and Weimar W. Conversion from cyclosporin A to azathioprine treatment improves LDL oxidation in kidney transplant recipients. Kidney Int 1997;51:1608-12.

173. Harris KP, Russell GI, Parvin SD, Veitch PS and Walls J. Alterations in lipid and carbohydrate metabolism attributable to cyclosporin A in renal transplant recipients. Br Med J 1986;292: 16-9.

174. Abramowicz D, Manas D, Lao M, Vanrenterghem Y, Del Castillo D, Wijngaard P and Fung S for the Cyclosporine Withdrawal Study Group. Cyclosporine withdrawal from a mycophenolate mofetil-containing immunosuppressive regimen in stable kidney transplant recipients: a randomized, controlled study. Transplantation 2002;74:1725-34. 
175. Espino A, Lopez-Miranda J, Blanco-Cerrada J, Zambrana JL, Aumente MA, Paniagua JA, Blanco-Molina A, Jimenez-Pereperez JA, Rodriguez M and Pérez-Jiménez F. The effect of cyclopsorine and methylprednisolone on plasma lipoprotein levels in rats. J Lab Clin Med 1995;125:222-7.

176. Vathsala A, Weinberg RB, Schoenberg L, Grevel J, Goldstein RA, Buren CT van, Lewis RM and Kahan BD. Lipid abnormalities in cyclosporine-prednisone-treated renal transplant recipients. Transplantation 1989;48:37-43.

177. Quaschning T, Mainka T, Nauck M, Rump LC, Wanner C and Krämer-Guth A. Immunosuppression enhances atherogenicity of lipid profile after transplantation. Kidney Int 1999;56(S71):S235-7.

178. Vaziri ND, Liang K and Azad H. Effect of cyclopsorine on HMG-CoA reductase, cholesterol 7alpha-hydroxylase, LDL receptor, HDL receptor, VLDL receptor, and lipoprotein lipase expressions. J Pharmacol Exp Ther 2000;294:778-83.

179. López-Miranda J, Vilella E, Pérez-Jiménez F, Espino A, Jiménez-Perepérez JA, Masana L and Turner PR. Low-density lipoprotein metabolism in rats treated with cyclosporine. Metabolism 1993;42:678-83.

180. Rayyes OA, Wallmark A and Florén C-H. Cyclosporine inhibits catabolism of low-density lipoproteins in HepG2 cells by about 25\%. Hepatology 1996;24:613-9.

181. Winegar DA, Salisbury JA, Sundseth SS and Hawke RL. Effects of cyclosporin on cholesterol 27-hydroxylation and LDL receptor activity in HepG2 cells. J Lipid Res 1996;37:179-91.

182. Azrolan N, Brown CD, Thomas L, Hayek T, Zhao ZH, Roberts KG, Scheiner C and Friedman EA. Cyclosporin A has divergent effects on plasma LDL cholesterol (LDL-C) and lipoprotein (a) [lp(a)] levels in renal transplant recipients. Arterioscler Thromb 1994;14:1393-8.

183. Apanay DC, Neylan JF, Ragab MS and Sgoutas DS. Cyclosporin increases the oxidizability of low-density lipoproteins in renal transplant recipients. Transplantation 1994;58:663-9.

184. Venkiteswaran K, Sgoutas DS, Santanam N and Neylan JF. Tacrolimus, cyclosporine and plasma lipoproteins in renal transplant recipients. Transpl Int 2001;14:405-10.

185. Devaraj S, Li DJ, Vazquez M and Jialal I. Cyclosporin A does not increase the oxidative susceptibility of low density lipoprotein in vitro. Free Rad Biol Med 1999;26:1064-8.

186. Claesson K, Mayer AD, Squifflet J-P, Grabensee B, Eigler FW, Behrend M, Vanrenterghem Y, Hooff J van, Morales JM, Johnson RWG, Buchholz B, Land W, Forsythe JLR, Neumayer HH, Ericzon B-G and Mühlbacher F. Lipoprotein patterns in renal transplant patients: A comparison between FK 506 and cyclosporin A patients. Transplant Proc 1998;30:1292-4.

187. Hohage H, Arlt M, Brückner D, Dietl KH, Zidek W and Spieker S. Effects of cyclosporin A and FK 506 on lipid metabolism and fibrinogen in kidney transplant recipients. Clin Transplant 1997;11:225-30.

188. McCune TR, Thacker LR II, Peters TG, Mulloy L, Rohr MS, Adams PA, Yium J, Light JA, Pruett T, Gaber AO, Selman SH, Jonsson J, Hayes JM, Wright FH jr, Armata T, Blanton J and Burdick JF. Effects of tacrolimus on hyperlipidemia after successful renal transplantation. A Southeastern Organ Procurement Foundation Multicenter Clinical Study. Transplantation 1998;65:87-92.

189. Artz MA, Boots JMM, Ligtenberg G, Roodnat JI, Christiaans MHL, Hené RJ, Blom HJ, Demacker PNM and Hilbrands LB. Randomized conversion from cyclosporin to tacrolimus in renal transplant patients: Improved lipid profile and unchanged plasma homocystein levels. Transplant Proc 2002;34:1793-4. 
190. Varghese Z, Fernando RL, Turakhia G, Psimenou E, Fernando ON, Sweny P, Powis SH and Moorhead JF. Calcineurin inhibitors enhance low-density lipoprotein oxidation in transplant patients. Kidney Int 1999;56(S71):S137-40.

191. Varghese Z, Fernando R, Turakhia G, Psimenou E, Brunton C, Fernando ON, Davenport A, Burns A, Sweny P, Powis SH and Moorhead JF. Oxidizability of low-density lipoproteins from neoral and tacrolimus-treated renal transplant patients. Transplant Proc 1998;30:2043-6.

192. Morena M, Vela C, Garrigue V, Catanzano J, Descomps B, Cristol JP and Mourad G. Lowdensity lipoprotein composition and oxidation are not influenced by calcineurin inhibitors in renal transplant patients. Transplant Proc 2000;32:2785-6.

193. Murgia MG, Jordan S and Kahan BD. The side effect profile of sirolimus: a phase I study in quiescent cyclopsorine-prednisone-treated renal transplant patients. Kidney Int 1996;49: 209-16.

194. Kahan BD, Podbielski J, Napoli KL, Katz SM, Meier-Kriesche H-U and Buren CT van. Immunosuppressive effects and safety of a sirolimus/cyclosporine combination regimen for renal transplantation. Transplantation 1998;66:1040-6.

195. Kahan BD, Napoli KL, Kelly PA, Podbielski J, Hussein I, Urbauer DL, Katz SH and Buren CT van. Therapeutic drug monitoring of sirolimus: correlations with efficacy and toxicity. Clin Transplant 2000;14:97-109.

196. Morrisett JD, Abdel-Fattah G and Kahan BD. Sirolimus changes lipid concentrations and lipoprotein metabolism in kidney transplant recipients. Transplant Proc 2003;35(S3A):143S-50.

197. Morrisett JD, Abdel-Fattah G, Hoogeveen R, Mitchell E, Ballantyne CM, Pownall HJ, Opekun AR, Jaffe JS, Oppermann S and Kahan BD. Effects of sirolimus on plasma lipids, lipoprotein levels, and fatty acid metabolism in renal transplant patients. J Lipid Res 2002;43:1170-80.

198. Kreis H, Cisterne J-M, Land W, Wramner L, Squifflet J-P, Abramowicz D, Campistol JM, Morales J-M, Grinyo J-M, Mourad G, Berthoux F-C, Brattström C, Lebranchu Y and Vialtel P for the Sirolimus European Renal Transplant Study Group. Sirolimus in association with mycophenolate mofetil induction for the prevention of acute graft rejection in renal allograft recipients. Transplantation 2000;69:1252-60.

199. Hooff JP van, Squifflet JP, Wlodarczyk Z, Vanrenterghem Y and Paczek L. A prospective randomized multicenter study of tacrolimus in combination with sirolimus in renal-transplant recipients. Transplantation 2003;75:1934-9.

200. Hoogeveen RC, Ballantyne CM, Pownall HJ, Opekun AR, Hachey DL, Jaffe JS, Oppermann S, Kahan BD and Morrisett JD. Effect of sirolimus on the metabolism of apoB100-containing lipoproteins in renal transplant patients. Transplantation 2001;72:1244-50.

201. Shapiro R, Jordan ML, Scantlebury VP, Vivas C, Marsh JW, McCauley J, Johnston J, Randhawa P, Irish W, Gritsch HA, Naraghi R, Hakala TR, Fung JJ and Starzl TE. A prospective, randomized trial of tacrolimus/prednisone versus tacrolimus/prednisone/ mycophenolate mofetil in renal transplant recipients. Transplantation 1999;67:411-5.

202. Isoniemi H, Tikkanen MJ, Ahonen J and Häyry P. Comparison of lipid and lipoprotein profiles in blood using double and triple immunosuppressive drug combinations. Transpl Int 1991;4: 130-5.

203. Boudreaux JP, McHugh L, Canafax DM, Ascher N, Sutherland DE, Payne W, Simmons RL, Najarian JS and Fryd DS. The impact of cyclosporine and combination immunosuppression on the incidence of posttransplant diabetes in renal allograft recipients. Transplantation 1987;44:376-81. 
204. Roth D, Milgrom M, Esquenazi V, Fuller L, Burke G and Miller J. Posttransplant hyperglycemia. Increased incidence in cyclosporine-treated renal allograft recipients. Transplantation 1989;47:278-81.

205. Pagano G, Bruno A, Cavallo-Perin P, Cesco L and Imbimbo B. Glucose intolerance after short-term administration of corticosteroids in healthy subjects. Prednisone, deflazacort, and betamethasone. Arch Intern Med 1989;149:1098-101.

206. Veenstra DL, Best JH, Hornberger J, Sullivan SD and Hricik DE. Incidence and long-term cost of steroid-related side effects after renal transplantation. Am J Kidney Dis 1999;33:829-39.

207. Hricik DE, Bartucci MR, Moir EJ, Mayes JT and Schulak JA. Effects of steroid withdrawal on posttransplant diabetes mellitus in cyclosporine-treated renal transplant recipients. Transplantation 1991;51:374-7.

208. Hollander AAMJ, Hené RJ, Hermans J, Es LA van and Woude FJ van der. Late prednisone withdrawal in cyclosporine-treated kidney transplant patients: a randomized study. J Am Soc Nephrol 1997;8:294-301.

209. Boots JMM, Christiaans MHL, Duijnhoven EM van, Suylen R-J van and Hooff JP van. Early steroid withdrawal in renal transplantation with tacrolimus dual therapy: A pilot study. Transplantation 2002;74:1703-9.

210. Fernandez LA, Lehmann R, Luzi L, Battezati A, Angelico MC, Ricordi C, Tzakis A and Alejandro R. The effects of maintenance doses of FK506 versus cyclosporin A on glucose and lipid metabolism after orthotopic liver transplantation. Transplantation 1999;68:1532-41.

211. Yamamoto H, Akazawa S, Yamaguchi Y, Yokota A, Yamasaki H, Nakanishi T, Tahara D, Matsuya F, Saito Y and Nagataki S. Effects of cyclosporin A and low dosages of steroid on posttransplantation diabetes in kidney transplant recipients. Diabetes Care 1991;14:867-70.

212. Nam JH, Mun JI, Kim SI, Kang SW, Choi KH, Park K, Ahn CW, Cha BS, Song YD, Lim SK, Kim KR, Lee HC and Huh KB. B-cell dysfunction rather than insulin resistance is the main contributing factor for the development of postrenal transplantation diabetes mellitus. Transplantation 2001;71:1417-23.

213. Robertson RP. Cyclosporin-induced inhibition of insulin secretion in isolated rat islets and HIT cells. Diabetes 1986;35:1016-9.

214. Gillison SL, Bartlett ST and Curry DL. Inhibition by cyclosporine of insulin secretion--a beta cell-specific alteration of islet tissue function. Transplantation 1991;52:890-5.

215. Shapiro R, Jordan M, Scantlebury V, Fung J, Jensen C, Tzakis A, McCauley J, Carroll P, Ricordi C, Demetris AJ, Mitchell S, Jain A, Iwaki Y, Kobayashi M, Reyes J, Todo S, Hakala TR, Simmons RL and Starzl TE. FK 506 in clinical kidney transplantation. Transplant Proc 1991;23:3065-7.

216. Shapiro R, Jordan ML, Scantlebury VP, Vivas C, Fung JJ, McCauley J, Randhawa P, Demetris AJ, Irish W, Mitchell S, Hakala TR, Simmons RL and Starzl TE. A prospective randomized trial of FK506-based immunosuppression after renal transplantation. Transplantation 1995; 59:485-90.

217. Boots JMM, Duijnhoven EM, Christiaans MHL, Nieman FHM, Suylen RJ van and Hooff JP van. Single center experience with tacrolimus versus cyclosporin-Neoral in renal transplant recipients. Transpl Int 2001;14:370-83.

218. First MR, Gerber DA, Hariharan S, Kaufman DB and Shapiro R. Posttransplant diabetes mellitus in kidney allograft recipients: incidence, risk factors, and management. Transplantation 2002;73:379-86. 
219. Duijnhoven EM van, Boots JMM, Christiaans MHL, Wolffenbuttel BHR and Hooff JP van. Influence of tacrolimus on glucose metabolism before and after renal transplantation: a prospective study. J Am Soc Nephrol 2001;12:583-8.

220. Duijnhoven EM van, Christiaans MHL, Boots JMM, Nieman FHR, Wolffenbuttel BHR and Hooff JP van. Glucose metabolism in the first 3 years after renal transplantation in patients on tacrolimus versus cyclosporine-based immunosuppression. J Am Soc Nephrol 2002;13:213-20.

221. Strumph P, Kirsch D, Gooding W and Carroll P. The effect of FK506 on glycemic response as assessed by the hyperglycemic clamp technique. Transplantation 1995;60:147-51.

222. Filler G, Neuschultz I, Vollmer I, Amendt P and Hocher B. Tacrolimus reversibly reduces insulin secretion in paediatric renal transplant recipients. Nephrol Dial Transplant 2000;15: $867-71$.

223. Dmitrewski J, KrentzAJ, Mayer AD, Buckels JA, Barnes AD, Smith J and Nattrass M. Metabolic and hormonal effects of tacrolimus (FK506) or cyclosporin immunosuppression following renal transplantation. Diabetes Obes Metab 2001;3:287-92.

224. Redmon JB, Olson LK, Armstrong MB, Greene MJ and Robertson RP. Effects of tacrolimus (FK506) on human insulin gene expression, insulin mRNA levels, and insulin secretion in HIT-T15 cells. J Clin Invest 1996;98:2786-93.

225. Tamura K, Fujimura T, Tsutsumi T, Nakamura K, Ogawa T, Atumaru C, Hirano Y, Ohara K, Ohtsuka $\mathrm{K}$ and Shimomura $\mathrm{K}$. Transcriptional inhibition of insulin by FK506 and positive involvement of FK506 binding protein-12 in pancreatic beta-cell. Transplantation 1995;59: 1606-13.

226. Goto T, Kino T, Hatanaka H, Nishiyama M, Okuhara M, Kohsaka M, Aoki H and Imanaka H. Discovery of FK-506, a novel immunosuppressant isolated from Streptomyces tsukubaensis. Transplant Proc 1987;19:4-8.

227. Meier-Kriesche H-U, Baliga R and Kaplan B. Decreased renal function is a strong risk factor for cardiovascular death after renal transplantation. Transplantation 2003;75:1291-5.

228. Abbott KC, Yuan CM, Taylor AJ, Cruess DF and Agodoa LYC. Early renal insufficiency and hospitalized heart disease after renal transplantation in the era of modern immunosuppression. J Am Soc Nephrol 2003;14:2358-65.

229. Curtis JJ, Luke RG, Dubovsky E, Diethelm AG, Whelchel JD and Jones P. Cyclosporin in therapeutic doses increases renal allograft vascular resistance. Lancet 1986;2:477-9.

230. Ishikawa A, Suzuki K and Fujita K. Mechanisms of Cyclosporine-induced nephrotoxicity. Transplant Proc 1999;31:1127-8.

231. Lanese DM and Conger JD. Effects of endothelin receptor antagonist on cyclosporine-induced vasoconstriction in isolated rat renal arterioles. J Clin Invest 1993;91:2144-9.

232. Kurihara H, Yoshizumi M, Sugiyama T, Takaku F, Yanagisawa M, Masaki T, Hamaoki M, Kato $\mathrm{H}$ and Yazaki Y. Transforming growth factor- $\beta$ stimulates the expression of endothelin mRNA by vascular endothelial cells. Biochem Biophys Res Com 1989;159:1435-40.

233. Chareandee C, Herman WH, Hricik DE and Simonson MS. Elevated endothelin-1 in tubular epithelium is associated with renal allograft rejection. Am J Kidney Dis 2000;36:541-9.

234. Simonson MS, Emancipator SN, Knauss T and Hricik DE. Elevated neointimal endothelin-1 in transplantation-associated arteriosclerosis of renal allograft recipients. Kidney Int 1998;54:960-71.

235. Simonson MS, Herman WH, Robinson A, Schulak J and Hricik DE. Inhibition of endothelinconverting enzyme attenuates transplant vasculopathy and rejection in rat cardiac allografts. Transplantation 1999;67:1542-7. 
236. Waiser J, Dell K, Böhler T, Dogu E, Gaedeke J, Budde K and Neumayer H-H. Cyclosporine A up-regulates the expression of TGF- $\beta 1$ and its receptors type I and type II in rat mesangial cells. Nephrol Dial Transplant 2002;17:1568-77.

237. Li B, Sehajpal PK, Khanna A, Vlassara H, Cerami A, Stenzel KH and Suthanthiran M. Differential regulation of transforming growth factor $B$ and interleukin 2 genes in human $T$ cells: Demonstration by usage of novel competitor DNA constructs in the quantitative polymerase chain reaction. J Exp Med 1991;174:1259-62.

238. Yamamoto T, Noble NA, Miller DE and Border WA. Sustained expression of TGF-B1 underlies development of progressive kidney fibrosis. Kidney Int 1994;45:916-27.

239. Sharma VK, Bologa RM, Xu G-P, Li B, Mouradian J, Wang J, Serur D, Rao V and Suthanthiran M. Intragraft TGF- $\beta_{1}$ mRNA: A correlate of interstitial fibrosis and chronic allograft nephropathy. Kidney Int 1996;49:1297-303.

240. Thomas SE, Andoh TF, Pichler RH, Shankland SJ, Couser WG, Bennett WM and Johnson RJ. Accelerated apoptosis characterizes cyclosporine-associated interstitial fibrosis. Kidney Int 1998;53:897-908.

241. Bakker RC, Kooten C van, Lagemaat-Paape ME van de, Daha MR and Paul LC. Renal tubular epithelial cell death and cyclosporin A. Nephrol Dial Transplant 2002;17:1181-8.

242. Halloran PF, Helms LMH, Kung L and Noujaim J. The temporal profile of calcineurin inhibition by cyclosporine in vivo. Transplantation 1999;68:1356-61.

243. Podder H, Stepkowski SM, Napoli KL, Clark J, Verani RR, Chou T-C and Kahan BD. Pharmacokinetic Interactions augment toxicities of sirolimus/cyclosporine combinations. J Am Soc Nephrol 2001;12:1059-71.

244. McAllister VC, Mahalati K, Peltekian KM, Fraser A and MacDonald AS. A clinical pharmacokinetic study of tacrolimus and sirolimus combination immunosuppression comparing simultaneous to separated administration. Ther Drug Monit 2002;24:346-50.

245. Smith KD, Wrenshall LE, Nicosia RF, Pichler R, Marsh CL, Alpers CE, Polissar N and Davis CL. Delayed graft function and cast nephropathy associated with tacrolimus plus rapamycin use. J Am Soc Nephrol 2003;14:1037-45.

246. Jain S, Bicknell GR and Nicholson ML. Tacrolimus has less fibrinogenic potential than cyclosporin A in a model of renal ischaemia-reperfusion injury. Br J Surg 2000;87:1563-8.

247. Bicknell GR, Shaw WJA, Pringle JH, Furness PN and Nicholson ML. Differential effects of cyclosporin and tacrolimus on the expression of fibrosis-associated genes in isolated glomeruli from renal transplants. Br J Surg 2000;87:1569-75.

248. Mohamed MAS, Robertson H, Booth TA, Balupuri S, Kirby JA and Talbot D. TGF- $B$ expression in renal transplant biopsies. A comparative study between cyclosporin-A and tacrolimus. Transplantation 2000;69:1002-5.

249. Wang T, Li B-Y, Danielson PD, Shah PC, Rockwell S, Lechleider RJ, Martin J, Manganaro T and Donahoe PK. The immunophilin FKBP12 functions as a common inhibitor of the TGF- $\beta$ family type 1 receptors. Cell 1996;86:435-44.

250. Wang T, Donahoe PK and Zervos AS. Specific interaction of type I receptors of the TGF- $\beta$ family with the immunophilin FKBP-12. Science 1994;265:674-6.

251. Khanna A, Cairns V and Hosenpud JD. Tacrolimus induces increased expression of transforming growth factor- $\beta 1$ in mammalian lymphoid as well as nonlymphoid cells. Transplantation 1999;67:614-9. 
252. Nakahama H, Fukunaga M, Kakihara M, Horio M, Fujiwara Y, Fukuhara Y, Ueda N, Orita Y and Kamada T. Comparative effect of cyclosporine A and FK-506 on endothelin secretion by a cultured renal cell line, LLC-PK1. J Cardiovasc Pharmacol 1991;17(S7):S172-3.

253. Baboolal K, Jones GA, Janezic A, Griffiths DR and Jurewicz WA. Molecular and structural consequences of early renal allograft injury. Kidney Int 2002;61:686-96.

254. Murphy GJ, Waller JR, Sandford RS, Furness PN and Nicholson ML. Randomized clinical trial of the effect of microemulsion cyclosporin and tacrolimus on renal allograft fibrosis. Br J Surg 2003;90:680-6.

255. Gjertson DW, Cecka JM and Terasaki PI. The relative effects of FK506 and cyclosporine on short- and long-term kidney graft survival. Transplantation 1995;60:1384-8.

256. Vincenti F, Jensik SC, Filo RS, Miller J and Pirsch J. A long-term comparison of tacrolimus (FK506) and cyclosporine in kidney transplantation: evidence for improved allograft survival at five years. Transplantation 2002;73:775-82.

257. Mayer AD and for the European Tacrolimus Multicentre Renal Study Group. Chronic rejection and graft half-life: Five year follow-up of the European Tacrolimus Multicenter Renal Study. Transplant Proc 2002;34:1491-2.

258. Kaplan B, Schold JD and Meier-Kriesche H-U. Long-term graft survival with Neoral and tacrolimus: A paired kidney analysis. J Am Soc Nephrol 2003;14:2980-4.

259. McCully KS. Vascular pathology of homocysteinemia: implications for the pathogenesis of arteriosclerosis. Am J Pathol 1969;56:111-28.

260. Mudd SH, Skovby F, Levy HL, Pettigrew KD, Wilcken B, Pyeritz RE, Andria G, Boers GH, Bromberg IL, Cerone R et al. The natural history of homocystinuria due to cystathionine betasynthase deficiency. Am J Hum Genet 1985;37:1-31.

261. Hankey GJ and Eikelboom JW. Homocysteine and vascular disease. Lancet 1999;354:407-13.

262. Refsum H, Ueland PM, Nygård O and Vollset SE. Homocysteine and cardiovascular disease. Annu Rev Med 1998;49:31-62.

263. Boushey CJ, Beresford SA, Omenn GS and Motulsky AG. A quantitative assessment of plasma homocysteine as a risk factor for vascular disease. Probable benefits of increasing folic acid intakes. J Am Med Assoc 1995;274:1049-57.

264. Frosst P, Blom HJ, Milos R, Goyette P, Sheppard CA, Matthews RG, Boers GJH, Heijer M den, Kluijtmans LAJ, Heuvel LP van den and Rozen R. A candidate genetic risk factor for vascular disease: a common mutation in methylenetetrahydrofolate reductase. Nat Genet 1995;10:111-3.

265. Verhoef P, Stampfer MJ, Buring JE, Gaziano JM, Allen RH, Stabler SP, Reynolds RD, Kok FJ, Hennekens $\mathrm{CH}$ and Willett WC. Homocysteine metabolism and risk of myocardial infarction: relation with vitamins B6, B12, and folate. Am J Epidemiol 1996;143:845-59.

266. Jacques PF, Bostom AG, Williams RR, Ellison RC, Eckfeldt JH, Rosenberg IH, Selhub J and Rozen R. Relation between folate status, a common mutation in methylenetetrahydrofolate reductase, and plasma homocysteine concentrations. Circulation 1996;93:7-9.

267. de Bree A, Verschuren WM, Blom HJ, Graaf-Hess A de, Trijbels FJ and Kromhout D. The homocysteine distribution: (mis)judging the burden. J Clin Epidemiol 2001;54:462-9.

268. Ueland PM, Refsum H, Beresford AA and Vollset SE. The controversy of homocysteine and cardiovascular risk. Am J Clin Nutr 2000;72:324-32.

269. Wald DS, Law M and Morris JK. Homocysteine and cardiovascular disease: evidence on causality from a meta-analysis. Br Med J 2002;325:1202. 
270. Arnadottir M, Hultberg B, Nilsson-Ehle P and Thysell H. The effect of reduced glomerular filtration rate on plasma total homocysteine concentration. Scan J Clin Lab Invest 1996;56: 41-6.

271. Wollesen F, Brattström L, Refsum H, Ueland PM, Berglund L and Berne C. Plasma total homocysteine and cysteine in relation to glomerular filtration rate in diabetes mellitus. Kidney Int 1999;55:1028-35.

272. Parsons DS, Reaveley DA, Pavitt DV and Brown EA. Relationship of renal function to homocysteine and lipoprotein(a) levels: The frequency of the combination of both risk factors in chronic renal impairment. Am J Kidney Dis 2002;40:916-23.

273. Stam F, Guldener C van, Schalkwijk CG, Wee PM ter, Donker AJM and Stehouwer CDA. Impaired renal function is associated with markers of endothelial dysfunction and increased inflammatory activity. Nephrol Dial Transplant 2003;18:892-8.

274. Guldener C van, Kulik W, Berger R, Dijkstra DA, Jakobs C, Reijngoud DJ, Donker AJM, Stehouwer CDA and Meer $\mathrm{K}$ de. Homocysteine and methionine metabolism in ESRD: a stable isotope study. Kidney Int 1999;56:1064-71.

275. Guldener C van, Donker AJM, Jakobs C, Teerlink T, Meer K de and Stehouwer CDA. No net renal extraction of homocysteine in fasting humans. Kidney Int 1998;54:166-9.

276. Sarnak MJ, Wang S-R, Beck GJ, Kusek JW, Selhub J, Greene T and Levey AS. Homocysteine, cysteine, and B vitamins as predictors of kidney disease progression. Am J Kidney Dis 2002;40: 932-9.

277. Krmar RT, Ferraris JR, Ramirez JA, Galarza CR, Waisman G, Janson JJ, Llapur CJ, Sorroche P, Legal S and Cámera MI. Hyperhomocysteinemia in stable pediatric, adolescents, and young adult renal transplant recipients. Transplantation 2001;71:1748-51.

278. Födinger M, Buchmayer H, Heinz G, Papagiannopoulos M, Kletzmayr J, RasoulRockenschaub S, Hörl WH and Sunder-Plassmann. Effect of MTHFR 1298A-C and MTHFR 677C-T genotypes on total homocysteine, folate, and vitamin $\mathrm{B}_{12}$ plasma concentrations in kidney graft recipients. J Am Soc Nephrol 2000;11:1918-25.

279. Beaulieu AJ, Gohh RY, Han H, Hakas D, Jacques PF, Selhub J and Bostom AG. Enhanced reduction of fasting total homocysteine levels with supraphysiological versus standard multivitamin dose folic acid supplementation in renal transplant recipients. Arterioscler Thromb Vasc Biol 1999;19:2918-21.

280. Fonseca I, Queirós J, Santos MJ, Mendonça D, Henriques AC, Sarmento AM, Santos AC, Guimarães $S$ and Pereira M. Hyperhomocysteinemia in renal transplantation: preliminary results. Transplant Proc 2000;32:2602-4.

281. Stein G, Müller A, Busch M, Fleck C and Sperschneider H. Homocysteine, its metabolites, and B-group vitamins in renal transplant patients. Kidney Int 2001;59(S78):S262-5.

282. Ducloux D, Motte G, Nguyen NH, Abdelfatah A, Gibey R and Chalopin J-M. Homocysteine, nutritional status and insulin in renal transplant recipients. Nephrol Dial Transplant 2002;17:1674-7.

283. Arnadottir M, Hultberg B, Wahlberg J, Fellström B and Dimény E. Serum total homocysteine concentration before and after renal transplantation. Kidney Int 1998;54:1380-4.

284. Huh W, Kim B, Kim SJ, Kim Y-G, Kim DJ, Cho JW and Oh H-Y. Changes of fasting plasma total homocysteine in the early phase of renal transplantation. Transplant Proc 2000;32:2811-3.

285. Arnadottir M, Hultberg B, Vladov V, Nilsson-Ehle P and Thysell H. Hyperhomocysteinemia in cyclosporine-treated renal transplant recipients. Transplantation 1996;61:509-12. 
286. Marcucci R, Fedi S, Brunelli T, Pepe G, Prisco D, Rosati A, Zanazzi M, Bertoni E, Abbate R and Salvadori M. High cysteine levels in renal transplant recipients. Relationship with hyperhomocysteinemia and 5,10-MTHFR polymorphism. Transplantation 2001;71:746-51.

287. Ducloux D, Motte G, Challier B, Gibey R and Chalopin J-M. Serum total homocysteine and cardiovascular disease occurrence in chronic, stable renal transplant recipients: a prospective study. J Am Soc Nephrol 2000;11:134-7.

288. Massy ZA, Chadefaux-Vekemans B, Chevalier A, Bader CA, Drueke TB, Legendre C, Lacour B, Kamoun P and Kreis H. Hyperhomocysteinaemia: a significant risk factor for cardiovascular disease in renal transplant recipients. Nephrol Dial Transplant 1994;9:1103-8.

289. Marcucci R, Zanazzi M, Bertoni E, Rosati A, Fedi S, Lenti M, Prisco D, Castellani S, Abbate R and Salvadori M. Vitamin supplementation reduces the progression of atherosclerosis in hyperhomocysteinemic renal-transplant recipients. Transplantation 2003;75:1551-5.

290. Massy ZA, Mamzer-Bruneel M-F, Chevalier A, Millet P, Helenon O, Chadefaux-Vekemans B, Legendre C, Bader C, Drüeke T, Lacour B and Kreis H. Carotid atherosclerosis in renal transplant recipients. Nephrol Dial Transplant 1998;13:1792-8.

291. Suwelack B, Gerhardt U, Witta J, Rahn KH and Hohage H. Effect of homocysteine on carotid intima-media thickness after renal transplantation. Clin Transplant 2000;14:555-60.

292. Dimény E, Hultberg B, Wahlberg J, Fellström B and Arnadottir M. Serum total homocysteine concentration does not predict outcome in renal transplant recipients. Clin Transplant 1998;12:563-8.

293. Hagen W, Födinger M, Heinz G, Buchmayer H, Hörl WH and Sunder-Plassmann. Effect of MTHFR genotypes and hyperhomocysteinemia on patient and graft survival in kidney transplant recipients. Kidney Int 2001;59(S78):S253-7.

294. Cole DEC, Ross HJ, Evrovski J, Langman LJ, Miner SES, Daly PA and Wong P-Y. Correlation between total homocysteine and cyclosporine concentrations in cardiac transplant recipients. Clin Chem 1998;44:2307-11.

295. Mor E, Helfmann L, Lustig S, Bar-Nathan N, Yussim A and Sela BA. Homocysteine levels among transplant recipients: effect of immunosuppressive protocols. Transplant Proc 2001;33:2945-6.

296. Ducloux D, Ruedin C, Gibey R, Vautrin P, Bresson-Vautrin C, Rebibou J-M and Chalopin JM. Prevalence, determinants, and clinical significance of hyperhomocyst(e)inemia in renaltransplant recipients. Nephrol Dial Transplant 1998;13:2890-3.

297. Ducloux D, Fournier V, Rebibou J-M, Bresson-Vautrin C, Gibey R and Chalopin J-M. Hyperhomocysteinemia in renal transplant recipients with and without cyclosporine. Clin Nephrol 1998;49:232-5.

298. Ignatescu MC, Födiger M, Kletzmayr J, Bieglmayer C, Hörl WH and Sunder-Plassmann G. Is there a role of cyclosporine A on total homocysteine export from human renal proximal tubular epithelial cells. Kidney Int 2001;59(S78):S258-61.

299. Fernández-Miranda C, Gómez P, Diaz-Rubio P, Estenoz J, Carillo JL, Andrés A and Morales JM. Plasma homocysteine levels in renal transplanted patients on cyclosporin or tacrolimus therapy: effect of treatment with folic acid. Clin Transplant 2000;14:110-4.

300. Mehra MR, Uber PA, Scott RL, Park MH and Milani RV. Effect of immunosuppressive regimen on novel markers of atherothrombosis in heart transplantation: homocysteine, Creactive protein, and mean platelet volume. Transplant Proc 2002;34:1866-8. 
301. Ignatescu MC, Kletzmayr J, Födiger M, Bieglmayer C, Hörl WH and Sunder-Plassmann G. Influence of mycophenolic acid and tacrolimus on homocysteine metabolism. Kidney Int 2002;61:1894-8.

302. Quiroga I, Morris-Stiff G, Baboo R, Darby CR, Lord RH and Jurewicz WA. Differential homocysteine levels in renal transplant patients receiving Neoral versus tacrolimus. Transplant Proc 2001;33:1209-10.

303. Hamsten A, Faire U de, Walldius G, Dahlen G, Szamosi A, Landou C, Blomback M and Wiman B. Plasminogen activator inhibitor in plasma: risk factor for recurrent myocardial infarction. Lancet 1987;2:3-9.

304. Thompson SG, Kienast J, Pyke SDM, Haverkate F and Loo JCW van de for the European Concerted Action on Thrombosis and Disabilities Angina Pectoris Study Group. Hemostatic factors and the risk of myocardial infarction or sudden death in patients with angina pectoris. N Engl J Med 1995;332:635-41.

305. Kannel WB, Wolf PA, Castelli WP and D'Agostino RB. Fibrinogen and risk of cardiovascular disease. The Framingham study. J Am Med Assoc 1987;258:1183-6.

306. Green D, Ruth KJ, Folsom AR and Liu K. Hemostatic factors in the Coronary Artery Risk Development in Young Adults (Cardia) Study. Arterioscler Thromb 1994;14:686-93.

307. Heinrich J, Schulte H, Schonfeld R, Kohler E and Assmann G. Association of variables of coagulation, fibrinolysis and acute-phase with atherosclerosis in coronary and peripheral arteries and those arteries supplying the brain. Thromb Haemost 1995;73:374-9.

308. Heinrich J, Balleisen L, Schulte H, Assman G and Loo J van de. Fibrinogen and factor VII in the prediction of coronary risk. Results from the PROCAM study in healthy men. Arterioscler Thromb 1994;14:54-9.

309. Juhan-Vague I, Roul C, Alessi MC, Ardissone JP, Heim M and Vague P. Increased plasminogen activator inhibitor activity in non insulin dependent diabetic patients--relationship with plasma insulin. Thromb Haemost 1989;61:370-3.

310. Juhan-Vague I, Alessi MC and Vague P. Increased plasma plasminogen activator inhibitor 1 levels. A possible link between insulin resistance and atherothrombosis. Diabetologia 1991;34:457-62.

311. Segarra A, Chacón P, Martinez-Eyarre C, Argelaguer X, Vila J, Ruiz P, Fort J, Bartolomé J, Camps J, Moliner E, Pelegrí A, Marco F, Olmos A and Piera L. Circulating levels of plasminogen activator inhibitor type-1, tissue plasminogen activator, and thrombomodulin in hemodialysis patients: biochemical correlations and role as independent predictors of coronary artery stenosis. J Am Soc Nephrol 2001;12:1255-63.

312. Labarrere CA, Pitts D, Nelson DR and Faulk WP. Vascular tissue plasminogen activator and the development of coronary artery disease in heart-transplant recipients. $\mathrm{N}$ Engl J Med 1995;333:1111-6.

313. Patrassi GM, Sartori MT, Rigotti P, Landro D di, Theodoridis P, Fioretti M, Capalbo M, Saggiorato G, Boeri G and Girolami A. Reduced fibrinolytic potential one year after kidney transplantation. Relationship to long-term steroid treatment. Transplantation 1995;59:1416-20.

314. Huser B, Lammle B, Landmann J, Tran TH, Oberholzer M, Marbet GA, Thiel G, Mihatsch MJ and Duckert F. Von Willebrand factor and factor VIII in renal transplant recipients under immunosuppression with cyclosporine and steroids. Sequential measurements over 4 months in 17 patients. Clin Nephrol 1990;34:214-22.

315. Patrassi GM, Dal Bo Zanon R, Boscaro M, Martinelli S and Girolami A. Further studies on the hypercoagulable state of patients with Cushing's syndrome. Thromb Haemost 1985;54:518-20. 
316. Dal Bo Zanon R, Fornasiero L, Boscaro M, Cappellato G, Fabris F and Girolami A. Increased factor VIII associated activities in Cushing's syndrome: a probable hypercoagulable state. Thromb Haemost 1982;47:116-117.

317. Patrassi GM, Sartori MT, Viero ML, Scarano L, Boscaro M and Girolami A. The fibrinolytic potential in patients with Cushing's disease: a clue to their hypercoagulable state. Blood Coagul Fibrinolysis 1992;3:789-93.

318. Patrassi GM, Sartori MT, Livi U, Casonato A, Danesin C, Vettore S and Girolami A. Impairment of fibrinolytic potential in long-term steroid treatment after heart transplantation. Transplantation 1997;64:1610-4.

319. Sartori MT, Maurizio PG, Sara P, Ugolino L, Annalisa A, Panagiotis T, Massimo F and Antonio G. Relation between long-term steroid treatment after heart transplantation, hypofibrinolysis and myocardial microthrombi generation. J Heart Lung Transplant 1999;18:693-700.

320. Sartori MT, Patrassi GM, Rigotti P, Marchini F, Fioretti M, Spiezia L and Girolami A. Improved fibrinolytic capacity after withdrawal of steroid immunosuppression in renal transplant recipients. Transplantation 2000;69:2116-21.

321. Sartori MT, Rigotti P, Marchini F, Spiezia L, Baldan N, Furian L, Varvarikis C and Girolami A. Plasma fibrinolytic capacity in renal transplant recipients: effect of steroid-free immunosuppression therapy. Transplantation 2003;75:994-8.

322. Laug WE. Glucocorticoids inhibit plasminogen activator by endothelial cells. Thromb Haemost 1983;50:888-92.

323. Huang LQ, Whitworth JA and Chesterman CN. Effects of cyclosporin A and dexamethasone on haemostatic and vasoactive functions of vascular endothelial cells. Blood Coagul Fibrinolysis 1995;6:438-45.

324. Pandit HB and Spillert CR. Effect of methylprednisolone on coagulation. J Natl Med Assoc 1999;91:453-6.

325. Levi M, Wilmink J, Büller HR, Surachno J and Cate JW ten. Impaired fibrinolysis in cyclosporine-treated renal transplant patients. Analysis of the defect and beneficial effect of fish-oil. Transplantation 1992;54:978-83.

326. Malyszko J, Malyszko JS, Pawlak K and Mysliwiec M. The coagulo-lytic system and endothelial function in cyclosporine-treated kidney allograft recipients. Transplantation 1996;62:828-30.

327. Dorpel MA van den, Man in 't Veld AJ, Levi M, Cate JW ten and Weimar W. Beneficial effects of conversion from cyclosporine to azathioprine on fibrinolysis in renal transplant recipients. Arterioscler Thromb Vasc Biol 1999;19:1555-8.

328. Vaziri ND, Ismail M, Martin DC and Gonzales E. Blood coagulation, fibrinolytic and inhibitory profiles in renal transplant recipients: comparison of cyclosporine and azathioprine. Int J Artif Organs 1992;15:365-9.

329. Murphy BG, Yong A, Brown JH and McNamee PT. Effect of immunosuppressive drug regime on cardiovascular risk profile following kidney transplantation. Atherosclerosis 1995;116:241-5.

330. Schrama YC, Dam T van, Fijnheer R, Hené RJ, Groot P de and Rabelink TJ. Cyclosporine is associated with endothelial dysfunction but not with platelet activation in renal transplantation. Neth J Med 2001;59:6-15. 
331. Morishita E, Nakao S, Asakura H, Jokaji H, Saito M, Uotani C, Kumabashiri I, Yamazaki M, Yoshida T, Takemoto K, Aoshima K, Hashimoto T and Matsuda T. Hypercoagulability and high lipoprotein(a) levels in patients with aplastic anemia receiving cyclosporine. Blood Coagul Fibrinolysis 1996;7:609-14.

332. Vanrenterghem Y, Roels L, Lerut T, Gruwez J, Michielsen P, Gresele P, Deckmyn H, Colucci $\mathrm{M}$, Arnout J and Vermylen J. Thromboembolic complications and haemostatic changes in cyclosporin-treated cadaveric kidney allograft recipients. Lancet 1985;1:999-1002.

333. Gruber SA, Pescovitz MD, Simmons RL, Najarian JS, Ascher NL, Payne WD, Sutherland DE and Fryd DS. Thromboembolic complications in renal allograft recipients. A report from the prospective randomized study of cyclopsorine versus azathioprine-antilymphocyte globulin. Transplantation 1987;44:775-8.

334. Baker LR, Tucker B and Kovacs IB. Enhanced in vitro hemostasis and reduced thrombolysis in cyclosporine-treated renal transplant recipients. Transplantation 1990;49:905-9.

335. Collins P, Wilkie M, Razak K, Abbot S, Harley S, Bax C, Zaidi M, Blake D, Cunningham J and Newland A. Cyclosporine and cremaphor modulate von Willebrand factor release from cultured human endothelial cells. Transplantation 1993;56:1218-23.

336. Kasiske BL, Chakkera HA, Louis TA and Ma JZ. A meta-analysis of immunosuppression withdrawal trials in renal transplantation. J Am Soc Nephrol 2000;11:1910-7.

337. Birkeland SA and Hamilton-Dutoit S. Is posttransplant lymphoproliferative disorder (PTLD) caused by any specific immunosuppressive drug or by the transplantation per se? Transplantation 2003;76:984-8.

338. Zanker B, Schneeberger H, Rothenpieler U, Hillebrand G, Illner W-D, Theodorakis I, Stangl $\mathrm{M}$ and Land W. Mycophenolate mofetil-based, cyclosporine-free induction and maintenance immunosuppression. First-3-months analysis of efficacy and safety in two cohorts of renal allograft recipients. Transplantation 1998;66:44-9.

339. Theodorakis J, Schneeberger H, Illner W-D, Stangl M, Zanker B and Land W. Nephrotoxicityfree, mycophenolate-based induction/maintenance immunosuppression in elderly recipients of renal allografts from elderly cadaveric donors. Transplant Proc 2000;32(S1A):9S-11.

340. Gregory CR, Huie P, Billingham ME and Morris RE. Rapamycin inhibits arterial intimal thickening caused by both alloimmune and mechanical injury. Transplantation 1993;55: 1409-18.

341. Cao W, Mohacsi P, Shorthouse R, Pratt R and Morris RE. Effects of rapamycin on growth factor-stimulated vascular smooth muscle cell DNA synthesis. Transplantation 1995;59:390-5.

342. Ikonen TS, Gummert JF, Hayase M, Honda Y, Hausen B, Christians U, Berry GJ, Yock PG and Morris RE. Sirolimus (rapamycin) halts and reverses progression of allograft vascular disease in non-human primates. Transplantation 2000;70:969-75.

343. Mancini D, Pinney S, Burkhoff D, LaManca J, Itescu S, Burke E, Edwards N, Oz M and Marks AR. Use of rapamycin slows progression of cardiac transplantation vasculopathy. Circulation 2003;108:48-53.

344. Morice M-C, Serruys PW, Sousa JE, Fajadet J, Ban Hayashi E, Perin M, Colombo A, Schuler G, Barragan P, Guagliumi G, Molnàr F and Falotico R for the RAVEL Study Group. A randomized comparison of a sirolimus-eluting stent with a standard stent for coronary revascularization. N Engl J Med 2002;346:1773-80. 
345. Moses JW, Leon MB, Popma JJ, Fitzgerald PJ, Holmes DR, O'Shaughnessy C, Caputo RP, Kereiakes DJ, Williams DO, Teirstein PS, Jaeger JL and Kuntz RE for the SIRIUS Investigators. Sirolimus-eluting stents versus standard stents in patients with stenosis in a native coronary artery. N Engl J Med 2003;349:1315-23.

346. Schofer J, Schlüter M, Gershlick AH, Wijns W, Garcia E, Schampaert E and Breithardt G for the E-SIRIUS Investigators. Sirolimus-eluting stents for treatment of patients with long atherosclerotic lesions in small coronary arteries: double-blind, randomised controlled trial (ESIRIUS). Lancet 2003;362:1093-9.

347. Morelon E, Stern M and Kreis H. Interstitial pneumonitis associated with sirolimus therapy in renal-transplant recipients. N Engl J Med 2000;343:225-6.

348. Morelon E, Stern M, Israël-Biet D, Corréas J-M, Danel C, Mamzer-Bruneel M-F, Peraldi M-N and Kreis $\mathrm{H}$. Characteristics of sirolimus-associated interstitial pneumonitis in renal transplant patients. Transplantation 2001;72:787-90.

349. Luan FL, Ding R, Sharma VK, Chon WJ, Lagman M and Suthanthiran M. Rapamycin is an effective inhibitor of human renal cancer metastasis. Kidney Int 2003;63:917-26.

350. Luan FL, Hojo M, Maluccio M, Yamaji K and Suthanthiran M. Rapamycin blocks tumor progression: Unlinking immunosuppression from antitumor efficacy. Transplantation 2002;73:1565-72.

351. Law BK, Chytil A, Dumont N, Hamilton EG, Waltner-Law ME, Aakre ME, Covington C and Moses HL. Rapamycin potentiates transforming growth factor $B$-induced growth arrest in nontransformed, oncogene-transformed, and human cancer cells. Mol Cell Biol 2002;22: 8184-98.

352. Guba M, Breitenbuch P von, Steinbauer M, Koehl G, Flegel S, Hornung M, Bruns CJ, Zuelke C, Farkas S, Anthuber M, Jauch K-W and Geissler EK. Rapamycin inhibits primary and metastatic tumor growth by antiangiogenesis: involvement of vascular endothelial growth factor. Nat Med 2002;8:128-35.

353. Muthukkumar S, Ramesh TM and Bondada S. Rapamycin, a potent immunosuppressive drug, causes programmed cell death in B lymphoma cells. Transplantation 1995;60:264-70.

354. Nepomuceno RR, Balatoni CE, Natkunam Y, Snow AL, Krams SM and Martinez OM. Rapamycin inhibits the interleukin 10 signal transduction pathway and the growth of Epstein Barr Virus B-cell lymphomas. Cancer Res 2003;63:4472-80.

355. Johnson C, Ahsan N, Gonwa T, Halloran P, Stegall M, Hardy M, Metzger R, Shield III C, Rocher L, Scandling J, Sorensen J, Mulloy L, Light J, Corwin C, Danovitch G, Wachs M, VanVeldhuisen P, Salm K, Tolzman D and Fitzsimmons WE. Randomized trial of tacrolimus (Prograf) in combination with azathioprine or mycophenolate mofetil versus cyclosporin (Neoral) with mycophenolate mofetil after cadaveric kidney transplantation. Transplantation 2000;69:834-41.

356. Gelder T van, Meulen CG ter, Hené R, Weimar W and Hoitsma A. Oral ulcers in kidney transplant recipients treated with sirolimus and mycophenolate mofetil. Transplantation 2003;75:788-91. 
Chapter 10

General discussion 
Chapter 10 


\section{General discussion}

\section{Efficacy: Tacrolimus versus Cyclosporin-microemulsion}

In two randomized multicenter trials, tacrolimus (TAC)-based immunosuppression was superior to Cyclosporin A (CsA)-Sandimmune-based immunosuppression with regard to the prevention of acute rejections ${ }^{1,2}$. This was fully in line with the fact that, on a molar basis, TAC is up to 100 times more potent than CsA in blocking calcineurin-dependent interleukin- 2 production ${ }^{3}$. An additional explanation could be the inconsistent bioavailability of CsA-Sandimmune. However, in 1995, the formulation of CsA that had been used was replaced by a microemulsion formulatin $\left(\mathrm{Neoral}^{\mathbb{R}}\right)$, one with a better and more consistent bioavailability ${ }^{4}$. This formulation appeared to reduce the incidence of acute rejection with CsA-based immunosuppression ${ }^{5-7}$. At that time, it was not known whether, with regard to efficacy, TAC would have the same advantage over CsAmicroemulsion as it had over CsA-Sandimmune. An interim report of an ongoing trial showed a comparable incidence of acute rejections between the two drugs ${ }^{8}$. However, in that study, the target drug levels in the first week of TAC were relatively low $(5-15 \mathrm{ng} / \mathrm{ml})$. To prevent rejections, at least in combination with mycophenolate mofetil (MMF), initial TAC target trough levels should exceed $10 \mathrm{ng} / \mathrm{ml}^{9}$. In order to evaluate the efficacy of TAC versus CsA-microemulsion, a retrospective analysis was performed of all our patients treated with TAC or CsAmicroemulsion between 1995 and the last quarter of 1997, prior to the registration of TAC in the Netherlands. In Chapter 2, this study clearly showed that the ability of TAC to prevent both first and recurrent rejections was also higher than that of CsA-microemulsion ${ }^{1}$. This occurred despite the fact that the patients in the TAC group were partly selected for their higher immunological risk of acute rejection. Thus, the lower efficacy obtained with CsA-Sandimmune could be extended to CsA-microemulsion. Our findings were confirmed in prospective studies from both a single center ${ }^{11}$ and a large, multicenter trial ${ }^{12}$. Recently, a meta-analysis came to the same conclusion ${ }^{13}$.

Although the incidence of steroid-resistant rejection in our study was lower with TAC, this difference was not statistically significant with the number of patients included. In addition, the use of rabbit-anti thymocyte globulin ( $r$-ATG) was numerically reduced. In a much larger prospective multicenter trial, TAC-treated patients indeed suffered significantly less from steroid-resistant acute rejection ${ }^{12}$. This is important because steroid-resistant and vascular rejections, in particular, have been reported to be a major risk factor for the subsequent development of chronic rejection ${ }^{14}$. Moreover, r-ATG is expensive and its use is associated with a higher morbidity and mortality rate ${ }^{15}$. 
The ability to reverse ongoing steroid-resistant rejections by conversion to TAC was previously demonstrated for patients on a CsA-Sandimmune-based immunosuppressive protocol ${ }^{16-18}$. In Chapter 2, we also observed that grafts with (recurrent) rejections under CsA-microemulsion could be rescued by conversion to TAC.

Despite stable renal function, histological signs of rejection can frequently be observed. These so-called subclinical rejections precede and correlate with chronic allograft nephropathy (CAN) ${ }^{19-22}$. The incidence of subclinical rejections depends on the type of immunosuppression. With a combination of TAC and MMF, the incidence at three to six months post-transplantation is between $3 \%$ and $14 \%{ }^{20,23,24}$ versus $24-35 \%$ with the combination of CsA-microemulsion and $\mathrm{MMF}^{20,24}$. Persistent, subclinical, acute rejection at 1 year post-transplantation was almost absent with TAC and MMF, whereas it was present in $32 \%$ of patients treated using CsA-microemulsion and $\mathrm{MMF}^{20}$.

Nowadays, one is recommended to monitor CsA using $\mathrm{C}_{2}$-levels (two hours after dosing) instead of $\mathrm{C}_{0}$-levels (trough levels, 12 hours after dosing) due to the better correlation with total drug exposure, possibly leading to a reduction in the incidence of acute rejection ${ }^{25}$. However, it has been shown that regardless of $\mathrm{C}_{2}$ guided dosing of CsA-microemulsion, the incidence of subclinical rejections is still significantly higher in CsA-microemulsion-treated patients than in TAC-treated patients $^{26}$.

As mentioned previously, acute rejections, especially steroid-resistant and vascular rejections, are a risk factor for the development of $\mathrm{CAN}^{14,27-31}$. Despite a significant reduction in the rate of acute rejections, our study did not show any advantage of TAC on graft survival. This is in accordance with the findings of the main prospective trials, which also saw comparable graft survival with CsASandimmune ${ }^{1,2}$ and CsA-microemulsion ${ }^{12,32}$. There are two explanations for this. First, the study populations may have been too small and, second, the follow-up may have been too short. Danovitch computed that, with an improvement of $20 \%$ in acute rejection rate and an estimated $25 \%$ increased chance of graft loss at three years post-transplantation as a result of acute rejection, several thousands of patients would need to be followed for at least three years to have sufficient statistical power to prove an improvement in graft survival ${ }^{33}$. Such trials are extremely difficult to perform. To detect a 30\% reduction in the annual rate of late graft loss with $80 \%$ power, 1500 patients would need to be followed for five years $^{34}$.

However, in their retrospective analysis of the United States (US) Renal Database, Gjertson et al. ${ }^{35}$ observed that, in the long term, TAC-treated patients had a better projected graft survival than CsA-treated patients. Follow-up data from the US trial showed comparable five-year graft survival between the two groups on an intention-to-treat analysis. However, a substantial proportion of the CsA-treated 
patients were converted to TAC, mainly due to rejection. After censoring for these converted patients, it was shown that patients treated with TAC had a better graft survival ${ }^{36}$. The five-year results of the European trial showed a longer projected graft half-life for TAC-treated patients ${ }^{37}$. Jurewicz reported long-term follow-up data from the Welsh Transplant Research Group ${ }^{11}$. After six years of follow-up, TAC-treated patients had significantly improved graft survival compared to CsAmicroemulsion-treated patients, and the projected graft half-life increased 50\% from 10 to 15 years. Thus, evidence supports better preserved graft survival with TAC-based immunosuppression. Recently, in a meta-analysis of 30 trials with a total of 4102 patients, the positive effects of TAC on graft survival were confirmed. It was shown that TAC-treated patients had a 44\% reduced risk of graft loss at six months post-transplantation and a $29 \%$ reduced risk at three years post-transplantation ${ }^{13}$.

Thus far, as reflected in the results of our study presented in Chapter 2, patient survival has always been comparable between the two immunosuppressive agents. However, the two-year follow-up data from the TAC versus CsA-microemulsion multicenter trial showed a significantly lower mortality in the TAC group on an intention-to-treat analysis ${ }^{32}$. Again, significantly more CsA-microemulsion-treated patients had been converted to TAC, which might have affected the results. A summary of the relative efficacy of TAC- and CsA-microemulsion-based immunosuppression is shown in Figure 10.1.

\section{Tacrolimus Cyclosporin micro-emulsion}

Acute rejection

Steroid-resistant rejection

Recurrent rejection

Rescue of CNI-resistant acute rejection

Graft survival at 5 years

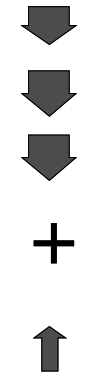

Figure 10.1 Comparison of the efficacy of tacrolimus-based immunosuppression versus cyclosporin-microemulsion-based immunosuppression. $\mathrm{CNI}=$ calcineurin inhibitor. 


\section{Steroid withdrawal and steroid avoidance}

Steroid withdrawal had already been attempted in the CsA era. Although it succeeded in many patients, it included an increased risk of rejection and subsequent graft failure ${ }^{38-42}$. This was also demonstrated in a meta-analysis of these trials ${ }^{43}$. In our experience, steroid withdrawal in the CsA era was possible by tailor-made immunosuppression in a selected group of low and intermediate immunological risk patients with no previous acute rejection.

The improved ability of TAC to prevent acute rejections made it possible to withdraw steroids without increasing the risk of rejections. The Pittsburgh group was the first to demonstrate the possibility of withdrawing steroids in about half of their TAC-treated patients ${ }^{44,45}$. However, they reported a 5\% risk of rejection after withdrawal of the steroids ${ }^{45}$. Our study (Chapter 2 ) shows that we were able to successfully withdraw steroids in $80 \%$ of the TAC-treated patients in contrast to $42 \%$ of the CsA-microemulsion-treated patients. No rejections occurred in the TAC group compared to nearly $10 \%$ of the weaned patients in the CsAmicroemulsion group. Furthermore, $70 \%$ could be successfully treated with TAC monotherapy ${ }^{10}$.

Rapid discontinuation of steroids implies a risk of adrenal insufficiency because steroids suppress the hypothalamic-pituitary-adrenal axis (HPA-axis) ${ }^{46-48}$. This risk has never been clearly established in the renal transplant population. In Chapter 3, we demonstrated that the HPA-axis is suppressed in about half of the patients and that the degree of suppression increases with the duration of the therapy. Despite this suppression, rapid discontinuation of $10 \mathrm{mg}$ prednisolone, which is the usual steroid maintenance dose in many transplant centers, can be safely performed after three months of therapy. After six months of therapy, however, mild symptoms that could be attributed to adrenal insufficiency were observed in three out of ten patients. In situations involving stress or surgery, this has to be taken into account. Similar results were obtained in a Swiss study with a similar steroid withdrawal protocol performed a mean three years after transplantation. In that study, symptoms of hypocorticism occurred in 30\% of the patients after steroid withdrawal ${ }^{49}$. In our patients, a baseline cortisol level and, in the case of a baseline level between 200 and $400 \mathrm{nmol} / \mathrm{l}$, a second level 30 minutes after the administration of $0.25 \mathrm{mg}$ synacthen (short synacthen test), had a negative predictive value of $80 \%$. Thus, this test can tell us for which patients the rapid discontinuation of steroids might be safe. Yet, because the short synacthen test has a positive predictive value of only $40 \%$, patients at risk cannot reliably be detected with it.

Both steroids and TAC are metabolized by the same cytochrome system (cytochrome P450, subtype 3A4)50,51. This may lead to interactions. Indeed, steroids were found to induce this system both in rats and humans ${ }^{50,52-55}$, although 
they were less potent than rifampicin ${ }^{54}$. In Chapter 4 , it is shown that cessation of steroids gives rise to an increase in TAC trough levels in most, but not all, patients. The degree of the increase depends on the strength of the steroid dose. These results were confirmed by the study reported by Hesselink et al. ${ }^{56}$. An increase in the area under the curve (AUC) after steroid cessation is compatible with a reduced metabolism of TAC after steroid cessation ${ }^{57}$. Since TAC influences GFR, an increase in TAC trough levels can mimic an episode of acute rejection after steroid withdrawal. On the other hand, steroids lead to an increase in costs because higher dosages of TAC are necessary to achieve the same trough levels.

In Chapter 2, we demonstrated that steroid withdrawal was successful in TACtreated patients after the initial three to six months post-transplantation. Grewal et al. ${ }^{58}$ were successful in stopping steroids as early as one week post-transplantation. In that study, the steroid dosages administered in the first week were remarkably high, much higher than those normally used, namely, almost $1500 \mathrm{mg}$ methylprednisolone for a patient of $70 \mathrm{~kg}$. Furthermore, due to the administration of additional immunosuppressive drugs like MMF, the real potency of the steroids and the additional effect on the prevention of acute rejections with TAC-based immunosuppression cannot be determined. Since many patients in our center were already successfully treated with TAC monotherapy as maintenance immunosuppression, it was postulated that it might also be possible to withdraw steroids early after transplantation in low and intermediate risk patients, provided adequate TAC trough levels had been achieved. In Chapter 5, it was shown that early cessation of steroids did not increase the incidence of acute rejections. On the contrary, the time to the first episode of acute rejection was even longer in the group that had their steroids withdrawn early ${ }^{59}$. The explanation for this unexpected observation lies most probably in the increase in TAC trough levels after the cessation of steroids, as discussed previously ${ }^{57}$.

The incidence of acute rejections in this study was about 30\%59. Remarkably, other European studies using TAC dual therapy with even higher dosages of the steroids resulted in similar rates of acute rejection ${ }^{60-62}$. In addition, a trial with TAC monotherapy once again showed an acute rejection rate of about $30 \%$. Therefore, it is unlikely that steroids contribute in a major way to the prevention of acute rejections in TAC-based immunosuppression when TAC levels are in the target range. Adequate TAC exposure is essential to prevent rejections. To achieve adequate TAC levels directly after transplantation, pre-transplant dosing may be useful. If, in addition, TAC levels are measured two hours after dosing patients can be detected who are at risk of underdosing ${ }^{64}$. Since TAC levels within the target range will not be reached in all patients, additional immunosuppressive agents may be necessary. 
DNA polymorphisms, especially for cytochrome P450 subtype 3A5, will result in substantial differences in TAC exposure ${ }^{65}$. Determination of this polymorphism is another possible way to adjust dosage beforehand.

Trials have recently been published showing excellent results with early steroid withdrawal ${ }^{66,67}$ or even successful transplantation without the use of steroids ${ }^{68,69}$. In contrast to our trial, additional immunosuppressive drugs were used to make the withdrawal of the steroids possible. In line with our results, steroid cessation did not result in a worse outcome.

Nowadays, an acute rejection rate of around 30\% is no longer acceptable. To reduce the rate of acute rejections, immunosuppressive drugs other than steroids need to be added. The combination of TAC and MMF or sirolimus (RAPA) reduces the rate of acute rejections to $15 \%{ }^{61,67,69,70}$ and $10 \%{ }^{71}$, respectively. Thus, in the early stage, only a small proportion (around 15\%) of the general transplant population will benefit from this additional immunosuppression. The majority $(70 \%)$ will experience only the side effects and will become overimmunosuppressed. It is not known what the long-term benefits of these additional agents are.

It is known that several categories of patients have an increased risk of acute rejection, namely, blacks, young patients, those who have antibodies to HLA antigens and, most importantly, those with mismatches in the HLA-system, especially HLA-DR $27,29,30,72-79$. In the future, the optimal degree of immunosuppression will have to be defined for each of these risk factors. The choice of immunosuppression will then be more tailor-made to the individual patient.

Steroid cessation in the CsA era resulted in an increased risk of graft failure due to chronic transplant nephropathy ${ }^{43}$. Thus far, the follow-up of TAC-treated patients after steroid withdrawal has been relatively limited, and firm data have not yet been published. In our study, no worse effects were seen on surrogate markers for the development of chronic transplant nephropathy (e.g., serum creatinine or creatinine clearance and proteinuria) at one year post-transplantation ${ }^{59}$. In the late 1990s, a large multicenter trial was performed in Europe to evaluate the effect of cessation of either steroids or MMF at three months post-transplantation from TAC-based triple therapy with steroids and MMF (Thomas trial) ${ }^{70}$. After three months, a small but non-significant increase in acute rejection was observed in the group that had their steroids withdrawn, but these data might be overestimated due to a possible increase in serum creatinine as a result of an increase in TAC trough levels after the withdrawal of steroids ${ }^{57}$. A three-year follow-up of the patients showed, in an intention-to-treat analysis, similar graft survival rates and a similar slope of the curve of serum creatinine in all groups. Thus, no negative effect of the cessation of the steroids on three-year graft survival could be 
observed $^{80}$. Recently, Matas et al. ${ }^{81}$ published their five-year single center experience of a protocol of prednisone-free maintenance immunosuppression from the second week post-transplantation onwards. In this study, graft outcome was comparable to that of historical controls on maintenance steroids.

Aside from possible, but as yet unproven, positive effects of steroids on the prevention of acute and chronic rejection, steroids have many undesirable side effects. They induce hypertension $42,67,82$, hyperlipidemia $42,67,82$, and diabetes mellitus $39,83,84$, all of which have been proven to impair graft survival. These side effects may, therefore, counteract the potentially (unproven) beneficial effects of steroids on the prevention of chronic rejection.

Outside the cardiovascular system, steroids induce weight gain and obesity, osteoporosis and avascular necrosis of the bone, atrophy of the skin and vessel wall, cataracts and glaucoma, myopathy and tendon ruptures, and psychiatric disorders ${ }^{83,94,95}$. As a result of these side effects, steroids substantially increase the costs of health care by $\$ 5300$ per patient per year ${ }^{83}$. The pros and cons of steroid therapy in TAC-based immunosuppression are presented in Table 10.1.

Table 10.1 The pros and cons of the use of steroids in tacrolimus-based immunosuppression in renal transplantation

\begin{tabular}{|c|c|}
\hline \multicolumn{2}{|c|}{ Steroids } \\
\hline Pro & Con \\
\hline Apoptotic effect on lymphocytes & Cardiovascular system \\
\hline induction therapy & endothelial dysfunction \\
\hline treatment of acute rejection & induction of hypertension \\
\hline & induction of hypercholesterolemia (total and LDL) \\
\hline ? Prevention of acute rejection & induction of hypertriglyceridemia \\
\hline ? Prevention of chronic rejection & $\begin{array}{l}\text { increase in insulin resistance and diabetes mellitus } \\
\text { impairment of fibrinolysis }\end{array}$ \\
\hline \multicolumn{2}{|l|}{ Increase in HDL-cholesterol } \\
\hline & $\begin{array}{l}\text { ? Chronic allograft nephropathy by cardiovascular side } \\
\text { effects }\end{array}$ \\
\hline & Outside the cardiovascular system \\
\hline & osteoporosis and avascular bone necrosis \\
\hline & atrophy of the skin and vessel wall \\
\hline & glaucoma and cataract \\
\hline & myopathy and tendon ruptures \\
\hline & psychiatric disorders \\
\hline & Enhancement of tacrolimus metabolism \\
\hline & Substantial increase in healthcosts \\
\hline
\end{tabular}




\section{Post-Transplant Diabetes mellitus}

In the main trials comparing TAC with $\mathrm{CsA}$, it was observed that the incidence of new-onset or post-transplant diabetes mellitus (PTDM) was significantly increased in TAC-treated patients ${ }^{1,2}$. The incidence in these patients depends on the definition used. As PTDM was defined as insulin dependency for more than 30 consecutive days, an incidence of around $20 \%$ was originally reported ${ }^{1,45}$. In a recent meta-analysis, the incidence of PTDM (insulin dependency $>30$ days) decreased but was still significantly higher in TAC-treated renal transplant recipients $(9.8 \%)$ than in CsA-treated recipients $(2.7 \%)^{96}$. This meta-analysis included publications from January 1992 until April 2002; more recent publications, in which the immunosuppressive protocol had been changed towards lower TAC target levels and diminishing or withdrawing steroids, were not included ${ }^{67,69}$. As shown by Pagano et al., steroids increase insulin resistance ${ }^{97}$. Recently a review on the mechanism of PTDM in TAC patients was published ${ }^{98}$. Patients who developed diabetes already had a subclinical impairment of glucose metabolism prior to transplantation ${ }^{99-101}$. Therefore, PTDM is not a separate entity, but rather is characterized by an underlying metabolic disorder uncovered by immunosuppression. In the study by van Duijnhoven et al., it was shown that TAC interferes with the secretion of insulin ${ }^{100}$. Other investigators reported an increase in insulin resistance ${ }^{102}$. Concomitant use of steroids may account for this observation. The real contribution of both TAC and steroids to glucose metabolism remained unclear.

In Chapter 6, first, the effect of steroid withdrawal and, second, the influence of TAC dose reduction on glucose metabolism were studied. It was shown that steroids increase insulin resistance and have no effect on insulin secretion whereas TAC impairs insulin secretion and has no effect on insulin resistance ${ }^{103}$. The effect of TAC on insulin secretion is fully in line with the results of other studies ${ }^{100,104-106 .}$ In vitro experiments showed that TAC dose dependently and completely reversibly inhibited insulin gene transcription starting after 24 hours, that TAC had no acute effects on insulin secretion, and that TAC did not affect glucose uptake due to insulin ${ }^{107,108}$. Both calcineurin and FK-binding protein (FKBP)-12 are present in pancreatic $\alpha$ and $\beta$-cell lines. Calcineurin is more prominent in $\alpha$-cells, unlike FKBP-12, which is very prominent in $\beta$-cells ${ }^{108}$. The inhibition of insulin secretion by TAC probably occurs along the same pathway as the blockade of IL-2 production in lymphocytes ${ }^{107,108}$. Given the high content of FKBP-12 and the high potency of TAC to block calcineurin, $\beta$-cells may be very sensitive to TAC.

Not only TAC, but also CsA has an effect on glucose metabolism. In liver transplant recipients treated with CsA, glucose tolerance was impaired compared to control patients ${ }^{104}$. Renal transplant patients using CsA and steroids had a higher incidence of PTDM than patients treated with azathioprine (AZA) and steroids ${ }^{109,110}$, even when steroid dosages were higher in the combination with 
$\mathrm{AZA}^{110}$. Reducing the dose of $\mathrm{CsA}$ reversed the diabetes in the majority of affected patients ${ }^{110}$. Glucose tolerance also improved after conversion from CsA to AZA ${ }^{111}$. In vitro experiments showed that CsA, like TAC, dose dependently inhibited insulin secretion ${ }^{112,113}$. An impaired pancreatic $\beta$-cell secretory function has also been reported in transplant patients ${ }^{101,104}$. In contrast, Sato et al. ${ }^{99}$ reported an increase in insulin-secreting capacity directly after transplantation in CsA-treated patients, whereas there was a slight decrease in insulin-secreting capacity in TAC-treated patients. These observations can be explained by an increase in insulin resistance by the concomitant use of steroids, which has to be counterbalanced by an increase in insulin-secreting capacity. The greater ability of TAC to block calcineurin ${ }^{3}$ and the high concentration of FKBP-12 108 may account for the fact that the necessary increase in insulin-secreting capacity was not observed with TAC. The same mechanism is responsible for the higher incidence of diabetes observed in TAC-treated patients.

In many patients, PTDM was reversible after the dose of TAC was reduced and the steroids withdrawn. In our study in chapter 2, more than half of the patients who developed diabetes mellitus were able to refrain from anti-diabetic drugs after the dose of TAC was reduced and the steroids withdrawn. Then, the incidence of diabetes in the maintenance phase after transplantation was comparable between TAC- and CsA-treated patients ${ }^{10}$. These results are fully in line with data from the United States reporting an incidence of PTDM of 5.7\% in the maintenance phase ${ }^{114}$. In another study by our group, it was shown that glucose metabolism did not differ between the two drugs after three months post-transplantation ${ }^{115}$.

Since both an increase in insulin resistance by steroids and a decrease in insulin secretory capacity by calcineurin blockers play a role in the development of PTDM, it is important to evaluate which of the two mechanisms is clinically more important. In Chapter 6, median $\mathrm{k}_{\mathrm{G}}$-values, resulting from both insulin secretion and resistance, increased after steroid withdrawal and remained stable after tapering the dose of TAC, suggesting that the impact of the steroids might be of greater importance (Figure 10.2). This conclusion is confirmed by the observation in Chapter 5 that a low incidence of PTDM was observed in the group that had their steroids withdrawn early ${ }^{59}$. Despite the use of high steroid dosages in the first week, also Grewal et al. reported a low incidence of diabetes in their study of early steroid cessation ${ }^{58}$. In addition, the cumulative steroid dose was significantly correlated to the development of PTDM ${ }^{99,116}$. More recently, low incidences of PTDM were observed with avoidance of steroids, thus providing more evidence that the increase in resistance is clinically more important ${ }^{67-69,81,117}$. Thus, steroids play the major role in the development of PTDM. Early withdrawal or avoidance of steroids is safe and will prevent the development of diabetes in many transplant patients. 


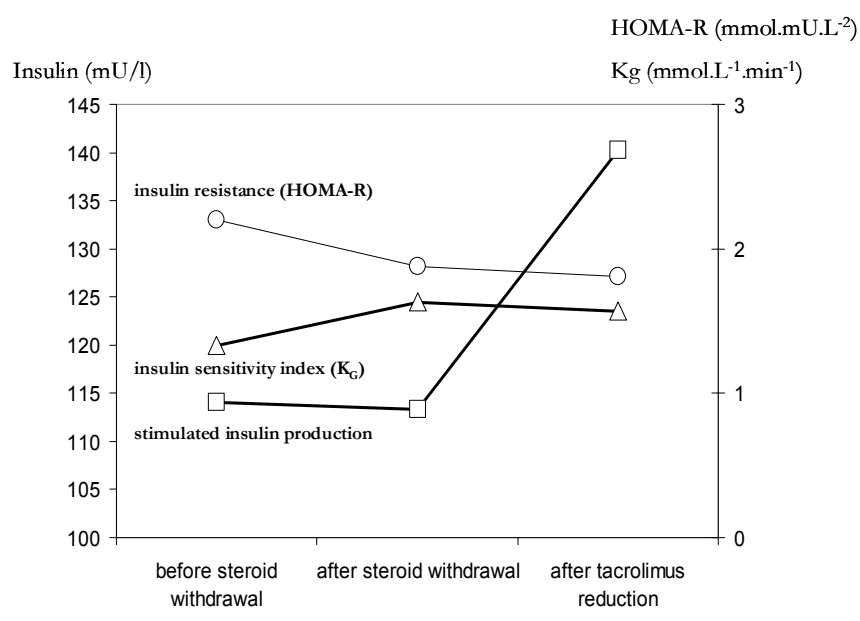

Figure 10.2 The effect of both steroid withdrawal and TAC trough level reduction on several parameters of glucose metabolism. HOMA-R=homeostasis model assessment= fasting glucose level $\mathrm{x}$ fasting insulin level/22.5. $\mathrm{K}_{\mathrm{G}}=$ glucose sensitivity index=linear regression of log-transformed glucose levels between 10 and 30 minutes after glucose administration in the intravenous glucose tolerance test (iv-GTT).

A substantial proportion of transplant candidates already have impaired glucose metabolism during dialysis ${ }^{100,101}$. These patients especially are at risk of developing diabetes after transplantation. This observation was confirmed in other studies ${ }^{99,118}$. The value of pre-transplant screening was demonstrated in a recent study in which a poor response to an oral glucose tolerance test (o-GTT) correlated strongly with the subsequent development of PTDM ${ }^{118}$. Therefore, it is recommended to screen future transplant candidates for an underlying prediabetic state, e.g., by performing an o-GTT. Patients identified as high risk may benefit from pre-transplant lifestyle intervention, such as exercise training and reducing obesity, to minimize the risk of diabetes mellitus type 2 (DM2) and PTDM. Lifestyle intervention has been shown to reduce the incidence of DM2 by more than $50 \%{ }^{119,120}$. Furthermore, dosing of the immunosuppressive agents can be adjusted so that it has a minimal effect on glucose metabolism, as discussed below.

Insulin resistance is recognized as being a major characteristic of the so-called metabolic syndrome, which is characterized by insulin resistance, diabetes mellitus, obesity (especially an increase in abdominal fat), hypertension, hyperlipidemia, and a poor cardiovascular outcome ${ }^{121}$. It has also been postulated that insulin resistance could be the leading cause of the recipient-related risk factors for 
chronic transplant nephropathy ${ }^{122}$. Indeed, in a cross-sectional study, the presence of the metabolic syndrome was associated with impaired renal allograft function beyond the first year post-transplantation. However, not all components of the metabolic syndrome contributed equally, and only systolic blood pressure and hypertriglyceridemia were independently associated in multivariate analyses ${ }^{123}$. Remarkably, many effects of the use of steroids resemble those of the metabolic syndrome. They induce insulin resistance, hyperlipidemia, hypertension, and increase the amount of abdominal fat.

Treatment of diabetes implies more than just lowering blood glucose levels. For both pre-diabetic and DM2 patients, a multifactorial approach is advocated that can reduce the burden of cardiovascular disease by about $50 \% 0^{124-126}$. Next to careful blood glucose regulation, this approach includes lifestyle intervention, strict blood pressure (target blood pressure $<125 / 75 \mathrm{mmHg}$ ) and cholesterol (target serum LDL-cholesterol $<2.6 \mathrm{mmol} / \mathrm{l}$ ) control. The latter two are the most realistic targets in reducing cardiovascular risk in DM2 $2^{124,127,128}$. However, the relative importance of glucose metabolism, blood pressure, and hyperlipidemia on cardiovascular mortality for renal transplant recipients is unknown. The importance of immunosuppressive agents with regard to these factors is depicted in Table 10.2.

Table 10.2 The effects of immunosuppressive agents on important factors for the management of diabetes mellitus.

\begin{tabular}{|c|c|c|c|c|c|c|}
\hline & Cyclosporin A & Tacrolimus & Steroids & Sirolimus & AZA & MMF \\
\hline Low rejection & - & + & - & - & - & - \\
\hline Steroid-free & $-/+$ & ++ & - & - & - & - \\
\hline Low lipids & - & + & - & - & + & + \\
\hline Low blood pressure & - & + & - & + & + & + \\
\hline Diabetes mellitus & + & +1 & ++ & $-/+2$ & - & - \\
\hline
\end{tabular}

Steroids, sirolimus, azathioprine (AZA) and mycophenolate mofetil (MMF) are used only in combination therapy. ${ }^{1}$ The effect of tacrolimus on the development of diabetes is dose-dependent and nearly absent with steroid avoidance; ${ }^{2}$ In a recent publication, it was shown that treatment with sirolimus might have an effect on insulin resistance, probably by inducing hypertriglyceridemia ${ }^{205}$.

For transplant patients, this multifactorial approach has to be extended to include the avoidance or minimization of steroids, both maintenance and boluses, because steroids induce diabetes, hypertension and, hypercholesterolemia, as discussed previously ${ }^{129}$. In addition, dosages of calcineurin blockers need to be strictly controlled at the lowest safe levels. During the first two weeks after transplantation, TAC trough levels should be kept between 15 and $20 \mathrm{ng} / \mathrm{ml}$, and 
during week three and four between 10 and $15 \mathrm{ng} / \mathrm{ml}$ to avoid acute rejection ${ }^{130}$. Thereafter, TAC trough levels may be reduced to below $10 \mathrm{ng} / \mathrm{ml}$. Our guidelines were recently confirmed and extended by a meta-analysis ${ }^{13}$. The authors showed that the risk of diabetes with TAC was lower when trough levels were below $10 \mathrm{ng} / \mathrm{ml}$, while the gain in graft function was optimal with these concentrations. If patients are converted to CsA in this period, which is often advocated, or to calcineurin-free immunosuppression, this might be counterproductive; a potentially reversible disease (PTDM) could be exchanged for the risk of a potentially irreversible event (acute rejection), which would endanger long-term graft survival. Acute rejection requires treatment with steroid boluses and this may even lead to worsening of PTDM. Moreover, such a conversion usually implies the maintenance or re-introduction of steroids to prevent rejection ${ }^{131}$. Such a conversion means changing a regimen with minor effects on lipids and blood pressure, as will be discussed in a following paragraph, for a regimen with major effects on lipids (steroids, CsA, sirolimus) and blood pressure (steroids, CsA). As discussed previously, strict blood pressure and lipid control are more realistic targets in reducing cardiovascular risk than strict blood glucose control in DM2 $2^{124,127,128}$.

When the risk of acute rejection is too high for early withdrawal, steroids may be reduced to $10 \mathrm{mg} /$ day at month 1 . After this period, steroids may be gradually withdrawn or, in patients at an unacceptably high risk of rejection, reduced to 5 $\mathrm{mg} /$ day. TAC trough levels should be reduced to $4-7 \mathrm{ng} / \mathrm{ml}$ at month $3{ }^{129}$. Drug monitoring must continue during corticosteroid taper and cessation because TAC exposure will increase at this time, as we have demonstrated in Chapter $4^{57}$.

In this period of dose optimisation, it may be necessary to improve glycemic control with insulin or oral anti-diabetic agents. However, such a treatment must be used with caution since PTDM may resolve, introducing the risk of hypoglycemia. These measures will result in a reversal of PTDM in the majority of the recipients. For those patients in whom impaired glycemic control does not resolve, the degree of insulin resistance should be determined. If the resistance is high, obesity or underlying infection is generally present. Of course, these factors have to be treated appropriately. Moreover, the use of newer anti-diabetic agents, like the thiazolidinediones, which are known to improve insulin resistance and have recently been shown to improve cardiovascular outcome, may be considered ${ }^{132-135}$. In patients with normal insulin resistance, the insulin output will be low. In such a situation, it is important to determine TAC blood levels two hours after dosing $\left(\mathrm{C}_{2}\right)$. Since exposure to TAC (AUC) in relation to its trough level may differ by a factor of two to three ${ }^{136}$, depending on the trough level, a relatively high exposure to TAC may be present. Further TAC dose reduction will then be the best option to manage the diabetes. A flow chart of the management of PTDM is presented in Figure 10.3. 
Generally, conversion after three to six months from TAC to CsA is of little value since the effect of TAC on glucose metabolism in this period is minor and comparable to that of $\mathrm{CsA}^{115}$, whereas conversion to calcineurin inhibitor-free immunosuppression poses an additional risk of acute rejection. As we showed in Chapters 7 and 8 , no significant increase in the incidence of diabetes occurred after conversion ${ }^{137,138}$. These data were recently confirmed and extended by Waid et al. ${ }^{139}$.

A recent study using iv-GTT showed that, before conversion, $40 \%$ of the nondiabetic CsA-treated transplant patients already had a disturbed glucose metabolism: $33 \%$ had a pre-diabetic and $7 \%$ a diabetic insulin sensitivity index. After conversion to TAC, no significant changes in the $\mathrm{k}_{\mathrm{G}}$ were observed ${ }^{140}$.

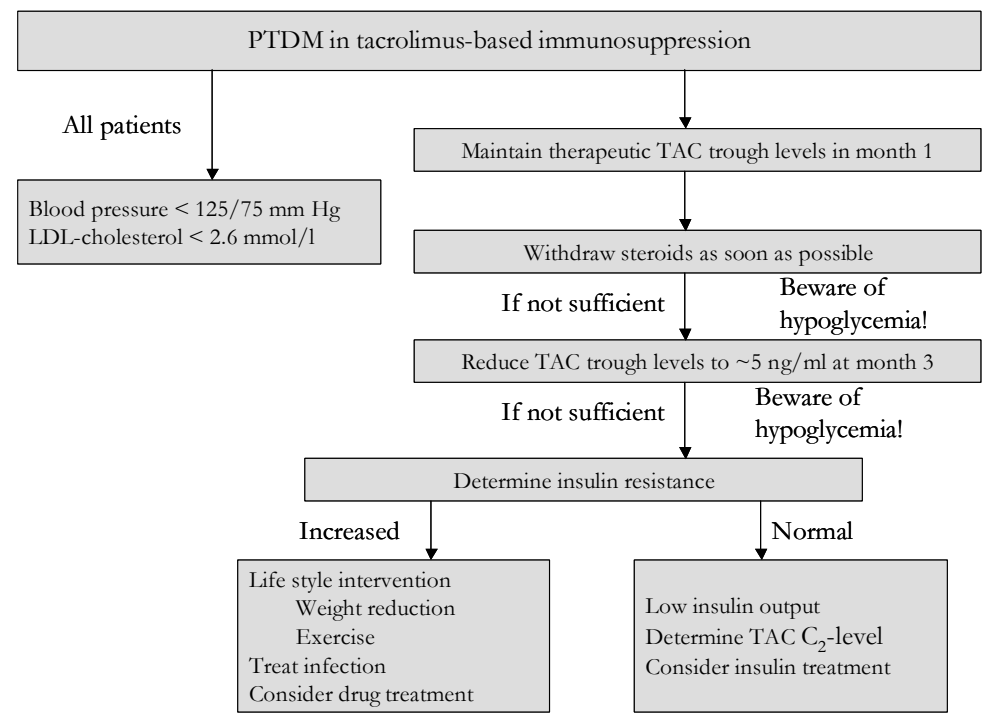

Figure 10.3 Flow chart of the management of post-transplant diabetes mellitus (PTDM) in TACbased immunosuppression for transplant recipients who have a low or intermediate immunological risk of acute rejection. In high-risk recipients, steroids may be reduced to $5 \mathrm{mg}$ maintenance. In the case of low insulin output, determination of TAC blood level two hours after dosing $\left(\mathrm{C}_{2}\right)$ may reveal high exposure to TAC in comparison to the trough level. Further reduction in the TAC dose will then be the best option.

\section{Cardiovascular risk profile}

Death with a functioning graft is a major cause of graft loss after successful renal transplantation. Cardiovascular disease is the major cause of death in renal transplant recipients ${ }^{141-143}$. Since prospective studies evaluating the effects of immunosuppressive drugs on firm cardiovascular endpoints like cardiovascular 
mortality, ischemic heart disease, or stroke are very scarce, surrogate endpoints, such as the known cardiovascular risk factors evolved from epidemiological studies, become important to determine the strategy. In Chapter 9, the effects of currently used immunosuppressive drugs on these risk factors are discussed.

In pivotal trials comparing TAC with CsA-Sandimmune ${ }^{1,2}$, evidence evolved that the side effects of both calcineurin blockers were substantially different for cardiovascular risk factors. Blood pressure seemed comparable, but the lipid profile was more favourable with the use of TAC. On the other hand, as discussed in the previous paragraph, PTDM occurred more in TAC-treated patients. Other studies with a cross-sectional design indicated that blood pressure might also be better controlled with TAC because TAC-treated patients needed significantly fewer anti-hypertensive drugs ${ }^{144,145}$.

In Chapter 2, we evaluated the cardiovascular side effects of TAC and CsA and observed that the TAC-treated patients indeed needed significantly fewer antihypertensive drugs to achieve similar blood pressure control. These patients had significantly lower cholesterol levels too ${ }^{10}$. The results have to be interpreted with caution, because fewer patients in the TAC group still used steroids. Steroids are well known to induce hypertension ${ }^{39,42,146,147}$ and hyperlipidemia ${ }^{148,149}$.

In recent years, it has been shown in healthy volunteers ${ }^{150}$ and renal transplant recipients ${ }^{144,151}$ that TAC, unlike CsA, does not induce vasoconstriction, hypertension, and an increase in serum creatinine. Ligtenberg et al. ${ }^{152}$ showed in a short-term cross-over study from CsA to TAC that blood pressure decreased with $10 \mathrm{~mm} \mathrm{Hg}$ during TAC therapy. Our results on hypertension were confirmed by the prospective study of Margreiter et al. comparing TAC to CsAmicroemulsion ${ }^{12}$. However, in the two-year follow-up publication of that study, the incidence of hypertension was reported to be comparable for both drugs ${ }^{32}$.

Hyperlipidemia occurred more often with CsA than with TAC. In a more recent report of the European trial, it was shown that cholesterol did not change after transplantation with TAC, whereas it increased after treatment with CsA ${ }^{153}$. The multicenter European trial comparing TAC with CsA-microemulsion confirmed our results with lower cholesterol in TAC-treated patients ${ }^{12,32}$.

Since transplant patients have better control of blood pressure and hyperlipidemia with TAC in the first years post-transplantation, it was questioned whether stable long-term CsA-treated patients would benefit from a conversion from CsA to TAC. Initial experience with conversion for hyperlipidemia indicated lower cholesterol levels and better control of blood pressure ${ }^{154}$.

In Chapter 7, the first randomized controlled trial on conversion from CsA to TAC is described ${ }^{137}$. Conversion was safe, no rejections were observed, and blood pressure decreased by about $5 \mathrm{mmHg}$. In Chapter 8 , the two-year results of that trial clearly showed that the results were sustained ${ }^{138}$. A European multicenter 
study evaluating the effects of conversion from CsA to TAC in patients with CsArelated side effects showed a similar improvement of $5 \mathrm{~mm} \mathrm{Hg}$ in diastolic blood pressure. In this study, the decrease in systolic blood pressure was as much as 15 $\mathrm{mmHg}^{155}$. Data correlating blood pressure and cardiovascular morbidity are lacking for transplant patients, but are well estimated for the general population. A mean decrease of just $3 \mathrm{mmHg}$ with ACE inhibitors already resulted in a decrease of $20 \%-30 \%$ of the cardiovascular risk, depending on the parameter studied: stroke, coronary heart disease, major cardiovascular events, or cardiovascular death ${ }^{156}$. If these data can be extrapolated to transplant patients, a reduction of that size by conversion from TAC to CsA would probably have a major impact on the burden of cardiovascular disease in renal transplant recipients. Hypertension also negatively influences graft surviva ${ }^{85-87,157}$ and contributes to chronic allograft nephropathy 158,159 . Recently, it was demonstrated that lowering blood pressure, even several years after transplantation, still improved both patient and graft survival ${ }^{160}$.

As shown in Chapter 7, conversion from CsA to TAC also lowered both total cholesterol and LDL-cholesterol (LDL-c). Triglycerides decreased as well ${ }^{137,161}$. Chapter 8 showed that these results were sustained during the two-year study period ${ }^{138}$. Total cholesterol decreased by about $0.5 \mathrm{mmol} / 1$ and LDL-c by $0.3 \mathrm{mmol} / \mathrm{l}$. These findings were in line with those of conversion studies due to hyperlipidemia $154,155,162$, although the decrease in cholesterol values in our study was somewhat lower. This may have been due to the selection of hyperlipidemic patients in the McCune et al. study and the use of statins in some of the patients in our study. Using statins reduces, but does not abolish, the impact of the conversion on lipid levels ${ }^{137}$.

In addition to lipid levels, the oxidation state of LDL-particles is an important factor in the development of atherosclerosis ${ }^{163,164}$. In Chapter 7, our study demonstrated that TAC not only lowered the levels of LDL-c, but also improved the oxidation state of LDL-c ${ }^{137}$. Before the results of our study were published, data on the oxidation of LDL were unequivocal. Some studies showed that TAC did not influence the oxidation of LDL ${ }^{165,166}$. Others did not observe a difference between TAC and $\mathrm{CsA}^{167}$ or even an enhanced susceptibility of LDL to oxidation with TAC compared to CsA-microemulsion ${ }^{168,169}$. The latter results might have been influenced by the fortification of CsA-microemulsion by $\alpha$-tocopherol, which has anti-oxidant properties. A Spanish conversion trial confirmed our results ${ }^{170}$.

Like hypertension, hyperlipidemia also contributes to the development of chronic allograft nephropathy and impairs graft survival $89,90,171$. Therefore, an improvement in the lipid profile after conversion from CsA to TAC may not only be beneficial for the cardiovascular risk, but it may also improve graft survival. To estimate the risk of cardiovascular disease with a combination of several risk factors, the Framingham risk score was developed from epidemiological research. 
This score includes gender, age, smoking, diabetes mellitus, hypertension, and both total and LDL-c, and it correlates well with the incidence of cardiovascular disease and mortality ${ }^{172}$. After conversion from CsA to TAC, a significant improvement in the Framingham risk score, up to two years after the intervention, was observed ${ }^{137,138}$. Thus, it can be concluded that TAC is more favourable with regard to the risk of developing cardiovascular disease. However, this risk score is not validated in renal transplant patients. Therefore, the results have to be considered with caution. Afterwards, a validation study in renal transplant recipients showed that non-traditional risk factors, such as $\mathrm{C}$-reactive protein and homocysteine, contributed significantly to the increased cardiovascular risk of these patients and that the Framingham risk score underestimated the real cardiovascular risk in high-risk transplant recipients ${ }^{173}$. It has also been shown that cardiovascular mortality is increased in patients with an increase in serum creatinine at one year after renal transplantation ${ }^{174}$. This factor has not yet been taken into account in risk scores.

Nevertheless, Waid recently reported a significantly lower incidence of cardiac conditions in patients with chronic renal allograft failure who were converted to TAC than in patients who stayed on $\mathrm{CsA}^{139}$. This report is actually the first to show that improvement in cardiovascular risk will decrease the burden of cardiovascular disease in renal transplant recipients.

\section{Renal function}

Renal function can be determined by measuring the clearance of a substance that is only filtrated in the glomeruli and neither re-absorbed nor secreted in the tubules, e.g., inulin or iothalamate. However, determining the GFR is impractical and expensive and, therefore, less applicable to daily clinical practice. Instead, renal function is usually expressed in terms of serum creatinine or creatinine clearance. Most serum creatinine is cleared by glomerular filtration only. However, a proportion is re-absorbed in the proximal tubule or secreted in the distal tubule. Therefore, creatinine clearance may underestimate or overestimate the real GFR ${ }^{175}$. Renal function is an important prognostic factor for long-term graft survival. Both renal function at one year post-transplantation and the slope of the decline (delta $(\Delta))$ in renal function clearly predict long-term graft survival11,176-178. Furthermore, decreased renal function is a strong risk factor for cardiovascular death after transplantation ${ }^{174}$. In addition, in transplantation of organs other than kidneys, impairment of renal function increases the risk of death by more than four times ${ }^{179}$. Therefore, preventing the loss of renal function is an important target in the treatment of renal transplant recipients.

Both TAC and CsA influence renal function. Initial reports showed similar histological changes in protocol biopsies ${ }^{180-182}$. In the main prospective trials, renal function at 6-12 months post-transplantation was comparable for TAC and both CsA-Sandimmune ${ }^{1,2}$ and CsA-microemulsion ${ }^{12}$. As we showed in our study in 
Chapter 2, renal function at one year post-transplantation was also comparable for both drugs ${ }^{10}$. However, data from the last five years provide more and more evidence that the nephrotoxicity of these two drugs differs.

Klein et al. showed that, in healthy volunteers during 14 days of administration, TAC did not influence renal hemodynamics, whereas CsA induced renal vasoconstriction and impaired the GFR (Figure 10.4) ${ }^{150}$. In renal transplant recipients, Radermacher et al. showed that renal vasoconstriction, expressed as the resistance index measured by Doppler ultrasound at the level of the interlobar artery, was significantly higher in the first two months post-transplantation in CsA-treated patients compared to TAC-treated patients ${ }^{144}$. Recently, Nankivell et al. described how CsA resulted in phasic renal hypoperfusion with a maximum effect about one hour after dosing, whereas TAC showed no effect ${ }^{151}$.

\section{Renal hemodynamics in healthy subjects}

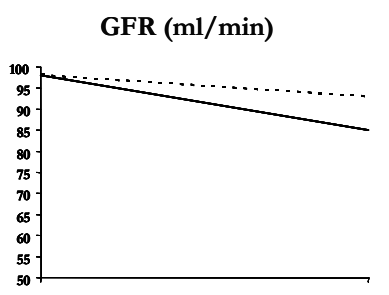

$\operatorname{ERPF}\left(\mathrm{ml} / \mathrm{min} / 1.73 \mathrm{~m}^{2}\right)$

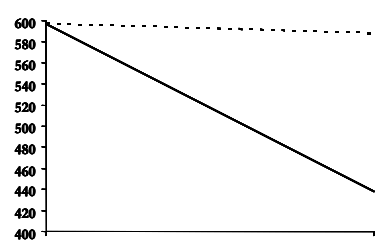

MAP (mmHg)

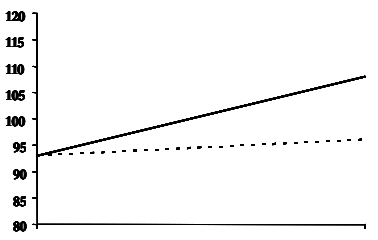

Tacrolimus
$\operatorname{RBF}\left(\mathrm{ml} / \mathrm{min} / 1.73 \mathrm{~m}^{2}\right)$

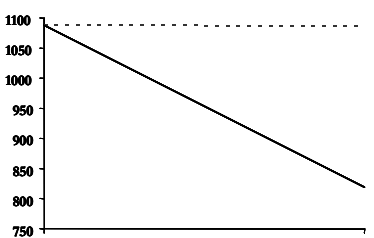

Creatinine $(\mu \mathrm{mol} / 1)$

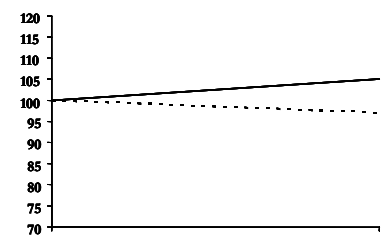

Cyclosporin

Figure 10.4 Differential effect of 14 days treatment with TAC or CsA on renal hemodynamics and GFR in healthy volunteers.

Figure based on: Klein et al. ${ }^{150}$.

Van Duijnhoven et al. analyzed uncomplicated transplantations from living donors and showed that the creatinine clearance achieved at one year posttransplantation in TAC-treated recipients was comparable to the remaining creatinine clearance of the donors ${ }^{183}$. Kaplan et al. compared paired donated kidneys, one of which was received by a recipient treated with TAC and the other by a recipient treated with CsA. At all time points, recipients treated with TAC had better renal function. However, in that study, the slope of the decline in renal 
function did not differ between the groups ${ }^{184}$. In contrast, a recent, CsA and TAC AUC-controlled single center study of paired kidneys showed a decline in renal function in CsA-treated patients compared to stable renal function in TAC-treated patients ${ }^{185}$. Also, in a recent publication of a randomized trial comparing TAC+MMF versus sirolimus (RAPA) +MMF there was no difference in GFR (measured by iothalamate) between TAC-based and calcineurin inhibitor (CNI)free arms up to two years post-transplantation ${ }^{186}$.

Interestingly, as we demonstrated in Chapter 7, conversion from CsA to TAC in stable renal transplant recipients with no clinical deterioration in renal function more than a median of five years after a successful transplantation resulted in an improvement in the patient's transplant function ${ }^{137}$. The two-year follow-up data of that trial presented in Chapter 8 still confirmed a preserved transplant function in the conversion group in contrast to a gradual decline in creatinine clearance in the group that stayed on $\mathrm{CsA}^{138}$. In addition, conversion, even after many years (median ten years) of CsA use, resulted in an improvement in renal function (Gelens et al, unpublished). Improved renal function after conversion was also reported in children with chronic rejection ${ }^{187}$ and in other conversion studies in adults with chronic transplant nephropathyy ${ }^{139,188-190}$ or CsA-related side effects $^{155,188}$.

Follow-up data from the US pivotal trial showed comparable five-year graft survival between the two groups in an intention-to-treat analysis ${ }^{36}$. However, a substantial proportion of the CsA-treated patients were converted to TAC, predominantly due to a lack of efficacy. After censoring for these converted patients, it was shown that patients treated with TAC had better graft survival and a significantly better creatinine clearance. The two-year results of the European multicenter study comparing TAC and CsA-microemulsion also showed a better creatinine clearance in the TAC-treated patients ${ }^{32}$. Jurewicz evaluated renal function by measuring GFR and observed a better GFR from three months posttransplantation onwards in the TAC-treated patients compared to the CsAmicroemulsion-treated patients. The difference in GFR between the groups increased up to five years post-transplantation ${ }^{11}$. Hunsicker et al. ${ }^{191}$ and Halloran ${ }^{192}$ advised assessing the slope of the loss of renal function as an important parameter for the evaluation of renal function and the success of a certain therapy. Jurewicz showed that the slope of the GFR in CsAmicroemulsion-treated patients significantly declined in contrast to TAC-treated patients ${ }^{11}$. Analysis of the United States Renal Data System confirmed this observation. The slope of the decline in renal function was horizontal in the TACtreated patients, whereas both CsA-microemulsion and CsA-Sandimmune-treated patients showed a gradual decline per year ${ }^{193}$.

Besides clinical observations of better renal function with TAC, there are indications that TAC is more beneficial for the kidney on a molecular and histology basis as well. However, the data are not unequivocal. In a rat ischemia- 
reperfusion model, TAC significantly reduced the upregulation of fibrosisassociated genes in contrast to $\mathrm{CsA}^{194}$. Isolated glomeruli incubated for 24 hours with either TAC or CsA showed increased expression of extracellular matrix turnover with $\mathrm{CsA}$ in contrast to $\mathrm{TAC}^{195}$. Isolated glomeruli from protocol biopsies expressed fewer fibrosis-associated genes, except for transforming growth factor (TGF)- $\beta_{1}$ in TAC-treated patients compared to CsAmicroemulsion-treated patients ${ }^{196}$. In renal biopsies, it was shown that CsA-treated patients expressed more active TGF- $\beta 1^{31,197}$. However, this observation was not confirmed in another study using protocol biopsies from stable renal transplant recipients ${ }^{198}$. Khanna et al. observed an even higher expression of TGF- $\beta_{1}$ in biopsies taken from patients with renal insufficiency due to TAC nephrotoxicity than in biopsies taken from patients with CsA nephrotoxicity ${ }^{199}$. Another study with protocol biopsies showed that TAC-treated patients had a significantly lower acute score, representing fewer acute rejections, and less transplant glomerulopathy, but a comparable chronic lesion score compared to CsAmicroemulsion-treated patients ${ }^{24}$. In contrast, Baboolal et al. showed that TACtreated patients not only expressed fewer pro-fibrotic growth factors than CsAmicroemulsion, but also developed significantly less chronic renal injury ${ }^{31}$. Their data are in accordance with the studies of Nankivell et al. and Murphy et al., which also showed a significant increase in interstitial fibrosis in patients treated with CsA compared to TAC200,201. Recently, however, AUC-guided TAC dosing was prospectively compared with AUC-guided CsA-microemulsion on fibrosis formation in the first year post-transplantation. In this study, no significant differences were observed in the expression of TGF- $\beta_{1}$ and other fibrosis parameters. Since TGF- $\beta_{1}$-formation is increased by acute rejection episodes, the former studies might have been biased by the higher incidence of acute rejection in CsA-treated patients ${ }^{26,202}$.

A summary of the evidence that TAC has low nephrotoxic properties is given in Table 10.3.

In conclusion, the better preserved renal function with TAC compared to CsA will be the result of direct improvements in renal hemodynamics, lower acute and subclinical rejection rates, possibly less induction of fibrosis formation, and less indirect renal toxicity by improvements in hypertension and hyperlipidemia. A comparison of secondary outcome parameters is presented in Figure 10.5. 
Table 10.3 Evidence for a low nephrotoxic potential of tacrolimus.

\begin{tabular}{|c|c|}
\hline Observation & Reference \\
\hline No effect on renal hemodynamics in healthy volunteers & Klein 150 \\
\hline $\begin{array}{l}\text { No transplant hypoperfusion shortly after dosing with TAC } \\
\text { compared to CsA }\end{array}$ & Nankivell 151 \\
\hline $\begin{array}{l}\text { Comparable creatinine clearance between recipient of } \\
\text { uncomplicated living donor and the donor }\end{array}$ & Duijnhoven ${ }^{183}$ \\
\hline Stable creatinine clearance after the first year post-transplantation & Jurewicz $^{11}$ \\
\hline Improvement in renal function by conversion from $\mathrm{CsA}$ to $\mathrm{TAC}$ & Artz 137,138 \\
\hline $\begin{array}{l}\text { Rescue of CsA-nephrotoxicity or chronic allograft nephropathy } \\
\text { by conversion to TAC }\end{array}$ & $\begin{array}{l}\text { Margreiter }{ }^{155} \text {, Cantarovitch }{ }^{188} \text {, } \\
\text { Hohage }^{189}, \text { Meier }^{190}\end{array}$ \\
\hline $\begin{array}{l}\text { Stable transplant function in paired kidneys by TAC compared to } \\
\text { decline with CsA }\end{array}$ & Cheung 185 \\
\hline $\begin{array}{l}\text { Same GFR with TAC+MMF versus sirolimus }+ \text { MMF at } 2 \text { years } \\
\text { post-transplantation }\end{array}$ & $\operatorname{Larson}^{186}$ \\
\hline
\end{tabular}

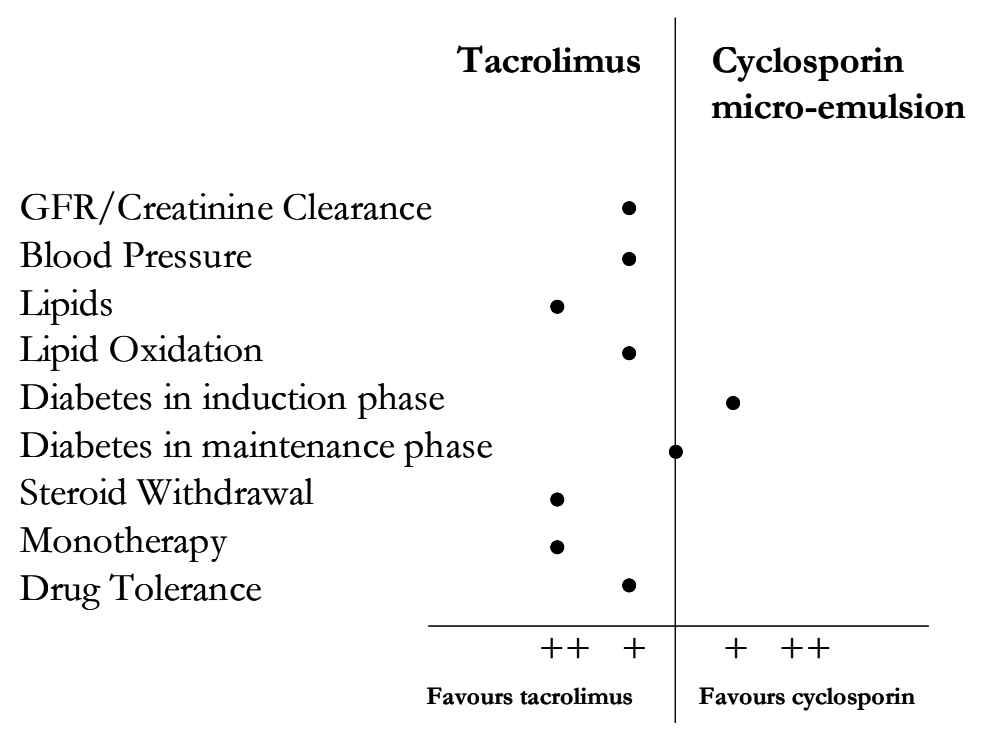

Figure 10.5 Comparison of tacrolimus with cyclosporin-microemulsion for secondary outcome parameters. GFR=glomerular filtration rate 


\section{Conclusion}

In this thesis, it has been shown that TAC is more potent in preventing acute rejections than not only CsA-Sandimmune but also CsA-microemulsion. Moreover, the cardiovascular risk profile with regard to hypertension and hyperlipidemia is more favourable, and new evidence supports a lower nephrotoxic potential. Due to its potency, steroid withdrawal is feasible, even early after transplantation, for up to $80 \%$ of the general transplant population, and TAC has the potential to treat about $70 \%$ of this population with monotherapy. In the near future, TAC will also be available in a slow release formulation, allowing once-daily dosing and improving compliance ${ }^{203,204}$.

Steroids can be withdrawn more rapidly, with a low risk of adrenal insufficiency, if performed within six months after the start of therapy. When withdrawing steroids, one must take into account that they induce the cytochrome system and enhance the metabolism of TAC. Therefore, cessation of steroids can lead to a significant increase in TAC trough levels, with a subsequent increase in serum creatinine. This can be misinterpreted as an episode of rejection.

With regard to the development of diabetes mellitus, TAC impairs insulin secretion and has no direct effect on insulin resistance, whereas steroids enhance insulin resistance and have no effect on insulin secretion. The risk of developing diabetes with TAC can be diminished by avoidance or early withdrawal of steroids and TAC trough level reduction.

Furthermore, not only do transplant patients who have been treated from transplantation onwards benefit from the advantages of TAC, but also stable patients who have been treated for years with CsA benefit from conversion to TAC. They have better preserved graft function and an improved cardiovascular risk profile as a result of lower blood pressure, lower lipid levels, less lipid oxidation, and lower fibrinogen levels.

Although studies may show the benefits of one therapy over another, patients still have to tolerate these drugs. Therefore, it is important that this thesis has shown that, compared to CsA, TAC is also better tolerated by patients. 


\section{References}

1. Pirsch JD, Miller J, Deierhoi MH, Vincenti F and Filo RS for the FK506 Kidney Transplant Study Group. A comparison of tacrolimus (FK506) and cyclosporine for immunosuppression after cadaveric renal transplantation. Transplantation 1997;63:977-83.

2. Mayer AD, Dmitrewski J, Squifflet J-P, Besse T, Grabensee B, Klein B, Eigler FW, Heemann U, Pichlmayr R, Behrend M, Vanrenterghem Y, Donck J, Hooff J van, Christiaans M, Morales JM, Andres A, Johnson RWG, Short C, Buchholz B, Rehmert N, Land W, Schleibner S, Forsythe JLR, Talbot D, Neumayer H-H, Hauser I, Ericzon B-G, Brattström C, Claesson K, Mühlbacher F and Pohanka E. Multicenter randomized trial comparing tacrolimus (FK 506) and cyclosporin in the prevention of renal allograft rejection. A report of the European Tacrolimus Multicenter Renal Study Group. Transplantation 1997;64:436-43.

3. Goto T, Kino T, Hatanaka H, Nishiyama M, Okuhara M, Kohsaka M, Aoki H and Imanaka H. Discovery of FK-506, a novel immunosuppressant isolated from Streptomyces tsukubaensis. Transplant Proc 1987;19:4-8.

4. Friman S and Bäckman L. A new microemulsion formulation of cyclosporin. Pharmacokinetic and clinical features. Clin Pharmacokinet 1996;30:181-93.

5. Niese D on behalf of the International Sandimmun Neoral Study Group. A double-blind randomized study of Sandimmun Neoral versus Sandimmun in new renal transplant recipients: results after twelve months. Transplant Proc 1995;27:1849-56.

6. Keown P and Niese D on behalf of the International Sandimmun Neoral Study Group. Cyclosporine microemulsion increases drug exposure and reduces acute rejection without incremental toxicity in de novo renal transplantation. Kidney Int 1998;54:938-44.

7. Pollard SG, Lear PA, Ready AR, Moore RH and Johnson RWG on behalf of the UK Neoral Renal Study Group. Comparison of microemulsion and conventional formulations of cyclosporin A in preventing acute rejection in de novo kidney transplant patients. Transplantation 1999;68:1325-31.

8. Morris-Stiff G, Ostrowski K, Balaji V, Moore R, Darby C, Lord R and Jurewicz WA. Prospective randomised study comparing tacrolimus (Prograf) and cyclosporine (Neoral) as primary immunosuppression in cadaveric renal transplants at a single institution: interim report of the first 80 cases. Transpl Int 1998;11(S1):S334-6.

9. Undre NA, Hooff J van, Christiaans M, Vanrenterghem Y, Donck J, Heeman U, Kohnle M, Zanker W, Morales JM, Andrés A, Schäfer A and Stevenson P. Low systemic exposure to tacrolimus correlates with acute rejection. Transplant Proc 1999;31:296-8.

10. Boots JMM, Duijnhoven EM, Christiaans MHL, Nieman FHM, Suylen RJ van and Hooff JP van. Single center experience with tacrolimus versus cyclosporin-Neoral in renal transplant recipients. Transpl Int 2001;14:370-83.

11. Jurewicz WA. Tacrolimus versus ciclosporin immunosuppression: long-term outcome in renal transplantation. Nephrol Dial Transplant 2003;18(S1):i7-11.

12. Margreiter $\mathrm{R}$ for the European Tacrolimus vs Ciclosporin Microemulsion Renal Transplantation Study Group. Efficacy and safety of tacrolimus compared with ciclosporin microemulsion in renal transplantation: a randomised multicentre study. Lancet 2002;359: 741-6. 
13. Webster AC, Woodroffe RC, Taylor RS, Chapman JR and Craig JC. Tacrolimus versus ciclosporin as primary immunosuppression for kidney transplant recipients: meta-analysis and meta-regression of randomised trial data. Br Med J 2005;331:810-21.

14. Saase JLCM, Woude FJ van der, Thorogood J, Hollander AAMJ, Es LA van, Weening JJ, Bockel JH van and Bruijn JA. The relation between acute vascular and interstitial renal allograft rejection and subsequent chronic rejection. Transplantation 1995;59:1280-5.

15. Meier-Kriesche H-U, Arndorfer JA and Kaplan B. Association of antibody induction with short- and long-term cause specific mortality in renal transplantation. J Am Soc Nephrol 2002; 13:769-72.

16. Woodle ES, Thistlethwaite JR, Gordon JH, Laskow D, Deierhoi MH, Burdick J, Pirsch JD, Sollinger H, Vincenti F, Burrows L, Schwartz B, Danovitch GM, Wilkinson AH, Shaffer D, Simpson MA, Freeman RB, Rohrer RJ, Mendez R, Aswad S, Munn SR, Wiesner RH, Delmonico FL, Neylan J and Whelchel J. A multicenter trial of FK506 (tacrolimus) therapy in refractory acute renal allograft rejection. A report of the Tacrolimus Kidney Transplantation Rescue Study Group. Transplantation 1996;62:594-9.

17. Woodle ES, Cronin D, Newell KA, Millis JM, Bruce DS, Piper JB, Haas M, Josephson MA and Thistlethwaite JR. Tacrolimus therapy for refractory acute renal allograft rejection. Definition of the histologic response by protocol biopsies. Transplantation 1996;62:906-10.

18. Jordan ML, Naraghi R, Shapiro R, Smith D, Vivas CA, Scantlebury VP, Gritsch HA, McCauley J, Randhawa P, Demetris AJ, McMichael J, Fung JJ and Starzl TE. Tacrolimus rescue therapy for renal allograft rejection - five-year experience. Transplantation 1997;63:223-8.

19. Nankivell BJ, Fenton-Lee CA, Kuypers DRJ, Cheung E, Allen RDM, O'Connell PJ and Chapman JR. Effect of histological damage on long-term kidney transplant outcome. Transplantation 2001;71:515-23.

20. Nankivell BJ, Borrows RJ, Fung CL, O'Connell PJ, Allen RD and Chapman JR. Natural history, risk factors, and impact of subclinical rejection in kidney transplantation. Transplantation 2004;78:242-9.

21. Nankivell BJ, Borrows RJ, Fung CL-S, O'Connell PJ, Allen RDM and Chapman JR. The natural history of chronic allograft nephropathy. N Engl J Med 2003;349:2326-33.

22. Miyagi M, Ishikawa Y, Mizuiri S, Aikawa A, Ohara T and Hasegawa A. Significance of subclinical rejection in early renal allograft biopsies for chronic allograft dysfunction. Clin Transplant 2005;19:456-65.

23. Gloor JM, Cohen AJ, Lager DJ, Grande JP, Fidler ME, Velosa JA, Larson TS, Schwab TR, Griffin MD, Prieto M, Nyberg SL, Sterioff S, Kremers WK and Stegall MD. Subclinical rejection in tacrolimus-treated renal transplant recipients. Transplantation 2002;73:1965-8.

24. Moreso F, Seron D, Carrera M, Gil-Vernet S, Cruzado JM, Hueso M, Fulladosa X, Ramos R, Ibernon M, Castelao AM and Grinyo JM. Baseline immunosuppression is associated with histological findings in early protocol biopsies. Transplantation 2004;78:1064-8.

25. Nashan B, Bock A, Bosmans J-L, Budde K, Fijter H de, Jaques B, Johnston A, Lück R, Midtvedt K, Pallardó LM, Ready A, Salamé E, Salizzoni M, Suarez F and Thervet E. Use of Neoral $C_{2}$ monitoring: a European consensus. Transpl Int 2005;18:768-78.

26. Rowshani AT, Scholten EM, Bemelman F, Eikmans M, Idu M, Groningen MCR van, Surachno JS, Mallat MJK, Paul LC, Fijter JW de, Bajema IM, Berge I ten and Florquin S. No difference in degree of interstitial sirius red-stained area in serial biopsies from area under concentration-over-time curves-guided cyclosporine versus tacrolimus-treated renal transplant recipients at one year. J Am Soc Nephrol 2006;17:305-12. 
27. Pirsch JD, Ploeg RJ, Gange S, D'Alessandro AM, Knechtle SJ, Sollinger HW, Kalayoglu M and Belzer FO. Determinants of graft survival after renal transplantation. Transplantation 1996;61: 1581-6.

28. Matas A. Chronic rejection in renal transplant recipients - risk factors and correlates. Clin Transplant 1994;8:332-5.

29. Lindholm A, Ohlman S, Albrechtsen D, Tufveson G, Persson H and Persson NH. The impact of acute rejection episodes on long-term graft function and outcome in 1347 primary renal transplants treated by 3 cyclosporine regimens. Transplantation 1993;56:307-15.

30. Ishikawa A, Flechner SM, Goldfarb DA, Myles JL, Modlin CS, Boparai N, Papajcik D, Mastroianni B and Novick AC. Quantitative assessment of the first acute rejection as a predictor of renal transplant outcome. Transplantation 1999;68:1318-24.

31. Baboolal K, Jones GA, Janezic A, Griffiths DR and Jurewicz WA. Molecular and structural consequences of early renal allograft injury. Kidney Int 2002;61:686-96.

32. Krämer BK, Montagnino G, Castillo D del, Margreiter R, Sperschneider H, Olbricht CJ, Krüger B, Ortuño J, Köhler H, Kunzendorf U, Stummvoll H-K, Tabernero JM, Mühlbacher F, Rivero M and Arias M for the European Tacrolimus vs Cyclosporin Microemulsion Renal Transplantation Study Group. Efficacy and safety of tacrolimus compared with cyclosporin A microemulsion in renal transplantation: 2 year follow-up results. Nephrol Dial Transplant 2005;20:968-73.

33. Danovitch GM. Immunosuppressive medications for renal transplantation: A multiple choice question. Kidney Int 2001;59:388-402.

34. Hunsicker LG and Bennett LE. Design of trials of methods to reduce late renal allograft loss: The price of success. Kidney Int 1995;52)S52):S120-3.

35. Gjertson DW, Cecka JM and Terasaki PI. The relative effects of FK506 and cyclosporine on short- and long-term kidney graft survival. Transplantation 1995;60:1384-8.

36. Vincenti F, Jensik SC, Filo RS, Miller J and Pirsch J. A long-term comparison of tacrolimus (FK506) and cyclosporine in kidney transplantation: evidence for improved allograft survival at five years. Transplantation 2002;73:775-82.

37. Mayer AD for the European Tacrolimus Multicentre Renal Study Group. Chronic rejection and graft half-life: Five year follow-up of the European Tacrolimus Multicenter Renal Study. Transplant Proc 2002;34:1491-2.

38. Sinclair NR for the Canadian Multicentre Transplant Study Group. Low-dose steroid therapy in cyclosporin-treated renal transplant recipients with well-functioning grafts. Can Med Assoc J 1992;147:645-57.

39. Hollander AAMJ, Hené RJ, Hermans J, Es LA van and Woude FJ van der. Late prednisone withdrawal in cyclosporine-treated kidney transplant patients: a randomized study. J Am Soc Nephrol 1997;8:294-301.

40. Ratcliffe PJ, Dudley CRK, Higgins RM, Firth JD, Smith B and Morris PJ. Randomised controlled trial of steroid withdrawal in renal transplant recipients receiving triple immunosuppression. Lancet 1996;348:643-8.

41. Schulak JA, Mayes JT, Moritz CE and Hricik DE. A prospective randomized trial of prednisone versus no prednisone maintenance therapy in cyclosporine-treated and azathioprine-treated renal transplant patients. Transplantation 1990;49:327-32. 
42. Ahsan N, Hricik D, Matas A, Rose S, Tomlanovich S, Wilkinson A, Ewell M, McIntosh M, Stablein D and Hodge E for the Steroid Withdrawal Group. Prednisone withdrawal in kidney transplant recipients on cyclosporin and mycophenolate mofetil - a prospective randomized study. Transplantation 1999;68:1865-74.

43. Kasiske BL, Chakkera HA, Louis TA and Ma JZ. A meta-analysis of immunosuppression withdrawal trials in renal transplantation. J Am Soc Nephrol 2000;11:1910-7.

44. Shapiro R, Jordan M, Scantlebury V, Fung J, Jensen C, Tzakis A, McCauley J, Carroll P, Ricordi C, Demetris AJ, Mitchell S, Jain A, Iwaki Y, Kobayashi M, Reyes J, Todo S, Hakala TR, Simmons RL and Starzl TE. FK 506 in clinical kidney transplantation. Transplant Proc 1991;23:3065-7.

45. Shapiro R, Jordan ML, Scantlebury VP, Vivas C, Fung JJ, McCauley J, Randhawa P, Demetris AJ, Irish W, Mitchell S, Hakala TR, Simmons RL and Starzl TE. A prospective randomized trial of FK506-based immunosuppression after renal transplantation. Transplantation 1995;59: 485-90.

46. Wood JB, Frankland AW, James VHT and Landon J. A rapid test of adrenocortical function. Lancet 1965;1:243-5.

47. LaRochelle GE jr, LaRochelle AG, Ratner RE and Borenstein DG. Recovery of the hypothalamic-pituitary-adrenal (HPA) axis in patients with rheumatic diseases receiving lowdose prednisone. Am J Med 1993;95:258-64.

48. Kane KF, Emery P, Sheppard MC and Stewart PM. Assessing the hypothalamo-pituitaryadrenal axis in patients on long-term glucocorticoid therapy: the short synacthen versus the insulin tolerance test. Q J Med 1995;88:263-7.

49. Miozzari $\mathrm{M}$ and Ambühl PM. Steroid withdrawal after long-term medication for immunosuppressive therapy in renal transplant patients: adrenal response and clinical implications. Nephrol Dial Transplant 2004;19:2615-21.

50. Sattler M, Guengerich FP, Yun CH, Christians U and Sewing KF. Cytochrome P-450 3A enzymes are responsible for biotransformation of FK506 and rapamycin in man and rat. Drug Metab Dispos 1992;20:753-61.

51. Shiraga T, Matsuda H, Nagase K, Iwasaki K, Noda K, Yamazaki H, Shimada T and Funae Y. Metabolism of FK506, a potent immunosuppressive agent, by cytochrome P450 3A enzymes in rat, dog and human liver microsomes. Biochem Pharmacol 1994;47:727-35.

52. Shimada T, Terada A, Yokogawa K, Kaneko H, Nomura M, Kaji K, Kaneko S, Kobayashi K and Miyamoto K. Lowered blood concentration of tacrolimus and its recovery with changes in expression of CYP3A and P-glycoprotein after high-dose steroid therapy. Transplantation 2002;74:1419-24.

53. Watkins PB, Murray SA, Winkelman LG, Heuman DM, Wrighton SA and Guzelian PS. Erythromycin breath test as an assay of glucocorticoid-inducible liver cytochromes P-450. Studies in rats and patients. J Clin Invest 1989;83:688-97.

54. Villikka K, Varis T, Backman JT, Neuvonen PJ and Kivistö KT. Effect of methylprednisolone on CYP3A4-mediated drug metabolism in vivo. Eur J Clin Pharmacol 2001;57:457-60.

55. McCune JS, Hawke RL, LeCluyse EL, Gillenwater HH, Hamilton G, Ritchie J and Lindley C. In vivo and in vitro induction of human cytochrome P4503A4 by dexamethasone. Clin Pharmacol Ther 2000;68:356-66. 
56. Hesselink DA, Ngyuen H, Wabbijn M, Smak Gregoor PJ, Steyerberg EW, Riemsdijk IC van, Weimar $\mathrm{W}$ and Gelder $\mathrm{T}$ van. Tacrolimus dose requirement in renal transplant recipients is significantly higher when used in combination with corticosteroids. Br J Clin Pharmacol 2003; 56:327-30.

57. Duijnhoven EM van, Boots JMM, Christiaans MHL, Stolk LML, Undre NA and Hooff JP van. Increase in tacrolimus trough levels after steroid withdrawal. Transpl Int 2003;16:721-5.

58. Grewal HP, Thistlethwaite JR, Loss GE, Bruce DS, Siegel CT, Cronin DC, Newell KA, Millis JM and Woodle ES. Corticosteroid cessation 1 week following renal transplantation using tacrolimus/mycophenolate mofetil based immunosuppression. Transplant Proc 1998;30: 1378-9.

59. Boots JMM, Christiaans MHL, Duijnhoven EM van, Suylen R-J van and Hooff JP van. Early steroid withdrawal in renal transplantation with tacrolimus dual therapy: A pilot study. Transplantation 2002;74:1703-9.

60. Calconi $G$ and Vianello A for the Italian and Spanish Tacrolimus Study Group. One-year follow-up of a large European trial comparing dual versus triple tacrolimus-based immunosuppressive regimens following renal transplantation. Transplant Proc 2001;33:1021-4.

61. Squifflet J-P, Bäckman L, Claesson K, Dietl KH, Ekberg H, Forsythe JLR, Kunzendorf U, Heemann U, Land W, Morales JM, Mühlbacher F, Talbot D, Taube D, Tyden G, Hooff JP van, Schleibner S and Vanrenterghem Y for the European Tacrolimus-MMF Renal Study Group. Dose optimization of mycophenolate mofetil when administered with a low dose of tacrolimus in cadaveric renal transplant recipients. Transplantation 2001;72:63-9.

62. Chang RWS, Snowden S, Palmer A, Kwan JTC, Nicholson M, Kashi SH, Fernando ON, Perner F and Neild GH. European randomised trial of dual versus triple tacrolimus-based regimens for control of acute rejection in renal allograft recipients. Transpl Int 2001;14:384-90.

63. Coupes BM, Speake M, Johnson RW, Roberts IS, Campbell B and Short CD. Tacrolimus monotherapy in renal transplantation: four-year data. Transplant Proc 2002;34:1612-3.

64. Boots JMM, Christiaans MHL, Undre NA and Hooff JP van. Pretransplant pharmacokinetics: does it predict the dose of tacrolimus after renal transplantation? Transplant Proc 2002;34:3171-2.

65. Buijsch RAM op den, Christiaans MHL, Stolk LML, Vries JE de, Cheung CY, Undre NA, Hooff JP van, Dieijen-Visser MP van and Bekers O. Tacrolimus pharmacokinetics and pharmacogenetics: influence of multidrug resistance gene-1 (MDR1) and cytochrome (CYP) 3A polymorphisms. Submitted.

66. Matas AJ, Ramcharan T, Paraskevas S, Gillingham KJ, Dunn DL, Gruessner RWG, Humar A, Kandaswamy R, Najarian JS, Payne WD and Sutherland DER. Rapid discontinuation of steroids in living donor kidney transplantation: A pilot study. Am J Transplant 2001;1:278-83.

67. Meulen CG ter, Riemsdijk I van, Hené RJ, Christiaans MHL, Borm GF, Gelder T van, Hilbrands LB, Weimar W and Hoitsma AJ. Steroid-withdrawal at 3 days after renal transplantation with anti-IL-2 receptor alpha therapy: a prospective, randomized, multicenter study. Am J Transplant 2004;4:803-10.

68. Cole E, Landsberg D, Russell D, Zaltzman J, Kiberd B, Caravaggio C, Vasquez AR and Halloran P. A pilot study of steroid-free immunosuppression in the prevention of acute rejection in renal allograft recipients. Transplantation 2001;72:845-50. 
69. Rostaing L, Cantarovich D, Mourad G, Budde K, Rigotti P, Mariat C, Margreiter R, Capdevilla L, Lang P, Vialtel P, Ortuno-Mirete J, Charpentier B, Legendre C, Sanchez-Plumed J, Oppenheimer $\mathrm{F}$ and Kessler $\mathrm{M}$ for the CARMEN study group. Corticosteroid-free immunosuppression with tacrolimus, mycophenolate mofetil, and daclizumab induction in renal transplantation. Transplantation 2005;79:807-14.

70. Vanrenterghem Y, Hooff JP van, Squifflet JP, Salmela K, Rigotti P, Jindal RM, Pascual J, Ekberg H, Sicilia LS, Boletis JN, Grinyo JM and Rodriguez MA for the European Tacrolimus/MMF Renal Transplantation Study Group. Minimization of immunosuppressive therapy after renal transplantation: results of a randomized controlled trial. Am J Transplant 2005;5:87-95.

71. Hooff JP van, Squifflet JP, Wlodarczyk Z, Vanrenterghem Y and Paczek L. A prospective randomized multicenter study of tacrolimus in combination with sirolimus in renal-transplant recipients. Transplantation 2003;75:1934-9.

72. Isaacs RB, Nock SL, Spencer CE, Connors AF jr, Wang X-Q, Sawyer R and Lobo PI. Racial disparities in renal transplant outcomes. Am J Kidney Dis 1999;34:706-12.

73. Neylan JF for the US Renal Transplant Mycophenolate Mofetil Study Group. Immunosuppressive therapy in high-risk transplant patients. Dose-dependent efficacy of mycophenolate mofetil in African-American renal allograft recipients. Transplantation 1997;64:1277-82.

74. Roodnat JI, Zietse R, Mulder PGH, Rischen-Vos J, Gelder T van, IJzermans JNM and Weimar W. The vanishing importance of age in renal transplantation. Transplantation 1999;67:576-80.

75. Humar A, Hassoun A, Kandaswamy R, Payne WD, Sutherland DER and Matas AJ. Immunologic factors: the major risk for decreased long-term renal allograft survival. Transplantation 1999;68:1842-6.

76. Chapman JR, Taylor CJ, Ting A and Morris PJ. Immunoglobulin class and specificity of antibodies causing positive $\mathrm{T}$ cell crossmatches. Relationship to renal transplant outcome. Transplantation 1986;42:608-13.

77. Taylor CJ, Chapman JR, Ting A and Morris PJ. Characterization of lymphocytotoxic antibodies causing a positive crossmatch in renal transplantation. Transplantation 1989;48: 953-8.

78. Sanfilippo F, Vaughn WK, LeFor WM and Spees EK. Multivariate analysis of risk factors in cadaver donor kidney transplantation. Transplantation 1986;42:28-34.

79. Richards E, Schleibner S, Talbot D and the European Tacrolimus Multicentre Renal Study Group. An exploratory analysis of prognostic factors for patient outcome during the first year following renal transplantation. Transplant Proc 1998;30:1386-8.

80. Hooff JP van, Salmela K, Budde K, Pascual J, Rigotti P and Lang P for the Tacrolimus Steroid-Withdrawal Study Group. Long-term efficacy and safety of steroid-withdrawal in tacrolimus-treated patients: Results of a long-term follow-up study in renal transplant recipients. 2003;ESOT Venice Abstract 99.

81. Matas AJ, Kandaswamy R, Gillingham KJ, McHugh L, Ibrahim H, Kasiske B and Humar A. Prednisone-free maintenance immunosuppression - A 5-year experience. Am J Transplant 2005;5:2473-8.

82. Vanrenterghem Y, Lebranchu Y, Hené R, Oppenheimer F and Ekberg H for the Steroid Dosing Study Group. Double-blind comparison of two corticosteroid regimens plus mycophenolate mofetil and cyclosporin for prevention of acute renal allograft rejection. Transplantation 2000;70:1352-9. 
83. Veenstra DL, Best JH, Hornberger J, Sullivan SD and Hricik DE. Incidence and long-term cost of steroid-related side effects after renal transplantation. Am J Kidney Dis 1999;33:829-39.

84. Hricik DE, Bartucci MR, Moir EJ, Mayes JT and Schulak JA. Effects of steroid withdrawal on posttransplant diabetes mellitus in cyclosporine-treated renal transplant recipients. Transplantation 1991;51:374-7.

85. Opelz G, Wujciak T and Ritz E for the Collaborative Transplant Study. Association of chronic kidney graft failure with recipient blood pressure. Kidney Int 1998;53:217-22.

86. Mange KC, Cizman B, Joffe M and Feldman HI. Arterial hypertension and renal allograft survival. J Am Med Assoc 2000;283:633-8.

87. Mange KC, Feldman HI, Joffe MM, Fa K and Bloom RD. Blood pressure and the survival of renal allografts from living donors. J Am Soc Nephrol 2004;15:187-93.

88. Peschke B, Scheuermann, Geiger H, Bölscher S, Kachel H-G and Lenz T. Hypertension is associated with hyperlipidemia, coronary heart disease and chronic graft failure in kidney transplant recipients. Clin Nephrol 1999;51:290-5.

89. Dimény E, Wahlberg J, Lithell $\mathrm{H}$ and Fellström B. Hyperlipidaemia in renal transplantation risk factor for long-term graft outcome. Eur J Clin Invest 1995;25:574-83.

90. Roodnat JI, Mulder PGH, Zietse R, Rischen-Vos J, Riemsdijk IC van, IJzermans JNM and Weimar W. Cholesterol as an independent predictor of outcome after renal transplantation. Transplantation 2000;69:1704-10.

91. Morris PJ, Johnson RJ, Fuggle SV, Belger MA and Briggs JD on behalf of the HLA Task Force of the Kidney Advisory Group of the United Kingdom Transplant Support Service Authority (UKTSSA). Analysis of factors that affect outcome of primary cadaveric renal transplantation in the UK. Lancet 1999;354:1147-52.

92. Miles AMV, Sumrani N, Horowitz R, Homel P, Maursky V, Markell MS, Distant DA, Hong JH, Sommer BG and Friedman EA. Diabetes mellitus after renal transplantation. As deleterious as non-transplant-associated diabetes? Transplantation 1998;65:380-4.

93. Kasiske BL, Snyder JJ, Gilbertson D and Matas AJ. Diabetes mellitus after kidney transplantation in the United States. Am J Transplant 2003;3:178-85.

94. Bertoni E, Zanazzi M, Maria L di, Moscarelli L, Piperno R, Conti P, Dedola G, Bandini S, Tosi $\mathrm{P}$ and Salvadori M. Long-term steroid side effects in renal transplantation need a safe steroid withdrawal: a single-center experience. Transplant Proc 1998;30:1303-4.

95. Tarantino A, Montagnino G and Ponticelli C. Corticosteroids in kidney transplant recipients. Safety issues and timing of discontinuation. Drug Saf 1995;13:145-56.

96. Heisel O, Heisel R, Balshaw R and Keown P. New onset diabetes mellitus in patients receiving calcineurin inhibitors: a systematic review and meta-analysis. Am J Transplant 2004;4:583-95.

97. Pagano G, Bruno A, Cavallo-Perin P, Cesco L and Imbimbo B. Glucose intolerance after short-term administration of corticosteroids in healthy subjects. Prednisone, deflazacort, and betamethasone. Arch Intern Med 1989;149:1098-101.

98. Hooff JP van, Christiaans MHL and Duijnhoven EM van. Evaluating mechanisms of posttransplant diabetes mellitus. Nephrol Dial Transplant 2004;19(S6):vi8-12.

99. Sato T, Inagaki A, Uchida K, Ueki T, Goto N, Matsuoka S, Katayama A, Haba T, Tominaga Y, Okajima Y, Ohta K, Suga H, Taguchi S, Kakiya S, Itatsu T, Kobayashi T and Nakao A. Diabetes mellitus after transplant: relationship to pretransplant glucose metabolism and tacrolimus or cyclosporine A-based therapy. Transplantation 2003;76:1320-6. 
100. Duijnhoven EM van, Boots JMM, Christiaans MHL, Wolffenbuttel BHR and Hooff JP van. Influence of tacrolimus on glucose metabolism before and after renal transplantation: a prospective study. J Am Soc Nephrol 2001;12:583-8.

101. Nam JH, Mun JI, Kim SI, Kang SW, Choi KH, Park K, Ahn CW, Cha BS, Song YD, Lim SK, Kim KR, Lee HC and Huh KB. ß-cell dysfunction rather than insulin resistance is the main contributing factor for the development of postrenal transplantation diabetes mellitus. Transplantation 2001;71:1417-23.

102. Weir MR and Fink JC. Risk for posttransplant diabetes mellitus with current immunosuppressive medications. Am J Kidney Dis 1999;34:1-13.

103. Boots JMM, Duijnhoven EM van, Christiaans MHL, Wolffenbuttel BHR and Hooff JP van. Glucose metabolism in renal transplant recipients on tacrolimus: the effect of steroid withdrawal and tacrolimus trough level reduction. J Am Soc Nephrol 2002;13:221-7.

104. Fernandez LA, Lehmann R, Luzi L, Battezati A, Angelico MC, Ricordi C, Tzakis A and Alejandro R. The effects of maintenance doses of FK506 versus cyclosporin A on glucose and lipid metabolism after orthotopic liver transplantation. Transplantation 1999;68:1532-41.

105. Strumph P, Kirsch D, Gooding W and Carroll P. The effect of FK506 on glycemic response as assessed by the hyperglycemic clamp technique. Transplantation 1995;60:147-51.

106. Filler G, Neuschultz I, Vollmer I, Amendt P and Hocher B. Tacrolimus reversibly reduces insulin secretion in paediatric renal transplant recipients. Nephrol Dial Transplant 2000;15: $867-71$.

107. Redmon JB, Olson LK, Armstrong MB, Greene MJ and Robertson RP. Effects of tacrolimus (FK506) on human insulin gene expression, insulin mRNA levels, and insulin secretion in HIT-T15 cells. J Clin Invest 1996;98:2786-93.

108. Tamura K, Fujimura T, Tsutsumi T, Nakamura K, Ogawa T, Atumaru C, Hirano Y, Ohara K, Ohtsuka K and Shimomura K. Transcriptional inhibition of insulin by FK506 and positive involvement of FK506 binding protein-12 in pancreatic beta-cell. Transplantation 1995;59:1606-13.

109. Roth D, Milgrom M, Esquenazi V, Fuller L, Burke G and Miller J. Posttransplant hyperglycemia. Increased incidence in cyclosporine-treated renal allograft recipients. Transplantation 1989;47:278-81.

110. Yamamoto H, Akazawa S, Yamaguchi Y, Yokota A, Yamasaki H, Nakanishi T, Tahara D, Matsuya F, Saito Y and Nagataki S. Effects of cyclosporin A and low dosages of steroid on posttransplantation diabetes in kidney transplant recipients. Diabetes Care 1991;14:867-70.

111. Harris KP, Russell GI, Parvin SD, Veitch PS and Walls J. Alterations in lipid and carbohydrate metabolism attributable to cyclosporin A in renal transplant recipients. Br Med J 1986;292: 16-9.

112. Robertson RP. Cyclosporin-induced inhibition of insulin secretion in isolated rat islets and HIT cells. Diabetes 1986;35:1016-9.

113. Gillison SL, Bartlett ST and Curry DL. Inhibition by cyclosporine of insulin secretion--a beta cell-specific alteration of islet tissue function. Transplantation 1991;52:890-5.

114. First MR, Gerber DA, Hariharan S, Kaufman DB and Shapiro R. Posttransplant diabetes mellitus in kidney allograft recipients: incidence, risk factors, and management. Transplantation 2002;73:379-86.

115. Duijnhoven EM van, Christiaans MHL, Boots JMM, Nieman FHR, Wolffenbuttel BHR and Hooff JP van. Glucose metabolism in the first 3 years after renal transplantation in patients on tacrolimus versus cyclosporine-based immunosuppression. J Am Soc Nephrol 2002;13:213-20. 
116. Hjelmesaeth J, Hartman A, Kofstad J, Stenstrom J, Leivestad T, Egeland T and Fauchald P. Glucose intolerance after renal transplantation depends upon prednisolone dose and recipient age. Transplantation 1997;64:979-83.

117. Kumar MSA, Heifets M, Moritz MJ, Saeed MI, Khan SM, Fyfe B, Sustento-Riodeca N, Daniel JN and Kumar A. Safety and efficacy of steroid withdrawal two days after kidney transplantation: Analysis of results at three years. Transplantation 2006;81:832-9.

118. Mathew JT, Madhumathi R, Job V, Ratnaswamy S and Jacob CK. Post-transplant hyperglycaemia: a study of risk factors. Nephrol Dial Transplant 2003;18:164-71.

119. Tuomilehto J, Lindstrom J, Eriksson JG, Valle TT, Hamalainen H, Ilanne-Parikka P, Keinanen-Kiukaanniemi S, Laakso M, Louheranta A, Rastas M, Salminen V, Uusitupa M and for the Finnish Diabetes Prevention Study Group. Prevention of type 2 diabetes mellitus by changes in lifestyle among subjects with impaired glucose tolerance. $\mathrm{N}$ Engl J Med 2001;344:1343-50.

120. Knowler WC, Barrett-Connor E, Fowler SE, Hamman RF, Lachin JM, Walker EA and Nathan DM for the Diabetes Prevention Program Research Group. Reduction in the incidence of type 2 diabetes with lifestyle intervention or metformin. N Engl J Med 2002;346:393-403.

121. Grundy SM, Brewer HB, Cleeman JI, Smith SC, Lenfant C and for the conference participants. Definition of metabolic syndrome: report of the National Heart, Lung, and Blood Institute/American Heart Association conference on scientific issues related to definition. Circulation 2004;109:433-8.

122. Vries AP de, Bakker SJ, Son WJ van, Homan van der Heide JJ, The TH, Jong PE de and Gans RO. Insulin resistance as putative cause of chronic renal transplant dysfunction. Am J Kidney Dis 2003;41:859-67.

123. Vries AP de, Bakker SJ, Son WJ van, Homan van der Heide JJ, Ploeg RJ, The TH, Jong PE de and Gans RO. Metabolic syndrome is associated with impaired long-term renal allograft function;not all component criteria contribute equally. Am J Transplant 2004;4:1675-83.

124. Gaede P, Vedel P, Larsen N, Jensen GVH, Parving H-H and Pedersen O. Multifactorial intervention and cardiovascular disease in patients with type 2 diabetes. $\mathrm{N}$ Engl J Med 2003;348:383-93.

125. American Diabetes Association. Standards of medical care for patients with diabetes mellitus. Diabetes Care 2003;26(S1):S33-50.

126. Solomon CG. Reducing cardiovascular risk in type 2 diabetes. N Engl J Med 2003;348:457-9.

127. UK Prospective Diabetes Study (UKPDS) Group. Intensive blood-glucose control with sulphonylureas or insulin compared with conventional treatment and risk of complications in patients with type 2 diabetes (UKPDS 33). Lancet 1998;352:837-53.

128. Stratton IM, Adler AI, Neil AW, Matthews DR, Manley SE, Cull CA, Hadden D, Turner RC and Holman RR. Association of glycaemia with macrovascular and microvascular complications of type 2 diabetes (UKPDS 35): prospective observational study. Br Med J 2000;321:405-12.

129. Hooff JP van, Christiaans MHL and Duijnhoven EM van. Tacrolimus and posttransplant diabetes mellitus in renal transplantation. Transplantation 2005;79:1465-9.

130. Hooff JP van, Boots JMM, Duijnhoven EM van and Christiaans MHL. Dosing and management guidelines for tacrolimus in renal transplant patients. Transplant Proc 1999;31(S7A):54S-7. 
131. Davidson J, Wilkinson A, Dantal J, Dotta F, Haller H, Hernandez D, Kasiske BL, Kiberd B, Krentz A, Legendre C, Marchetti P, Markell M, van der Woude FJ and Wheeler DC for the International Expert Panel. New-onset diabetes after transplantation: 2003 International consensus guidelines. Proceedings of an international expert panel meeting. Barcelona, Spain, 19 February 2003. Transplantation 2003;75(S10):SS3-24.

132. Luther $\mathrm{P}$ and Baldwin D Jr. Pioglitazone in the management of diabetes mellitus after transplantation. Am J Transplant 2004;4:2135-8.

133. Baldwin D Jr and Duffin KE. Rosiglitazone treatment of diabetes mellitus after solid organ transplantation. Transplantation 2004;77:1009-14.

134. Dormandy JA, Charbonnel B, Eckland DJA, Erdmann E, Massi-Benedetti M, Moules IK, Skene AM, Tan MH, Lefèbvre PJ, Murray GD, Standl E, Wilcox RG, Wilhelmsen L, Betteridge J, Birkeland K, Golay A, Heine RJ, Korányi L, Laakso M, Mokán M, Norkus A, Pirags V, Podar T, Scheen A, Scherbaum W, Schernthaner G, Schmitz O, Skrha J, Smith U and Taton $\mathrm{J}$ on behalf of the PROactive investigators. Secondary prevention of macrovascular events in patients with type 2 diabetes in the PROactive Study (PROspective pioglitAzone Clinical Trial In macroVascular Events): a randomised controlled trial. Lancet 2005;366: 1279-89.

135. Villanueva $G$ and Baldwin D. Rosiglitazone therapy of posttransplant diabetes mellitus. Transplantation 2005;80:1402-5.

136. Scholten EM, Cremers SCLM, Schoemaker RC, Rowshani AT, Kan EJ van, Hartigh J den, Paul LC and Fijter JW de. AUC-guided dosing of tacrolimus prevents progressive systemic overexposure in renal transplant recipients. Kidney Int 2005;67:2440-7.

137. Artz MA, Boots JMM, Ligtenberg G, Roodnat JI, Christiaans MHL, Vos PF, Blom HJ, Sweep FCGJ, Demacker PNM and Hilbrands LB. Improved cardiovascular risk profile and renal function in renal transplant patients after randomized conversion from cyclosporine to tacrolimus. J Am Soc Nephrol 2003;14:1880-8.

138. Artz MA, Boots JMM, Ligtenberg G, Roodnat JI, Christiaans MHL, Vos PF, Moons P, Borm $\mathrm{G}$ and Hilbrands LB. Conversion from cyclosporine to tacrolimus improves quality-of-life indices, renal graft function, and cardiovascular risk profile. Am J Transplant 2004;4:937-45.

139. Waid T for the CRAF Study Group. Tacrolimus as secondary intervention vs. cyclosporine continuation in patients at risk for chronic renal allograft failure. Clin Transplant 2005;19: $573-80$.

140. Gelens M, Christiaans M and Hooff J van. Will PTDM develop after conversion from ciclosporin to tacrolimus in stable renal recipients. ATC Seattle 2005; Abstract 868.

141. Howard RJ, Patton PR, Reed AI, Hemming AW, Werf WJ van der, Pfaff WW, Srinivas TR and Scornik JC. The changing causes of graft loss and death after kidney transplantation. Transplantation 2002;73:1923-8.

142. Ojo AO, Hanson JA, Wolfe RA, Leichtman AB, Agodoa LY and Port FK. Long-term survival in renal transplant recipients with graft function. Kidney Int 2000;57:307-13.

143. Aakhus S, Dahl K and Widerøe TE. Cardiovascular disease in stable renal transplant patients in Norway: morbidity and mortality during a 5-yr follow-up. Clin Transplant 2004;18:596-604.

144. Radermacher J, Meiners M, Bramlage C, Kliem V, Behrend M, Schlitt HJ, Pichlmayr R, Koch $\mathrm{KM}$ and Brunkhorst R. Pronounced renal vasoconstriction and systemic hypertension in renal transplant recipients treated with cyclosporin A versus FK 506. Transpl Int 1998;11:3-10. 
145. Hohage H, Brückner D, Arlt M, Buchholz B, Zidek W and Spieker C. Influence of cyclosporin $\mathrm{A}$ and FK506 on $24 \mathrm{~h}$ blood pressure monitoring in kidney transplant recipients. Clin Nephrol 1996;45:342-4.

146. Kupin W, Venkat KK, Oh HK and Dienst S. Complete replacement of methylprednisolone by azathioprine in cyclosporine-treated primary cadaveric renal transplant recipients. Transplantation 1988;45:53-5.

147. Hariharan S, Schroeder TJ, Weiskittel P, Alexander JW and First MR. Prednisone withdrawal in HLA identical and one haplotype-matched live-related donor and cadaver renal transplant recipients. Kidney Int 1993;44(S43):S30-5.

148. Hricik DE and Schulak JA. Metabolic effects of steroid withdrawal in adult renal transplant recipients. Kidney Int 1993;44(S43):S26-9.

149. Sévaux RGL de, Hilbrands LB, Tiggeler RGWL, Koene RAP and Hoitsma AJ. A randomised, prospective study on the conversion from cyclosporine-prednisone to cyclosporineazathioprine at 6 months after renal transplantation. Transpl Int 1998;11(S1):S322-4.

150. Klein IH, Abrahams A, Ede T van, Hené RJ, Koomans HA and Ligtenberg G. Different effects of tacrolimus and cyclosporine on renal hemodynamics and blood pressure in healthy subjects. Transplantation 2002;73:732-6.

151. Nankivell BJ, Chapman JR, Bonovas G and Gruenewald SM. Oral cyclosporine but not tacrolimus reduces renal transplant blood flow. Transplantation 2004;77:1457-9.

152. Ligtenberg G, Hené RJ, Blankestijn PJ and Koomans HA. Cardiovascular risk factors in renal transplant patients: Cyclosporin A versus tacrolimus. J Am Soc Nephrol 2001;12:368-73.

153. Claesson K, Mayer AD, Squifflet J-P, Grabensee B, Eigler FW, Behrend M, Vanrenterghem Y, Hooff J van, Morales JM, Johnson RWG, Buchholz B, Land W, Forsythe JLR, Neumayer H$H$, Ericzon B-G and Mühlbacher F. Lipoprotein patterns in renal transplant patients: A comparison between FK 506 and cyclosporin A patients. Transplant Proc 1998;30:1292-4.

154. Kohnle M, Zimmermann U, Lütkes P, Albrecht K-H, Philipp T and Heemann U. Conversion from cyclosporin A to tacrolimus after kidney transplantation due to hyperlipidemia. Transpl Int 2000;13(S1):S345-8.

155. Margreiter R, Pohanka E, Sparacino V, Sperschneider H, Kunzendorf U, Huber W, Lameire N, Andreucci VE, Donati D and Heemann U for the European Switch to Tacrolimus Study Group. Open prospective multicenter study of conversion to tacrolimus therapy in renal transplant patients experiencing ciclosporin-related side-effects. Transpl Int 2005;18:816-23.

156. Neal B, MacMahon S and Chapman N for the Blood Pressure Lowering Treatment Trialists' Collaboration. Effects of ACE inhibitors, calcium antagonists, and other blood-pressurelowering drugs: results of prospectively designed overviews of randomised trials. Lancet 2000;356:1955-64.

157. Kasiske BL, Anjum S, Shah R, Skogen J, Kandaswamy C, Danielson B, O'Shaughnessy EA, Dahl DC, Silkensen JR, Sahadevan M and Snyder JJ. Hypertension after kidney transplantation. Am J Kidney Dis 2004;43:1071-81.

158. Sijpkens Y, Zwinderman A, Mallat M, Boom H, Fijter J de and Paul L. Intercept and slope analysis of risk factors in chronic renal allograft nephropathy. Graft 2002;5:108-13.

159. Kooman JP, Christiaans MHL, J. Boots, Sande FM van der, Leunissen KML and Hooff JP van. A comparison between office and ambulatory blood pressure measurements in renal transplant patients with chronic transplant nephropathy. Am J Kidney Dis 2001;37:1170-6. 
160. Opelz G and Döhler B for the Collaborative Transplant Study. Improved long-term outcomes after renal transplantation associated with blood pressure control. Am J Transplant 2005;5:2725-31.

161. Artz MA, Boots JMM, Ligtenberg G, Roodnat JI, Christiaans MHL, Hené RJ, Blom HJ, Demacker PNM and Hilbrands LB. Randomized conversion from cyclosporin to tacrolimus in renal transplant patients: Improved lipid profile and unchanged plasma homocystein levels. Transplant Proc 2002;34:1793-4.

162. McCune TR, Thacker LR II, Peters TG, Mulloy L, Rohr MS, Adams PA, Yium J, Light JA, Pruett T, Gaber AO, Selman SH, Jonsson J, Hayes JM, Wright FH jr, Armata T, Blanton J and Burdick JF. Effects of tacrolimus on hyperlipidemia after successful renal transplantation. A Southeastern Organ Procurement Foundation Multicenter Clinical Study. Transplantation 1998;65:87-92.

163. Ross R. Atherosclerosis - An inflammatory disease. N Engl J Med 1999;340:115-26.

164. Keaney JF jr. Atherosclerosis: from lesion formation to plaque activation and endothelial dysfunction. Mol Aspects Med 2000;21:99-166.

165. Venkiteswaran K, Sgoutas DS, Santanam N and Neylan JF. Tacrolimus, cyclosporine and plasma lipoproteins in renal transplant recipients. Transpl Int 2001;14:405-10.

166. Apanay DC, Neylan JF, Ragab MS and Sgoutas DS. Cyclosporin increases the oxidizability of low-density lipoproteins in renal transplant recipients. Transplantation 1994;58:663-9.

167. Morena M, Vela C, Garrigue V, Catanzano J, Descomps B, Cristol JP and Mourad G. Lowdensity lipoprotein composition and oxidation are not influenced by calcineurin inhibitors in renal transplant patients. Transplant Proc 2000;32:2785-6.

168. Varghese Z, Fernando R, Turakhia G, Psimenou E, Brunton C, Fernando ON, Davenport A, Burns A, Sweny P, Powis SH and Moorhead JF. Oxidizability of low-density lipoproteins from neoral and tacrolimus-treated renal transplant patients. Transplant Proc 1998;30:2043-6.

169. Varghese Z, Fernando RL, Turakhia G, Psimenou E, Fernando ON, Sweny P, Powis SH and Moorhead JF. Calcineurin inhibitors enhance low-density lipoprotein oxidation in transplant patients. Kidney Int 1999;56(S71):S137-40.

170. Martinez Castelao A, Ramos R, Seron D, Gil-Vernet S, Fiol C, Gomez-Gerique N, Yzaguirre MT, Hurtado I, Sabate I, Alsina J and Grinyo JM. Effect of cyclosporin and tacrolimus on lipoprotein oxidation after renal transplantation. Nefrologia 2002;22:364-9.

171. Wissing KM, Abramowicz D, Broeders $\mathrm{N}$ and Vereerstraeten P. Hypercholesterolemia is associated with increased kidney graft loss caused by chronic rejection in male patients with previous acute rejection. Transplantation 2000;70:464-72.

172. Wilson PWF, D'Agostino RB, Levy D, Belanger AM, Silbershatz H and Kannel WB. Prediction of coronary heart disease using risk factor categories. Circulation 1998;97:1837-47.

173. Ducloux D, Kazory A and Chalopin JM. Predicting coronary heart disease in renal transplant recipients: a prospective study. Kidney Int 2004;66:441-7.

174. Meier-Kriesche H-U, Baliga R and Kaplan B. Decreased renal function is a strong risk factor for cardiovascular death after renal transplantation. Transplantation 2003;75:1291-5.

175. Pierrat A, Gravier E, Saunders C, Caira MV, Ait-Djafer Z, Legras B and Mallie JP. Predicting GFR in children and adults: a comparison of the Cockcroft-Gault, Schwartz, and modification of diet in renal disease formulas. Kidney Int 2003;64:1425-36.

176. Hariharan S, McBride MA, Cherikh WS, Tolleris CB, Bresnahan BA and Johnson CP. Posttransplant renal function in the first year predicts long-term kidney transplant survival. Kidney Int 2002;62:311-8. 
177. Kaplan B, Schold J and Meier-Kriesche H-U. Poor predictive value of serum creatinine for renal allograft loss. Am J Transplant 2003;3:1560-5.

178. Kasiske BL, Andany MA and Danielson B. A thirty percent chronic decline in inverse serum creatinine is an excellent predictor of late renal allograft failure. Am J Kidney Dis 2002;39: $762-8$.

179. Ojo AO, Held PJ, Port FK, Wolfe RA, Leichtman AB, Young EW, Arndorfer J, Christensen L and Merion RM. Chronic renal failure after transplantation of a nonrenal organ. $\mathrm{N}$ Engl J Med 2003;349:931-40.

180. Randhawa PS, Shapiro R, Jordan ML, Starzl TE and Demetris AJ. The histopathological changes associated with allograft rejection and drug toxicity in renal transplant recipients maintained on FK506. Clinical significance and comparison with cyclosporine. Am J Surg Pathol 1993;17:60-8.

181. Mihatsch MJ, Kyo M, Morozumi K, Yamaguchi Y, Nickeleit V and Ryffel B. The side effects of ciclosporin-A and tacrolimus. Clin Nephrol 1998;49:356-63.

182. Solez K, Vincenti F and Filo RS. Histopathologic findings from 2-year protocol biopsies from a U.S. multicenter kidney transplant trial comparing tacrolimus versus cyclosporine. A report of the FK506 Kidney Transplant Study Group. Transplantation 1998;66:1736-40.

183. Duijnhoven EM van, Dackus HJA, Christiaans MHL, Heurn ELW van and Hooff JP van. Comparable renal function in living kidney donors and their recipients on tacrolimus-based immunosuppression. ICI San Diego 2004;Abstract P-54.

184. Kaplan B, Schold JD and Meier-Kriesche H-U. Long-term graft survival with Neoral and tacrolimus: A paired kidney analysis. J Am Soc Nephrol 2003;14:2980-4.

185. Cheung CYS, Wong KM, Chan HW, Liu YL, Chan YH, Wong HS, Chak WL, Choi KS, Chau $\mathrm{KF}$ and Li CS. Paired kidney analysis of tacrolimus and cyclosporine microemulsion-based therapy in Chinese cadaveric renal transplant recipients. Transpl Int 2006;19:657-66.

186. Larson TS, Dean PG, Stegall MD, Griffin MD, Textor SC, Schwab TR, Gloor JM, Cosio FG, Lund WJ, Kremers WK, Nyberg SL, Ishitani MB, Prieto M and Velosa JA. Complete avoidance of calcineurin inhibitors in renal transplantation: A randomized trial comparing sirolimus and tacrolimus. Am J Transplant 2006;6:514-22.

187. Ferraris JR, Tambutti ML, Cardoni RL and Prigoshin N. Conversion from cyclosporine A to tacrolimus in paediatric kidney transplant recipients with chronic rejection: changes in the immune responses. Transplantation 2004;77:532-7.

188. Cantarovich D, Renou M, Megnigbeto A, Giral-Classe M, Hourmant M, Dantal J, Blancho G, Karam G and Soulillou JP. Switching from cyclosporine to tacrolimus in patients with chronic transplant dysfunction or cyclosporine-induced adverse events. Transplantation 2005;79:72-8.

189. Hohage H, Welling U, Zeh M, Gerhardt U and Suwelack B. Switching immunosuppression from cyclosporine to tacrolimus improves long-term kidney function: a 6-year study. Transplant Proc 2005;37:1898-9.

190. Meier M, Nitschke M, Weidtmann B, Jabs WJ, Wong W, Suefke S, Steinhoff J and Fricke L. Slowing the progression of chronic allograft nephropathy by conversion from cyclosporine to tacrolimus: A randomized controlled trial. Transplantation 2006;81:1035-40.

191. Hunsicker LG, Adler S, Caggiula A, England BK, Greene T, Kusek JW, Rogers NL and Teschan PE. Predictors of the progression of renal disease in the Modification of Diet in Renal Disease Study. Kidney Int 1997;51:1908-19.

192. Halloran PF. Call for revolution: a new approach to describing allograft deterioration. Am J Transplant 2002;2:195-200. 
193. Gill JS, Tonelli M, Mix CH, Johnson N and Pereira BJG. The effect of maintenance immunosuppression medication on the change in kidney allograft function. Kidney Int 2004;65:692-9.

194. Jain S, Bicknell GR and Nicholson ML. Tacrolimus has less fibrinogenic potential than cyclosporin A in a model of renal ischaemia-reperfusion injury. Br J Surg 2000;87:1563-1568

195. Esposito C, Foschi A, Parrilla B, Cornacchia F, Fasoli G, Plati AR, De Mauri A, Mazzullo T, Scudellaro $\mathrm{R}$ and Dal Canton A. Effect of calcineurin inhibitors on extracellular matrix turnover in isolated human glomeruli. Transplant Proc 2004;36:695-7.

196. Bicknell GR, Shaw WJA, Pringle JH, Furness PN and Nicholson ML. Differential effects of cyclosporin and tacrolimus on the expression of fibrosis-associated genes in isolated glomeruli from renal transplants. Br J Surg 2000;87:1569-75.

197. Mohamed MAS, Robertson H, Booth TA, Balupuri S, Kirby JA and Talbot D. TGF- $\beta$ expression in renal transplant biopsies. A comparative study between cyclosporin- $A$ and tacrolimus. Transplantation 2000;69:1002-5.

198. Jain S, Mohamed MA, Sandford R, Furness PN, Nicholson ML and Talbot D. Sequential protocol biopsies from renal transplant recipients show an increasing expression of active TGF beta. Transpl Int 2002;15:630-4.

199. Khanna A, Plummer M, Bromberek C, Bresnahan B and Hariharan S. Expression of TGF- $B$ and fibrogenic genes in transplant recipients with tacrolimus and cyclosporine nephrotoxicity. Kidney Int 2002;62:2257-63.

200. Nankivell BJ, Borrows RJ, Fung CL-S, O'Connell PJ, Chapman JR and Allen RDM. Delta analysis of posttransplantation tubulointerstitial damage. Transplantation 2004;78:434-41.

201. Murphy GJ, Waller JR, Sandford RS, Furness PN and Nicholson ML. Randomized clinical trial of the effect of microemulsion cyclosporin and tacrolimus on renal allograft fibrosis. Br J Surg 2003;90:680-6.

202. Roos-van Groningen MC, Scholten EM, Lelieveld PM, Rowshani AT, Baelde HJ, Bajema IM, Florquin S, Bemelman FJ, Heer E de, Fijter JW de, Bruijn JA and Eikmans M. Molecular comparison of calcineurin inhibitor-induced fibrogenic responses in protocol renal transplant biopsies. J Am Soc Nephrol 2006;17:881-8.

203. Hooff JP van, Walt I van der, Kallmeyer J, Miller D, Dawood S, Moosa M and Undre N. Conversion of stable kidney transplant recipients from a twice daily prograf based regimen to a once daily modified release tacrolimus based regimen. ICI San Diego 2004;abstract 26.

204. Hardinger KL, Park JM, Schnitzler MA, Koch MJ, Miller BW and Brennan DC. Pharmacokinetics of tacrolimus in kidney transplant recipients: twice daily versus once daily dosing. Am J Transplant 2004;4:621-5.

205. Teutonico A, Schena PF and Di Paolo S. Glucose metabolism in renal transplant recipients: effect of calcineurin inhibitor withdrawal and conversion to sirolimus. J Am Soc Nephrol 2005;16:3128-35. 
Abbreviations 


\section{Abbreviations}

ACE

$\mathrm{ACTH}$

ADA

ADMA

ANCOVA

ANOVA

AP

ATG

AUC

AZA

BMI

cAMP

CAN

CETP

cGMP

CIT

CMV

$\mathrm{CNI}$

COX

CREB

CsA

CYP3A4

DDAH

DM

DNA

ELAM

eNOS

ESRD

ET

FFA

FKBP

GFR

GM-CSF

GRE

$\mathrm{Hb}$

$\mathrm{HB}$

HDL

HDL-c

HLA

HMG-coA
Angiotensin Converting Enzyme

Adrenocorticotrophic Hormone

American Diabetes Association

Asymmetric Dimethyl-Arginine

Analysis of Co-Variance

Analysis of Variance

Activator Protein

Anti-Thymocyte Globulin

Area Under the Curve

Azathioprine

Body Mass Index

cyclic Adenosine-Monophosphate

Chronic Allograft Nephropathy

Cholesteryl-Ester Transfer Protein

cyclic Guanylate-Monophosphate

Cold Ischemia Time

Cytomegalovirus

Calcineurin Inhibitor

Cyclooxygenase

c-AMP-Response Element-Binding Protein

Cyclosporin A

Cytochrome P450 subtype 3A4

Dimethylarginine-Dimethyl-Amino-Hydrolase

Diabetes Mellitus

Deoxyribonucleic Acid

Endothelial-Leucocyte Adhesion Molecule

endothelial Nitric Oxide Synthase

End-Stage Renal Disease

Endothelin

Free Fatty Acid

FK-Binding Protein

Glomerular Filtration Rate

Granulocyte-Macrophage Colony Stimulating Factor

Glucocorticoid Response Element

Hemoglobin

Heart Beating

High Density Lipoprotein

High Density Lipoprotein-cholesterol

Human Leucocyte Antigen

3-Hydroxy-3-Methyl-Glutaryl-coenzyme A 


$\begin{array}{ll}\text { HOMA } & \text { Homeostasis Model Assessment } \\ \text { HR } & \text { Hazard Ratio } \\ \text { HSL } & \text { Hormone Sensitive Lipase } \\ \text { HUS } & \text { Hemolytic Uremic Syndrome } \\ \text { ICAM } & \text { Intracellular Adhesion Molecule } \\ \text { IDL-c } & \text { Intermediate Density Lipoprotein-cholesterol } \\ \text { IFN } & \text { Interferon } \\ \text { IL } & \text { Interleukin } \\ \text { IMT } & \text { Intima-Media Thickness } \\ \text { iNOS } & \text { inducible Nitric Oxide Synthase } \\ \text { iv-GTT } & \text { intravenous Glucose Tolerance Test } \\ \text { ITT } & \text { Insulin Tolerance Test } \\ \text { JNK } & \text { Jun N-terminal Kinase } \\ \text { kG } & \text { Glucose disappearance rate }=\text { insulin sensitivity index } \\ \text { LDL-c } & \text { Low Density Lipoprotein cholesterol } \\ \text { Lp(a) } & \text { Lipoprotein (a) } \\ \text { LPL } & \text { Lipoprotein Lipase } \\ \text { LRD } & \text { Living Related Donor } \\ \text { LUD } & \text { Living Unrelated Donor } \\ \text { LVH } & \text { Left Ventricular Hypertrophy } \\ \text { MAPK } & \text { Mitogen-Activated Protein Kinases } \\ \text { MCP } & \text { Monocyte Chemotactic Protein } \\ \text { MDR } & \text { Multi Drug Resistance } \\ \text { MMF } & \text { Mycophenolate Mofetil } \\ \text { mRNA } & \text { messenger Ribonucleic Acid } \\ \text { MTHFR } & \text { Methylene-Tetrahydrofolate Reductase } \\ \text { MTSOSDS } & \text { Modified Transplant Symptom Occurrence and Distress Scale } \\ \text { NCEP } & \text { National Cholesterol Education Program } \\ \text { NF- } x \text { B } & \text { Nuclear Factor kappa B } \\ \text { NO } & \text { Nitric Oxide } \\ \text { NOS } & \text { Nitric Oxide Synthase } \\ \text { o-GTT } & \text { oral Glucose Tolerance Test } \\ \text { OR } & \text { Odds Ratio } \\ \text { Ox-LDL } & \text { Oxidized Low Density Lipoprotein } \\ \text { PAI } & \text { Plasminogen Activator Inhibitor } \\ \text { P-gP } & \text { P-glycoprotein } \\ \text { PRA } & \text { Panel Reactive Antibodies } \\ \text { PTDM } & \text { Post-Transplant Diabetes Mellitus } \\ \text { PTH } & \text { Parathyroid Hormone } \\ \text { r-ATG } & \text { rabbit-Anti-Thymocyte Globulin } \\ \text { RAPA } & \text { Rapamycine, sirolimus } \\ \text { RR } & \text { Relative Risk } \\ & \end{array}$


SEM

SOD

$\mathrm{SD}$

SST

STAT

TAC

TG

TGF

TNF

tPA

TOR

TTP

Tx

UNOS

uPA

USRDS

VCAM

VLDL

vWF

WHO

WIT

Standard Error of the Mean

Superoxide Dismutase

Standard Deviation

Short Synacthen Test

Single Transducers and Activators of Transcription

Tacrolimus

Triglycerides

Transforming Growth Factor

Tumour Necrosis Factor

tissue-type Plasminogen Activator

Target of Rapamycin

Thrombotic Thrombocytopenic Purpura

Transplantation

United Network for Organ Sharing

urokinase-type Plasminogen Activator

United States Renal Data System

Vascular Cell Adhesion Molecule

Very Low Density Lipoprotein

von Willebrand Factor

World Health Organisation

Warm Ischemia Time 
Samenvatting 


\section{Samenvatting}

Als een lichaamsvreemd orgaan, zoals een nier, getransplanteerd wordt, zal het afweersysteem moeten worden geremd om acute afstoting (rejectie) tegen te gaan. In het begin van de jaren negentig was cyclosporine A (CsA) het belangrijkste medicament na een niertransplantatie. CsA is een remmer van het in de cel gelegen eiwit calcineurine dat in de celkern van afweercellen de afgifte van bepaalde eiwitten, die de afstotingsreactie stimuleren, regelt. In de eerste helft van de jaren negentig werd ook een nieuw medicament ontwikkeld, tacrolimus (TAC), dat een sterkere remming van calcineurine bleek te bewerkstellen dan CsA. In de eerste grote studies bleek TAC beter in staat tot het voorkomen van acute rejecties dan CsA, met een vergelijkbaar effect op de patiënt- en niertransplantaatoverleving. Daarnaast waren er verschillen in bijwerkingen ook op het gebied van hart- en vaatziekten. Hart- en vaatziekten komen frequent voor bij en zijn een belangrijke oorzaak van sterfte van patiënten met een gestoorde nierfunctie zoals dialyse- en niertransplantatiepatiënten. Met CsA behandelde patiënten hadden hogere bloedwaardes voor het cholesterolgehalte. Met TAC ontwikkelden meer patiënten diabetes mellitus (suikerziekte) na de transplantatie, die overigens in een groot gedeelte omkeerbaar bleek.

In 1995 werd een nieuwe preparaatvorm van CsA geïntroduceerd. Deze microemulsie vorm $\left(\mathrm{Neoral}^{\circledR}\right)$ gaf een stabielere en beter voorspelbare opname van CsA uit de darm. Met dit middel was het risico op een acute afstoting lager dan met de oorspronkelijke formulering. Nadien ontstond discussie of het voordeel van TAC op het risico van acute afstoting nog wel aanwezig was ten opzichte van deze nieuwe micro-emulsie vorm van CsA. Omdat prospectieve (vooruitkijkende) studies ontbraken, hebben we alle met TAC behandelde patiënten in de periode tussen 1995 en 1997 retrospectief (terugkijkend) geanalyseerd en vergeleken met alle met CsA behandelde patiënten in deze zelfde periode. Omdat TAC in die periode nog niet als geneesmiddel geregistreerd was in Nederland, bestond de groep patiënten die TAC gekregen hadden voor een aanzienlijk gedeelte uit patiënten met een relatief hoog risico op een acute afstoting. Desondanks bleek in onze studie, waarvan de resultaten beschreven zijn in Hoofdstuk 2, dat TAC significant (met statistiek bewezen) minder acute rejecties tot gevolg had dan de nieuwe micro-emulsie vorm van CsA. Daarnaast bleek ook het aantal ernstige afstotingsreacties (afstotingsreacties, die niet goed reageren op hoge doseringen corticosteroïden en die een slechte prognose voor de functie van de getransplanteerde nier inhouden) verminderd. Ook bleek TAC in staat tot het omkeren van acute afstotingen bij patiënten, die tevoren met CsA werden behandeld. Deze resultaten werden nadien bevestigd in prospectief gerandomiseerde studies en meta-analyses. Ook bleek het door de betere preventie van acute afstotingsreacties mogelijk om begeleidende medicatie in een groot 
gedeelte van de patiënten af te bouwen en $70 \%$ van de patiënten te behandelen met slechts één medicament om afstoting tegen te gaan. Een belangrijk medicament dat kon worden afgebouwd was prednison of prednisolon, zogenaamde corticosteroïden. Corticosteroïden waren de eerste bekende geneesmiddelen om afweerreacties te onderdrukken en hebben vanaf het begin van het uitvoeren van transplantaties altijd een belangrijke rol gespeeld. Corticosteroïden werken niet erg specifiek en kennen bij langdurig gebruik nogal wat bijwerkingen. Desondanks werden ze altijd erg belangrijk geacht om het risico op een acute afstoting te verminderen. Het stoppen van corticosteroïden bij het gebruik van TAC leidde echter niet tot het optreden van acute rejecties, zoals dat met CsA wel bij 5-10\% bleek op te treden. Verder bleek het bijwerkingenprofiel van TAC gunstiger met lagere waardes voor het cholesterolgehalte in het bloed en een goede bloeddrukregulatie met minder medicijnen. Het risico op diabetes mellitus was in de initiële fase na de transplantatie hoger met TAC, maar na verlaging van de dosering van TAC en het stoppen van corticosteroïden bleek het optreden van diabetes vergelijkbaar met het optreden tijdens behandeling met CsA.

Zoals eerder gezegd hebben corticosteroïden op de lange termijn een flink aantal bijwerkingen. Zij verhogen het risico op diabetes mellitus, hypertensie (hoge bloeddruk) en hypercholesterolemie (een verhoogd cholesterolgehalte) en indirect hiermee het risico op hart- en vaatziekten. Verder geven ze aanleiding tot overgewicht en een toename van vet afgezet in de buik, botontkalking (osteoporose), staar (cataract) en een verhoogde oogboldruk (glaucoom). Daarnaast geven ze ook nog een dunnere huid en bloedvatwand en kunnen ze psychiatrische stoornissen veroorzaken. Gezien deze bijwerkingen kan het soms gewenst zijn om corticosteroïden snel te stoppen. Helaas onderdrukken corticosteroïden de eigen bijnier, die normaal een dergelijke stof in kleine hoeveelheden aanmaakt. Het snel stoppen van corticosteroïden kan dan gevaarlijk zijn, omdat het lichaam dan nog niet in staat is de eigen productie weer op gang te brengen. In bepaalde situaties, met name in perioden van stress, kan dit aanleiding geven tot levensbedreigende lichamelijke verschijnselen. Om het risico op dit soort ongewenste effecten nader in te schatten hebben we in Hoofdstuk 3 een studie gedaan waarbij een groep patiënten werd gevolgd, die volgens een vooropgezet protocol na drie of zes maanden werden ingeschaald om in twee weken tijd te stoppen met hun dosering corticosteroïden (10 mg prednison). Vóór het stoppen van de corticosteroïden werd hun bijnier gestimuleerd met een zogenaamde korte synacthen test. Hieruit bleek dat ongeveer $50 \%$ van de patiënten enige vorm van onderdrukking had van zijn bijnier. Desondanks konden de corticosteroïden na drie maanden zonder problemen in twee weken worden gestopt. $\mathrm{Na}$ zes maanden traden bij $30 \%$ van de patiënten echter klachten op, die te maken zouden kunnen hebben met een onvoldoende functionerende bijnier. 
Een gestoorde korte synacthen test bleek geen goede voorspeller om patiënten, die klachten gingen ontwikkelen op te sporen. Wel konden patiënten worden geselecteerd, waarbij de snelle afbouw van corticosteroïden veilig kon worden toegepast. Hierbij werd eerst 's ochtends een bloedmonster afgenomen voor bepaling van de eigen productie van corticosteroïden (cortisol). Indien deze cortisolwaarde $>400 \mathrm{nmol} / \mathrm{l}$ bedroeg, was versneld afbouwen veilig. Indien de ochtend cortisolwaarde $<400 \mathrm{nmol} / 1$ bedroeg, voorspelde een goede respons op de korte synacthen test (cortisolwaarde na stimulatie $>550 \mathrm{nmol} / \mathrm{l}$ ) een veilige versnelde afbouw van de corticosteroïden.

Een opvallende observatie die we deden tijdens onze studies met het afbouwen van de corticosteroïden was het stijgen van de bloedspiegels van TAC. In Hoofdstuk 4 hebben we dit gegeven zowel retrospectief als prospectief nader onderzocht. Het bleek dat de dosisgenormaliseerde waarde (bloedspiegel van TAC vóór een nieuwe toediening (dalspiegel) gedeeld door de corresponderende dosis van TAC) met 10-15\% toenam na staken van $5 \mathrm{mg}$ prednison en met ongeveer $35 \%$ na het staken van $10 \mathrm{mg}$ prednison. In een farmacokinetisch gedeelte (Farmacokinetiek is de wetenschap die onderzoekt hoe snel een medicament in het lichaam wordt opgenomen en weer wordt uitgescheiden) kon prospectief een $18 \%$ toename worden vastgesteld in de expositie (blootstelling) aan TAC na stoppen van $5 \mathrm{mg}$ prednison. Omdat corticosterö̈den een bepaald eiwitsysteem in de lever stimuleren (cytochroom P450, met name subtype 3A4) en TAC juist door dit eiwit wordt afgebroken, betekent dit dat corticosteroïden de afbraak van TAC versnellen. Hierdoor is er meer TAC nodig om eenzelfde bloedspiegel en dus effect te bereiken. Dit heeft weer tot gevolg dat de kosten voor de behandeling stijgen. Bovendien is een bijwerking van TAC bij hogere bloedspiegels een verslechtering van de nierfunctie. Door de hogere bloedspiegels na het afbouwen van de corticosteroïden kan de nierfunctie dus achteruitgaan en verkeerd beoordeeld worden als een acute afstoting.

Nadat ons gebleken was dat corticosterö̈den veilig konden worden afgebouwd na de eerste drie tot zes maanden na de transplantatie en ook zo nodig relatief snel konden worden gestopt, stelden we ons de vraag of de corticosterö̈den ook vroeg na de transplantatie konden worden gestopt. Hiervoor ontwikkelden we een oriënterende studie, waarbij prospectief in de ene groep de corticosterö̈den vroeg (10 e dag) na de transplantatie werden gestopt, mits de bloedspiegel van TAC stabiel het gewenste niveau had bereikt, en in de andere groep de corticosteroïden in drie maanden geleidelijk werden uitgeslopen, zoals onze standaard behandeling was. In deze studie bleek, zoals beschreven in Hoofdstuk 5, dat het vroeg stoppen van de corticosterö̈den niet leidde tot een hoger aantal afstotingen. Opvallend was dat de afstotingsreacties juist wat later optraden. Onze interpretatie was dat dit waarschijnlijk kwam door een stijging van de bloedspiegels van TAC na het 
stoppen van de corticosteroïden. Deze stijging hebben we in de vorige paragraaf al uitgelegd en uitvoering beschreven in Hoofdstuk 4.

Transplantaatfunctie één jaar na de transplantatie en eiwitverlies in de urine vormen belangrijke voorspellers voor de levensduur van een getransplanteerde nier. In onze studie konden geen verschillen worden aangetoond tussen beide behandelingsgroepen wat betreft deze parameters. Tegenwoordig hebben meerdere studies waarin de corticosteroïden zijn gestopt vergelijkbare langere termijn resultaten laten zien, zonder een eerder gemeld risico van chronische afstoting, zoals bij met CsA behandelde patiënten.

Een opvallende bijkomende observatie was dat door het vroeg stoppen met de corticosteroïden veel minder patiënten diabetes mellitus ontwikkelden na de transplantatie. Later toonden meerdere en grotere studies vergelijkbare resultaten.

Omdat zowel TAC als corticosteroïden een rol speelden bij het ontwikkelen van diabetes mellitus na een transplantatie, zijn we in Hoofdstuk 6 op zoek gegaan naar de relatieve effecten van beide middelen op de glucose-(bloedsuiker)stofwisseling. Hiervoor ondergingen 15 niertransplantatiepatiënten een glucose belastingstest door middel van een infuus (iv-GTT: intraveneuze glucose tolerantie test). Vervolgens werd deze test herhaald na het stoppen van de corticosteroïden (10 mg prednison) en nogmaals na verlaging van de bloedspiegel van TAC naar onderhoudswaardes. Uit onze studie bleek dat corticosteroïden geen effect hadden op de afgifte van insuline, maar wel de gevoeligheid van de lichaamscellen voor dit hormoon deden verminderen en dat TAC juist de insulineafgifte remde, maar geen effect had op de gevoeligheid voor insuline. De insulinegevoeligheidsindex $\left(\mathrm{k}_{\mathrm{g}}\right)$, een resultante van zowel de insulineafgifte als de insulinegevoeligheid, verbeterde uitsluitend na het stoppen van de corticosteroïden. Dit suggereert dat de corticosteroïden mogelijk een groter belang hebben in het optreden van diabetes mellitus na de transplantatie dan TAC. De observatie uit Hoofdstuk 5 met het lage optreden van diabetes bij het vroeg stoppen van de corticosteroïden onderschrijft dit.

TAC had dus voordelen ten opzichte van CsA als basismiddel om afstoting tegen te gaan bij nieuwe transplantaties, gezien minder rejecties en een gunstiger risicoprofiel voor hart- en vaatziekten, waarbij het risico van diabetes was op te vangen door het vroeg afbouwen van corticosteroïden en eventueel TAC dosisvermindering. Onduidelijk was echter of transplantatiepatiënten die behandeld worden met CsA als basismiddel en die een stabiele functie van het transplantaat hebben ook nog zouden kunnen profiteren van het gunstigere risicoprofiel voor hart- en vaatziekten van TAC. Hiertoe werd een multi-center studie opgezet met de transplantatiecentra in Nijmegen, Maastricht, Utrecht en Rotterdam. In totaal namen 124 patiënten deel aan deze studie waarbij de helft werd geloot tot een omzetting (conversie) naar TAC als basismiddel en de andere 
helft CsA bleef gebruiken. In Hoofdstuk 7 worden de resultaten van de eerste zes maanden gepresenteerd. Allereerst bleek conversie veilig. Er traden geen rejecties op. Verder resulteerde TAC inderdaad in een gunstiger risicoprofiel voor hart- en vaatziekten met een daling van de bloeddruk, een daling van zowel het totale als het ongunstige lage densiteit lipoproteïne (LDL)-cholesterol, een daling in het vetgehalte (triglyceride), een verbetering in de oxidatie van het LDL, en een afname in de hoogte van het gehalte aan fibrinogeen (een eiwit dat een rol speelt bij de bloedstolling en ook geassocieerd is met het optreden van hart- en vaataandoeningen). Er bleek bovendien geen sprake van een verhoogd voorkomen van diabetes mellitus. Verrassend bleek echter een verbetering in de transplantaatfunctie met een daling van het creatininegehalte als resultante van de nierfunctie in het bloed. In Hoofdstuk 8 zijn de 2-jaars resultaten van deze studie gepresenteerd. In deze studie bleek dat de resultaten na zes maanden voortduurden tot twee jaar na de interventie. Bovendien was er sprake van een geleidelijke achteruitgang van de niertransplantaatfunctie in de groep die CsA was blijven gebruiken terwijl er sprake was van een stabiele niertransplantaatfunctie in de groep die omgezet was naar TAC. Uit een vragenlijst gericht op bijwerkingen van beide middelen bleek bovendien dat de patiënten het gebruik van TAC beter waardeerden. Latere omzettingstudies bevestigden de gunstige resultaten op het gebied van de risicofactoren voor hart- en vaatziekten en de niertransplantaatfunctie.

Tegenwoordig worden meer medicijnen als onderhoudsmedicatie gebruikt om afstoting tegen te gaan bij niertransplantatiepatiënten. In Hoofdstuk 9 is een uitgebreide literatuurevaluatie weergegeven van alle effecten van alle gangbare onderhoudsmedicamenten op het gebied van hart- en vaatziekten. Hierbij moet in acht worden genomen, dat onderhoudsmedicatie meer behelst dan alleen een gunstig risicoprofiel voor hart- en vaatziekten. Bovenal moet afstoting worden tegengegaan. Calcineurine remmers, zoals CsA en TAC, hebben het sterkste afstotingsremmende effect. Echter beide middelen kunnen aanleiding geven tot nierschade. Zoals al eerder in dit hoofdstuk aangegeven en uitgebreid beschreven in Hoofdstuk 9, heeft TAC een gunstiger risicoprofiel voor hart- en vaatziekten dan CsA. Ook komen er steeds meer aanwijzingen dat het nierbeschadigende effect van TAC minder uitgesproken is dan dat van CsA.

Corticosteroïden zijn erg ongunstig ten opzichte van hart- en vaatziekten, zoals al eerder ook in dit hoofdstuk aangegeven. Sirolimus (Rapamycine) is een nieuwer middel dat als belangrijkste voordeel een mogelijk remmend effect heeft op de ontwikkeling van vaatschade. Echter het geeft ook aanleiding tot een forse stijging van het cholesterol- en triglyceridegehalte. Het precieze effect op het optreden van hart- en vaatziekten dient nog te worden vastgesteld. Daarnaast zijn vaak andere medicamenten erbij nodig om afstoting tegen te gaan. Hierbij wordt vaak weer gebruikt gemaakt van corticosteroïden, die juist weer ongunstig zijn. 
Mycophenolaat mofetil $\left(\right.$ Cellcept $\left.^{\mathbb{R}}\right)$ is een middel dat weinig bijwerkingen heeft op het gebied van hart- en vaatziekten. Helaas is het minder krachtig om afstoting tegen te gaan dan calcineurine remmers, waardoor weer aanvullende medicatie nodig is. De belangrijkste bijwerking op het gebied van hart- en vaatziekten is bloedarmoede wat een belasting geeft voor het hart en een verdikking van de hartspier kan geven. Op dit moment ontbreken nog voldoende lange termijn gegevens om de precieze waarde vast te stellen.

Concluderend laat dit proefschrift zien dat TAC niet alleen een krachtiger middel is om afstotingsreacties tegen te gaan dan CsA, maar dat het ook een gunstiger risicoprofiel heeft voor hart- en vaatziekten. Het risico op diabetes mellitus kan geminimaliseerd worden door het vroeg afbouwen van corticosteroïden en dosisvermindering van TAC. Met betrekking tot het optreden van diabetes geeft TAC remming van de afgifte van insuline en geven corticosteroïden een verlaging van de gevoeligheid voor insuline. Daarnaast geeft TAC in gebruikelijke onderhoudsdoseringen een laag risico op nierschade. Door zijn krachtige werking is het goed mogelijk om corticosteroïden met hun vele bijwerkingen te stoppen en een groot gedeelte te behandelen met slechts één medicament tegen afstoting. Bij het stoppen van corticosteroïden moet rekening gehouden worden met een interactie met TAC, waardoor hogere bloedspiegels de transplantaatfunctie kunnen verslechteren en een afstotingsreactie kunnen nabootsen. Daarnaast bestaat er een klein risico bij het snel afbouwen van corticosteroïden na de eerste drie maanden na de transplantatie op verschijnselen van een onvoldoende herstel van de eigen bijnierfunctie.

Verder is aangetoond dat niet alleen patiënten die starten met TAC vanaf de transplantatie profiteren van dit middel, maar ook dat omzetting van met CsA behandelde patiënten resulteert in een gunstiger risico op hart- en vaatziekten en een betere bescherming van de niertransplantaatfunctie en dat patiënten zelf TAC prefereren boven CsA. 
Dankwoord 


\section{Dankwoord}

Zoals iedereen zal begrijpen, komt een proefschrift nooit vanzelf tot stand. Zonder de hulp van menigeen had ik dit proefschrift dan ook nooit kunnen voltooien. In dit hoofdstuk wil ik iedereen dan ook nadrukkelijk bedanken, die zijn steentje aan dit werk heeft bijgedragen. Hoewel ik velen bij naam zal noemen, kan het misschien toch nog voorkomen dat ik iemand niet persoonlijk noem. Hierbij wil ik al diegenen laten weten dat dit geen opzettelijke omissie betreft, maar dat ook naar hen mijn dank uitgaat.

In de eerste plaats komt een proefschrift nooit tot stand zonder de steun van het thuisfront. Lieve Sabine, wij hebben elkaar leren kennen tijdens het uitvoeren van een groot deel van het in dit proefschrift beschreven onderzoek. Daardoor weet je als geen ander, hoe groot de last is, die zo'n proefschrift legt op de thuissituatie. Ik wil je bij deze bedanken voor alle steun en opoffering die jij in al die jaren gegeven hebt. Ik hoop nu meer rust en tijd te vinden niet alleen voor jou, maar ook om samen ons aanstaande kind op te voeden.

Mijn promotor, Prof. dr. J.P. van Hooff, beste Hans, jij zag talenten in mij, die anderen op dat moment niet herkenden. Hoewel het begin nog wel eens stroef verliep, wist je mij altijd weer te motiveren om door te gaan. $\mathrm{Nu}$ ben ik blij dat we het kunnen afronden. Jij zag al vroeg de capaciteiten van tacrolimus en de nadelen van corticosteroïden, waarbij je oog had voor de hele patiënt en niet alleen zijn transplantaatfunctie. Dit heb ik altijd zeer gewaardeerd. Bijzonder waardeer ik ook jouw visie op het presenteren van de boodschap. Telkens weer wist je mijn ingebrachte stukken te verbeteren. Voor alles mijn hartelijke dank.

Mijn copromotor, Dr. M.H.L. Christiaans, beste Maarten, jouw kennis van de statistiek en in het bijzonder de survivalanalyses was onontbeerlijk voor het tot stand brengen van dit proefschrift. Daarnaast vond je altijd tijd voor mij ondanks je veelzijdige takenpakket. Alleen door je enthousiasme merkte je niet altijd dat ik met verkleumde handen achter de computer zat. Verder heeft je besliskracht in het onderlinge overleg een doorslaggevende rol gespeeld. Voor alles mijn hartelijke dank.

Elly van Duijnhoven, beste Elly, de een zijn dood, de ander... Doordat jij je aanvankelijk terugtrok uit het onderzoek, kreeg ik de gelegenheid ermee te starten. Gelukkig heb jij je volledig herpakt en ben je inmiddels ruim voor mij gepromoveerd. Ik wil je in het bijzonder danken voor alle steun die je altijd hebt gegeven en de bereidwilligheid mee te werken aan een groot deel van het uitgevoerde onderzoek. Verder vond ik bij jou altijd een gewillig oor als het een of ander weer eens tegen zat. 
De overige leden van de werkgroep nefrologie: Prof. dr. K.M.L. Leunissen, Dr. J.P. Kooman en Dr. F.M. van de Sande, beste Karel, Jeroen en Frank, hartelijk dank voor de zeer aangename werksfeer die altijd heerste op de nefrologie. Dank ook voor jullie begeleiding in de dialyse en klinische nefrologie. Ik kon bij problemen altijd bij jullie terecht. Jeroen, jij vooral ook bedankt voor de vena cava echo's. Ik maak er nog steeds met veel plezier gebruik van.

Alle nefrologen in opleiding. In de eerste plaats gaat mijn dank uit naar Stijn Konings en Charles Beerenhout. Mede door jullie samenwerking heb ik het altijd erg naar mijn zin gehad op de afdeling nefrologie. Jullie plek in de kelder was altijd een prettige uitvlucht. Dankzij jullie verwar ik ook geen mededelingen meer met de rondvraag. Stijn met name ook bedankt voor de lessen peritoneaal dialyse. Liesbeth Hendrickx, hoewel je slechts korte tijd te gast was, heb je toch een belangrijke rol gespeeld en heeft mijn leven mede hierdoor een bijzondere wending gekregen. Pieter van Paassen, onnavolgbaar waren je uiteenzettingen over diverse onderwerpen, tegenwoordig ben ik je ook dank verschuldigd voor je adviezen voor de behandeling van vasculitis patiënten. Mariëlle Gelens, je hebt mijn plekje overgenomen. Ik wil je vooral ook veel succes wensen met de voortzetting. Willie van Kuijk, Mariëlle Krekels, Marc Hermans, Martine Verhoeven, Dirk Scheepers en Tom Dejagere, jullie wil ik bedanken voor de steun en de goede samenwerking.

Monique Mullens, jij als onderzoeksassistente was onmisbaar voor het uitvoeren van alle metingen en het maken van alle afspraken. Hiervoor hartelijk dank.

Eugenie van den Ham, jou ben ik veel dank verschuldigd voor het organiseren en grotendeels uitvoeren van alle synacthen testen. Daarnaast waren je statistische adviezen ook een welkome bijdrage. Ook jij hartelijk bedankt.

Secretariaat Nefrologie, beste Etienne, Anke en Marleen, jullie waren van uitzonderlijk belang voor het opsporen van verdwenen groene lijsten. Verder kon ik altijd met diverse vragen bij jullie terecht. Voor alles hartelijk dank.

Medewerkers afdeling D5, jullie steun en hulp was eveneens van wezenlijk belang. Zonder jullie had ik alle benodigdheden nooit gevonden. John Dackus in het bijzonder wil ik bedanken voor de hulp bij het uitvoeren van diverse metingen en de grappen bij de dagelijkse visite. Verder ook ben ik jullie veel dank verschuldigd voor de zeer aangename werksfeer die er altijd was op de afdeling. Daarnaast zal ik jullie relatiebemiddeling nooit meer vergeten. Voor alles hartelijk dank. 
Medewerkers laboratorium weefseltypering, in het bijzonder Marian Kik, jullie wil ik bedanken voor alle steun en uitleg bij het afdraaien en bewerken van de bloeden urinemonsters en de opslag in de diverse vriezers.

Robert-Jan van Suylen, jij was van onschatbare waarde in het herbeoordelen en classificeren van de talloze biopten.

Fred Nieman, jou wil ik bedanken voor de uitgebreide variantie-analyse. Zonder jouw medewerking waren we hier nooit uitgekomen.

Pamela Falger, ook al deed ik nog zo mijn best om waardig Engels te schrijven, jij wist het altijd weer te verbeteren en tot een prettig lopende tekst om te toveren. Hiervoor mijn hartelijke dank.

Tiny Wouters, jouw verwerking van teksten tot een toonbaar manuscript en vervolgens proefschrift blijft van onschatbare waarde. Zonder jou had ik dit nooit voor elkaar gekregen en was de uiteindelijke afronding van het proefschrift nooit zo vlot verlopen.

De leden van de beoordelingscommissie: Prof. dr. M.J.A.P. Daemen, als voorzitter, Prof. dr. J. Floege, Dr. T. Goto, Prof. dr. P.J.E.H.M. Kitslaar en Prof. dr. C.D.A. Stehouwer, jullie wil ik bedanken voor de bereidwilligheid om dit proefschrift te doorgronden. Math Daemen, wil ik in het bijzonder bedanken voor zijn snelle reacties, waardoor de afwerking in een stroomversnelling kon komen. Dr. Goto, tacrolimus was developed by you and therefore I am very honoured that you were willing to review my thesis.

Maatschap Interne Geneeskunde, Longziekten en Maag-Darm-Leverziekten van het Medisch Centrum Rijnmond-Zuid, bij jullie heb ik het gevoel gekregen een tweede thuis te hebben gevonden. Verder wil ik jullie bedanken dat jullie mij altijd zijn blijven stimuleren om dit proefschrift af te ronden. In het bijzonder wil ik ook mijn collega-nefrologen, René van den Dorpel, Peter van de Ven, Pieter Kramer en Coby van de Heijden-Buijssen bedanken voor de prettige werksfeer, die er altijd is ondanks onze grote en drukke afdeling en de extra tijd die jullie mij gunden om de laatste loodjes te voltooien. Hopelijk kunnen we de kennis opgedaan uit dit proefschrift ook in ons ziekenhuis in de praktijk gaan brengen.

De Nierstichting, dankzij jullie financiële ondersteuning werd ik in de gelegenheid gesteld om de opleiding nefrologie te volgen en aan dit proefschrift te beginnen. Hiervoor hartelijk dank. 
Alle patiënten wil ik bedanken voor hun bereidwilligheid om geheel belangeloos mee te werken aan de diverse studies.

Arie van Vliet, langzamerhand wordt jij zo'n beetje de tekenaar van de hele familie. Bij deze wil ik je hartelijk danken voor je ontwerp van de kaft.

De paranimfen, André Tinga en Bart Hermans, jullie zijn niet alleen van belang geweest bij het organiseren van alle festiviteiten, maar jullie zijn door de jaren heen altijd een belangrijke steun voor mij geweest. Hierbij wil ik jullie dan ook heel hartelijk danken.

Mijn ouders, jullie wil ik bedanken dat ik altijd de mogelijkheid heb gekregen om mijzelf te ontwikkelen in die richting die ik graag wilde. 


\section{Publicatielijst}




\section{Publicatielijst}

1. Netten PM, Boots JMM, Bredie SJH, Arend JACJ den, Mol MJTM, Thien Th, Hoefnagels WHL and Lutterman JA. An automated computerized method using Finapress for measuring cardiovascular reflexes. Clin Sci 1992;83:157-63.

2. Boots JMM, Burghouts JThM and Jansen JLJ. Unaccountable severe hypercalcemia in a patient treated for hypoparathyroidism with dihydrotachysterol. Neth J Med 1999;54(1):16-20.

3. Hooff JP van, Boots JMM, Duijnhoven EM van and Christiaans MHL. Dosing and management guidelines for renal transplant patients. Transplant Proc 1999;31(S7A):54S-7.

4. Boots JMM, Duijnhoven EM van, Christiaans MHL, Nieman FHM, Suylen RJ van and Hooff JP van. Single center experience with tacrolimus versus cyclosporin-Neoral in renal transplant recipients. Transplant Int 2001;14(6):370-83.

5. Halaby T, Boots $H$, Ven A van der, Beguin $H$, Vermeulen A, Hooff $H$ van and Jacobs J. Phaeohyphomycosis caused by Alternaria infectioria in a renal transplant recipient. J Clin Microbiol 2001;39(5):1952-5.

6. Duijnhoven EM van, Boots JMM, Christiaans MHL, Wolffenbuttel BHR and Hooff JP van. Influence of tacrolimus on glucose metabolism before and after renal transplantation: a prospective study. J Am Soc Nephrol 2001;12(3):583-8.

7. Kooman JP, Christiaans MHL, Boots JMM, Sande FM van der, Leunissen KML and Hooff JP van. A comparison between office and ambulatory blood pressure measurements in renal transplant patients with chronic transplant nephropathy. Am J Kidney Dis 2001;37(6):1170-6.

8. Duijnhoven EM van, Christiaans MHL, Boots JMM, Nieman FHM, Wolffenbuttel BHR and Hooff JP van. Glucose metabolism in the first 3 years after renal transplantation in patients on tacrolimus versus cyclosporin-based immunosuppression. J Am Soc Nephrol 2002;13(1): 213-20.

9. Boots JMM, Duijnhoven EM van, Christiaans MHL, Wolffenbuttel BHR and Hooff JP van. Glucose metabolism in renal transplant recipients on tacrolimus: The effect of steroid withdrawal and tacrolimus trough level reduction. J Am Soc Nephrol 2002;13(1):221-7.

10. Boots JMM, Duijnhoven EM van, Christiaans MHL, Suylen RJ van and Hooff JP van. Early steroid withdrawal in renal transplant recipients with tacrolimus dual therapy: A pilot study. Transplantation 2002;74(12):1703-9.

11. Boots JMM, Duijnhoven EM van, Christiaans MHL, Suylen RJ van and Hooff JP van. Early steroid withdrawal in renal transplant recipients with tacrolimus dual therapy: A pilot study. Transplant Proc 2002;34(5):1698-9.

12. Duijnhoven EM van, Christiaans MHL, Boots JMM and Hooff JP van. Evidence that fasting does not significantly affect trough levels of tacrolimus in stable renal transplant recipients. Transplant Proc 2002;34(5):1723-5.

13. Duijnhoven EM, Christiaans MHL, Boots JMM, Goossens VJ, Undre NA and Hooff JP van. A late episode of post-transplant diabetes mellitus during active hepatitis $\mathrm{C}$ infection in a renal allograft recipient using tacrolimus. Am J Kidney Dis 2002;40(1):195-201.

14. Boots JMM, Ham ECH van den, Christiaans MHL and Hooff JP van. Risk of adrenal insufficiency with steroid maintenance therapy in renal transplantation. Transplant Proc 2002; 34(5):1696-7. 
15. Boots JMM, Christiaans MHL, Undre NA and Hooff JP van. Pre-transplant pharmacokinetics: Does it predict the dose of tacrolimus after renal transplantation. Transplant Proc 2002;34(8): 3171-2.

16. Artz MA, Boots JMM, Ligtenberg G, Roodnat JI, Christiaans MHL, Hené RJ, Blom RJ, Demacker PN and Hilbrands LB. Randomized conversion from cyclosporine to tacrolimus in renal transplant patients: Improved lipid profile and unchanged plasma homocysteine levels. Transplant Proc 2002;34(5):1793-4.

17. Duijnhoven EM van, Boots JMM, Christiaans MHL, Stolk LML, Undre NA and Hooff JP van. Increase in tacrolimus trough levels after steroid withdrawal. Transpl Int 2003;16(10):721-5.

18. Artz MA, Boots JMM, Ligtenberg G, Roodnat JI, Christiaans MHL, Vos PF, Blom HJ, Sweep CGJ, Demacker PNM and Hilbrands LB. Improved cardiovascular risk profile and renal function in renal transplant patients after randomized conversion from cyclosporine to tacrolimus. J Am Soc Nephrol 2003;14(7):1880-8.

19. Duijnhoven EM van, Boots JMM, Christiaans MHL and Hooff JP van. Metabolic aspects of tacrolimus in renal transplantation. Consequences for the choice of an immunosuppressive regimen and for the management of post-transplant diabetes mellitus. Minerva Urol Nefrol 2003;55(1):33-42.

20. Duijnhoven EM van, Christiaans MHL, Boots JMM, Goossens VJ, Undre NA and Hooff JP van. A late episode of posttransplant diabetes mellitus during active hepatitis $\mathrm{C}$ infection in a renal allograft recipients using tacrolimus. Hepatitis Rev J 2003;2:7-9.

21. Artz MA, Boots JMM, Ligtenberg G, Roodnat JI, Christiaans MHL, Vos PF, Moons P, Borm $G$ and Hilbrands LB. Conversion from cyclosporine to tacrolimus improves quality-of-life indices, renal graft function and cardiovascular risk profile. Am J Transplant 2004;4(6):937-45.

22. Boots JMM, Christiaans MHL and Hooff JP van. Effect of immunosuppressive agents on longterm survival of renal transplant recipients: focus on the cardiovascular risk. Drugs 2004;64(18): 2047-73.

23. Boots JMM. Is tacrolimus superior to ciclosporin microemulsion in preventing long-term acute renal transplant rejection? Nat Clin Pract Nephrol 2005;1(1):16-7.

24. Hooff JP van, Gelens MACJ, Boots JMM, Duinhoven EM van, Dackus J and Christiaans MHL. Preservation of renal function and cardiovascular risk factors. Transplant Proc 2006; in press.

25. Hooff JP van, Gelens MACJ, Boots JMM and Christiaans MHL. Steroid withdrawal/avoidance. The Maastricht experience. Transplant Proc 2006; in press. 


\section{Curriculum vitae}




\section{Curriculum vitae}

Johannes (Hans) Martinus Maria Boots werd op 23 mei 1965 geboren in Roermond (Limburg).

Hier voltooide hij in 1983 het gymnasium $\beta$ aan het Bisschoppelijk College Schöndeln. In datzelfde jaar werd gestart met de studie geneeskunde aan de Katholieke Universiteit Nijmegen. In 1987 werd hier het doctoraal examen behaald en op 1 juni 1990 volgde het artsexamen. Tijdens deze studiefase ontving hij een prijs van de Nederlandse Vereniging voor Diabetes Onderzoek voor zijn wetenschappelijke stage: "Een geautomatiseerd testprogramma ter detectie van diabetische autonome neuropathie. Reproduceerbaarheid bij jonge gezonde proefpersonen", begeleider Dr. P.M. Netten.

Direct aansluitend aan zijn artsexamen ging hij als arts-assistent werken op de afdeling Medische Oncologie van het Sint Radboudziekenhuis in Nijmegen (thans Universitair Medisch Centrum Nijmegen). In oktober 1990 begon zijn dienstplicht, waarbij hij werkte als stafarts bij de Provinciaal Geneeskundige Dienst van het district Limburg. Na de dienstperiode werkte hij nog een maand bij de afdeling Medische Oncologie van het Sint Radboudziekenhuis in Nijmegen en vanaf maart 1992 als arts-assistent interne geneeskunde in het Groot Ziekengasthuis (thans Jeroen Bosch Ziekenhuis) in 's Hertogenbosch. Vanaf augustus 1993 werkte hij als arts-assistent interne geneeskunde in het Maasland Ziekenhuis (thans Orbisconcern) in Sittard. Hier werd in oktober 1993 gestart met de opleiding tot internist (opleider Dr. A.M.J. Moers). Deze opleiding werd vanaf april 1995 voortgezet in het Academisch Ziekenhuis Maastricht (opleider Prof. dr. H.F.P. Hillen).

In oktober 1999 volgde de registratie tot internist. Hierna werd gestart met de opleiding tot nefroloog (opleiders Prof. dr. J.P. van Hooff en Prof. dr. K.M.L. Leunissen) en werd gestart met het onderzoek, zoals beschreven in dit proefschrift. In deze periode werkte hij een maand in het Horacio Oduber Hospitaal in Aruba. In april 2001 volgde de registratie als nefroloog. Hierna werkte hij nog een jaar als juniorstaflid op de afdeling nefrologie van het Academisch Ziekenhuis Maastricht. Daarna volgden waarnemingen als internistnefroloog in het Viecurie Medisch Centrum in Venlo en het Orbisconcern in Sittard.

Vanaf juni 2003 is hij werkzaam als internist-nefroloog in het Medisch Centrum Rijnmond-Zuid in Rotterdam, anvankelijk op de locatie Clara en vanaf juni 2005 op de locatie Zuider.

Op 6 september 2002 trouwde hij met Sabine Deferme. Binnenkort verwachten zij hun eerste kind. 